\title{
Cellular immunotherapy of pancreatic ductal adenocarcinoma: Discovery and evaluation of novel target candidates
}

\section{Dissertation}

for the award of the degree

"Doctor rerum naturalium"

of the Georg-August-Universität Göttingen

within the doctoral program Molecular Medicine

of the Georg-August University School of Science (GAUSS)

submitted by

Daniel Schäfer

from Freudenberg, Germany

Göttingen 2020 


\section{Thesis Committee}

Prof. Dr. Frauke Alves

Translationale Molekulare Bildgebung, Max-Planck-Institut für Experimentelle Medizin Klinik für Hämatologie und Medizinische Onkologie, Universitätsmedizin Göttingen Institut für Diagnostische und Interventionelle Radiologie, Universitätsmedizin Göttingen

Prof. Dr. Hubertus Jarry

Klinische und Experimentelle Endokrinologie, Universitätsmedizin Göttingen

Prof. Dr. Ralf Dressel

Institut für Zelluläre \& Molekulare Immunologie, Universitätsmedizin Göttingen

\section{Members of the Examination Board}

Referee: Prof. Dr. Frauke Alves

Translationale Molekulare Bildgebung, Max-Planck-Institut für Experimentelle Medizin Klinik für Hämatologie und Medizinische Onkologie, Universitätsmedizin Göttingen Institut für Diagnostische und Interventionelle Radiologie, Universitätsmedizin Göttingen

$2^{\text {nd }}$ Referee: Prof. Dr. Hubertus Jarry

Klinische und Experimentelle Endokrinologie, Universitätsmedizin Göttingen

$3^{\text {rd }}$ Referee: Prof. Dr. Ralf Dressel

Institut für Zelluläre \& Molekulare Immunologie, Universitätsmedizin Göttingen

\section{Further members of the Examination Board}

Prof. Dr. Heidi Hahn

Institut für Humangenetik, Universitätsmedizin Göttingen

Prof. Dr. Luis Pardo

Onkophysiologie, Max-Planck-Institut für Experimentelle Medizin

\section{Prof. Dr. Lutz Walter}

Institut für Zelluläre \& Molekulare Immunologie, Universitätsmedizin Göttingen

Date of oral examination: 11.01.2021 


\section{Content}

LIST OF FIGURES....................................................................................................................................... 4

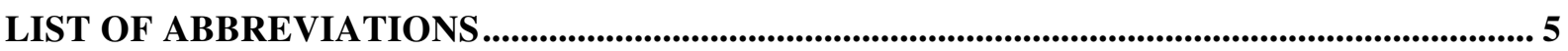

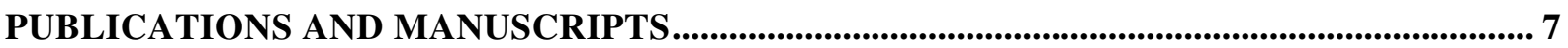

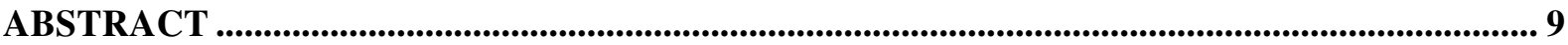

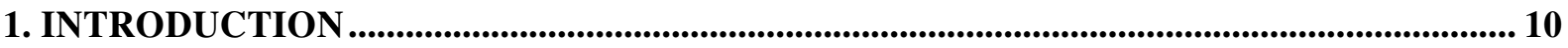

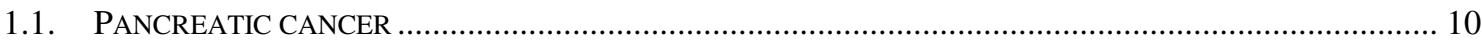

1.2. THE HEALTHY PANCREAS AND PANCREATIC DUCTAL ADENOCARCINOMA DEVELOPMENT ...................... 10

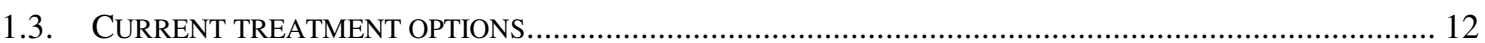

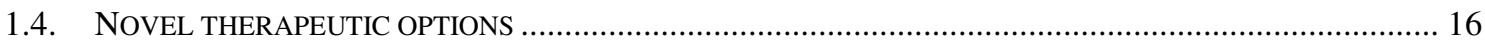

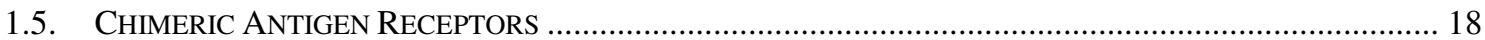

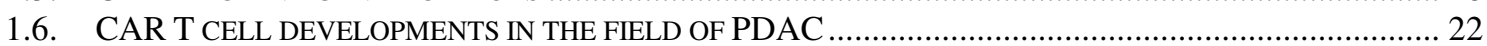

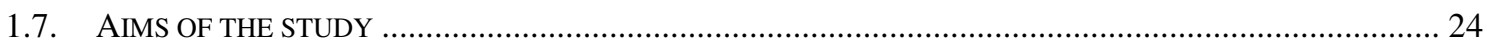

2. INDIVIDUAL CONTRIBUTIONS TO THE MANUSCRIPTS …............................................ 26

CHAPTER I - CD318, TSPAN8 AND CD66C RESEMBLE NOVEL CANDIDATES FOR CAR T CELL BASED IMMUNOTHERAPY OF PANCREATIC ADENOCARCINOMA ………................................................................. 26 CHAPTER II - A NOVEl SigleC-4 DERIVED SPACER IMPROVES THE FunCTIONALITY OF CAR T CELLS

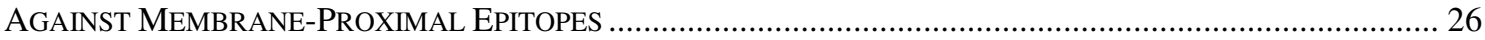

3. CHAPTER I - CD318, TSPAN8 AND CD66C RESEMBLE NOVEL CANDIDATES FOR CAR T CELL BASED IMMUNOTHERAPY OF PANCREATIC ADENOCARCINOMA....... 28

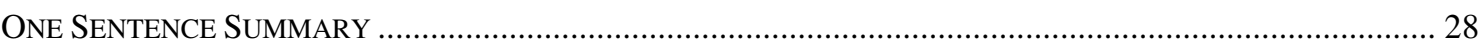

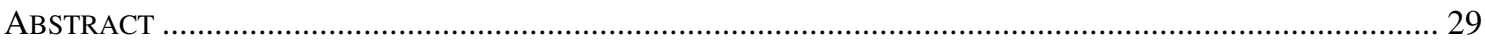

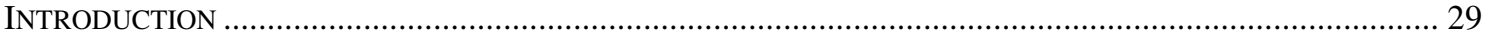

RESULTS ... ……

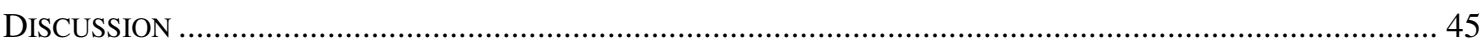

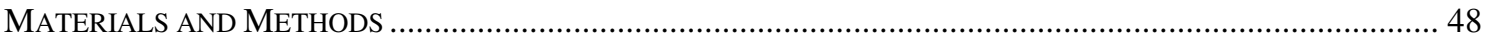

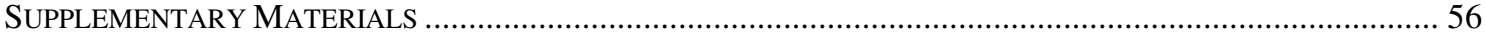

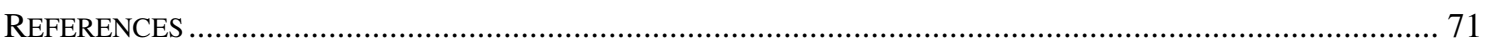

4. CHAPTER 2 - A NOVEL SIGLEC-4 DERIVED SPACER IMPROVES THE

FUNCTIONALITY OF CAR T CELLS AGAINST MEMBRANE-PROXIMAL EPITOPES... 78

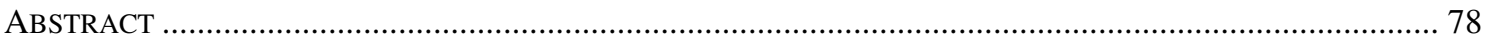

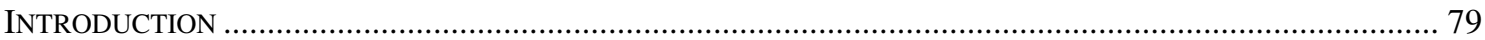

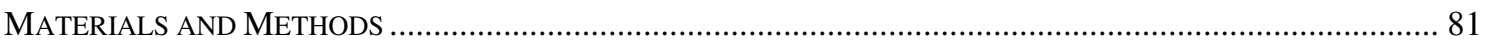

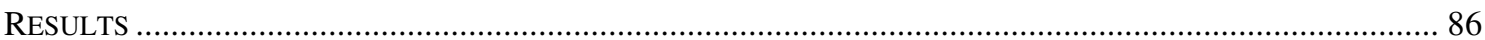

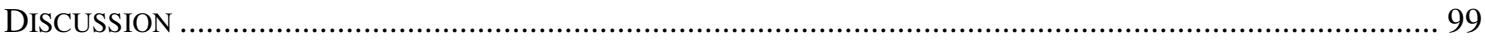

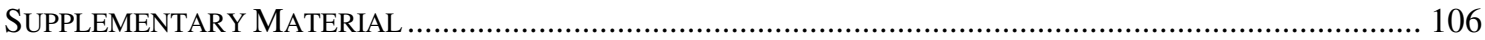

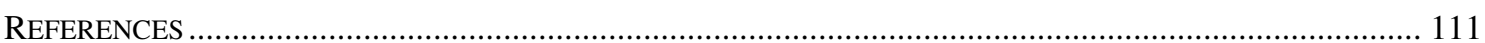

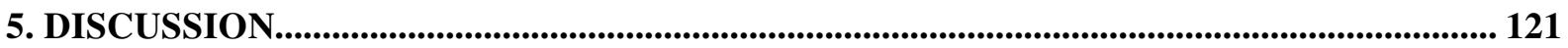

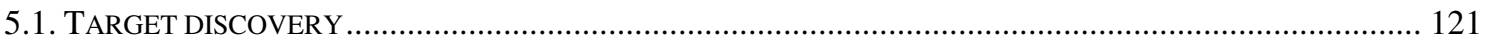

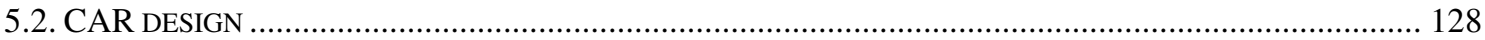

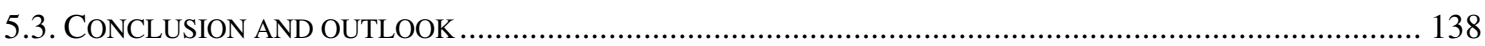

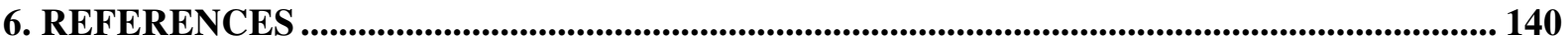

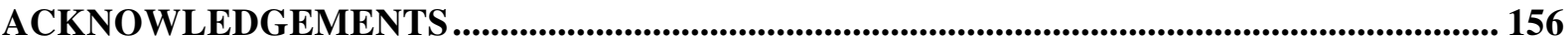

CURRICULUM VITAE ...................................................................................................................... 158 


\section{List of figures}

Figure 1: Proposed process of pancreatic ductal adenocarcinoma (PDAC) development........................ 12

Figure 2: Exemplary treatment algorithm summarizing current treatment options for PDAC................ 16

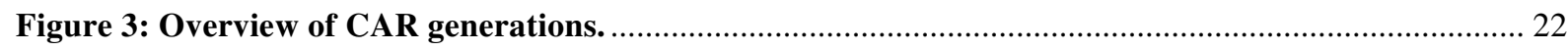

Figure 4: Increasing CAR T cell specificity by applying Boolean AND gates: Part I........................... 129

Figure 5: Increasing CAR T cell specificity by applying Boolean AND gates: Part II. .......................... 130

Figure 6: Increasing CAR T cell specificity by applying a Boolean NOT gate................................... 131

Figure 7: Hypothetical steric CAR:antigen interactions at a CAR synapse......................................... 135 


\section{List of abbreviations}

\begin{tabular}{|c|c|}
\hline $5-\mathrm{FU}$ & 5-fluorouracil \\
\hline $\mathrm{aa} / \mathrm{AA}$ & Amino acid \\
\hline $\mathrm{ADM}$ & Acinar-to-ductal metaplasia \\
\hline AICD & Activation induced cell death \\
\hline BLI & Bioluminescence \\
\hline CAR & Chimeric antigen receptor \\
\hline CD318/CDCP1 & CUB domain-containing protein 1 \\
\hline CD66c/CEACAM6 & Carcinoembryonic antigen-related cell adhesion molecule 6 \\
\hline CEA & Carcinoembryonic antigen \\
\hline CLA & Cutaneous lymphocyte antigen \\
\hline $\mathrm{CO} 1 / 2$ & Co-stimulatory domain $1 / 2$ \\
\hline CRS & Cytokine release syndrome \\
\hline $\mathrm{E}: \mathrm{T}$ & Effector:target \\
\hline ECM & Extracellular matrix \\
\hline ECOG & Eastern Cooperative Oncology Group \\
\hline EGFR & Epidermal growth factor \\
\hline EMA & European Medicines Agency \\
\hline EpCAM & Epithelial cell adhesion molecule \\
\hline $\mathrm{Fc}$ & Crystallizable fragments \\
\hline FcR & Fc-Receptor \\
\hline FDA & Food and Drug Administration \\
\hline FOLFIRINOX & Folinic acid, 5-fluorouracil, irinotecan, oxaliplatin \\
\hline GTEx & Gene tissue expression project \\
\hline $\mathrm{HE}$ & Hematoxylin and eosin \\
\hline HER2 & Human epidermal growth factor receptor 2 \\
\hline HPA & Human Protein Atlas \\
\hline HPM & Human Proteome Map \\
\hline iCAR & Inhibitory CAR \\
\hline ID & Inhibitory domain \\
\hline IF & Immunofluorescence \\
\hline $\mathrm{Ig}$ & Immunoglobulin \\
\hline IL & Interleukin \\
\hline ITAM & Immunoreceptor tyrosine-based activating motif \\
\hline LNGFR & Low-affinity nerve growth factor receptor \\
\hline mFOLFIRINOX & modified FOLFIRINOX \\
\hline MOI & Marker of interest \\
\hline $\mathrm{mOS}$ & Median overall survival \\
\hline MSLN & Mesothelin \\
\hline MUC1 & Mucin-1 \\
\hline NSCLC & Non-small-cell lung cancer \\
\hline PanIN & Pancreatic intra-epithelial neoplasia \\
\hline PARP & Poly ADP ribose polymerase \\
\hline PBMCs & Peripheral blood mononuclear cells \\
\hline PDAC & Pancreatic ductal adenocarcinoma \\
\hline PDB & ProteomicsDB \\
\hline PDX & Patient derived xenograft \\
\hline PROM1 & Prominin-1 \\
\hline PSCA & Prostate stem cell antigen \\
\hline RNA & Ribonucleic acid \\
\hline
\end{tabular}


$\mathrm{ScFV}$

SELPLG

SIGLEC

SLAM

SP

synNotch

$\mathrm{T}_{\mathrm{CM}}$

TCR

$\mathrm{T}_{\mathrm{EM}}$

TF

TM

TME

TSCM

TSPAN8

$\mathrm{V}_{\mathrm{h}}$

$\mathrm{V}_{1}$

VSV-G
Single-chain variable fragment

Selectin P ligand

Sialic acid-binding immunoglobulin-type lectins

Signaling lymphocytic activation molecule

Spacer

Synthetic Notch receptors

Central memory $\mathrm{T}$ cell

T-cell receptor

Effector memory T cell

Transcription factor

Transmembrane domain

Tumor microenvironment

Stem cell memory T cell

Tetraspanin-8

Variable chain heavy

Variable chain light

Vesicular stomatitis virus glycoprotein $\mathrm{G}$ 


\section{Publications and manuscripts}

\section{Peer reviewed publication}

Schäfer, D., Henze, J., Pfeifer, R., Schleicher, A., Brauner, J., Mockel-Tenbrinck, N., Barth, C., Gudert, D., Al Rawashdeh, W., Johnston, I.C.D. and Hardt, O. (2020). A Novel Siglec-4 Derived Spacer Improves the Functionality of CAR T Cells Against Membrane-Proximal Epitopes. Frontiers in Immunology 11(1704). doi: 10.3389/fimmu.2020.01704.

\section{$\underline{\text { Under revision }}$}

Schäfer, D., Tomiuk, S., Küster, L.N., Al Rawashdeh, W., Henze, J., Tischler-Höhle, G., Agorku, D.J., Brauner, J., Linnartz, C., Lock, D., Kaiser, A. Herbel, C., Eckardt, D., Schüler, J., Ströbel, P., Missbach-Güntner, J., Alves, F., Bosio, A. and Hardt, O. (2020) CD318, TSPAN8 and CD66c resemble novel candidates for CAR T cell based immunotherapy of pancreatic adenocarcinoma. (under revision at Nature Communications)

\section{$\underline{\text { Poster presentations }}$}

Schäfer, D., Küster, L.N., Tomiuk, S., Bosio, A., Missbach-Güntner, J., Pinkert-Leetsch, Diana, Alves, F., Hardt, O. (2019) Cellular immunotherapy for pancreatic cancer: Evaluation of novel target candidates. 39. Jahrestagung des Deutschen Pankreas Club e.V., Göttingen Germany

Henze, J., Schäfer, D., Deppenmeier, M., Schröer, U., Bosio, A., Hardt, O., Al Rawashdeh, W. (2019) In vivo labelling for ex vivo 3D tracking of CAR T cells. MoBi 2019, Münster, Germany Herbel, C., Dittmer, V., Martinez-Osuna, m., Küster, L.N., Schäfer, D., Mallmann, P., Mallmann, M., Ratiu, D., Ströbel, P., Drewes, J., Kollet, Müller, w., Hardt, O., Eckardt, D., Bosio, A. (2019) Evaluation of tumor-associated antigen expression with the MACSima ${ }^{\text {TM }}$ Platform enabling high-content imaging. American Association for Cancer Research Annual Meeting 2019, Atlanta, United States of America

\section{Oral Presentations}

Schäfer, D., "Cellular immunotherapy for pancreatic cancer: Evaluation of novel target candidates”. (2018) MoBi 2018, Göttingen, Germany

(continued on next page) 
Schäfer, D., "Communication and presentation skills". (2019) Kick-off Meeting 1st Consortium Meeting Winter School | EC Innovative Training Network MATURE-NK - ETN765104, Bergisch Gladbach, Germany

\section{Patent application}

Schäfer, D., Pfeifer, R., Johnston, I.C.D., EP20177214.2, "Chimeric antigen receptor with a spacer comprising C2-set Ig-like domains", 28.05.2020 


\section{Abstract}

Pancreatic ductal adenocarcinoma (PDAC) is a devastating disease with a poor prognosis and limited treatment options. In recent years, chimeric antigen receptor (CAR) T cells have achieved remarkable success in the treatment of liquid malignancies but efficacy in solid malignancies is limited to date. A major hurdle is the lack of tumor specific targets. We aimed to address this issue in the case of PDAC by analyzing the expression of almost 400 cell-surface antigens on tumor cells of different patient derived xenograft (PDX) mouse models. Identified candidates were prioritized with respect to their off-tumor expression bioinformatically by using RNA and protein expression databases. The specificity of these target candidates was further validated on primary PDAC specimens using flow cytometry and a newly developed cyclic immunofluorescence (cyclic IF) microscopy technology. We identified Cutaneous Lymphocyte Antigen (CLA), CEACAM6 (CD66c), CDCP1 (CD318) and Tetraspanin-8 (TSPAN8) as having high specificity to tumor cells and restricted off-tumor expression. In addition, we investigated the expression of these targets using flow cytometry and cyclic IF microscopy on healthy tissue arrays. Target expression on healthy tissues was very restricted, which further underlined the quality of the novel target candidates. We generated CAR constructs differing in spacer length (XS, S, M, L) and scFv orientation $\left(\mathrm{V}_{\mathrm{h}}-\mathrm{V}_{\mathrm{l}}, \mathrm{V}_{\mathrm{l}}-\mathrm{V}_{\mathrm{h}}\right)$ for each target candidate and thoroughly validated these CARs in co-culture assays that utilized cytotoxicity, activation marker expression and cytokine release as readouts. All CLA CARs were dysfunctional due to self-antigenicity on activated $\mathrm{T}$ cells. The most promising constructs for the other target candidates were evaluated in two pre-clinical PDAC mouse models. This revealed CD318 $\mathrm{V}_{\mathrm{h}}-\mathrm{V}_{1} \mathrm{XS}$, CD66c $\mathrm{V}_{1}-\mathrm{V}_{\mathrm{h}} \mathrm{XS}$ and TSPAN8 $\mathrm{V}_{\mathrm{h}}-\mathrm{V}_{1} \mathrm{~S}$ as the most effective CAR constructs of the remaining targets. During CAR development we observed inferior in vivo functionality of long IgG-based spacers compared to their in vitro performance. It has been shown that these spacers convey unspecific binding in murine models. However, long spacers are regarded as beneficial when targeting membrane proximal epitopes. Thus, we evaluated a novel class of long spacers, derived from the SIGLEC family, with similar structural attributes as the IgG family but lack the natural unspecific binding sites. In non-solid and solid tumor settings, a novel SIGLEC-4 based spacer demonstrated superior functionality over long IgG spacers in vitro and in vivo with favorable activation marker expression and cytokine release for putative subsequent clinical applications. In summary, this project led to the identification of novel target candidates for PDAC and establishment of a set of highly functional targetspecific CARs that may pave the road for later clinical application and hopefully will help PDAC patients by establishing more efficient treatment options in the future. 


\section{Introduction}

\subsection{Pancreatic cancer}

Pancreatic cancer is the fourth most common cause of cancer associated death in Western countries (Ferlay et al., 2018). Improvements in the treatment of other cancer entities as well as an aging society is supposed to cause pancreatic cancer becoming the second leading cause of cancer related mortality by 2030 (Rahib et al., 2014). The five-year overall survival rate has improved from $5 \%$ to $8 \%$ in the last decade; however, this encouraging trend is not based on the development of new therapeutic approaches (Siegel et al., 2012; Siegel et al., 2018; Lai et al., 2019). Chemotherapy remains the main pillar of pancreatic cancer treatment (Kleeff et al., 2016). Until today, surgery is the only potential curative intervention and has evolved from a high-risk procedure to a difficult but manageable procedure in specialized centers (Hartwig et al., 2013). However, only $20 \%$ of the patients present a resectable tumor at the timepoint of diagnosis, mainly because the remaining cases have already entered a non-resectable or metastatic stage, which frequently occurs due to lack of disease specific symptoms (Gillen et al., 2010; Werner et al., 2013). Genetic predisposition and lifestyle related factors influencing development of pancreatic cancer are known, yet they explain only a minor fraction of the actual incidence number. A familial history of pancreatic cancer can be found in $10 \%$ of the patients (Turati et al., 2013). Genetic mutations most commonly affect the genes BRCA2, BRCA1, PALB2, CDKN2A, ATM, TP53, MLH1, MSH2, MSH6 (Pihlak et al., 2017). Smoking (Parkin, 2011; Bosetti et al., 2012; Whiteman et al., 2015), obesity, low activity (Behrens et al., 2015; Genkinger et al., 2015), nutritional factors (Larsson and Wolk, 2012; Bosetti et al., 2013; Rohrmann et al., 2013) and diabetes (Bosetti et al., 2014) are linked to increased lifetime risks. However, the field is being far away from a comprehensive understanding of the biology of pancreatic cancer, and with therapeutic advancements failing to keep pace with the rising incidence, there is an urgent need for novel therapeutic options.

\subsection{The healthy pancreas and pancreatic ductal adenocarcinoma development}

The healthy pancreas consists of an exocrine and an endocrine compartment, of which the former is producing digestive enzymes and the latter one regulates pancreatic hormone secretion. The endocrine cells steer glucose homeostasis by secreting hormones into the bloodstream. They arrange in clusters called Langerhans islets and comprise $\alpha-, \beta-, \delta-, \varepsilon^{-}$and PP-cells. The exocrine compartment consists of acinar cells secreting zymogens that are locally neutralized by bicarbonate producing ductal epithelial cells and transported to the duodenum 
(Cleveland et al., 2012). Situated in between these two cell types are the centro-acinar cells, which are suggested to act as stem cell-like progenitors for endocrine and exocrine fates (Rovira et al., 2010; Kopinke et al., 2012). From each of these cell types malignancies may arise. However, pancreatic ductal adenocarcinoma (PDAC) is by far the most common neoplasm of the pancreas. It emanates from the aforementioned ductal epithelial or ductal-like cells and accounts for about $80 \%$ of all tumors of the pancreas (Kleeff et al., 2016).

The development of PDAC is likely to start with acinar-to-ductal metaplasia (ADM) (Kanda et al., 2012; Sethi et al., 2017). Initiated by environmental stress conditions, such as inflammation or damaged tissue, acinar cells transdifferentiate to phenotypically epithelial cells (Kopp et al., 2012; Wang et al., 2019). This requires the reprogramming of the cells to stem cell-like fates, rendering them more susceptible towards mutations due to increased proliferation. A key mutation towards PDAC development is the activation of the GTPase KRAS (Fig. 1). 95\% of carcinomas derived from the exocrine pancreas compartment contain KRAS mutations (Almoguera et al., 1988). Studies conducted with transgenic mice bearing KRAS activating mutations showed formation of PDAC precursor lesions and periductal inflammation, but did not develop PDAC (Brembeck et al., 2003; Grippo et al., 2003). These precursor lesions, pancreatic intra-epithelial neoplasia (PanINs), are commonly regarded as evolutionary forerunners of PDAC (Makohon-Moore et al., 2018). These findings underline the importance of KRAS mutations in the oncogenesis of PDAC but show at same time that KRAS activation alone is not sufficient for PDAC establishment. Several more mutations must be accumulated during different PanIN stages, increasing the aggressiveness of cell growth until invasive PDAC is formed. In the last years, a strong body of genome sequencing studies has elucidated that besides KRAS, genetic alterations can be found in the tumor suppressor genes TP53, SMAD4 and $C D K N 2 A$ in around $50 \%$ to $80 \%$ of PDAC patients (Waddell et al., 2015; Witkiewicz et al., 2015; Bailey et al., 2016). Other mutations are highly heterogeneous among patients but also within a single patient at different lesion sides (Witkiewicz et al., 2015; Cancer Genome Atlas Research Network. Electronic address and Cancer Genome Atlas Research, 2017). 


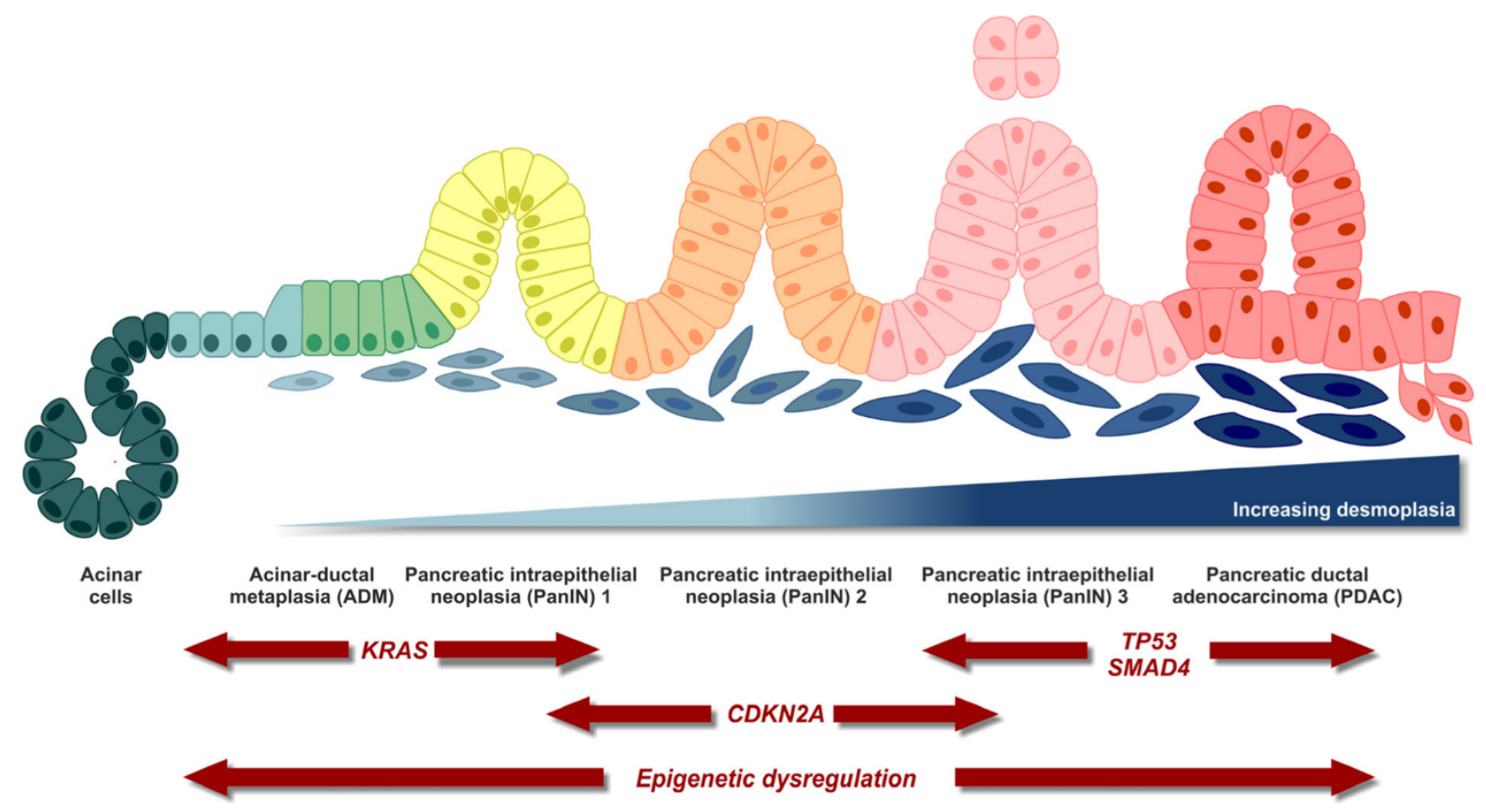

Figure 1: Proposed process of pancreatic ductal adenocarcinoma (PDAC) development. PDAC has its origin either in ductal cells or in acinar cells that underwent acinar-to-ductal metaplasia (ADM) beforehand. Activating mutations of the KRAS gene are found in $>90 \%$ of PDACs (Almoguera et al., 1988). The KRAS mutation is sufficient for induction of precursor lesions called pancreatic intraepithelial neoplasia (PanIN) (Brembeck et al., 2003). Over time, mutations of CDKN2A, TP53 and SMAD4 accumulate leading to a higher degree of desmoplasia through the different PanIN stages. Aggressiveness increases until PDAC is formed. Modified from (Orth et al., 2019).

\subsection{Current treatment options}

At the time of diagnosis, patients are stratified according to the tumor stage and patient performance status (Neoptolemos et al., 2018). Tumor stages are categorized as: 1.) Resectable, 2.) Borderline-resectable/Locally advanced unresectable and 3.) Metastatic. Patient status is normally evaluated based on the Eastern Cooperative Oncology Group (ECOG) score (Oken et al., 1982). The ECOG score ranges between 0 and 5 . While 0 means the patient is asymptomatic, death is categorized as 5. ECOG score of 1 means the patient is symptomatic but not heavily restricted in life style. ECOG 2 indicates that the patient lies less than $50 \%$ of the time in bed but is not able to carry out work activities. A score of 3 means a confinement of more than $50 \%$ of the time to a bed or chair, while a score of 4 indicates the patient is only bedbound. PDAC is mainly asymptomatic during early stages of development, which often leads to its late detection. This has unfortunate implications for the patients, as outcomes and treatment efficacy are more efficient at early stages when the tumor is operable. When the carcinoma is borderline resectable, the primary aim is decreasing the tumor's critical mass to enable surgery. If the tumor is irresectable or metastatic, the only option so far is to offer the best palliative care. 


\subsubsection{Surgery}

Surgery remains the only potential curative therapeutic option. However, diagnosis is often made when the tumor already has vascular involvement or spread to neighboring organs (30$40 \%$ of cases), or distant metastases are present (50-60\% of cases) (Gillen et al., 2010; Werner et al., 2013) rendering a surgery obsolete. Until very recently, surgery was frequently associated with unbearable morbidity and mortality (Bramhall et al., 1995), with unclear benefit on overall survival (Gudjonsson, 1995; Carpelan-Holmström et al., 2005). Therefore, tumor excisions were neglected as valid treatment options for a long time (Bilimoria et al., 2007). This changed with the emergence of more specialized centers and better perioperative handling, decreasing surgery-associated mortality rates below 5\% (Hartwig et al., 2011). A complete pancreatectomy is avoided where possible because it leaves the patient with diabetes (Hartwig et al., 2015). Tumors located in the pancreatic tail are excised through a distal pancreatectomy and splenectomy, while neoplasms of the head are removed by a partial pancreaticoduodenectomy, also referred to as "Whipple procedure" (Diener et al., 2014). Although surgery offers the best chances for a curative setting, $90 \%$ of the patients relapse postsurgery without any additional therapy (Griffin et al., 1990). Thus, adjuvant therapies have become standard-of-care follow-up procedures after tumor resection (Neoptolemos et al., 2004; Neoptolemos et al., 2010; Oettle et al., 2013).

\subsubsection{Adjuvant therapy}

A study that paved the way for usage of 5-fluorouracil (5-FU) in pancreatic cancer until today was conducted in the mid-1970s. The Gastrointestinal Tumor Study Group compared 5-FU and radiation as adjuvant therapy with a non-adjuvant control group. The median survival of the treatment group exceeded the control group's median overall survival (mOS) by 9 months (20 months versus 11 months) (Kalser and Ellenberg, 1985). Notably, although commonly applied in the adjuvant setting, radiation therapy provided no additional benefits over chemotherapy alone (Neoptolemos et al., 2004). In 2008 the CONKO-001 trial introduced gemcitabine as a second regimen for adjuvant chemotherapy (Neuhaus et al., 2008). Consequently, in 2010 the ESPAC-3 trial compared gemcitabine and 5-FU therapy and found no superiority of either one with respect to overall survival (Neoptolemos et al., 2010). Both groups exhibited a mOS of 23 months. Later trials focused more on chemotherapeutics combining several agents. A breakthrough was achieved during the JASPAC-1 investigation in 2016, in which gemcitabine was compared to S-1, a combination of tegafur, gimeracil and oteracil. The S1 treated arm reached an unprecedented mOS of 46.5 months, while the gemcitabine monotherapy arm 
reached 25.5 months (Uesaka et al., 2016). Although promising, these results were not yet confirmed in non-Asian populations. The current standard of care is FOLFIRINOX, either in the original formulation or in a modified version (mFOLFIRINOX) to reduce side effects. FOLFIRINOX consists of 5-FU, folinic acid, irinotecan and oxaliplatin. In the GI PRODIGE 24 trial, published in 2018, the patient cohort post-operatively treated with FOLFIRINOX reached a mOS of 54.4 months. The gemcitabine monotherapy cohort reached a mOS of 35 months (Conroy et al., 2018). Of note, the number of adverse events was higher within patients of the combination therapy cohort, most likely caused by the higher cytotoxic potential of FOLFIRINOX. Thus, it is advised to administer either gemcitabine monotherapy or gemcitabine/capecitabine dual therapy to patients with a bad performance status as shown in the ESPAC-4 trial (Neoptolemos et al., 2017).

\subsubsection{Neoadjuvant therapy}

Neoadjuvant therapy aims at preconditioning the tumor in order to allow for subsequent surgery. This is especially important for the $30-40 \%$ of the patients who present with a "borderline-resectable/locally advanced" tumor. These tumors have a lower resection rate due to vascular and surrounding organ involvement of the tumor. Neoadjuvant therapy can eliminate micro-metastases and decrease the tumor size (Seufferlein and Ettrich, 2019), which may potentially transform borderline-resectable tumors into resectable ones. Currently, there is no consensus about the most suitable treatment protocols, pre- and post-operative, which is in part due to missing high-quality data trials (Neoptolemos et al., 2018). While pre-operative chemo- or chemoradiotherapy with gemcitabine alone or in combination with oxaliplatin (Heinrich et al., 2011; Tachezy et al., 2014; Versteijne et al., 2016) was investigated in the past, the rise of (m)FOLFIRINOX led to a shift from chemoradiotherapy to sole chemotherapy as pre-operative treatment (Katz et al., 2016). Since FOLFIRINOX contains 5-FU, gemcitabine is preferred in the subsequent adjuvant therapy. The results of the ESPAC-5F trial (ISRCTN89500674) comparing upfront surgery, chemoradiotherapy, gemcitabinecapecitabine and FOLFIRINOX are expected by the end of 2020 and could clarify which strategy is most promising. It should be mentioned that also in the neoadjuvant setting, only well performing patients qualify for FOLFIRINOX based therapy.

\subsubsection{Palliative therapy}

The only treatment option for patients with distant metastases or locally advanced irresectable tumors is palliative care. For decades, 5-FU remained the only available chemotherapeutic agent in the metastatic setting. This changed in 1997, when 5-FU was compared with 
gemcitabine (Burris et al., 1997). Although the survival extension was minimal (mOS gemcitabine 5.65 months vs. 5-FU 4.41 months), gemcitabine had less severe side effects. In 2011 FOLFIRINOX proved to be superior over gemcitabine in the ACCORD-11 trial. FOLFIRINOX treated patients reached mOS of 11.1 months, while gemcitabine treated cohort reached only 6.8 months (Conroy et al., 2011). In the same year as the ACCORD-11 trial the MPACT trial compared the combination of gemcitabine/nab-paclitaxl versus gemcitabine monotherapy. The combination therapy elevated the mOS to 8.7 months from 6.6 months in the monotherapy group (Von Hoff et al., 2011).

\subsubsection{Second line treatment}

Even with the recent advances in surgery, adjuvant and neoadjuvant therapy, most PDAC patients relapse. Around half the patients of the PRODIGE-4 trial later underwent second-line chemotherapy (Conroy et al., 2011). It has been shown that after initial gemcitabine therapy, the mixture of oxaliplatin, 5-FU and folinic acid offers a mOS of 5.9 months, while 5-FU and folinic acid alone reach a mOS of 3.3 months (Oettle et al., 2014). A 2016 published study showed that the combination therapy of nal-irinotecan, 5-FU and folinic acid offers similar mOS (6.1 months) as seen before with oxaliplatin, 5-FU and folinic acid (Wang-Gillam et al., 2016). Phase III studies investigating second line treatment after initial 5-FU containing therapy are missing. Thus, it is currently advised to administer oxaliplatin or nal-irinotecan in combination with 5-FU and folinic acid after initial gemcitabine treatment and nab-paclitaxl with gemcitabine after initial 5-FU usage (Sohal et al., 2016; Sohal et al., 2020).

An exemplary treatment algorithm summarizing current treatment options with expected outcomes, can be found in Figure 2. 


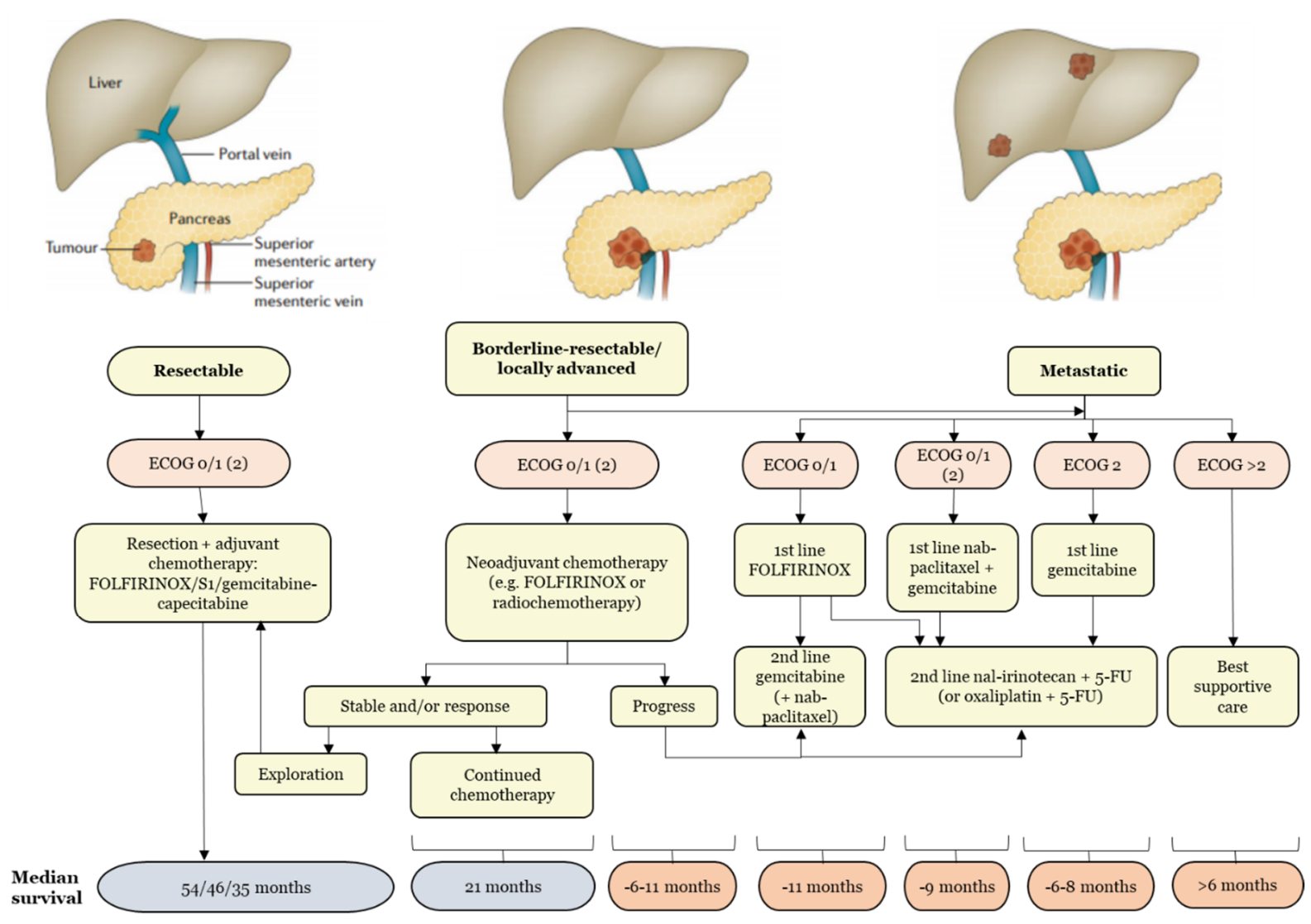

Figure 2: Exemplary treatment algorithm summarizing current treatment options for PDAC. Patients are stratified according to the stage of the tumor (resectable/borderline resectable/metastatic) and patient performance status (Eastern Cooperative Oncology Group (ECOG) score). Median survival in the blue fields has been added from the latest clinical data. Median survival in the red fields refers to first-line survival and is estimated from Neoptolemos et al.. Modified from (Neoptolemos et al., 2018).

\subsection{Novel therapeutic options}

Although regimens developed in recent years can markedly elevate overall survival, especially for resectable tumors, this comes at the cost of increased severe side effects. In addition, the 5year overall survival rate remains low at around $8-9 \%$. Other treatment options are limited to small subpopulations of PDAC patients, and large phase III results are missing. Furthermore, current therapies for metastatic or relapsed patients barely increase the overall survival. This section discusses some of the novel therapeutic options that are currently being explored in the hope of significantly improving treatment options for PDAC patients.

\subsubsection{Targetability of patient specific mutations}

Although the mutational landscape of PDAC consists of only four main genes, very few of these genetic alterations offer druggable targets. This is different for the specific case of the $\mathrm{KRAS}^{\mathrm{G} 12 \mathrm{C}}$ mutation. It is found in $1.5 \%$ of PDAC patients and can be targeted with the small molecule KRAS inhibitor AMG 510 (Canon et al., 2019). Its use showed high efficacy in 
murine xenograft models and also in a preliminary clinical evaluation of KRAS ${ }^{\mathrm{G} 12 \mathrm{C}}$ bearing non-small-cell lung cancer (NSCLC) patients. All patients demonstrated either stable disease or a partial response (Canon et al., 2019). However, these are only initial findings and more data need to be collected, as well as the assessment of its benefit in PDAC. Around 5\% of PDAC patients carry loss-of-function mutations in the genes BRCA1 and BRCA2 (Holter et al., 2015). These genes are involved in homologous recombination repair, which is why this genetic anomaly proved to be sensitive towards inhibition of the enzyme poly ADP ribose polymerase (PARP) with the small molecule olaparib (Golan et al., 2019). PARP is indispensable for single-strand DNA repair and inhibition is therefore inducing apoptosis of the malignant cells. In a recent phase III trial, significantly increased progression-free survival was observed in a patient cohort that was previously sensitive to the DNA damage agent cisplatin (Golan et al., 2019). Nevertheless, the aforementioned therapies are still in an experimental phase, only showed elongation of progression free survival, and - most importantly - are only suitable for selected subpopulations of PDAC patients.

\subsubsection{Immunotherapy}

A new hope for patients with currently incurable neoplastic malignancies comes from immunotherapies. Immunotherapy is a relatively new field within oncological research. It gathered a lot of momentum in the last decade and peaked in the awarding of the 2018 Nobel Prize in Physiology or Medicine to James P. Allison and Tasuku Honjo for discovering a cancer therapy using immune-checkpoint regulation (Guo, 2018). Since the revelation of the pivotal roles of PD-1 and CTLA-4 (Ishida et al., 1992; Leach et al., 1996), blocking their function by antibodies has become a standard-of-care therapy for multiple indications, first and foremost in melanoma (Hodi et al., 2010; Gong et al., 2018). Immune checkpoint inhibition has also been investigated in the setting of pancreatic cancer but could not proof any meaningful benefit (Henriksen et al., 2019). A few reasons have been proposed for this. The development of PDAC comes along with a strong desmoplastic reaction of the tumor microenvironment (TME) (Henze et al., 2020). The TME of PDAC is very hostile towards T cells, the main profiteers of immunecheckpoint inhibition. There are physical barriers, such as a hypovascularized stroma, preventing $\mathrm{T}$ cells from extravasating blood vessels as well as a dense extracellular matrix (ECM), averting effective migration (Henze et al., 2020; Hosein and Brekken, 2020). The stroma is nutrient poor and hypoxic. Even if a T cell reaches a tumor cell, it needs to recognize the tumor cell as malignant, which works via the presentation of mutated neoantigens through the MHC complex and their recognition by the $\mathrm{T}$ cell receptor. It has been shown that tumor 
cells can downregulate MHC molecules and PDAC commonly shows a low mutational burden leading to a very limited number of neoantigens (Chen et al., 2020; Yamamoto et al., 2020). In addition, the TME is filled with immunosuppressive cells, such as regulatory $\mathrm{T}$ cells and myeloid-derived suppressor cells, preventing $\mathrm{T}$ cells from switching to an activated state (Hosein and Brekken, 2020). One approach to overcome these hurdles is the application of chimeric antigen receptor (CAR) engineered $\mathrm{T}$ cells. Thereby, the $\mathrm{T}$ cells are modified to act independent of the MHC/TCR pathway, can be engineered to excrete ECM digestive enzymes (Caruana et al., 2015), can be altered to convert suppressive TME signaling into an activating one and most important (Leen et al., 2014; Mohammed et al., 2017), are a "living drug" making them more persistent and active than small molecules. However, so far CAR T cells proved efficacy mostly in hematologic tumors such as B cell malignancies (Schmidts and Maus, 2018). The reasons for this are thought to be based on two major characteristics: 1 . The tumor cells are located in organs well accessible for the CAR T cells like blood and bone marrow and 2. Tumor targets such as CD19 or CD20 are widely expressed on the tumor cells and harbor very limited on-target off-tumor toxicities (Tokarew et al., 2019).

\subsection{Chimeric Antigen Receptors}

CARs render $\mathrm{T}$ cell activation independent of the MHC/TCR signaling axis by providing a direct link between binding to a cell surface molecule on the target cell and inducing TCR-like signaling. Their modular blueprint consists of four major building blocks: In the extracellular compartment, a CAR comprises an antigen binding domain and a spacer (also referred to as hinge) domain. The spacer domain is followed by a membrane-spanning transmembrane domain and one or more intracellular signal transduction domains (Miliotou and Papadopoulou, 2018).

\subsubsection{CAR design: Antigen binding domain}

Grafting new specificity onto a T cell was pioneered in 1987 by Kuwana et al.. They engineered a new immunoglobulin-derived variable region onto T-cell receptor constant regions (Kuwana et al., 1987). Two years later, this method was refined by Gross et al., who were the first to fuse antibody derived binding domains onto the T-cell receptor (Fig. 3)(Gross et al., 1989). Although, this modified TCR was able to stimulate the T cell, this approach had some insufficiencies. Cloning was laborious and surface expression inefficient. Ultimately, the whole CD3:TCR complex had to form properly to convey activation. This led to the development of a new single polypeptide chain format (Fig.3), incorporating a single-chain variable fragment $(\mathrm{scFv})$ as antigen binding domain (Eshhar et al., 1993). The use of antibody derived binding 
domains also has practical implications: They offer high affinity binding and target candidates, which have been discovered using antibodies, can be targeted with an scFv derived from the very same antibody, streamlining the subsequent CAR design. Thus, most of the antigen binding domains in use today have been derived from the variable heavy $\left(\mathrm{V}_{\mathrm{h}}\right)$ and variable light $\left(\mathrm{V}_{1}\right)$ chains of monoclonal antibodies connected with a flexible linker (Gacerez et al., 2016). The $\mathrm{scFv}$ brings along a set of properties influencing CAR behavior beyond sole antigen recognition. The affinity of an $\mathrm{scFv}$ needs to surpass a certain threshold to be able to activate the $\mathrm{T}$ cell upon antigen binding, but too high affinity can lead to activation induced cell death (AICD)(Watanabe et al., 2014; Lynn et al., 2016; Dwivedi et al., 2018). Also long-term persistence seems to be improved when using low to medium affinity scFvs (Ghorashian et al., 2019). Interestingly, affinity fine-tuning can also be utilized for engineering CARs to spare normal tissues but preserve antitumor activity by inducing $\mathrm{T}$ cell activation only in case of a high level of target expression on the cell surface (Caruso et al., 2015).

\subsubsection{CAR design: Spacer domain}

The antigen binding domain is followed by a spacer domain, sometimes also referred to as hinge (Cartellieri et al., 2010). Originally, the spacer domain was thought as a structural element, which would help the antigen binding domain to reach over the dense glycocalyx of $\mathrm{T}$ cells (Moritz and Groner, 1995). To this end, a variation of different spacer domains have been designed, mainly consisting of immunoglobulin-like domains $(\mathrm{Ig})$ crystallizable fragments $(\mathrm{Fc})$ from antibodies (Hombach et al., 1998; Weijtens et al., 1998; Cooper et al., 2003). Later, domains derived from $\mathrm{CD} 8 \alpha, \mathrm{CD} 28, \mathrm{NKG} 2 \mathrm{D}, \mathrm{TCR} \beta, \operatorname{IgD}, \mathrm{IgG} 1$ and IgG4 have been added (Darcy et al., 1998; Eshhar et al., 2001; Niederman et al., 2002; Zhang et al., 2005; Morgenroth et al., 2007; Barber et al., 2008; Wilkie et al., 2008; Hombach et al., 2010; Jonnalagadda et al., 2015). Notably, comparative studies between spacers and especially among distinct spacer classes are rare in the current literature. An important study providing insight on the influence of CAR spacers was conducted by Patel and colleagues (Patel et al., 1999). They proved that differing spacers in otherwise identical CAR designs lead to differences in CAR stability, cytokine secretion and cytotoxic activity. Later, studies elaborated that CAR T cell activity was not only bound to attributes of the original molecule, but also depended on the position of the respective target epitope. Long CAR spacers evolve higher efficiencies when targeted towards membrane proximal epitopes. On the other hand, short spacers proved higher functionality in the context of membrane distal epitopes (Guest et al., 2005; James et al., 2008; Haso et al., 2013; Hudecek et al., 2013; Krenciute et al., 2016). Thus, the choice of the optimal spacer 
domain needs to be considered wisely. Another point to consider when choosing a spacer domain is the different behavior of some spacers in vitro and in vivo. Some spacers were found to exhibit unspecific Fc-Receptor (FcR) binding capacity in murine models, leading to AICD and their accumulation in the lung (Hombach et al., 2010; Hudecek et al., 2015; Jonnalagadda et al., 2015).

\subsubsection{CAR design: Transmembrane domain}

The transmembrane domain anchors the CAR to the cell surface and connects the extracellular domains to the intracellular domains. Traditionally, this domain is based on peptide sequences from $\mathrm{CD} 3 \zeta, \mathrm{CD} 28$ or $\mathrm{CD} 8 \alpha$. Despite early research revealing the importance of the $\mathrm{CD} 3 \zeta$ transmembrane domain for dimerization with the natural CD3 $\zeta$ frame work and improvement of $\mathrm{T}$ cell activation (Romeo et al., 1992), research into its importance in the overall CAR functionality was long neglected. 18 years later, Bridgeman et al. showed that the optimal antigen response of $\mathrm{CD} 3 \zeta$-based CARs depends on dimerization with the endogenous $\mathrm{CD} 3$ complex (Bridgeman et al., 2010). Not only can the transmembrane domain facilitate dimerization but also influences the molecule's stability. It has been reported that CD3 $\zeta$ incorporating CARs are less stable than CD28 transmembrane harboring CARs (Dotti et al., 2014). In the case of an ICOS-based transmembrane domain, it was found that this domains influences the in vivo phenotype of T cells (Guedan et al., 2018). Also cytokine release is dependent on membrane spanning CAR configuration (Hudecek et al., 2015). Hence, more investigations are needed to better predict CAR functionality in dependence of the transmembrane domain.

\subsubsection{CAR design: Intracellular domain}

The signaling region in the initial CAR constructs was only comprised of the CD3 3 cytoplasmic domain harboring three immunoreceptor tyrosine-based activating motifs (ITAMs). These motifs are phosphorylated in response to antigen binding, which represents the first step in the subsequent signaling cascade resulting in T cell activation (Bezbradica and Medzhitov, 2012). CARs comprising only the $\mathrm{CD} 3 \zeta$ signaling domain are known as first-generation CARs. Albeit

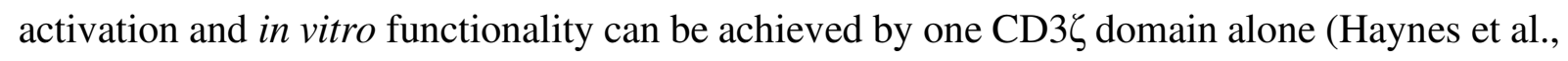
2001), the anti-tumor response of first generation CARs was very limited (Brocker and Karjalainen, 1998; Brocker, 2000; Brentjens et al., 2007). Later it became apparent that T cells require a second - co-stimulatory - signal for full functionality (Fig. 3). One example is CD28 being co-stimulated by B7 presenting cells (Lenschow et al., 1996). As it cannot be expected to find B7 on tumor cells as it is a molecule commonly found on antigen presenting cells, the next 
step was to incorporate the co-stimulus within the CAR framework. Once included, IL-2 production and cell expansion is superior over first-generation CARs (Finney et al., 1998; Maher et al., 2002). Another finding of both studies influencing CAR design until today was the importance of the orientation and positioning of both stimulating domains. Only a membrane proximal co-stimulus and membrane distal $\mathrm{CD} 3 \zeta$ configuration is able to convey a sufficient stimulus. Nowadays, most intensively investigated co-stimulatory domains are either derived from 4-1BB or CD28 (Gacerez et al., 2016). A myriad of other co-stimulating domains has been investigated, including OX40, ICOS, CD27, KIR2DS2, MYD88 and CD40 (Hombach et al., 2012; Song and Powell, 2012; Duong et al., 2013; Hombach et al., 2013; Guedan et al., 2014; Wang et al., 2015; Mata et al., 2017). It was elucidated that CD28 co-stimulus drives CAR T cells into an effector memory ( $\mathrm{T}_{\mathrm{EM}}$ ) phenotype using aerobic glycolysis, while 4-1BB containing CARs differentiate into central memory $\mathrm{T}$ cells $\left(\mathrm{T}_{\mathrm{CM}}\right)$ and rather use oxidative metabolism (Kawalekar et al., 2016). The influence of other co-stimulating domains for T cell activation, differentiation and persistence continues to be a field of active research.

In 2007, CARs of the third generation were introduced (Fig. 3). In the pursuit of better efficacy, Wang et al. combined CD28 and 4-1BB co-stimulatory domains resulting in higher cytotoxicity, cytokine release and proliferation (Wang et al., 2007). In the following years, CAR $\mathrm{T}$ cells proved to be extremely effective in certain settings, with response rates in leukemia from $57 \%-93 \%$ and in lymphoma from $25 \%$ - 100\%, not uncommonly as a last line therapy (June and Sadelain, 2018). The logical consequence was the first approval of commercial CAR T cell products for B cell malignancies (Zheng et al., 2018). This story of success led to the expansion of CAR T cell research targeting other tumor entities. As the range of applications increased, so too did the demands on CAR T cell therapies. In response to the multifactorial hurdles CAR $\mathrm{T}$ cells are facing for the different fields of application, CARs of the fourth and fifth generation have been developed (Fig. 3)(Tokarew et al., 2019). Both generations are based on second generation backbones. Fourth generation CARs constitutively or inducibly produce cytokines, such as IL-12, IL-15 and IL-18 (Hoyos et al., 2010; Pegram et al., 2012; Chmielewski and Abken, 2017). CARs of the fifth generation contain an IL-2 receptor $\beta$-chain, which comprises a binding moiety for STAT3. This leads to a triple pathway activation: 1.) TCR signaling is triggered by $\mathrm{CD} 3 \zeta$, 2.) co-stimulation by $\mathrm{CD} 28$ and 3.) cytokine signaling via the JAKSTAT3/5 pathway (Kagoya et al., 2018). However, future trials have to prove their superiority in a clinical setting. 


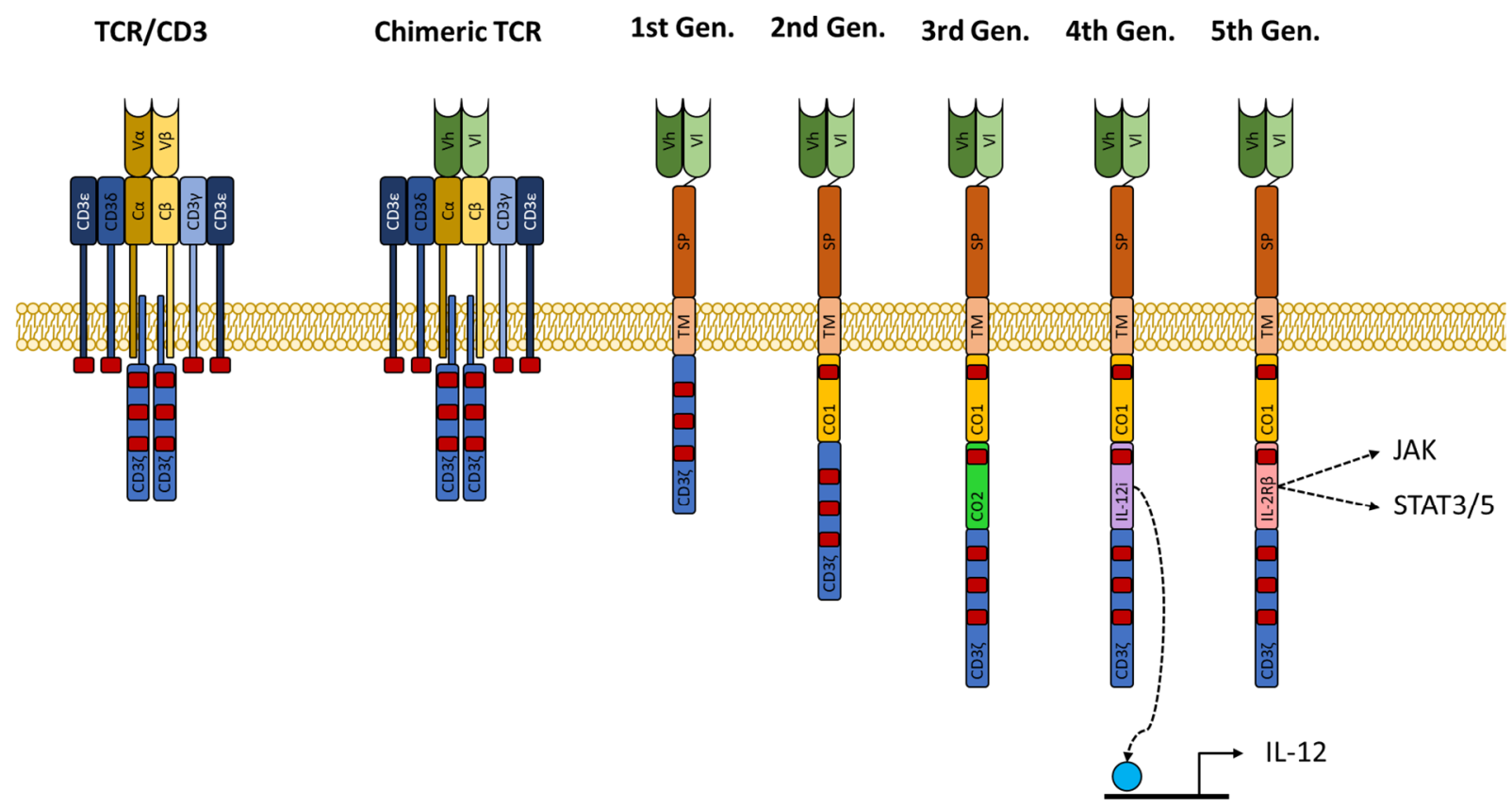

Figure 3: Overview of CAR generations. First attempts to graft novel specificity onto T cells exchanged variable regions of the TCR alpha and beta chains with novel binding moieties (here variable chains from antibodies). From the first generation onwards, CARs consisted of only one polypeptide chain. Binding is usually conveyed by antibody derived single chain variable fragments (scFvs). A spacer (SP) connects the binding moiety with the transmembrane domain (TM). The intracellular domains differ among the generations. First generation CARs comprised only a signaling domain from $\mathrm{CD} 3 \zeta$ containing three immunoreceptor tyrosine-based activating motifs (ITAMs/red boxes). These motifs are phosphorylated in response to antigen recognition, starting the subsequent signaling cascade for $\mathrm{T}$ cell activation. The second generation includes a co-stimulating domain between TM and $\mathrm{CD} 3 \zeta$, normally derived from CD28 or 4-1BB and contains one ITAM motif. CARs of the third generation are characterized by two co-stimulating domains. CARs of the fourth generation are coupled to chemokine production. Here, an inducer for IL-12 is depicted. Fifth generation CARs contain a cytokine receptor domain leading to multipathway cascades. Cytotoxic potential, released cytokines and persistence increased from generation one to three, whereas from generation four onwards multifunctionality increased. Modified from (Tokarew et al., 2019) and (Wu et al., 2020).

\subsection{CAR T cell developments in the field of PDAC}

CAR T cells brought new hope for patients formerly believed to lack therapeutic options. This unparalleled success in the field of hematologic malignancies sparked hope to also open up novel therapeutic options in the field of pancreatic cancer.

\subsubsection{CAR T cells for PDAC: Clinical experience}

One of the most crucial factor for success of CAR T cell based therapies is the knowledge about safe and efficient targets present on the tumor cells. Suggested targets that recently have been, currently are, or in near future will be investigated in the PDAC setting, include but are not limited to mesothelin (MSLN; NCT03323944, NCT03497819, NCT03638193, NCT01897415), carcinoembryonic antigen (CEA; NCT03818165, NCT02850536, NCT02416466, NCT04037241, NCT03682744), CD133 (NCT02541370), $\quad$ CD70 
(NCT02830724), Claudin 18.2 (NCT03159819), epithelial cell adhesion molecule (EpCAM; NCT03013712), human epidermal growth factor receptor 2 (HER2; NCT02713984) mucin 1 (MUC1; NCT02587689) and prostate stem cell antigen (PSCA; NCT02744287).

CAR T cells harbor an enormous cytotoxic potential. All the CAR targets listed above are overexpressed in cancer cells but are also expressed on normal tissues, implying the risk of harming significant parts of healthy tissues. This effect is known as on-target/off-tumor toxicity. In the instance of B cell malignancy therapies targeting pan-B cell markers such as CD19 or CD20, this is acceptable, as B cell aplasia is treatable. For solid tumors, and PDAC targets in particular, less tolerable side effects have been reported. An administration of CAR T cells specific for HER2 led to fatal systemic organ failure and massive cytokine release (Morgan et al., 2010). This was attributed to HER2 expression in the lung epithelium. Maximum T cell expansion coincided with respiratory toxicities during a trial of CEA specific CAR T cells, which were also thought to be linked to CEA expression in the lung (Thistlethwaite et al., 2017). On the other hand, encouraging results could be achieved in a trial targeting CD133. Two patients experienced partial remission and only grade II toxicities, attributed to CD133 expression on hematopoietic stem cells (Wang et al., 2018). To further decrease the risk of ontarget/off-tumor toxicity, Beatty and colleagues chose a transient MSLN CAR expression method by transfecting $\mathrm{T}$ cells with CAR mRNA before infusion. In a phase I study, they observed progression free survival in two patients for up to five months and shrinkage of a liver lesion in a third patient. No adverse events were reported (Beatty et al., 2018). These examples have risen hope for PDAC patients indicating that indeed this disease can respond to CAR T cell based therapies. On the other hand it again taught the research community the importance of identifying novel target candidates including a thorough safety assessment and further optimizing CAR design in order to efficiently treat pancreatic cancer in the future. 


\subsection{Aims of the study}

PDAC is particular challenging for modern oncology. Current treatment options mainly aim at extending the remaining lifespan as a curative intervention is unlikely. Although encouraging results could be achieved in recent years, no breakthrough developments are to be expected in the near future from conventional therapy approaches. CAR T cells are a powerful new option for cancer therapy. While remarkable results have been accomplished in the setting of hematologic malignancies, several hurdles still need to be overcome for their application in the treatment of PDAC.

\subsubsection{Identification of specific and safe target candidates}

A critical stage during CAR T cell development is the choice of the right target antigen. Until today, a comprehensive assessment comparing target candidates for their specificity towards PDAC cells and studies evaluating their off-tumor expression are missing. This neglect of the safety aspects in the past already have led to fatal outcomes and severe side effects. Hence, one aim of this study was the identification of novel target candidates with high tumor specificity and low expression in healthy tissues. The specificity to tumor cells was to be investigated using an antibody based target discovery approach. This enabled an experimental assessment of tumor and healthy tissue expression and facilitated a simplified subsequent CAR design due to the direct transfer option of existing scFvs. This part of the study is investigated in "Chapter I CD318, TSPAN8 and CD66c resemble novel candidates for CAR T cell based immunotherapy of pancreatic adenocarcinoma".

\subsubsection{Generation and evaluation of target specific CAR T cells}

The optimal CAR configuration depends on multiple parameters, one important being the location of the epitope. Long spacers more efficiently target membrane proximal epitopes and vice versa. As the exact location of the target epitope is not known in most of the cases, CAR libraries have to be generated and screened. In addition, in vitro and in vivo performance of CARs may vary based on CAR design, affinity, target expression among others and have to be tested empirically. Thus, the second objective of this study was the identification of the most efficient CAR design for the newly identified target candidates. This issue is further addressed in "Chapter I - CD318, TSPAN8 and CD66c resemble novel candidates for CAR T cell based immunotherapy of pancreatic adenocarcinoma". 


\subsubsection{Development of a novel class of long spacer domains}

It is known that spacer length and structural attributes significantly influence CAR activity. However, comparative studies among spacer classes are sparse. In addition, IgG-based long spacer seem to have a risk of unspecific binding within murine models, leading to inferior functionality and contradicting results in vitro and in vivo. To overcome the inherent problems of long IgG-based spacer, the last objective of this study was the development of a novel class of CAR spacer, with similar attributes as the IgG class, but lacking their natural unspecific binding moieties. The generation and evaluation of this novel spacer class is outlined in "Chapter II - A Novel Siglec-4 Derived Spacer Improves the Functionality of CAR T Cells Against Membrane-Proximal Epitopes “. 


\section{Individual contributions to the manuscripts}

\section{Chapter I - CD318, TSPAN8 and CD66c resemble novel candidates for CAR $T$ cell based immunotherapy of pancreatic adenocarcinoma}

Figures, sub-figures, tables and experiments

All figures and sub-figures were created by me, except Figure S3 b, which was created by Dr.

Stefan Tomiuk. Dr. Stefan Tomiuk created supplementary table S1 in association with Dr. Olaf Hardt and me. All other tables were created by myself. I conducted all experiments depicted in the figures or analyzed the raw data resulting in the depicted figures.

\section{Writing}

The manuscript was written by myself with support from Dr. Olaf Hardt and input from all coauthors.

\section{Intellectual}

The study was designed and conceptualized by Dr. Olaf Hardt and Dr. Andreas Bosio in association with me. The final architecture of the manuscript was perceived by me. I designed the in vitro CAR assays and defined the parameters for read-out, in addition with the holistic scoring system of CARs and the balloon plot depiction. The in vivo studies were designed in association with Dr. Olaf Hardt and Dr. Wa'el Al Rawashdeh.

\section{Chapter II - A Novel Siglec-4 Derived Spacer Improves the Functionality of CAR T Cells Against Membrane-Proximal Epitopes}

Figures, sub-figures and experiments

All figures and sub-figures were created in collaboration with shared first author Janina Henze. I conducted the experiments depicted in the figures or analyzed the raw data resulting in the depicted figures in collaboration with shared first authors Janina Henze and Dr. Rita Pfeifer. While Janina Henze mainly conducted experimental work related to Figures 1 and 2 and Supplementary Figure 2 and 3, I conducted the experiments regarding Figure 5 and 6 . All other figures and experiments were conducted jointly.

\section{Writing}

The manuscript was written by me together with shared first authors Janina Henze and Dr. Rita Pfeifer with input from all co-authors. 


\section{Intellectual}

The manuscript was initially conceptualized by me and shared first author Janina Henze and the design of the study was further specified in association with Dr. Rita Pfeifer, Dr. Olaf Hardt, Dr. Wa'el Al Rawashdeh and Dr. Ian C. D. Johnston. Dr. Rita Pfeifer designed the novel class of Siglec spacers and shared her results using the spacers in a CD20 based model, while Janina Henze and Dr. Wa'el Al Rawashdeh shared their results from the IgG1-based spacer trials, also in a CD20 model. I recognized the pattern of activation in dependence to the target length and designed the experiments on the basis of targets with different epitope distance to the cell membrane. This enabled us to identify the Siglec-4 based CAR as highly efficient on membrane proximal epitopes with advantageous $\mathrm{T}$ cell and cytokine release phenotype. Subsequently, we drafted a patent application. 


\section{Chapter I - CD318, TSPAN8 and CD66c resemble novel candidates for CAR $T$ cell based immunotherapy of pancreatic adenocarcinoma}

Under revision at Nature Communications

\section{Title: CD318, TSPAN8 and CD66c resemble novel candidates for CAR T cell based immunotherapy of pancreatic adenocarcinoma}

Authors: Daniel Schaefer ${ }^{1,2}$, Stefan Tomiuk ${ }^{2}$, Laura N. Küster ${ }^{2}$, Wa'el Al Rawashdeh², Janina Henze $^{1,2}$, German Tischler-Höhle ${ }^{2}$, David J. Agorku², Janina Brauner ${ }^{2}$, Cathrin Linnartz ${ }^{2}$, Dominik Lock ${ }^{2}$, Andrew Kaiser ${ }^{2}$, Christoph Herbel $^{2}$, Dominik Eckardt ${ }^{2}$, Julia Schüler ${ }^{3}$, Philipp Ströbel $^{4}$, Jeannine Missbach-Güntner ${ }^{1,5}$, Frauke Alves ${ }^{1,5,6}$, Andreas Bosio ${ }^{2}$, Olaf Hardt² ${ }^{*}$.

\section{Affiliations:}

${ }^{1}$ Universitätsmedizin Göttingen, Klinik für Hämatologie und Medizinische Onkologie, Göttingen, Lower Saxony, Germany

${ }^{2}$ Miltenyi Biotec GmbH, R\&D, Bergisch Gladbach, North Rhine-Westphalia, Germany

${ }^{3}$ Charles River Discovery Research Services GmbH, Freiburg, Baden-Wuerttemberg, Germany ${ }^{4}$ Universitätsmedizin Göttingen, Institut für Pathologie, Göttingen, Lower Saxony, Germany

${ }^{5}$ Universitätsmedizin Göttingen, Institut für Diagnostische und Interventionelle Radiologie, Göttingen, Lower Saxony, Germany

${ }^{6}$ Max-Planck-Institut für Experimentelle Medizin, Translational Molecular Imaging, Göttingen, Lower Saxony, Germany

\section{One Sentence Summary}

We identified target candidates for CAR T cell based treatment of pancreatic cancer using novel screening techniques followed by in vitro and in vivo functionality testing. 


\begin{abstract}
A major roadblock prohibiting effective cellular immunotherapy of pancreatic ductal adenocarcinoma (PDAC) is the lack of suitable tumor-specific antigens. To address this challenge, we combined flow cytometry screenings, bioinformatic expression analyses and a newly developed cyclic immunofluorescence platform. We identified CLA, CD66c, CD318 and TSPAN8 as novel target candidates among 371 antigens and generated 32 CARs specific for these molecules. CAR T cell activity was evaluated in vitro based on target cell lysis, $\mathrm{T}$ cell activation and cytokine release. Promising constructs were evaluated in vivo. CAR T cells specific for CD66c, CD318 and TSPAN8 demonstrated efficacies ranging from stabilized disease to complete tumor eradication with $\mathrm{CD} 318$ being the most promising candidate for a clinical translation based on functionality and its predicted safety profile. This study reveals novel target candidates for CAR $\mathrm{T}$ cell based immunotherapy of PDAC together with a functional set of CAR constructs specific for these molecules.
\end{abstract}

\title{
Introduction
}

Pancreatic cancer is a devastating disease. The 5-year overall survival rates have merely changed for the past decades and it is currently the fourth leading cause of cancer related deaths in Western countries $(1,2)$. Surgery still is the only potentially curative treatment, but only around $20 \%$ of patients show a resectable disease stage at diagnosis (3). Median overall survival with state-of-the-art treatment ranges from 26 month for patients with resectable disease to less than six months when already metastatic (4). Thus, there is an unmet need for new therapeutic options. A new and promising therapeutic approach are chimeric antigen receptor (CAR) $T$ cells. CAR T cells showed unprecedented efficacies in the treatment of B cell malignancies $(5,6)$. They typically rely on Pan-B cell antigens such as CD19 or CD20 and do not discriminate between healthy and tumor cells. As a consequence, all B cells are depleted, which is regarded as an acceptable side effect since it is otherwise well tolerated (7). This is not the case for almost all target antigens in solid tumors until today and remains one of the central problems of solid tumor CAR T cell therapies. Prominent candidates among the targets which are currently under investigation in clinical trials for pancreatic cancer are carcinoembryonic antigen (CEA), human epidermal growth factor receptor 2 (HER2), mucin 1 (MUC1), prostate stem cell antigen (PSCA), prominin 1 (PROM1), epidermal growth factor receptor (EGFR) and mesothelin (MSN) (8). These target candidates all have in common their shared expression on malignant and healthy tissues and toxicities in humans were already reported for HER2 and CEA. While administration of HER2 specific CAR T cells ended fatal for the patient (9) the use of CAR T 
cells against CEA caused only mild toxicities but also very limited efficacy $(10,11)$. These examples underline how important the aspect of safety is, which in case of CAR T cells comes with tumor specificity and off-tumor expression in dispensable cell types only.

Until today, a broad and systematic target antigen screen for CAR T cell therapy of pancreatic cancer that compares the specificity of a multitude of target candidates and their off-tumor expression has not been reported. Likewise, empirical studies needed to determine an optimal CAR design on suitable targets for this disease are also scarce (12).

Hence, we aimed to close this gap and present here a systematic approach for CAR target screening that first narrowed down the field of target candidates from 371 to 50 by flow cytometric analysis of 17 pancreatic cancer patient derived xenograft (PDX) models. We investigated further the RNA and protein expression profiles of these target candidates which are available in public online data banks. We ranked the candidates in dependence of their expression in different healthy tissues and cell types. In addition, we examined the expression of a multitude of these target candidates within primary pancreatic cancer tissues from patients using a newly developed cyclic immunofluorescence imaging platform. This new and powerful technique enabled us to survey expression profiles of several dozens of antigens on the very same tissue section. We finally verified these results using flow cytometry on seven additional primary PDACs. Based on these results, we designed 32 CARs specific for the four most promising target candidates, CLA, CD66c, TSPAN8, and CD318 with varying spacer lengths and $\mathrm{scFv}$ orientations. We empirically evaluated the CAR constructs in terms of cytotoxicity, cytokine release and cell phenotype profile. CAR constructs that performed best in vitro were then examined in two independent preclinical mouse xenograft models and evaluated for their expression on healthy tissues by cyclic immune fluorescence and flow cytometry resulting in promising candidates for future clinical trials.

\section{Results}

Identification of novel PDAC cell surface target candidates for CAR T cell based immunotherapy

As pancreatic ductal adenocarcinoma (PDAC) attributes to around $85 \%$ of all pancreatic cancer cases, we decided to use PDX models of PDAC for initial candidate identification due to their good availability and proven predictivity for the disease (13). A scheme of the workflow for identification of novel target candidates applied in this study is depicted in Fig. S1. Overall, we analyzed 17 independent PDX models representing 15 different mutational backgrounds. 
Initially, we screened two PDX models representative of PDAC concerning histology, mutational profile and characteristic response to standard-of-care drugs (Charles River personal communication) using a commercially available antibody array containing antibodies specific for 371 surface antigens, including antigens already under clinical investigation for CAR T cell based treatment of PDAC, such as HER2, MUC1, PROM1 and CEA. We found 105 antigens to be expressed on more than $10 \%$ of the PDX cells on at least one of the PDX. We then used antibodies specific for these 105 antigens and measured their expression on two additional representative PDX models followed by a manual exclusion of non-suitable target candidates, such as HLA molecules which were present in the pre-set screening plates. We measured the remaining 50 surface antigens, which were expressed in at least $20 \%$ of all tumor cells of at least three out of four PDX models, on 13 more xenografts (Fig. 1a, Fig. S2). Remarkably, MUC1 and HER2 did not match these criteria. A family of proteins that was expressed on many tumor cells of the PDX models was the one of the tetraspanins. Prominently expressed members were CD9, CD63, CD82 and CD151. Another family that showed expression on many tumor cells throughout the different PDX models was the CEA family. Its members CEACAM1, CEACAM3, CEACAM5 and CEACAM6 could all be recognized by the pan-reactive CD66acde antibody. However, the expression pattern of CD66acde was paralleled by the expression of CD66c alone and based on the higher specificity of the single molecule binder we chose this one for further evaluation. Unexpectedly, we found the cutaneous lymphocyte antigen (CLA) expressed on all but one xenograft. CLA is described as a binding epitope of the antibody clone HECA 452 and includes a sialyl-Lewis x glycan structure $(14,15)$. So far, CLA was only observed to be expressed on subsets of leukocytes (14,16-19). This is in two ways an interesting finding: it was never described before to be expressed on pancreatic cancer cells and was so far only reported to be expressed on sub sets of certain cell types, which could mean no essential tissues may be harmed when targeted by CAR T cells.

CD66c, CD318, TSPAN8 and CLA exhibited restricted off-tumor expression in human tissues

Next, we prioritized the 50 surface structures from our antibody screen with respect to their offtumor expression. We assigned the corresponding genes to their respective antigens. For some instances multiple genes had to be assigned to a single antibody. For example CD66acde represents CEACAM1, CEACAM3, CEACAM5 and CEACAM6. In cases like this, each gene was investigated independently. In case an antibody was specific for a glycostructure we assigned the respective backbone protein to it (for example CLA can be a glycostructure on 
SELPLG (14)), if possible. Subsequently, we extracted RNA and protein expression data of the assigned genes from the following data sources: Human Protein Atlas (20), ProteomicsDB (21), Human Proteome Map (22) and GTEx. Next, we defined rankings independently for each dataset. Detailed information about the ranking procedure can be found in the Materials and Methods part. In brief, genes of each data set were ranked by the total number of tissues/cell types it was expressed in as well as their overall expression levels. Subsequently, we calculated a rank sum that mirrors the overall expression throughout all data sources (Tab. S1, Fig. S3). The final rank was then calculated based on the quotient of the rank sum of the target candidate divided by the number of data sources it was found in. As a consequence, target genes expressed in a low number of tissues were prioritized as they are expected to cause lower potential offtumor effects.

All four members of the CEA family that are bound by the CD66acde antibody exhibited only restricted expression over several tissues (Tab. S1, Fig. S3). Other target candidates that appeared top ranked were the Rh blood group polypeptides RHD and RHCE (CD240DCE), the C-C chemokine receptor type 5 (CCR5, CD195), CXC chemokine receptor 3 (CXCR3, CD183) or CUB domain-containing protein 1 (CDCP1, CD318). Remarkably, from the five tetraspanins found prominently expressed on the xenografts only TSPAN8 remained in the 20 top ranked candidates, showing that TSPAN8 has the most restricted expression profile of them. Furthermore, we found SELPLG as the protein backbone of CLA also belonging to the 20 best ranked candidates.

In addition, we included MSN and EGFR into our bioinformatic analysis as they were not present in the antibody library, whereas other candidates currently evaluated in clinical trials, such as CEA, HER2, CD133 and MUC1 were included in our screen, showing rankings similar to our subsequent target candidates (Tab. S1, Fig. S3). As an independent validation of our bioinformatics based strategy, we applied an algorithm according to the one developed by Perna et al. (23) resulting in a comparable prioritization (Tab. S1).

\section{Validation of CD66c, CD318, TSPAN8 and CLA expression and specificity}

An important information, that is lost during the processing of samples for RNA or protein expression analysis is the spatial distribution of target candidate expression within the tissue. Strong mRNA expression values derived from small populations of cells that may be acceptable with respect to toxicities are intermingled with the rest and turn suitable targets to false negatives. To overcome these problems and to gain a better understanding of the target candidate expression in primary PDACs in situ, we used a newly developed cyclic 
immunofluorescence imaging platform. This system operates by iterative fluorescence staining, imaging and signal erasure, enabling the operator to identify the expression of dozens of targets on the very same tissue section. We performed two runs of cyclic immunofluorescence imaging on two different PDAC tumor tissues with 107 and 98 markers, respectively (Tab. S2). These markers were a selection of the ones used on the PDX models plus markers to dissect known cell lineages. Our observations were centered on identifying candidates showing colocalization with tumor cells and no or low expression on non-tumor cells. Target candidates that were ranked promising through bioinformatics and showed specific expression on tumor cells from patients were CLA, TSPAN8, CD318 and CD66c (Fig. 1b, Tab. S1, Fig. S3). Other candidates such as CD240DCE, CD195 and CD183, which were ranked well before exhibited strong expression on non-tumor cells. Some candidates appeared to be specific to the tumor cells, although they did not belong to the group of high ranked targets, such as CD49c, CD73, CD104 and CD142 suggesting a generic epithelial reactivity (Fig. 1b). CD51, CD59 and CD107a had low bioinformatic ranks and presented massive expression offside of tumor cells, nicely confirming the value of this approach.

These findings led to the decision to further verify the specificity of CD49c, CD66c, CD73, CD104, CD142, CD318, CLA and TSPAN8 on primary pancreatic tumor cells using flow cytometry. We dissociated seven human PDAC specimens and gated either on tumor cells $\left(\mathrm{EpCAM}^{+}\right)$, leukocytes $\left(\mathrm{CD} 45^{+}\right)$or other cells (double negative). It was shown that these eight candidates indeed showed a significant enrichment of expression on tumor cells (Fig. 1c, Fig. S4). We decided to continue with the generation of CARs specific for CLA, CD66c, CD318 and TSPAN8, since the bioinformatical rankings indicated a higher health threat for CD49c, CD73, CD104 and CD142 (Tab. S1, Fig. S3). 


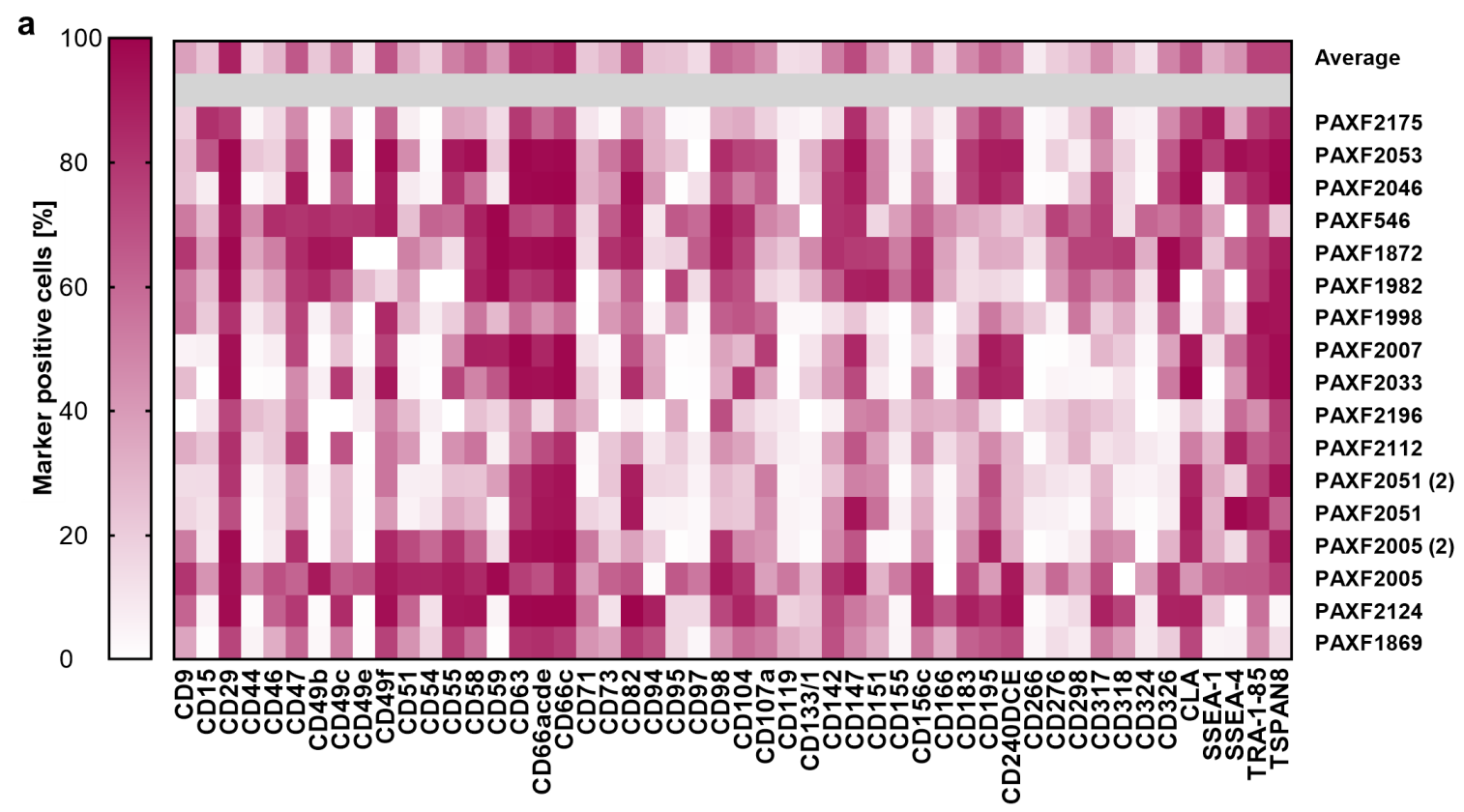

b

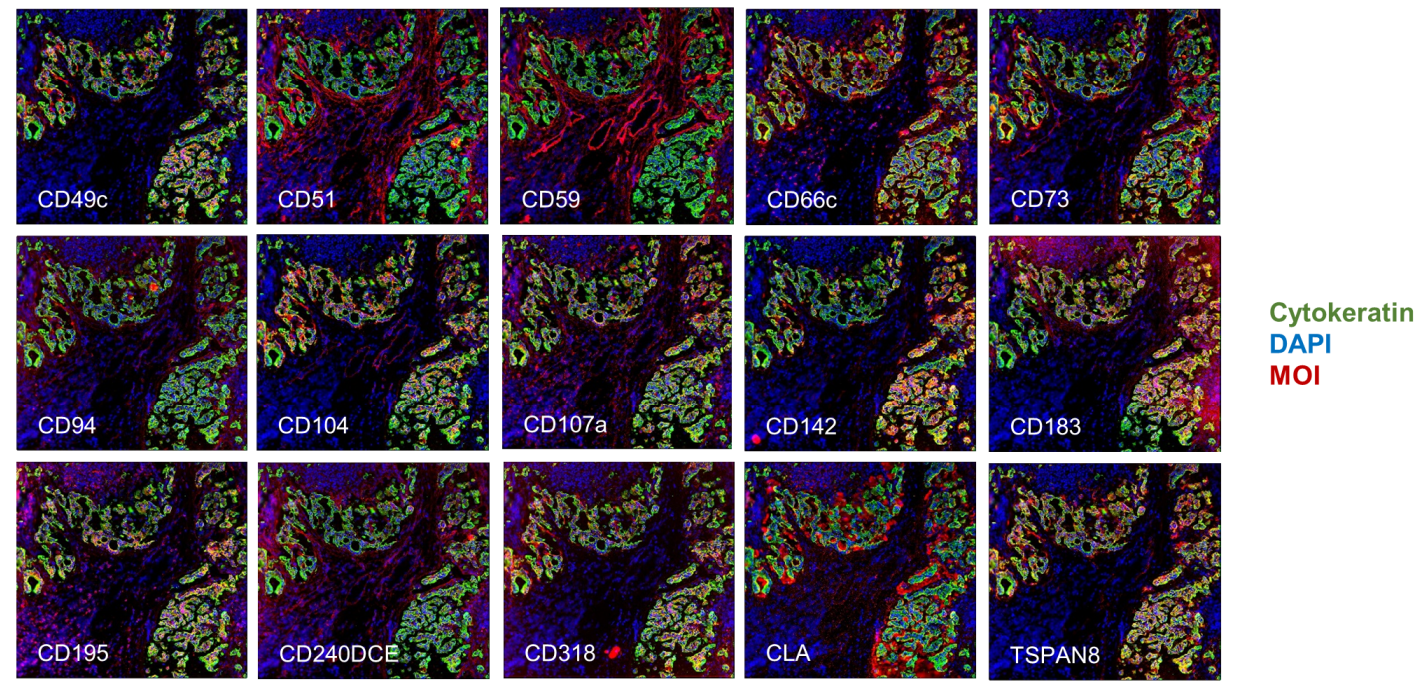

c

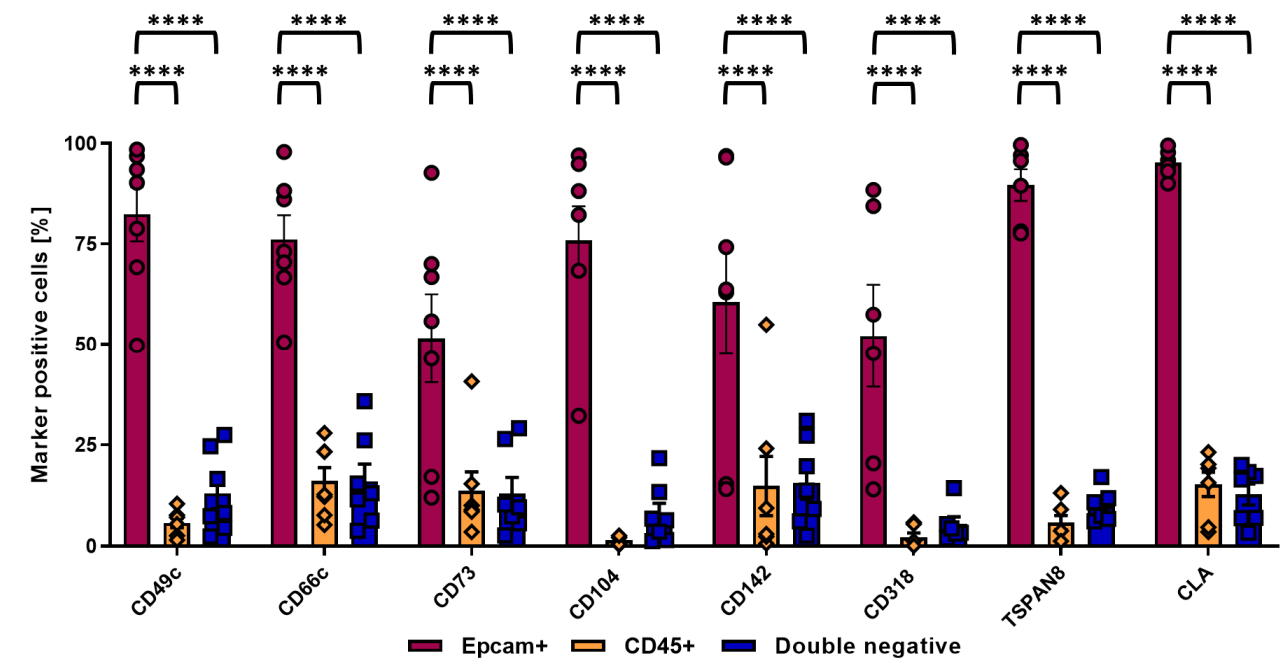


Fig. 1: Identification of novel PDAC CAR target candidates. (a) Top 50 antigens expressed on the cell surface of PDAC PDX models as determined by flow cytometry based screening of a 371 monoclonal antibody panel. (b) Selection of 15 cyclic immunofluorescence images of a representative human PDAC tissue to evaluate coexpression of the marker of interest (MOI) with cytokeratin-positive tumor cells. (c) Flow cytometric analysis of expression for eight target candidates on primary human PDAC samples. Data represent mean \pm s.e.m. $(\mathrm{n}=7$, ****P $<0.0001$ determined through two-way ANOVA with Tukey's test).

CLA specific CARs are dysfunctional, while CARs specific for CD66c, CD318 and TSPAN8 induce potent activation, cytokine release and antitumor efficacy in vitro

After identification of the four target candidates CLA, CD66c, CD318 and TSPAN8, we designed a CAR library with varying spacer lengths (XS, S, M and L) and scFv orientations ( $\mathrm{V}_{h}-\mathrm{V}_{1}$ and $\mathrm{V}_{1}-\mathrm{V}_{\mathrm{h}}$ ) (Fig. 2a). It has been shown that these elements are critical for CAR function $(12,24)$. All CARs shared a second generation design with CD3 $\zeta$ and 4-1BB as stimulatory domains and a CD8 $\alpha$ transmembrane domain. The CAR sequence was connected to the sequence of the extracellular domain of the Low-affinity nerve growth factor receptor (LNGFR) via a P2A site, to be used as a reporter protein. In total, eight CAR constructs with differing spacer lengths and varying $\mathrm{scFv}$ orientations were generated for each candidate.

Following the design and cloning of the CAR containing plasmids, the general ability of the CAR constructs to be expressed and localized to the cell surface was assessed in transfected HEK293T cells (Fig. S5). The majority of constructs displayed LNGFR expression, indicating a functional CAR cassette. In contrast, the anti-Fab staining differed greatly among spacer lengths and scFv specificities (Fig. S5). Possible explanations could be a decreased stability as compared to LNGFR, suboptimal folding or cell surface localization characteristics of the artificial sequences that lead to an early intracellular degradation or sub-optimal binding of the polyclonal anti-mouse Fab. Most strikingly, anti-Fab staining of most of the M sized CARs showed substantial mal-expression. These findings reasoned us to exclude M sized CARs from further assessments.

Following this, we set up an in vitro co-culture system to validate functionality of the remaining CARs. We generated GFP-Luciferin positive PDAC cell lines from established AsPC1 and BxPC3 PDAC lines as well as one cell line we derived directly from one of the primary tissues (PanCa0201). We co-cultured the target cell lines with CAR T cells in effector-to-target (E:T) ratios of 5:1,2:1 and 1:1 over 6 days. We measured cytotoxicity as a function of decrease in viable target cell confluence. Samples of the supernatant were taken after 2 days of co-culture for cytokine release measurements and $\mathrm{T}$ cell activation/exhaustion marker expression was measured after 2 or 6 days. We considered GM-CSF, IL-2, TNF- $\alpha$ and INF- $\gamma$ as the cytokines which have the highest value in terms of CAR $\mathrm{T}$ cell activity. The examined $\mathrm{T}$ cell markers 
were PD-1, LAG-3, 4-1BB, TIM-3 and LAG-3/4-1BB co-expression. To ensure interexperiment comparability, we normalized the values to the highest of each assay. All CAR T cells were evaluated for unspecific activation and induction of tonic signaling in a co-culture with HEK293T cells, not expressing any of the target antigens (Fig. S6). Furthermore, general activation ability was tested stimulation T cells with PMA/Ionomycin (Fig. S6b, Tab. S3).

All anti-CLA CARs performed much worse as compared to their counterparts for the other antigens (Tab S3, Fig. S7a). They displayed only weak killing and almost no cytokine release or marker upregulation. We think the cause of this was CLA to be a $\mathrm{T}$ cell self-antigen upregulated upon activation (Fig. S8). As shown above, the expression levels of CLA specific CARs in HEK293T cells were very low. This in combination with self-antigenicity may lead to binding of CAR and target already intracellular and in consequence to degradation of this complex. For CD66c CARs the S and XS spacer performed better in both scFv orientations as compared to the $\mathrm{L}$ spacer CARs. The $\mathrm{V}_{\mathrm{l}}-\mathrm{V}_{\mathrm{h}} \mathrm{scFV}$ orientation performed superior to the $\mathrm{V}_{\mathrm{h}}-\mathrm{V}_{1}$ orientation. The CD318 CARs featured similar behavior, with the XS and S spacers being superior to the longer $\mathrm{L}$ spacer. For $\mathrm{CD} 318$ the $\mathrm{V}_{\mathrm{h}}-\mathrm{V}_{1}$ conformation appeared to be more functional. These findings were contrasted by the CARs specific for TSPAN8, where the long spacer exhibited better cytotoxicity. The results of all in vitro functionality assays can be found in Tab. S3.

Subsequently, we directly compared the best performing CARs based on the highest values of cytotoxicity, cytokine release and marker upregulation (Fig. 2b, Tab. S4). We chose the XS and S CARs in the $\mathrm{V}_{1}-\mathrm{V}_{\mathrm{h}}$ orientation for CD66c, the XS and S CARs in the $\mathrm{V}_{\mathrm{h}}-\mathrm{V}_{1}$ orientation for $\mathrm{CD} 318$, and the $\mathrm{S}$ and $\mathrm{L}$ spacer CARs in the $\mathrm{V}_{\mathrm{h}}-\mathrm{V}_{1}$ conformation as well as the $\mathrm{V}_{1}-\mathrm{V}_{\mathrm{h}} \mathrm{L}$ spacer CAR for TSPAN8. In this direct comparison, the CD66c S spacer CAR outperformed the XS spacer CAR. Both CD318 CARs showed fast and efficient target cell killing with almost complete lysis using BxPC3 and the fastest kinetic using AsPC1 cells (Fig. 2c,d, Fig. S7b,c). A slight difference in performance only appeared based on cytokine release and activation marker upregulation. The XS spacer released less cytokines but exhibited improved activation marker upregulation. All other CARs showed cell lysis between $40 \%$ to $60 \%$ within the first $48 \mathrm{~h}$ following an outgrowth control until $160 \mathrm{~h}$ (Fig. 2c, Fig. S7b). The highest activation of TSPAN8 specific CARs showed the $\mathrm{S}$ spacer in $\mathrm{V}_{\mathrm{h}}-\mathrm{V}_{1}$ orientation (Fig 2b). As a consequence, we chose to evaluate the CAR constructs CD66c S Vl-Vh, CD318 XS Vh-V1, TSPAN8 S and L $\mathrm{V}_{\mathrm{h}}-\mathrm{V}_{1}$ for in vivo functionality in two pre-clinical models. 
a

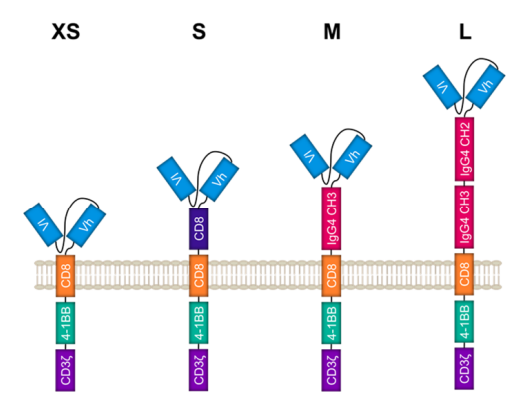

c

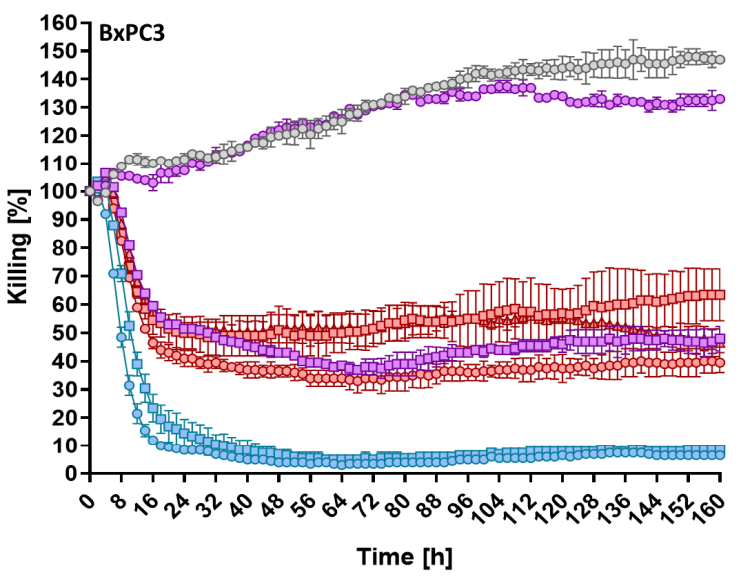

d
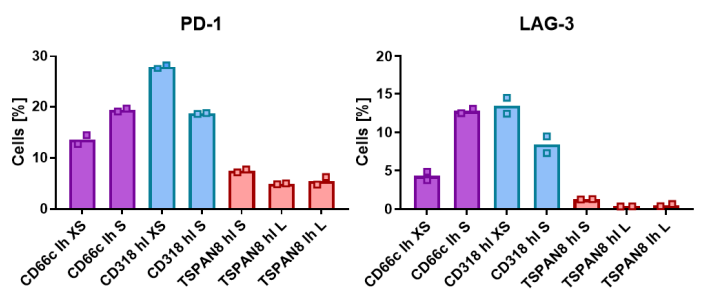

4-1BB

LAG-3/4-1BB
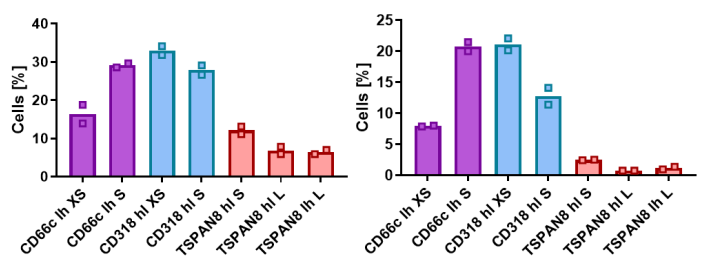

TSPAN8 $\mathbf{V}_{\mathbf{l}}-\mathbf{V}_{\mathrm{h}} \mathbf{L}$

TSPAN8 $\mathrm{V}_{\mathrm{h}}-\mathrm{V}_{\mathrm{I}} \mathrm{S}$

TSPAN8 $\mathrm{V}_{\mathrm{h}}-\mathrm{V}_{\mathrm{I}} \mathrm{L}$

CD66c $V_{\mathrm{l}}-\mathrm{V}_{\mathrm{h}} \mathrm{XS}$

CD66c V $-V_{h} S$

CD318 V $\mathrm{h}_{\mathrm{h}}-\mathrm{V}_{1}$ XS

CD318 V $\mathrm{h}_{\mathrm{h}} \mathrm{V}$

$(4,8,8)$

$(4,8,8)$

$(4,8,8)$

$(4,8,8)$

$(4,8,8)$

$(4,8,8)$

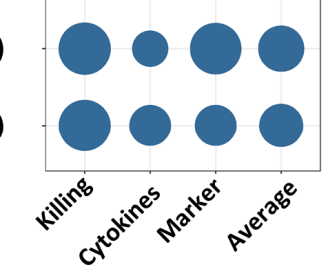

-O- Mock

$-0-\quad \operatorname{cD} 66 c V_{1}-V_{h} X S$

$\rightarrow \quad$ CD66c V V $-V_{h} S$

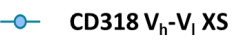

$\rightarrow-\quad C_{18} 18 V_{h}-V_{l} S$

-O- TSPAN8 $\mathrm{V}_{\mathrm{h}}-\mathrm{V}_{\mathrm{l}} \mathrm{S}$

$\neg-$ TSPAN8 $\mathrm{V}_{\mathrm{h}}-\mathrm{V}_{\mathrm{l}} \mathrm{L}$

$\triangle \quad$ TSPAN8 $\mathrm{V}_{\mathrm{l}}-\mathrm{V}_{\mathrm{h}} \mathrm{L}$
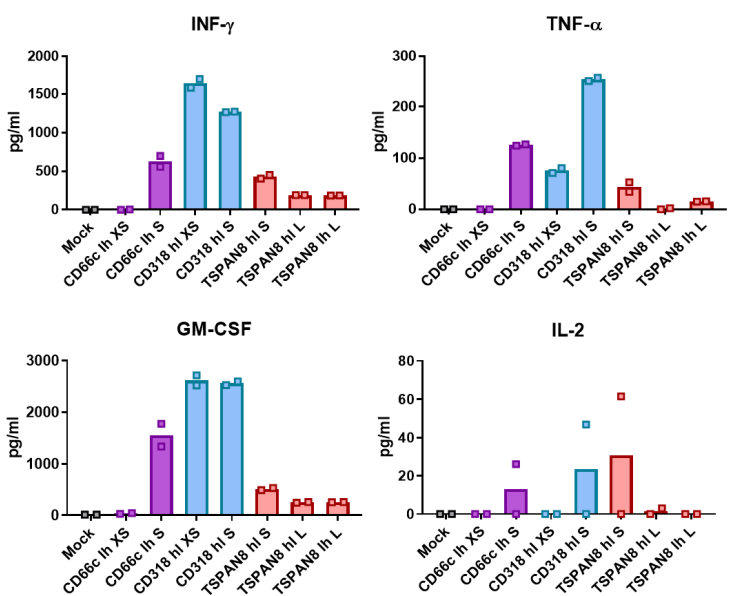

IL-2

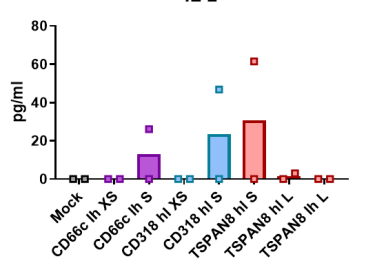

Fig. 2: Generation of target candidate specific CAR constructs and evaluation of in vitro functionality. (a) Scheme of the generation of our CAR library. The combination of four backbones differing in spacer length with two scFv orientations and four target candidate specificities resulted in 32 constructs. (b) Average relative target cell killing, cytokine release, marker upregulation and overall performance (displayed as circle size) of the selected best performing CARs from the initial screening assays (y axis). The number of replicates is indicated in parentheses behind the construct name. (First value $=n$ of killing assays, second value $=n$ of cytokine release assays, third value $=\mathrm{n}$ of marker upregulation measurements). (c) Representative result for the kinetics of BxPC3 target cell killing by the selected CAR constructs. (d) Representative results showing cytokine release and activation marker expression patterns upon co-culture with BxPC3 target cells. Shown are the means \pm s.e.m. $(n=2)$. Activation marker expression was measured at end point of cytotoxicity assay, cytokine release patterns were measured after $48 \mathrm{~h}$. 


\section{CAR T cells specific for the novel target candidates exhibit potent anti-tumor responses in}

vivo

To evaluate the most promising CAR candidates in a preclinical setting, we engrafted $\mathrm{Luc}^{+}$ AsPC1 cells subcutaneously in NSG mice. Tumor growth kinetics were evaluated by caliper and bioluminescence (BLI) measurements (Fig. 3a). After the first tumors reached a size of $25 \mathrm{~mm}^{2}$, we injected $5 \times 10^{6} \mathrm{CAR} \mathrm{T}$ cells or untransduced $\mathrm{T}$ cells intravenously. Mock T cells exhibited no therapeutic benefit over the untreated group (Fig. S9a). All mice treated with Mock $\mathrm{T}$ cells were sacrificed latest 12 days post $\mathrm{T}$ cell injection due to appearance of ulceration in tumors (Fig. S9b). Remarkably, all tumors irrespective of the treatment grew in terms of size until 9 days post $\mathrm{T}$ cell injection, although for some animals the BLI signal decreased already after six days (Fig. 3b,c-d). We could verify that this drop in BLI signal and increase in size was caused by tumor cell lysis initiated trough massive CAR T cell and macrophage infiltration (Fig. 4c). Complete and fast tumor eradication was observed in the CD318 XS CAR treated group. The TSPAN8 S CAR showed a slower therapeutic kinetic but achieved tumor clearance in three mice and almost complete clearance in one mouse. The TSPAN8 L CAR exhibited heterogeneous response with three complete responders and two mice showing stable tumor burden. The CD66c S spacer performed poorly in comparison but still stabilized the tumor burden in all animals (Fig. 3b,c). Flow cytometric analysis of spleen CAR T cells revealed that the groups CD318 XS and TSPAN8 S had the highest CAR T cell counts at the end of the experiment correlating with the highest anti-tumor activity (Fig. 4a). In addition, the two lowresponder mice showed the lowest CAR T cell count inside the TSPAN8 L group. However, the comparable cell counts of the CD66c group showed that the CAR T cell number alone is not sufficient to explain the efficacy. Flow cytometric and microscopy analyses of the tumors ex vivo showed that unresponsiveness was not linked to target downregulation (Fig.4c,d, Fig. S10). Mice treated with CD318 CARs showed increased infiltration of T cells, CAR T cells and macrophages as compared to those treated with CD66c CAR T cells, whereas the composition of central memory (ТСм) and effector memory (ТЕм) phenotypes did not differ significantly among the groups (Fig. 4b). 


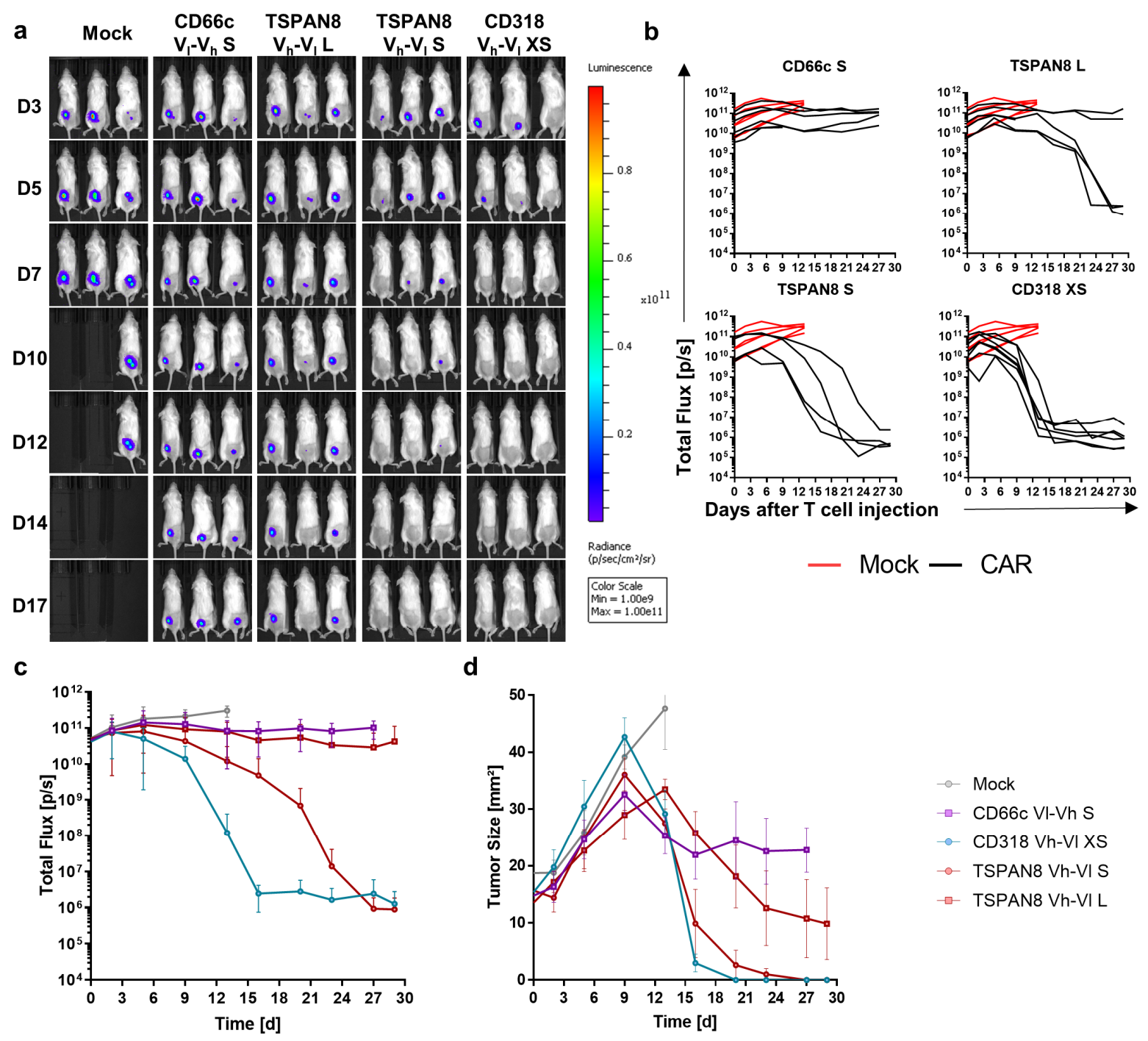

Fig. 3: Evaluation of CAR $\mathrm{T}$ cell in vivo functionality in an AsPC1 xenograft model. (a) Representative bioluminescence images of tumor bearing NSG mice. Tumors were induced by subcutaneously transplanting Luciferase expressing AsPC1 cells (Color scale for all images, $\min =1 \times 10^{9}, \max =1 \times 10^{11}$ ). Mice were randomized

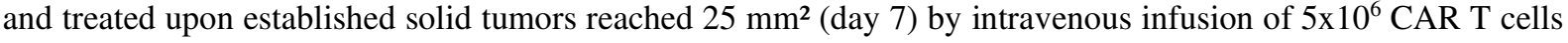
or Mock T cells. (b) Development of tumor burden for individual mice treated either with Mock T cells or with the respective CAR T cells (Mock: $n=6$, CD66c S: $n=5$, CD318 XS: $n=7$, TSPAN8 S: $n=4$, TSPAN8 L: $n=$ 6). (c) Average bioluminescence signal \pm s.e.m. of the respective treatment groups (n equal to b). (d) Average tumor size \pm s.e.m. of the respective treatment groups (n equal to b). 

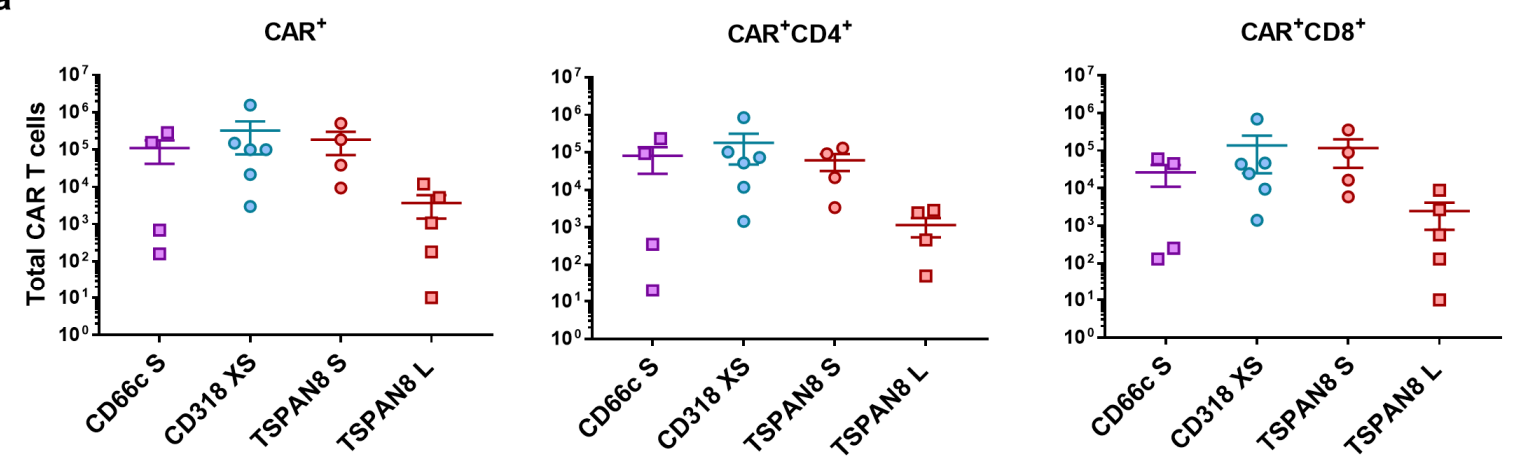

b

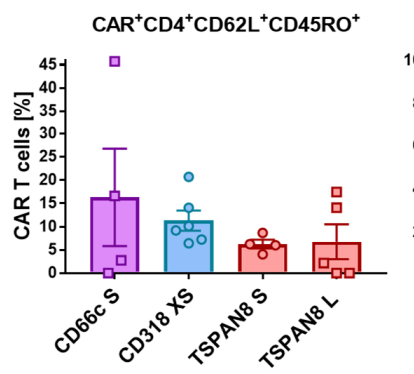

C
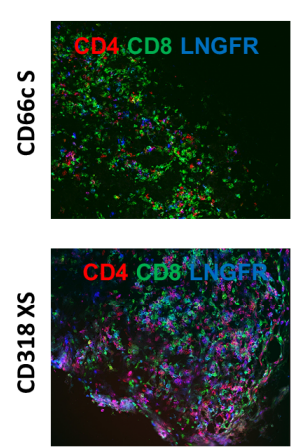
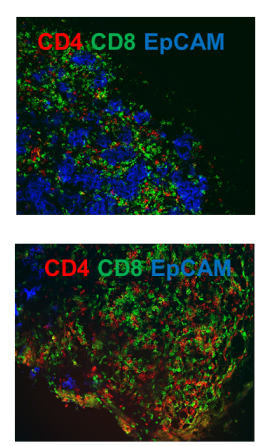
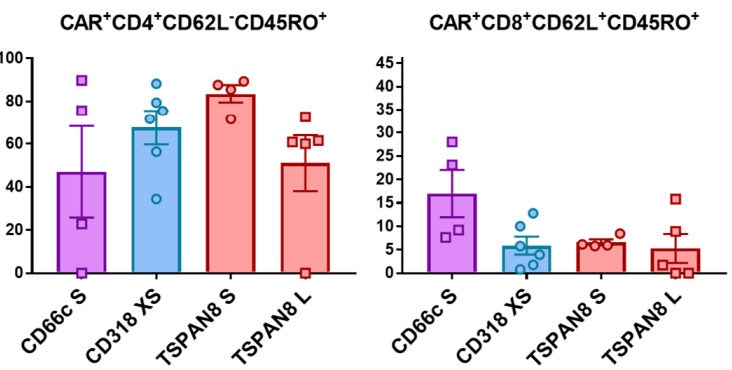

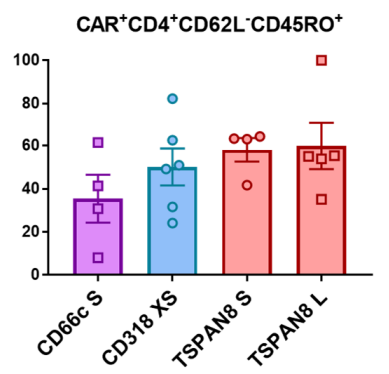

d
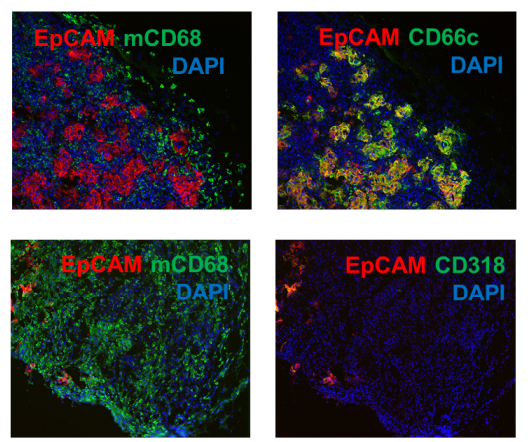

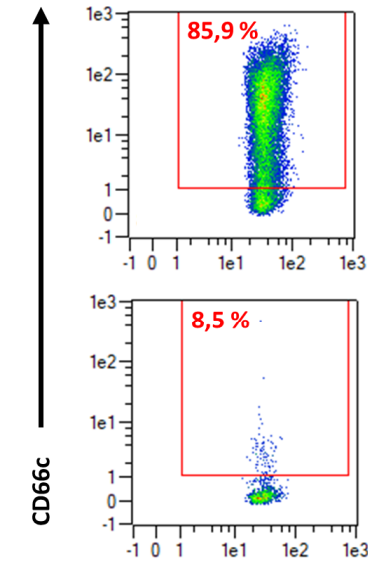

FSC

Fig. 4: $E x$ vivo analysis of the T cell phenotype and AsPC1 derived tumor tissues upon treatment with CAR T cells. (a) Number of CAR $T$ cells in the spleen at the end of the experiment $27 \mathrm{~d}$ post CAR $\mathrm{T}$ cell injection (CD66c S: $\mathrm{n}=4$, CD318 XS: $\mathrm{n}=6$, TSPAN8 S: $\mathrm{n}=4$, TSPAN8 L: $\mathrm{n}=5$ ). (b) Phenotype of CAR T cells in the spleen at the end of the experiment $27 \mathrm{~d}$ post CAR $\mathrm{T}$ cell injection, as demonstrated by the percentage of $\mathrm{T}_{\mathrm{CM}}$ and $\mathrm{T}_{\mathrm{EM}}$ (n equal to a). All data are shown as mean \pm s.e.m. (c) Representative immunofluorescence images of (CAR) T cell tumor infiltration, macrophage tumor infiltration and target expression (CD318 XS tumor 9 days post CAR $\mathrm{T}$ cell injection, CD66c S tumor 27 days post CAR T cell injection). (d) Density plots of a dissociated AsPC1 xenograft showing CD66c expression 35 days post injection of $5 \mathrm{e}^{6} \mathrm{CD} 66 \mathrm{c} \mathrm{S} \mathrm{V}-\mathrm{V}_{\mathrm{h}}$ CAR $\mathrm{T}$ cells (top) and the unstained control (bottom). 
To confirm the robustness of our results, we challenged these CAR constructs in a second preclinical setting using BxPC3 and an increased dose 1x107 CAR T cells (Fig. 5a-c). As seen previously, Mock T cells lacked therapeutic effect (Fig. S9). The CD318 XS CAR again achieved a complete tumor eradication showing a fast kinetic. TSPAN8 S CARs showed a higher therapeutic functionality as the $\mathrm{L}$ spacer but both only induced stable tumor burden. In this model, no therapeutic effect was observed for the CD66c S CARs, correlating with the poor outcome in the first study. Reduced efficacy of the TSPAN8 CARs could be caused by reduced target expression of this tumor model as compared to the AsPC1 xenograft (Fig. S10). As observed for AsPC1, reduction of the tumor size was delayed as a result of $\mathrm{T}$ cell and macrophage infiltration (Fig. 6c). Analysis of the total T cell counts in the spleen at the end of the study confirmed that higher CAR T cell counts correlated with higher efficacy, again CD66c CAR T cells being the exception (Fig. 6a). T cell phenotyping revealed a decrease in the TCM compartment (Fig. 6b) within the CD66c S CAR T cells as compared to all other CAR treated groups.

In conclusion, we could verify therapeutic efficacy of our CAR constructs at different therapeutic magnitudes ranging from tumor control to total tumor eradication and revealed an overall high correlation among both in vivo studies. In addition, low-responsiveness - as seen for the CD66c construct - was not linked to target downregulation but rather to decreased CAR $\mathrm{T}$ cell infiltration or residency at the tumor. 

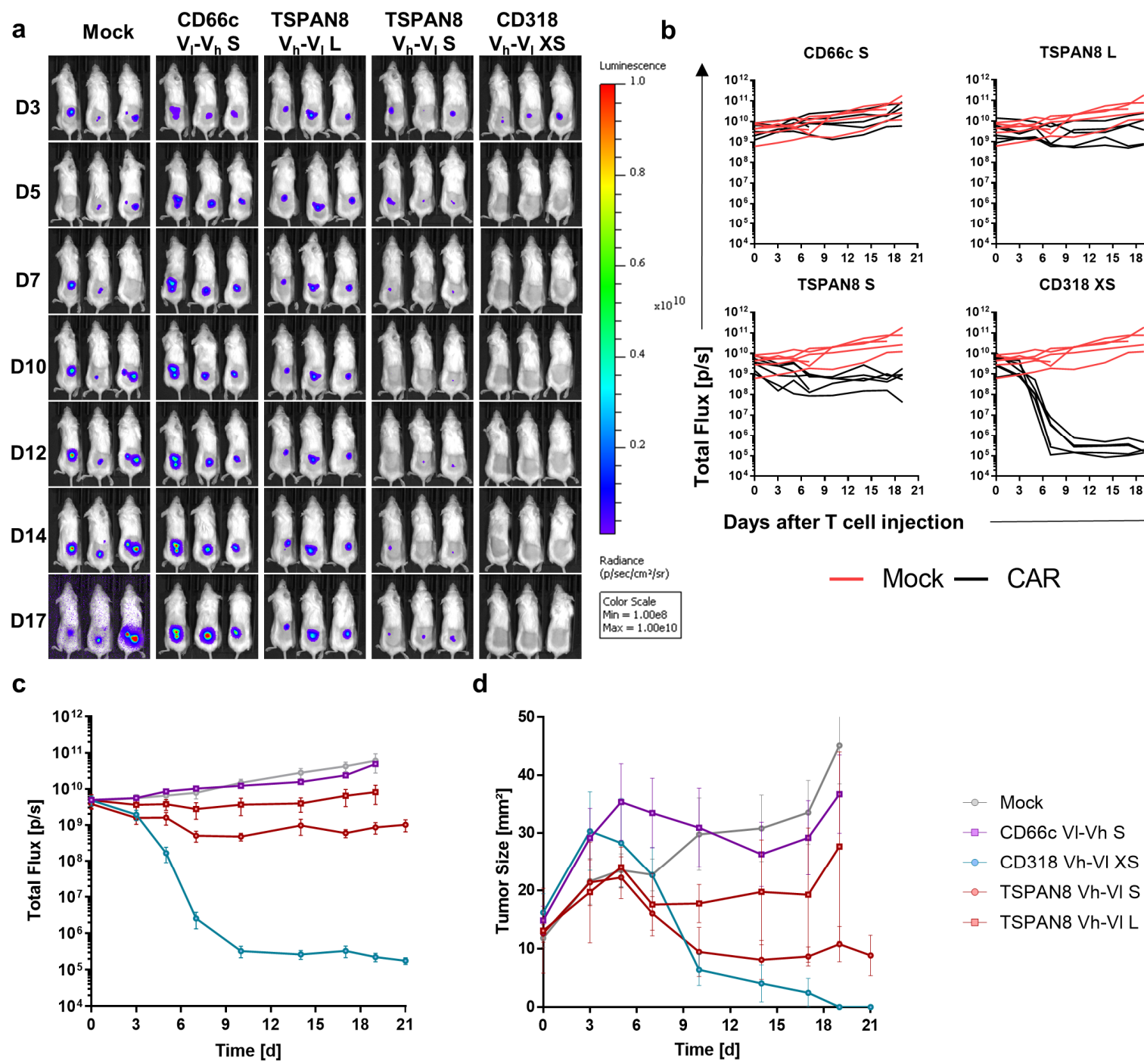

Fig. 5: Evaluation of CAR $T$ cell in vivo functionality in a BxPC3 xenograft model. (a) Representative bioluminescence images of tumor bearing NSG mice. Tumors were induced by subcutaneously transplanting Luciferase expressing BxPC3 (Color scale for all images, $\min =1 \times 10^{8}$, $\max =1 \times 10^{10}$ ). Mice were randomized and treated upon established solid tumors reached $25 \mathrm{~mm}^{2}$ (day 15) by intravenous infusion of $1 \times 10^{7}$ CAR T cells or Mock T cells. (b) Development of tumor burden for individual mice treated either with Mock T cells or with the respective CAR T cells (Mock: $n=6$, CD66c S: $n=6$, CD318 XS: $n=6$, TSPAN8 S: $n=6$, TSPAN8 L: $n=6$ ). c, Average bioluminescence \pm s.e.m. of the respective treatment groups (n equal to b). (d), Average tumor size \pm s.e.m. of the respective treatment groups (n equal to b). 

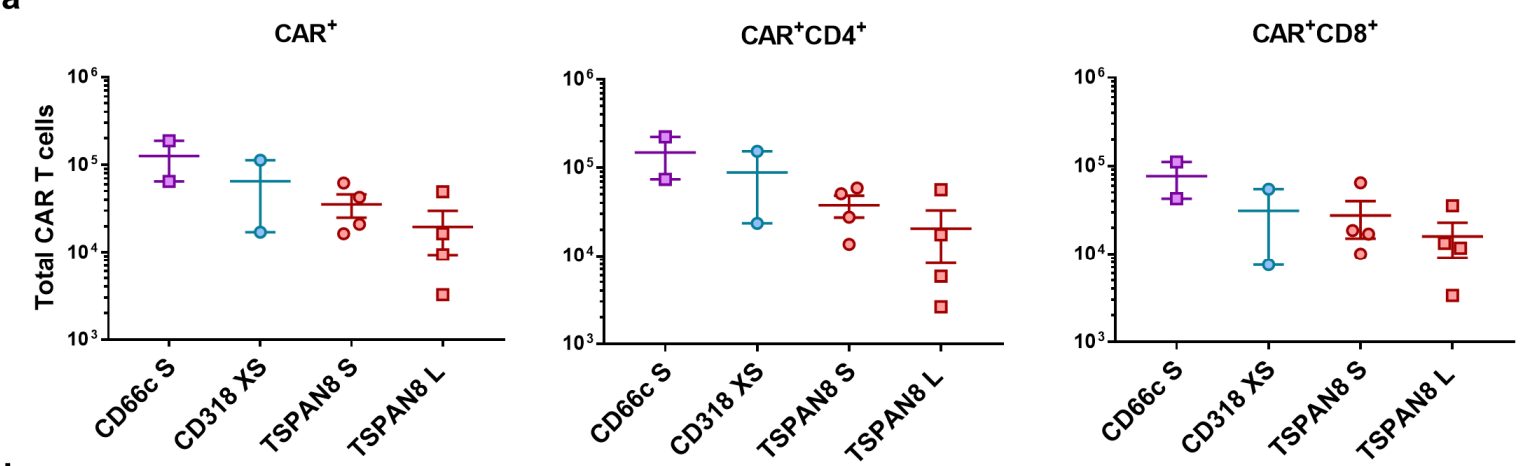

b

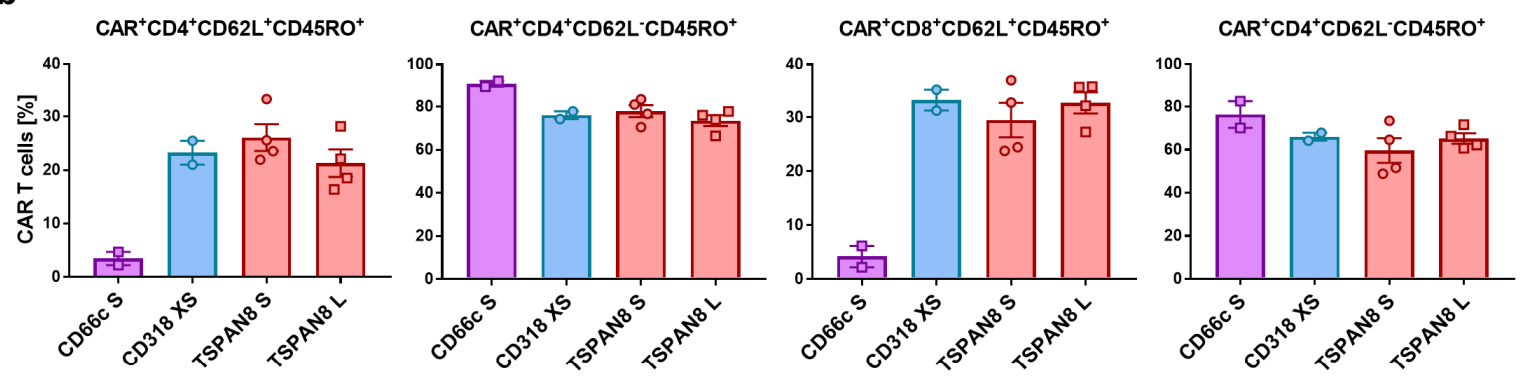

C
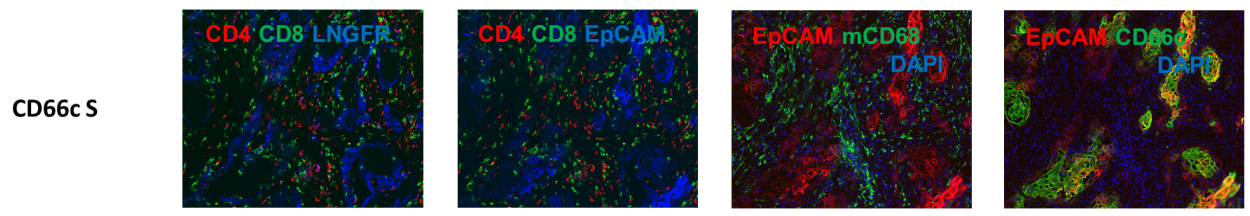

CD318 XS
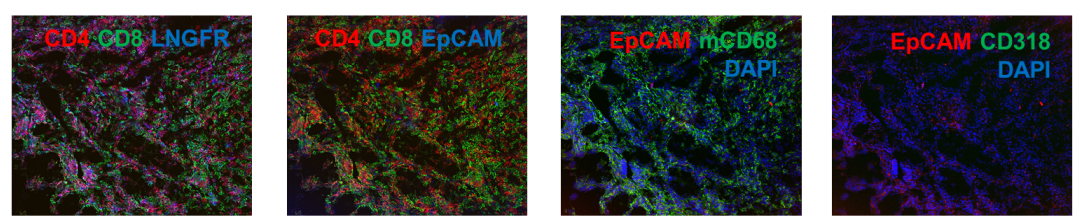

Fig. 6: Ex vivo analysis of the T cell phenotype and AsPC1 derived tumor tissues upon treatment with CAR T cells. (a) Number of CAR T cells in the spleen at the end of the experiment $25 \mathrm{~d}$ (CD66c S) and $27 \mathrm{~d}$ post CAR T cell injection (CD66c S: $n=2$, CD318 XS: $n=2$, TSPAN8 S: $n=4$, TSPAN8 L: $n=4)$ ). (b) Phenotype of CAR $\mathrm{T}$ cells in the spleen at the end of the experiment $25 \mathrm{~d}$ (CD66c S) and $27 \mathrm{~d}$ post CAR $\mathrm{T}$ cell injection, as demonstrated by the percentage of $\mathrm{T}_{\mathrm{CM}}$ and $\mathrm{T}_{\mathrm{EM}}$ ( $\mathrm{n}$ equal to a). All data are shown as mean \pm s.e.m. (c) Representative immunofluorescence images of (CAR) $\mathrm{T}$ cell tumor infiltration, macrophage tumor infiltration and target expression (CD318 XS tumor 10 days post CAR T cell injection, CD66c S tumor 25 days post CAR T cell injection).

\section{CD318 is the most promising candidate with respect to functionality and safety}

The bioinformatical analysis of CD66c, CD318 and TSPAN8 showed that all of them have restricted but detectable expression on healthy tissues (Tab. S1, Fig. S3), implying the need for an evaluation of safety concerns prior to a possible clinical translation. To overcome the inconsistency of publicly available databases we analyzed protein expression and localization by cyclic IF on 17 healthy tissues. 
Quantification of protein expression supported our initial findings that all three target candidates showed a much stronger expression on PDAC tissue as compared to all healthy tissues analyzed. CD318 showed the most favorable pattern with almost no detectable protein expression in healthy tissues. The only relevant but very low expression was restricted to the luminal side of the colon whereas T cells would most likely sense the basal or lateral membranes (Fig. S11). TSPAN8 showed a strong expression in the gastrointestinal tract, especially on colon (Fig. 7a, Fig. S12) which harbors the risk of toxicity induced by CAR T cells, whereas the weak staining observed in the medulla and skeletal muscle (Fig. S12) seemed to derive from unspecific binding as the subtraction of the background signal resulted in a negative value (Fig. 7a). In case of CD66c, some expression was observed in the hair follicle (Fig. S13) which might display a tolerable risk similar to expression detected in the ovary but limited to non-cellular mucus regions. A weak expression was present in lung tissue but much lower as compared to PDAC tissue (Fig. 7a) which might allow for a therapeutic window.

In addition, we measured expression of the three candidates on blood cells from healthy donors by flow cytometry as hematopoietic tissue displays a particular risk factor due to the presence of numerous putative target expressing cells in the preferred niche of CAR T cells. Flow cytometry based analyses showed expression of CD66c but not CD318 or TSPAN8 on several hematopoietic lineages (Fig. 7b). Whereas only minor subpopulations where CD66c-positive, the myeloid lineage, neutrophils in particular, showed a strong expression. In conclusion, CD318 is the most promising candidate for a future clinical translation in terms of predicted safety and efficacy, while in the case of TSPAN8 and CD66c safety concerns arose with respect to gastrointestinal and hematopoietic tissues, respectively. 

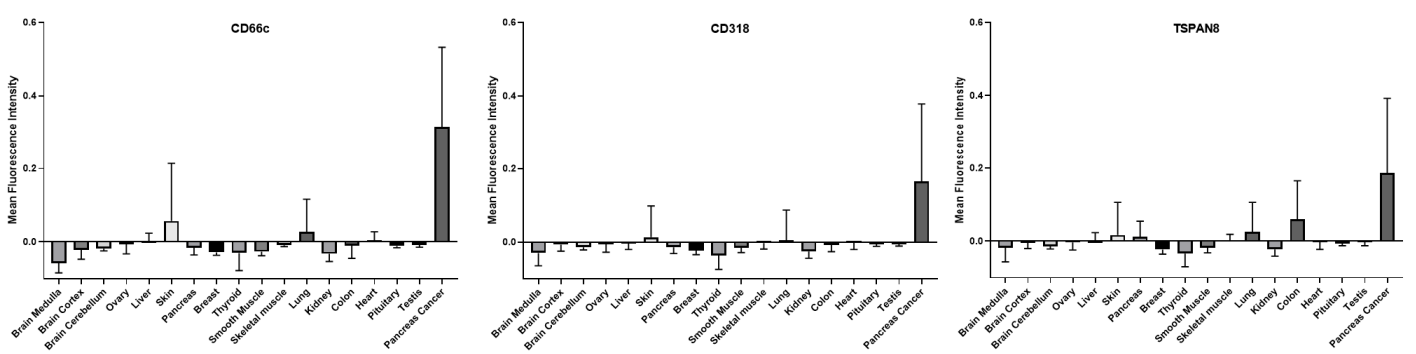

b
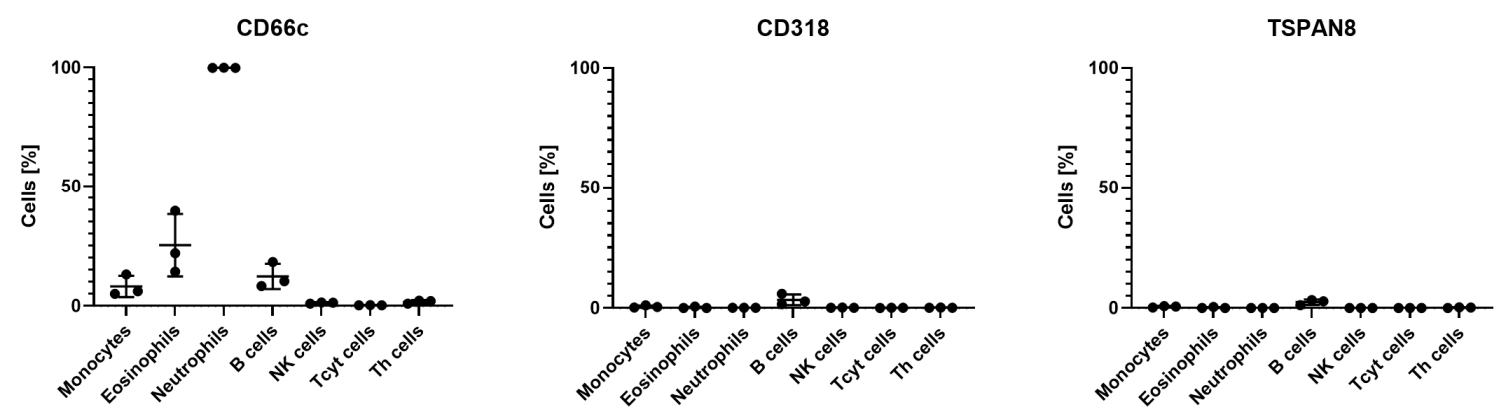

Fig. 7: Off-tumor target expression and co-culture of CAR T cells and healthy cells. (a) Quantification of target expression within different healthy tissues. Depicted is the background corrected mean fluorescence intensity of the respective tissues. Data represent mean \pm SD. (b) Flow cytometric analysis of target expression on lysed blood samples. Data represent mean \pm SD of three donors.

\section{Discussion}

In the present study, we performed an empirical screening with the aim to identify novel CAR target candidates for the treatment of PDAC, identifying four novel target candidates: CLA, TSPAN8, CD66c and CD318. We were the first discovering CLA to be expressed on epithelial derived tumors. Until now, CLA was only known to be a unique skin-homing receptor, expressed on subsets of T cells, B cells, NK cells, Langerhans cells, monocytes and dendritic cells $(14,16-19)$. It is a specialized glycosylated form of SELPLG and plays a role in tissue infiltration of immune cells through binding to E-, L- and P-selectins (25). Since it is only expressed on subsets of these cell types, we hypothesized that it might be a suitable CAR T cell target with acceptable off-tumor toxicities. However, as its expression was upregulated on activated $\mathrm{T}$ cells it posed a technical roadblock producing functional CAR T cells. CLA specific CAR T cells performed much worse underlining the intrinsic problem of CAR T cells specific for self-antigens. Other research groups developed potential solutions to this problem. While some suggested a CRISPR/Cas mediated knock-out of the antigen within the T cells $(26,27)$ others advocate to trap self-antigens in the T cells, using an ER retention domain coupled with an $\mathrm{scFv}$ specific for the self-antigen (28). 
The other identified target candidates CD66c, CD318 and TSPAN8 have been described to be overexpressed in several cancer entities (29-36). With the combination of our antibody driven screenings and the comprehensive bioinformatical and immunofluorescence analysis we could add the crucial knowledge that the expression of these target candidates is not only highly enriched in PDAC tumor cells but off-tumor expression within the human body is restricted as well. A strong support for the validity of this approach is that we identified targets which are under investigation for CAR T cell based treatment already, such as CEACAM5 (CEA) or PROM1 (CD133)(8).

We evaluated $32 \mathrm{CAR}$ constructs differing in $\mathrm{scFv}$ orientation and spacer length in vitro. While in the case of CD318 and CD66c the shorter spacer versions showed highest functionality, TSPAN8 specific constructs based on longer spacers performed better. CD318 has the longest extracellular amino acid chain (638), followed by CD66c (320) and TSPAN8 (24 and 96). Despite lacking knowledge on the exact epitope of the scFvs and the 3D structure of the extracellular domains, these findings nicely align with previous investigations pointing out that longer spacers are superior for membrane proximal epitopes and vice versa $(37,38)$.

Finally, we could show that our four best performing constructs in vitro also exhibited antitumor efficacy in two independent preclinical studies. The CD318 XS $\mathrm{V}_{\mathrm{h}}-\mathrm{V}_{1}$ and the TSPAN8 $\mathrm{S} \mathrm{V}_{\mathrm{h}}-\mathrm{V}_{1} \mathrm{CARs}$ turned out to be very promising candidates for further pre-clinical and possibly clinical evaluation based on their high efficacy. The CD66c S Vl-Vh CAR was lagging behind its in vitro efficacy but could still induce disease stabilization in one of the two xenograft models. We could show that the decreased activity of the CD66c specific CAR T cells was not caused by target downregulation and that the CAR T cell count in the spleen was indifferent to other treatment groups, therefore excluding persistence as an issue. At the tumor site, we found decreased macrophage and CAR T cell infiltration, suggesting that infiltration and possibly activation of the CAR T cells may be impaired. Other studies have shown that tonic signaling can cause such differences among in vitro and in vivo studies (39). However, we did not observe background activation of CAR T cells co-cultured with target-negative cells. Further studies are needed to elaborate on how to functionally improve this CAR or evaluate further constructs specific for CD66c which might show better functionality in vivo.

With respect to a possible clinical translation, we could corroborate the overlapping expression of CD66c and CEA, making it an interesting target candidate. In fact, it has been shown that CD66c is even stronger over-expressed in malignant tissues than CEA (40). This finding might help to manufacture fine-tuned CARs that can differentiate among differential expression levels 
on tumor and healthy cells, as shown by Caruso et al. for the target EGFR (41). However, the expression level of CD66c on neutrophils and other myeloid lineages, underlines a strong risk for inducing a severe cytokine-release-syndrome (CRS) by massive CAR T cell reactivity in the blood as observed in B-ALL patients treated with CD19 specific CAR T cells, in particular observed in patients with high tumor burden (6). However, while CRS and B cell aplasia can be treated quite well, neutropenia can only be tolerated in a very short time window rendering CD66c a high risk target, at least in a single-specificity CAR $\mathrm{T}$ cell approach (42). Combinatorial approaches using Boolean logic gating such as AND or NOT CAR constructs might offer an alternative to circumvent the expected toxicities (43-45). In this respect, also combinations of established targets such as MSN or CD133 and the novel targets described herein will broaden the repertoire to specifically target pancreatic tumor cells. Further alternatives would be the development of CARs tuned to distinguish among levels of antigen expression on tumor vs. healthy cells (41) or a tight control of time and dose of binder administration, such as anti-FITC-directed CAR-Ts or UNI-CARs $(46,47)$. In addition, spatial control of CAR expression or activation may add a further level of safety (48). These approaches have the potential to overcome on-target off-tumor toxicities as seen with single target specific CAR T cells.

CD318 and TSPAN8 have been suggested as targets for pharmacological or antibody driven therapies (49-51). While these studies show decreased tumor growth rates or disease stabilization in vitro and in vivo, our results showed complete tumor eradication, which is most likely owed to the superior cytotoxicity of T cells representing a "living drug". This has been shown in B-cell malignancies by achievement of complete disease remission upon CD19 specific CAR T cell administration after failure of prior CD19 specific antibody therapy. However, as CAR T cells are more efficient than antibodies and their pharmacokinetics hard to predict and control, they have to be administered cautiously for potential off-tumor toxicities. Our analyses aiming at predicting tissues at risk suggested that CD318 has the most favorable pattern with almost no detectable protein expression in healthy tissues. Based on our results, the highest risk for TSPAN8 specific toxicities were predicted in the gastro intestinal tract, in particular for colon, probably similar to those observed for CEA directed CARs (52). Similar to CEA, using an alternative CAR $\mathrm{T}$ cell infusion route into the hepatic artery might be a solution to overcome this possible health threat (53). In addition, the toxicity towards colon tissue could potentially be further reduced using an AND CAR strategy, with CAR T cells only being activated, when they face both CD318 and TSPAN8. 
In summary, this study is the first example combining an empiric antibody based flow cytometry screen with a novel cyclic immunofluorescence imaging platform and a comprehensive bioinformatical and experimental evaluation for off-tumor expression to identify PDAC specific cell surface markers. We identified four novel target candidates, CLA, CD66c, CD318 and TSPAN8, for a possible cellular immunotherapy of PDAC by CAR T cells. CARs specific for CD66c, CD318 and TSPAN8 showed functionality in vitro and in vivo, with CD318 being the most favorable candidate for a clinical translation.

\section{Materials and Methods}

\section{Antibody screening on patient derived xenografts}

All pancreatic ductal adenocarcinoma (PDAC) patient derived xenografts (PDX) were obtained from Charles River Discovery Research Services Germany GmbH. PDX models were dissociated using the Tumor Dissociation Kit, human in combination with the gentleMACS ${ }^{\text {тм }}$ Octo Dissociator with Heaters (both Miltenyi Biotec). Subsequently, mouse cells were depleted using the Mouse Cell Depletion Kit (Miltenyi Biotec). Resulting cell suspensions were analyzed using the MACS ${ }^{\circledR}$ Marker Screen, human (Miltenyi Biotec) a monoclonal antibody panel containing 371 pre-titrated antibodies with 9 isotype controls, or candidate antibodies selected from this panel for subsequent screening steps (Fig. S1). All samples were measured on a flow cytometer.

\section{Bioinformatical data mining and ranking}

After assigning gene symbols to the 50 top PDX-based target candidates, the corresponding gene and protein expression data were retrieved from the following data sources: Human Protein Atlas (20) (v15, https://www.proteinatlas.org/), ProteomicsDB (https://www.proteomicsdb.org/), human proteome map (http://www.humanproteomemap.org/), Genotype-Tissue Expression (GTEx, https://gtexportal.org/) and genevestigator (54).

\section{Human Protein Atlas (HPA) - antibody-based}

Protein expression scores in HPA are based on immunohistochemical data manually scored with regard to staining intensity and fraction of stained cells. We determined the fraction of the 86 cell and tissue types listed in HPA assigned to the protein expression levels "not detected", "low/medium", and "high". The top 50 target candidates were sorted based on the fraction of 
"not detected" cell types in descending order. Within a group of candidates exhibiting the same fraction of not detectable cell types, candidates were further sorted based on the fraction of "medium/high" cell types in ascending order.

\section{Human Protein Atlas (HPA) - RNAseq}

At the time of data download, gene expression scores in HPA were based on FPKM values. We determined the fraction of the 32 tissue types listed in HPA assigned to the gene expression levels "not detected" (<1fpkm), "low/medium" (1-50fpkm), and "high" (>50fpkm). The top 50 target candidates were sorted based on the fraction of "not detected" cell types in descending order. Within a group of candidates exhibiting the same fraction of not detectable cell types, candidates were further sorted based on the fraction of "medium/high" cell types in ascending order.

\section{ProteomicsDB (PDB) - Mass spectrometry-based}

Protein expression data were retrieved as $\log 10$ normalized iBAQ intensity values. We determined the fraction of the 66 tissue types listed in ProteomicsDB with missing protein expression values for the top 50 target candidates and ranked them in descending order. Only peptides unique to the target protein were considered.

\section{Human Proteome Map (HPM) - Mass spectrometry-based}

Protein expression data were retrieved as intensity levels scaled from 1 (lowest) to 10 (highest). We determined the fraction of the 17 adult tissue types and 6 cell types listed in HPM assigned to the arbitrarily defined protein expression levels "not detected/low" (levels 0-3), "medium" (4-7), and "high" (8-10). The top 50 target candidates were sorted based on the fraction of "not detected" tissues in descending order. Within a group of candidates exhibiting the same fraction of not detectable cell types, candidates were further sorted based on the fraction of "medium/high" cell types in ascending order. Only peptides unique to the target protein were considered.

\section{GTEx - RNAseq}

The data used for the analyses described in this manuscript were obtained from GTEx_Analysis_2016-01-15_v7_RNASeQCv1.1.8. We determined the fraction of the 30 tissue types listed in GTEx assigned to the median gene expression levels "not detected" (<1tpm), "low/medium" (1-50tpm), and "high" (>50tpm). The top 50 target candidates were sorted based on the fraction of "not detected" tissues in descending order. Within a group of 
candidates exhibiting the same fraction of not detectable cell types, candidates were further sorted based on the fraction of "medium/high" cell types in ascending order.

\section{Genevestigator}

Gene expression data ( $\log 2$ space) were extracted from the Genevestigator collection of microarray data (species: Homo sapiens, platform: Affymetrix Human Genome U133 Plus 2.0, sample status: healthy; root: cell type. Hierarchical categories with identical expression values were condensed). Two-dimensional hierarchical clustering was performed using $\mathrm{MeV}$ 4.9.0 (http://mev.tm4.org, distance metric: Euclidean distance, linkage method: complete linkage clustering; leaf order optimization: genes and samples).

\section{Rank sum}

The final rank was calculated based on the quotient of the rank sum from all data sources divided by the number of data sources it was found in.

\section{Ranking according to Perna et al. (2017)}

Perna and colleagues (23) developed an algorithm to identify expression of target candidates throughout the human body using HPA, PDB and HPM as input databases. We recreated their method using publicly available data sets and a few methodological differences. We also $\log 10$ transformed the data from HPM and PDB but did not perform a temporarily correction of HPM data for multiple gene assignments. Subsequently, normal distributions were fitted to the data using the Levenberg-Marquardt method. For some data sets this required ignoring data close to zero which we assume is noise introduced by various effects (for instance genes never expressed in certain tissue types) and which did not fit in with a normal distribution. This curve fitting produced the average and standard distribution values we used and peak maximum and standard deviation were calculated for each curve.

Data were then binned as follows: Expression values between the maximum peak and one standard deviation above were considered "medium" (2). Values above this threshold were considered to be "high" (3) expressed. Expression values between the peak maximum and the standard deviation below were considered "low" (1) and all values below this threshold were categorized as "not detected" (0).

As the tissue names differed between the different data bases, they were harmonized to a consensus tissue name as suggested by Perna et al. (Tab S1). The final table was then created in depicting the highest value for the respective tissue and target candidate from all data bases. 


\section{Cyclic immunofluorescence staining}

Primary or PDX derived PDAC specimens and healthy tissues were embedded in Tissue Freezing Medium (Leica) and stored at $-70^{\circ} \mathrm{C}$ until further use. Afterwards, three $8 \mu \mathrm{m}$ sections were cut on a CM3050 S cryostat (Leica), collected on SuperFrost ${ }^{\circledR}$ Plus slides (Menzel) and stored no longer than 2 weeks at $-70^{\circ} \mathrm{C}$. On the day of use, sections were thawed in $-20^{\circ} \mathrm{C}$ acetone and then processed for hematoxylin and eosin (HE) or immunofluorescence (IF) staining.

For HE staining the acetone fixed section was dipped thrice in water and then incubated in Meyer's hematoxylin solution (Carl Roth) for $7 \mathrm{~min}$. The section was transferred for $5 \mathrm{~min}$ into water and subsequently shortly dipped into $\mathrm{HCl} / \mathrm{ethanol}$ solution $(1 \% \mathrm{HCl}, 70 \%$ ethanol). Afterwards, it was rinsed with water and stained in Eosin/ethanol solution ( $0.5 \mathrm{~g}$ Eosin $\mathrm{Y}$ in $100 \mathrm{ml} \mathrm{70 \%} \mathrm{ethanol)} \mathrm{for} 5 \mathrm{~min}$. The section was then dehydrated stepwise first in 70\% ethanol, then $96 \%$ ethanol and finally pure ethanol with 3 dips for each concentration. Following this, the tissue was cleared in two changes xylene (Carl Roth) with three dips each. The HE stained section was covered in Roti®-Histo Kit (Carl Roth) and cover slipped. Subsequently the section was examined using a light microscope to define a region of interest, which was confirmed by a pathologist to be a neoplastic region.

After definition of a region of interest, another $8 \mu \mathrm{m}$ section of the same specimen was thawed in $-20^{\circ} \mathrm{C}$ acetone. The fixed tissue was stored shortly in autoMACS ${ }^{\text {тм }}$ Running Buffer (Miltenyi Biotec) and introduced into a MACSima ${ }^{\mathrm{TM}}$ prototype. MACSima ${ }^{\mathrm{TM}}$ is a novel cyclic IF imaging platform enabling fully automated IF imaging of individual biological samples. The system operates by iterative fluorescent staining, image acquisition, and signal erasure, using multiple fluorochrome-labeled antibodies per cycle (manuscript under preparation). Images that were generated using MACSima ${ }^{\text {тм }}$ were overlaid and analyzed using ImageJ $1.49 \mathrm{v}$.

\section{Healthy tissue microarray analysis and quantification of expression}

HDR images were checked manually using Image J $1.49 \mathrm{v}$ for any structures that could disturb the subsequent automated analysis. Therefore, images containing clumped conjugates, swollen nuclei, fabric remnants or other artefacts were excluded from further analysis. All other images were uploaded into the CellProfiler v2.2.0 software (55). We defined cell nuclei by DAPI staining as primary objects and excluded nuclei touching the image border. Secondary objects were defined as primary objects expanded by 5-6 pixels depending on the nuclei density, allowing us to approximate the shape of a cell. We then extracted the mean fluorescence 
intensities of the respective target candidate for each cell (secondary object). In order to define a background, we used the unspecific REA control (S) antibody as an isotype control and subtracted the control intensity from the target candidate intensity. For image display in the figures, we uploaded the respective PDAC image of each target to ImageJ and auto adjusted it. We then uploaded all other tissue images of the same excitation time and adjusted the display to the same parameters as for the PDAC image.

\section{Flow cytometric analysis of primary PDAC specimen}

The tumors were dissociated using the Tumor Dissociation Kit, human in combination with the gentleMACS $^{\mathrm{TM}}$ Octo Dissociator with Heaters (both Miltenyi Biotec) and prepared for flow cytometry as described above.

\section{Cell lines and culture conditions}

Human embryonic kidney 293T (HEK293T), BxPC3 and AsPC1 cells were obtained from ATCC and cultured as recommended. For co-culture with T cells they were transduced to express firefly luciferase (Luc) and green fluorescent protein (GFP). The PanCa0201 cell line was derived from a human primary PDAC biopsy, dissociated as described above and tumor cells isolated prior to seeding using the Tumor Cell Isolation Kit, human (Miltenyi Biotec). Subsequently tumor cells were cultivated using the Pancreas TumorMACS Medium (Miltenyi Biotec). The PanCa0201 cell line was transduced as well to express Luc and GFP. To validate authenticity of the cell lines used, we used the Human STR Profiling Cell Authentication Service (ATCC).

\section{Isolation of $T$ cells and generation of CAR T cells}

Peripheral blood mononuclear cells (PBMCs) were isolated by density gradient centrifugation from buffy coats of healthy anonymous donors (German Red Cross Dortmund). $\mathrm{T}$ cells were purified from PBMCs using the Pan T Cell Isolation Kit, human (Miltenyi Biotec) and activated in TexMACS ${ }^{\text {тм }}$ Medium (Miltenyi Biotec) containing T Cell TransAct ${ }^{\mathrm{TM}}$, human (Miltenyi Biotec) and $100 \mathrm{IU} / \mathrm{ml}$ of recombinant Human IL-2 IS, research grade (IL-2) (Miltenyi Biotec) or $12.5 \mathrm{ng} / \mathrm{mL}$ of recombinant human interleukin IL-7 and $12.5 \mathrm{ng} / \mathrm{mL}$ of recombinant human IL-15 (both Miltenyi Biotec) for the CLA expression experiments. T cells were transduced 24 $\mathrm{h}$ after activation using vesicular stomatitis virus glycoprotein G (VSV-G) pseudotyped lentiviral supernatants derived from transfected HEK293T cells. Supernatants were concentrated and stored at $-70^{\circ} \mathrm{C}$ until transduction. $3 \mathrm{~d}$ post activation, T Cell TransAct ${ }^{\mathrm{TM}}$, human was washed out of the medium and T cells were cultured with $100 \mathrm{IU} / \mathrm{ml} \mathrm{IL}-2$ containing 
TexMACS ${ }^{\mathrm{TM}}$ Medium. T cells were used for in vitro assays directly or frozen until further use for in vivo testing 12-14 d after purification from PBMCs. Frozen T cells that were used for in vivo testing were thawed $24 \mathrm{~h}$ before injection in TexMACS ${ }^{\mathrm{TM}}$ Medium without further supplements. On the day of use, the amount of living CAR T cells was determined using flow cytometry and staining T cells with 7-AAD and anti-human LNGFR (both Miltenyi Biotec).

\section{Generation of CAR plasmids}

Plasmids encoding the CAR constructs were prepared using standard molecular biology and cloning techniques. They all comprised murine single-chain variable fragments (scFvs) specific for the respective target candidates (CLA, CD66c, CD318 or TSPAN8) that were preceded by a CD8 $\alpha$ leader peptide. Sequences for the light and heavy chain of the scFvs were the same, as for the antibodies used during target discovery. scFvs were used in both possible orientations and connected with a glycine-serine linker. scFvs were followed by spacers differing in size. The library of backbones comprised either a human IgG4 hinge from the $\mathrm{CH} 2-\mathrm{CH} 3$ domains (L; long spacer; 228 aa), a human IgG4 hinge from the CH3 domain (medium spacer; 119 aa), a human IgG4 hinge from the sequence between $\mathrm{CH} 2$ and $\mathrm{CH} 1$ domain (XS; extra short spacer; 12 aa) or a human CD8 $\alpha$ spacer (S; short spacer; 45 aa). All IgG4 spacer domains contained a 4/2 NQ mutation in the $\mathrm{CH} 2$ domain as well as a $\mathrm{S} \rightarrow \mathrm{P}$ substitution in the hinge region in order to reduce FcR binding All CARs shared the same CD8 $\alpha$ transmembrane domain and featured $4-1 \mathrm{BB} / \mathrm{CD} 3 \zeta$ derived intracellular signaling domains. The CAR sequence was linked to a P2A sequence to induce co-expression of truncated low-affinity nerve growth factor receptor (LNGFR).

\section{Target-T cell co-culture assays}

Target cells were inoculated in duplicates in 96-well culture plates at densities of $1 \times 10^{4}-5 \times 10^{4}$ cells per well in $100 \mu \mathrm{l}$ of their respective culture medium. Directly thereafter or $24 \mathrm{~h}$ later $\mathrm{CAR}^{+} \mathrm{T}$ cells were added in $\mathrm{E}: \mathrm{T}$ ratios of either $5: 1,2: 1$, or $1: 1$. Final vessel volume equaled $200 \mu \mathrm{l}$. The amount of T cells in the Mock control was adjusted to the number of total T cells in the CAR group with the highest total cell count. Cytotoxicity was measured as a decrease in green surface area with an IncuCyte ${ }^{\circledR}$ S3 Live-Cell Analysis System (Sartorius). The surface area at the start of the experiment was considered $100 \%$ and the following decrease or increase in surface area was set into relation. At the end of co-culture, either after $48 \mathrm{~h}$ or $6 \mathrm{~d}$, CAR T cells were analyzed with a flow cytometer for their expression of TIM-3 or 4-1BB, LAG-3, and PD-1. In addition, after $48 \mathrm{~h} 100 \mu \mathrm{l}$ of medium were used for analysis of the cytokine release. 
In case co-culture continued, $100 \mu \mathrm{l}$ of fresh target cell medium were added again. Cytokine content was measured with the MACSPlex Cytokine 12 Kit, human (Miltenyi Biotec).

\section{Normalization for in vitro killing, cytokine release and marker expression for cross- comparability}

To better compare the effectivity of the CAR constructs between cell lines, assays and donors, we normalized the readouts for each assay. We normalized the cytokine release of GM-CSF, IL-2, TNF- $\alpha$ and INF- $\gamma$ to the highest value for each cytokine and calculated from them an average resulting in the cytokines value. We normalized to the highest number of $\mathrm{CAR}^{+}$cells expressing the respective marker, with the markers being PD-1, LAG-3, TIM-3, 4-1BB and 41BB/LAG-3 double positive. We calculated then the average of the normalized values resulting in the marker value. For killing we used the highest decrease after $48 \mathrm{~h}$ and $6 \mathrm{~d}$ in green surface area as value to normalize to. Again the average of $48 \mathrm{~h}$ and $6 \mathrm{~d}$ values resulted in the killing value. Balloon diagrams were created using R (v3.4) with the packages reshape2, ggplot2 and viridis.

\section{Preclinical mouse models}

All experiments were approved by the Governmental Review Committee on Animal Care in NRW, Germany and performed according to guidelines and regulations (Landesamt für Natur, Umwelt and Verbraucherschutz NRW, Approval number 84-02.04.2017.A320). Cell line derived xenografts were established by injecting $1 \times 10^{6} \mathrm{BxPC} 3$ or AsPC1 cells subcutaneously in the right flank of NOD SCID gamma (NSG; NOD.Cg-PrkdcscidIl2rgtm1Wjl/SzJ) mice (Jackson Laboratory, Bar Harbor, USA, provided by Charles River). Once tumors reached 25 $\mathrm{mm}^{2}$ as measured with a caliper, $5 \times 10^{6}$ or $1 \times 10^{7}$ CAR T cells were injected into the tail vein. The amount of injected Mock T cells was adjusted to the number of total T cells in the CAR group with the highest total cell count. Anti-tumor response was measured longitudinally using an in vivo imaging system (PerkinElmer, Waltham, USA) after intraperitoneal injection of 100 $\mu \mathrm{L}$ (30 mg/mL) D-Luciferin (Potassium Salt, LUCK-2G, GoldBio). All measures to secure the wellbeing of mice were executed following the relevant animal use guidelines and ethical regulations. Where possible, tumors were excised and cut in two halves. One half was embedded in Tissue Freezing Solution (Leica) and stored at $-70^{\circ} \mathrm{C}$ until further use. The other half was dissociated as stated above and T cells were analyzed using flow cytometry. Ex vivo analysis of the spleen was performed after dissociation using the gentleMACS ${ }^{\text {тм }}$ Octo Dissociator with Heaters according to the manufacturers protocol (Miltenyi Biotec) and red blood cell lysis using Red Blood Cell Lysis Solution (Miltenyi Biotec) using a flow cytometer. 


\section{Flow cytometry}

All samples were measured on a MACSQuant ${ }^{\circledR}$ Analyzer 10 and analyzed using the MACSQuantify ${ }^{\text {TM }}$ Software. Antibody conjugates that were used for surface marker expression on PDAC primary tissues, PDXs and T cell analysis were the same as in the MACS Marker Screen. Only conjugated fluorophores were exchanged for some instances. From Thermo Fisher: Chicken anti-Goat IgG $(\mathrm{H}+\mathrm{L})$ Cross-Adsorbed Secondary Antibody, Alexa Fluor 647. From Merck: Anti-Mouse IgG (Fab specific) antibody produced in goat.

Stainings conducted with antibody conjugates from Miltenyi Biotec. were performed as recommended by the supplier. Therefore, conjugates were added to cell suspensions in a final ratio of $1: 11$ and incubated $10 \mathrm{~min}$ at $4{ }^{\circ} \mathrm{C}$. Antibodies from Sigma-Aldrich and Thermo Fisher were applied at concentrations of $10 \mu \mathrm{g} / \mathrm{ml}$ and incubated for $30 \mathrm{~min}$ at $4^{\circ} \mathrm{C}$. Subsequent to the incubation a washing step was performed adding PEB in an excess of 9 times the staining solution. Cells were spun down and resuspended in appropriate volumes of PEB and measured on a flow cytometer. Dead cells were excluded using PI or 7-AAD.

\section{Statistics}

Unless otherwise specified, all graphs show the mean with error bars representing the standard error of the mean. Statistical comparisons between more than two groups were conducted by Two-way ANOVA with p-value $<0.05$ using GraphPad Prism 7. For all mouse experiments, the number of independent mice used is listed in the figure legend. For cytokine, marker and in vitro cell killing experiments, $n=2$ wells, and experiments were repeated at least twice.

\section{Ethical concerns}

For all studies using human PDAC primary tissue, written informed consent was obtained following the guidelines of the approved Universitätsmedizin Göttingen Review Board protocol.

Peripheral blood mononuclear cells (PBMCs) were isolated from buffy coats of healthy anonymous volunteers that were purchased from the German Red Cross Dortmund. All blood samples were handled following the required ethical and safety procedures.

All animal experiments were approved by the Governmental Review Committee on Animal Care in NRW, Germany and performed according to guidelines and regulations.

Healthy whole blood samples were taken from voluntary healthy donors that gave their written consent before. 


\section{Acknowledgments}

We want to thank Michail Knaul and Jutta Kollet for their help and advice regarding bioinformatical matters and Sandy Reiß, Lara Minnerup, Vera Dittmer and Abigail J. Deloria for excellent technical assistance.

\section{Author contributions}

D.S. and O.H. wrote the manuscript, D.S., A.B. and O.H. designed the study, D.S. and L.N.K. conducted experiments for target discovery, D.S., G.T.H. and S.T. did the bioinformatic analysis, D.S. performed in vitro assays, D.S., W.A.R., J.H., J.B. and C.L. performed the in vivo studies, D.S. and D.L. cloned the CAR library, J.S., D.J.A. and P.S. supplied materials and resources, J.M.G, F.A., A.K., A.B., C.H., D.E. and O.H. supervised the project, all authors discussed the data and reviewed the manuscript.

\section{Competing interests}

D.S., L.N.K, S.T, G.T., D.A., W.A.R, J.H., J.B., C.L., D.L., A.K., C.H., D.E., A.B. and O.H. are employees of Miltenyi Biotec B.V. \& Co. KG.

J.S. is employee of Charles River Discovery Research Services GmbH.

There are patent applications pending related to this work.

\section{Data and materials availability}

Data are available from the corresponding author .

\section{Supplementary Materials}

Fig. S1 Target discovery workflow

Fig. S2 Gating strategy for flow cytometric target candidate analysis of dissociated patient derived xenograft tissues

Fig. S3 Bioinformatics based ranking of target candidates

Fig. S4 Gating strategy for flow cytometric target candidate analysis of dissociated primary pancreatic ductal adenocarcinoma

Fig. S5 Expression of LNGFR and CAR in HEK293T cells lipofected with the CAR constructs Fig. S6 CAR T cells specific for CLA, CD66c, CD318 and TSPAN8 exhibit no cytotoxic effects on target negative cells

Fig. S7 Evaluation of CAR T cell functionality in vitro

Figure S8 CLA is a expressed on T cells and upregulated upon activation.

Fig. S9 Mock T cells have no effect on tumor outgrowth in both in vivo models

Fig. S10 Analysis of target expression upon xenotransplantation of AsPC1 and BxPC3 cells in vivo

Fig. S11 CD318 expression on healthy tissues assessed by cyclic immunofluorescence imaging. Fig. S12 TSPAN8 expression on healthy tissues assessed by cyclic immunofluorescence imaging. 
Fig. S13 CD66c expression on healthy tissues assessed by cyclic immunofluorescence imaging. Table S1 Bioinformatics based ranking of target candidates

Table S2 Antibody list used in the cyclic immunofluorescence runs

Table S3 Raw-Data of the in vitro assays performed with all L, S and XS spacer CAR T cells Table S4 Raw-Data of the in vitro assays performed with the most efficient CAR T cells for selection of the in vivo candidates

Supplementary materials download link

Supplementary Excel files can be found here:

https://doi.org/10.25625/DIUWVB 


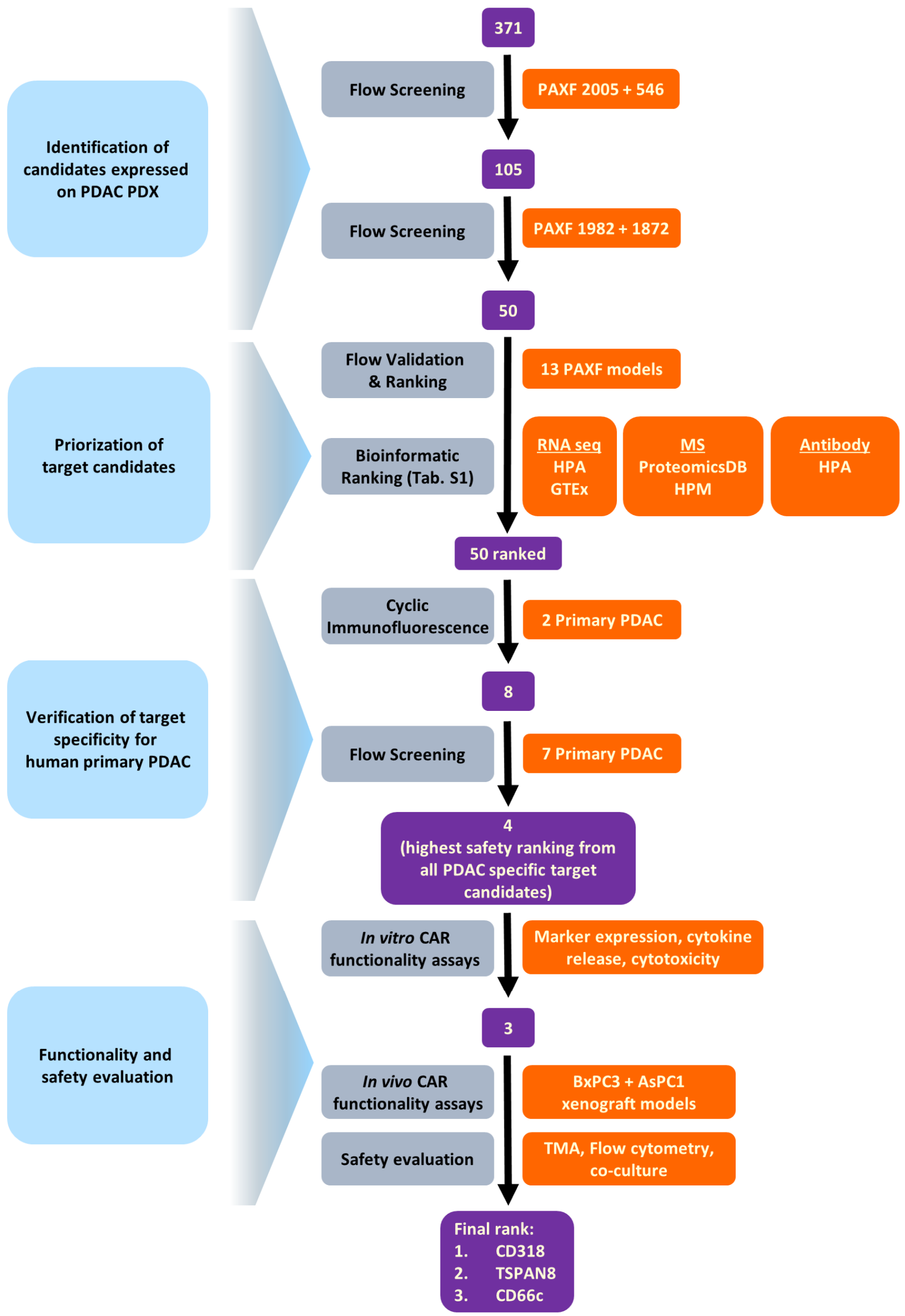

Fig. S1: Target discovery workflow. Scheme of the workflow for identification of novel target candidates as applied in this study. 
a

Debris exclusion

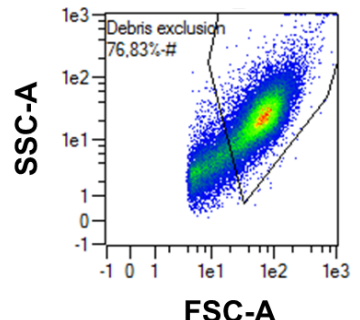

e

Sample gating

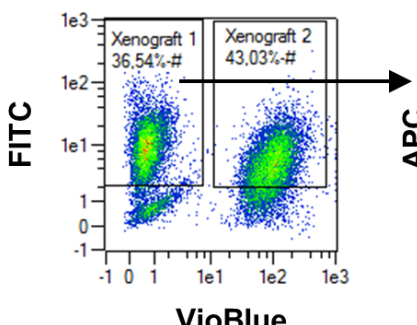

b

Dead cell exclusion

$\overline{\mathbf{\alpha}}$

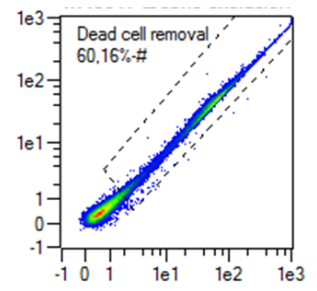

PE

f

Screening Xenograft 1
C

\section{Doublet exclusion}

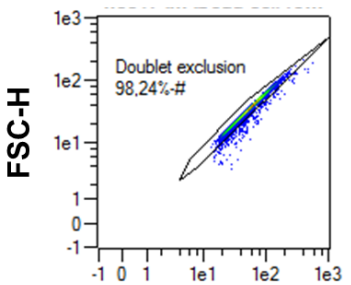

FSC-A

g

Sample gating d

Mouse cell exclusion

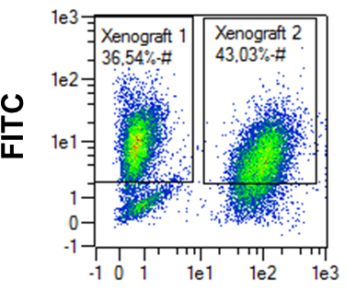

VioBlue

h

Screening Xenograft 2
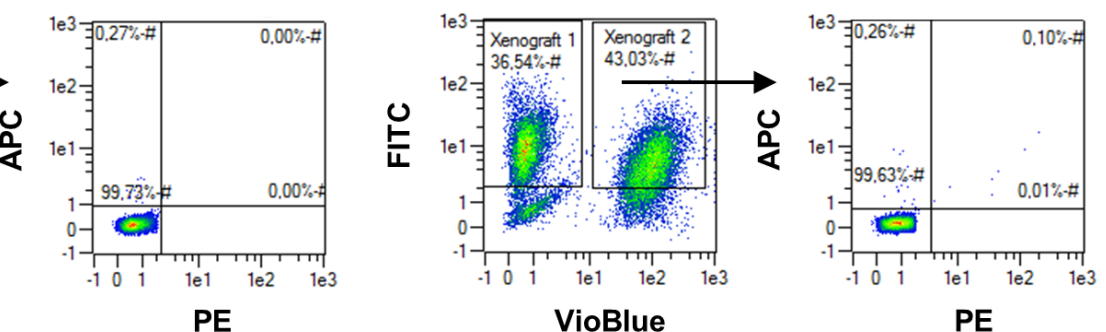

Fig. S2: Gating strategy for flow cytometric target candidate analysis of dissociated patient derived xenograft tissues. Representative flow cytometric analysis of two patient derived xenograft models. Tumor tissue was dissociated into a single cell suspension, stained with the respective antibodies and analyzed by flow cytometry. Debris was excluded by SSC-A/FSC-A gating (a) and dead cells were excluded using PI or 7-AAD (b). After exclusion of doublets (c) mouse cells were excluded by gating on hEpCAM ${ }^{+}$human tumor cells (d). One xenograft was stained before with CellTrace ${ }^{\text {тм }}$ Violet to distinguish between both samples (d). The target candidate expression was analyzed on $\mathrm{hEpCAM}^{+}$cells (e,f and g,h). 


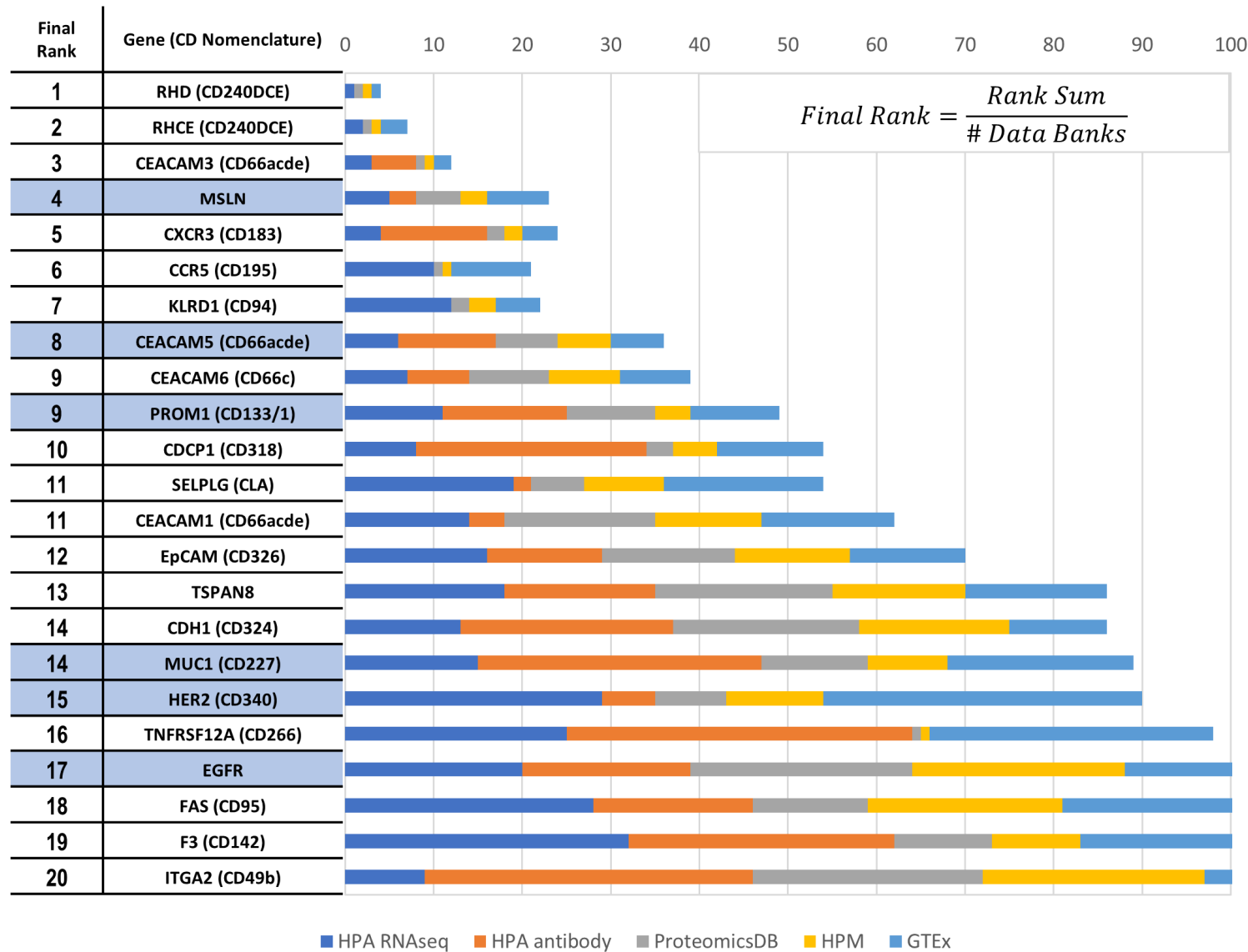

b

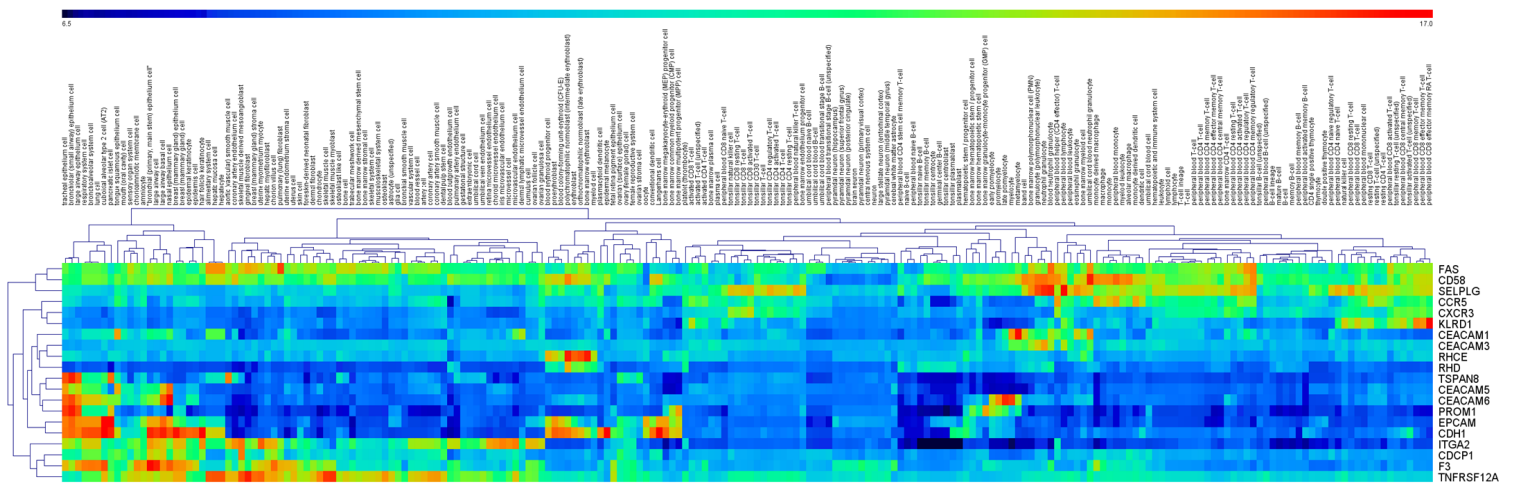

Fig. S3: Bioinformatics based ranking of target candidates. (a) 20 most promising candidate genes ranked by the quotient of the rank sum and number of data sources the respective gene was found in. The ranking also includes targets currently under clinical investigation which 50 appeared in our ranking but were not included in the initial antibody array or failed to meet inclusion criteria during the PDX screening (blue filling). Final rank with lowest off-tumor expression at the top; HPA RNAseq or antibody $=$ Human Protein Atlas mRNA or antibodybased protein expression, HPM = Human Proteome Map, GTEx = Genotype-Tissue Expression. (b) Heatmap of the expression of 20 most promising genes in healthy tissues (data source: Genevestigator). 
a

Debris exclusion

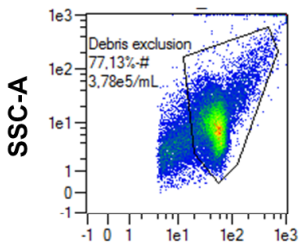

FSC-A

e

Tumor cells

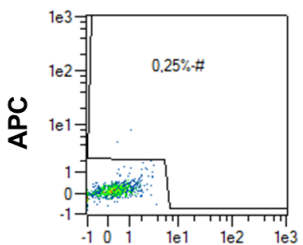

PE

h

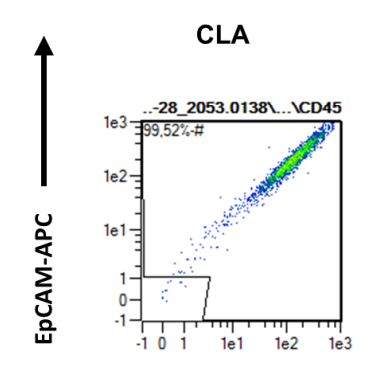

Target-PE b

Dead cell exclusion

$\bar{\alpha}$

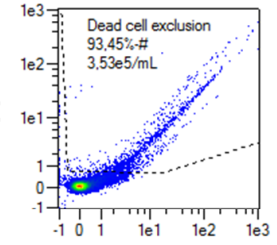

PE

f

CD45+ cells

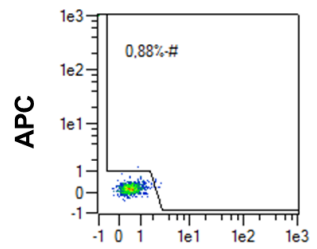

PE
Doublet exclusion

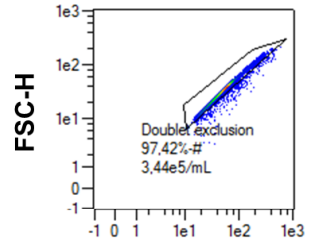

FSC-A

g

others (Double negative)

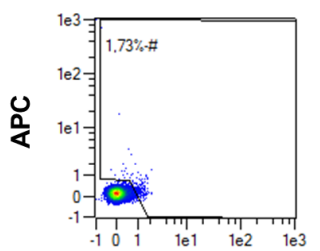

PE d

Sample gating

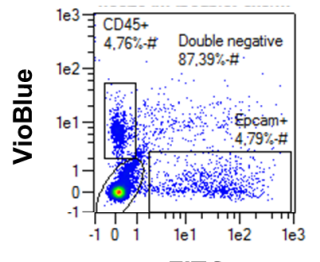

FITC

TSPAN8

CD318

CD66c
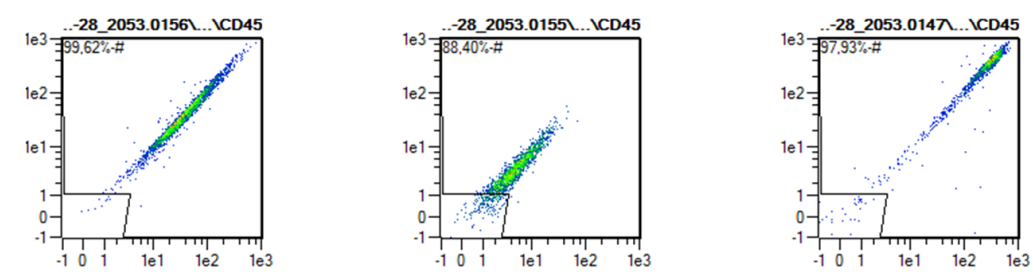

Fig. S4: Gating strategy for flow cytometric target candidate analysis of dissociated primary pancreatic ductal adenocarcinoma. Representative flow cytometric analysis of a primary pancreatic ductal adenocarcinoma. Tumor tissue was dissociated into a single cell suspension, stained with the respective antibodies and analyzed by flow cytometry. Debris (a), dead cells (b) and doublets were excluded (c). Target candidate expression was analyzed on leukocytes $\left(\mathrm{CD}_{4}{ }^{+}\right)$, tumor cells $\left(\mathrm{EpCAM}^{+}\right)$or double negative cells $(\mathrm{d}, \mathrm{e}, \mathrm{f}, \mathrm{g})$. (h) Representative density plots of CLA, TSPAN8, CD318 and CD66c expression on primary PDAC. 


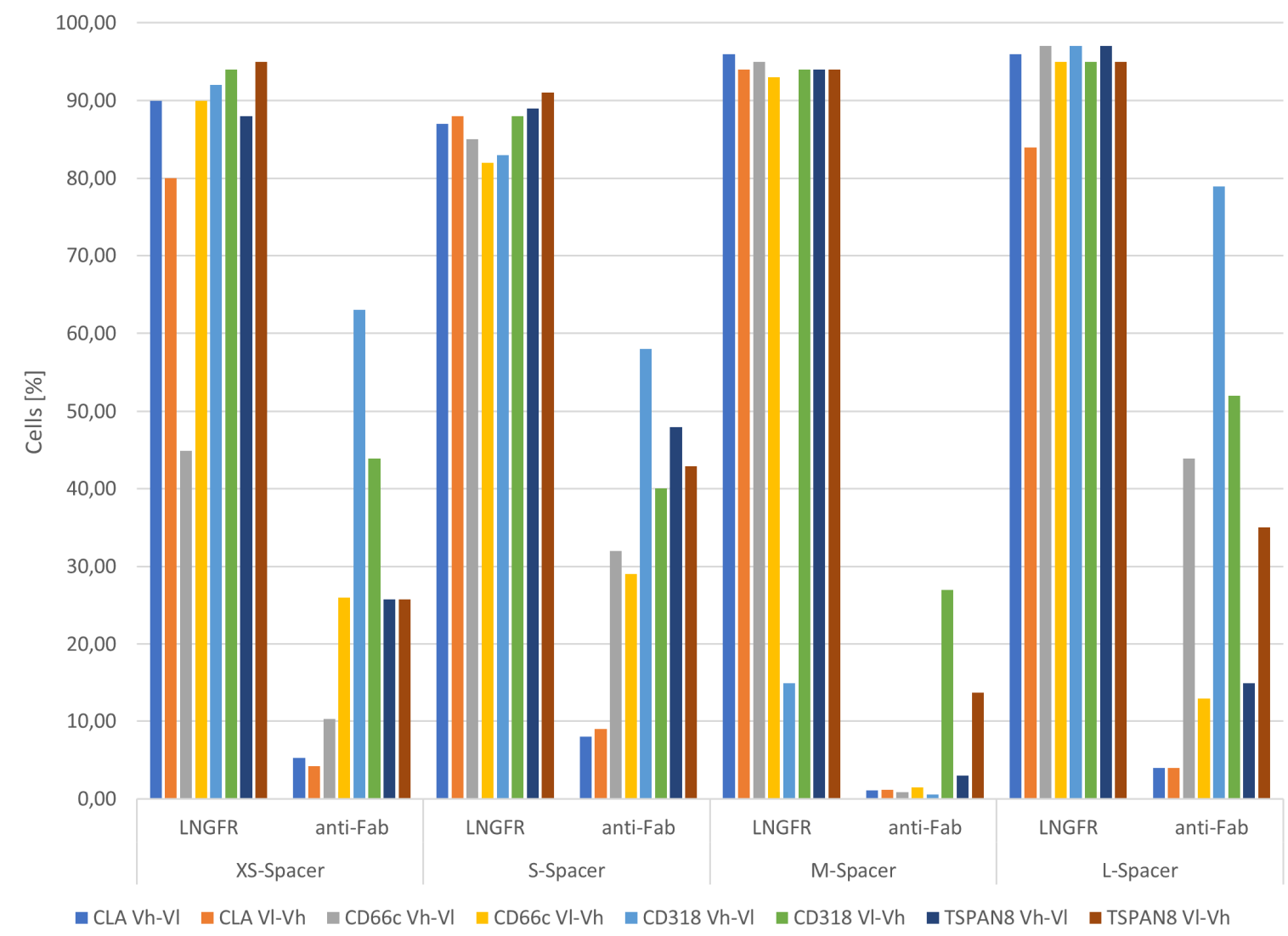

Fig. S5: Expression of LNGFR and CAR in HEK293T cells lipofected with the CAR constructs. Frequency of HEK293T cells which are expressing LNGFR and the respective CAR on the cell surface as measured by flow cytometry. HEK293T cells were lipofected with CAR plasmids to assess the general ability of the CAR cassette in the plasmid to be expressed (as measured by the reporter protein LNGFR) and if the CAR construct can be detected on the cell surface. Data are representative of two independent lipofections. 

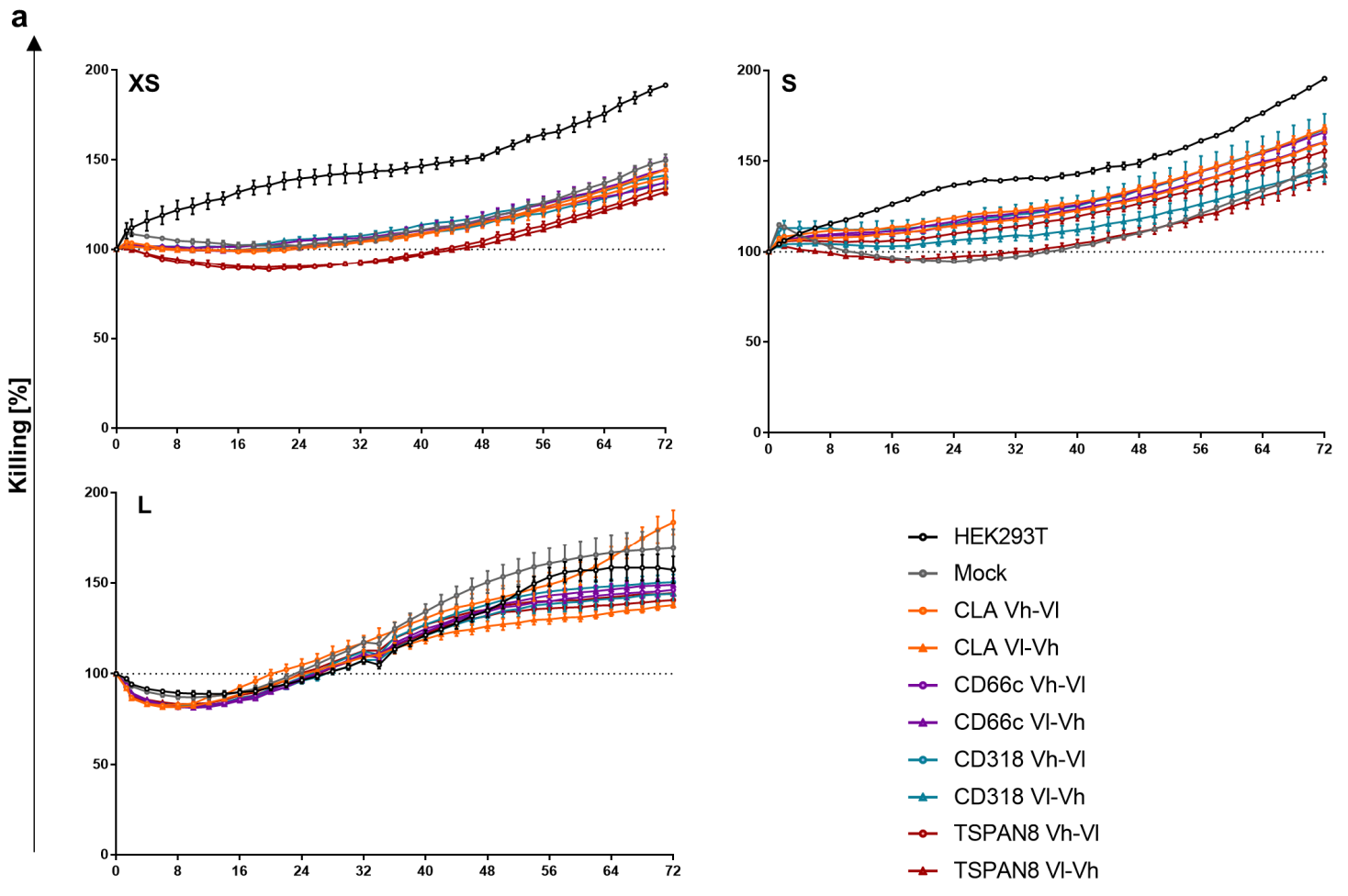

Time $[\mathrm{h}]$

b

xs

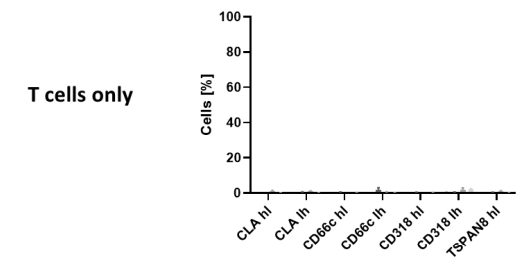

T cells stimulated

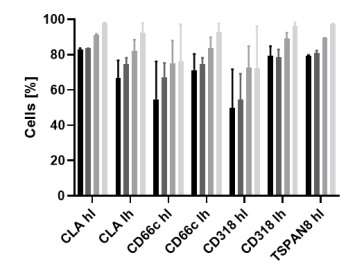

s
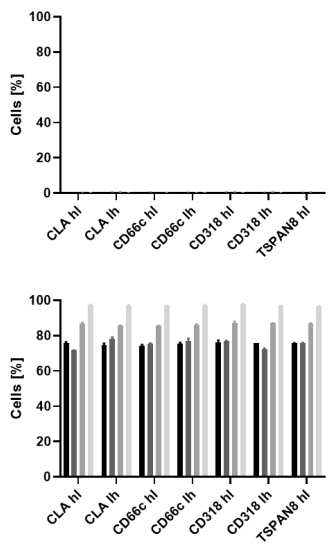

$\mathbf{L}$

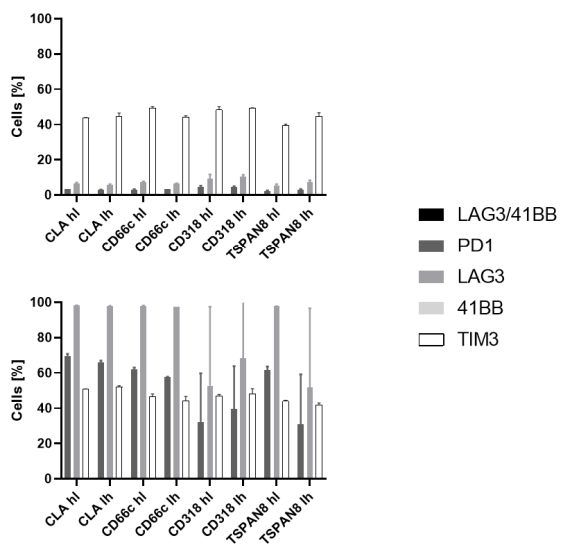

Fig. S6: CAR T cells specific for CLA, CD66c, CD318 and TSPAN8 exhibit no cytotoxic effects on target negative cells. (a) $1 \times 10^{5}$ CAR T cells were inoculated with 5x104 HEK293T cells in a 96-well cavity. The amount of Mock T cells was adjusted to the CAR group with the highest amount of total $\mathrm{T}$ cells per well. Killing was measured as percentage difference of green surface area measured automatically with an IncuCyte S3 device. Values represent mean \pm s.e.m $(n=2)$. (b) Activation marker expression upon culture without any stimulus or with PMA/Ionomycin stimulation. Shown are the means \pm s.d. $(n=2)$. Activation marker expression was measured after $48 \mathrm{~h}$. 

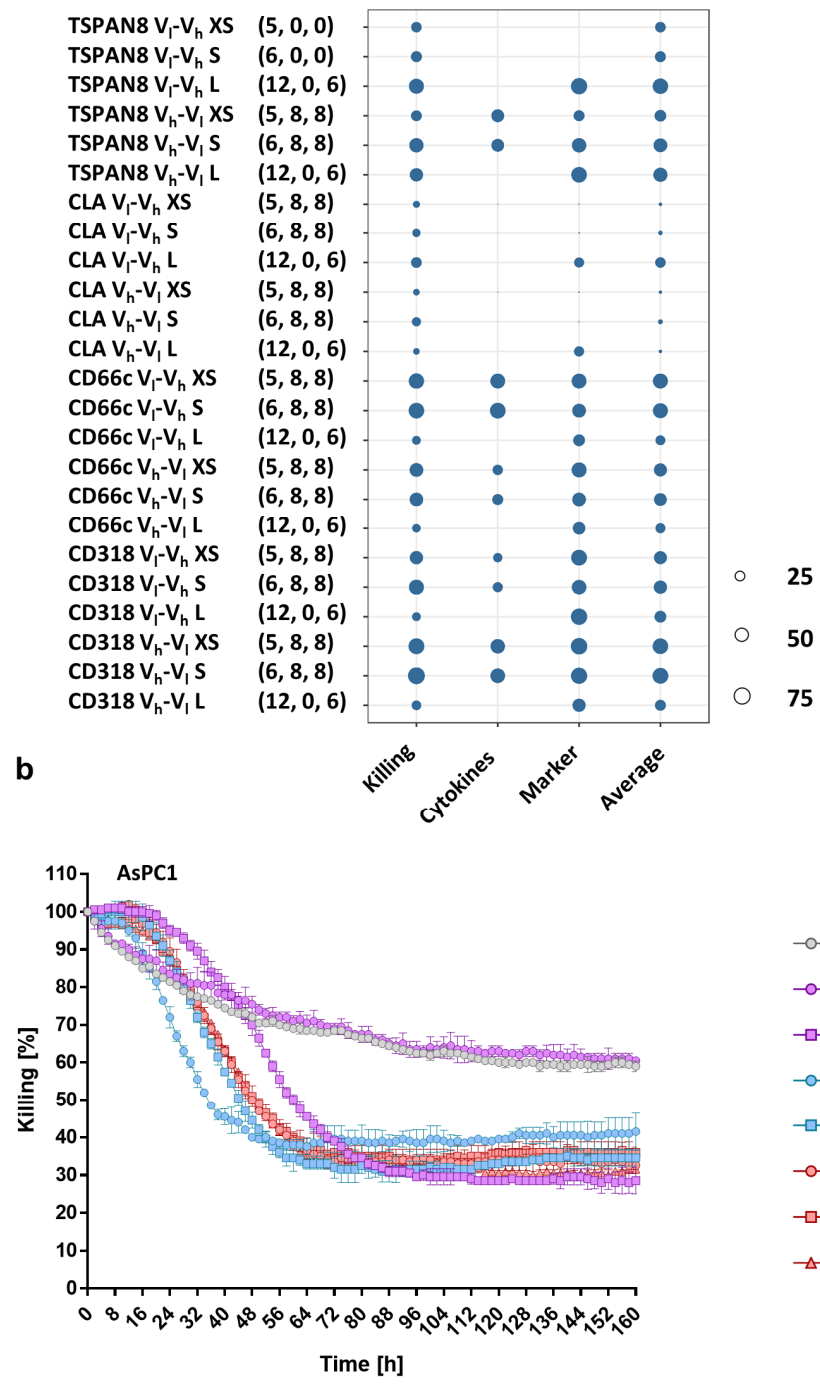

$$
\begin{aligned}
& \text {-o- Mock } \\
& \text {-O- CD66c V V - } V_{h} X S \\
& \rightarrow-\mathrm{CD}_{0} 6 \mathrm{c} \mathrm{V}_{\mathrm{l}} \mathrm{V}_{\mathrm{h}} \mathrm{S} \\
& \text {-O- CD318 } \mathrm{V}_{\mathrm{h}}-\mathrm{V}_{\mathrm{l}} \mathrm{XS} \\
& \rightarrow-C D 318 V_{h}-V_{l} S \\
& \text {-O- TSPAN8 } V_{h}-V_{1} S \\
& \text { - }- \text { TSPAN8 } \mathrm{V}_{\mathrm{h}}-\mathrm{V}_{\mathrm{l}} \mathrm{L} \\
& \triangle \text { TSPAN8 } V_{1}-V_{h} L
\end{aligned}
$$

C
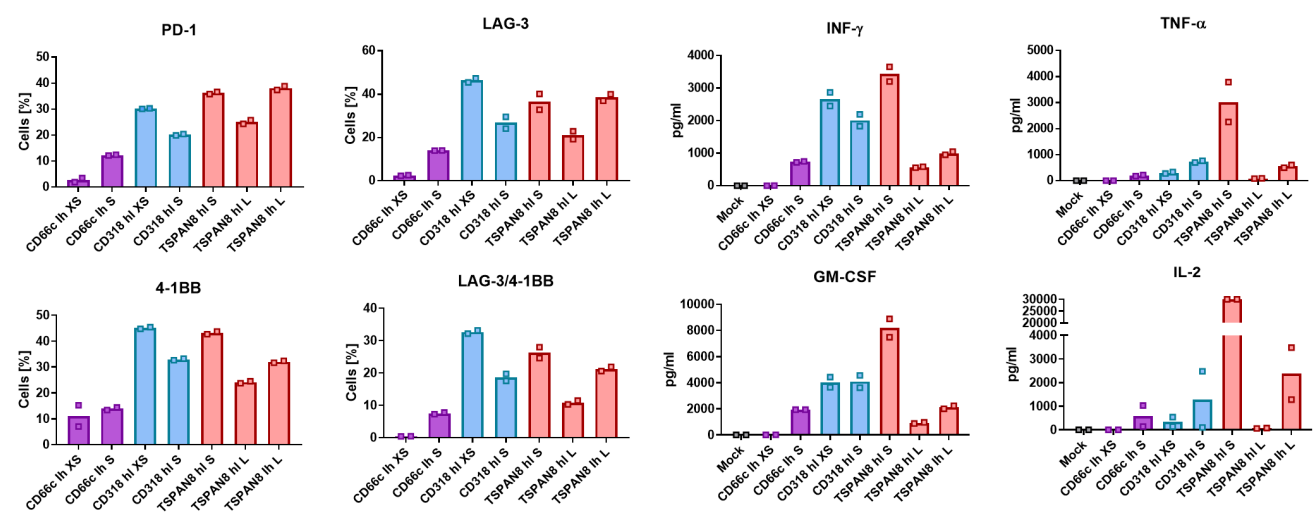

Fig. S7: Evaluation of CAR T cell functionality in vitro. (a) Results of the initial screening assays. Average relative target cell killing, cytokine release, marker upregulation and overall performance (displayed as circle size, $\mathrm{x}$ axis) for the respective CAR constructs ( $\mathrm{y}$ axis). The number of replicates is indicated in parentheses behind the construct name. (First value $=\mathrm{n}$ of killing assays, second value $=\mathrm{n}$ of cytokine release assays, third value $=\mathrm{n}$ of marker upregulation measurements). (b) Representative result for the kinetics of AsPC1 target cell killing by the best performing CAR constructs. (c) Representative results showing cytokine release and activation marker expression patterns upon co-culture with AsPC1 target cells. Shown are the means \pm s.e.m $(n=2)$. Activation marker expression was measured at end point of cytotoxicity assay, cytokine release patterns were measured after $48 \mathrm{~h}$. 


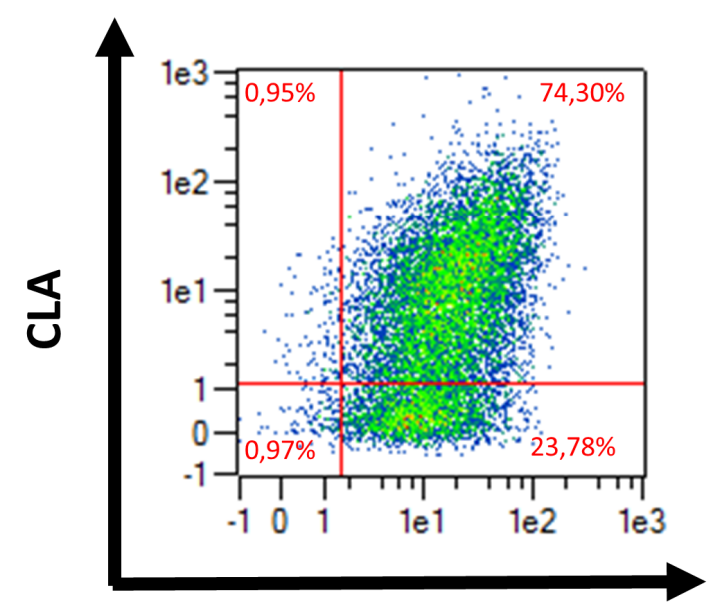

\section{CD162}

C

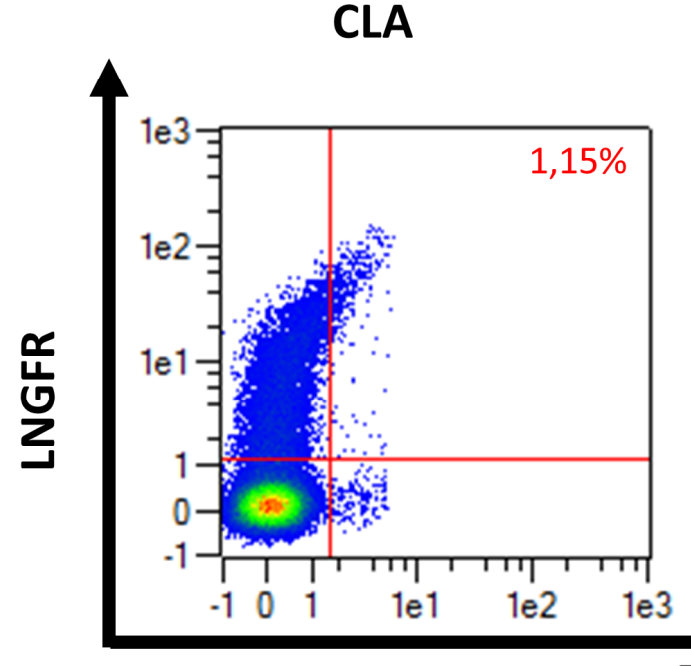

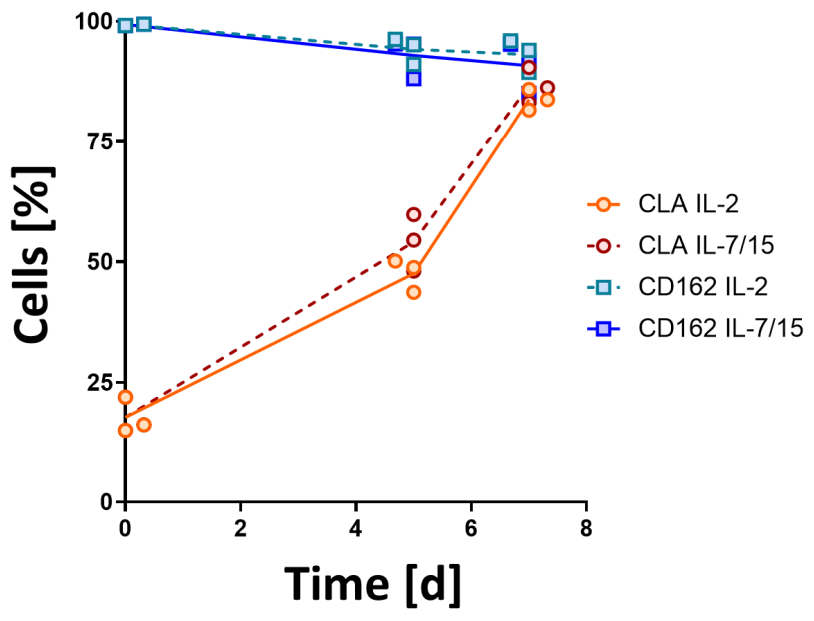

CD318

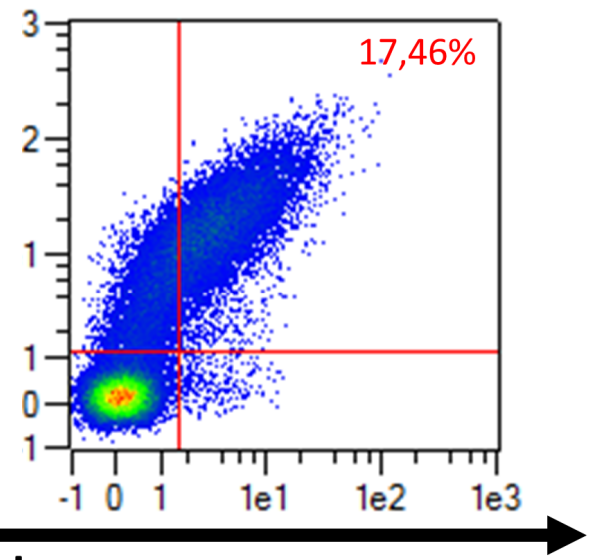

aFab

Figure S8: CLA is a expressed on T cells and upregulated upon activation. (a) Expression of CLA and its protein backbone CD162 on T cells upon activation and expansion for 7 days. (b) Kinetic of CLA and CD162 expression on T cells followed over 7 days. Depicted are the single measurements of three donors. Isolated T cells were activated with $\mathrm{T}$ Cell TransAct ${ }^{\mathrm{TM}}$, human and cultivated in medium either supplemented with $100 \mathrm{IU} / \mathrm{ml} \mathrm{IL}-$ 2 or $12.5 \mathrm{ng} / \mathrm{mL}$ of recombinant human interleukin IL-7 and $12.5 \mathrm{ng} / \mathrm{mL}$ of recombinant human IL-15. (c) Although the CAR cassette is functional based on LNGFR expression, the a anti-CLA CAR is expressed only at low levels in LNGFR ${ }^{\text {high }}$ CAR T cells as compared to a CD318 specific CAR. 
a

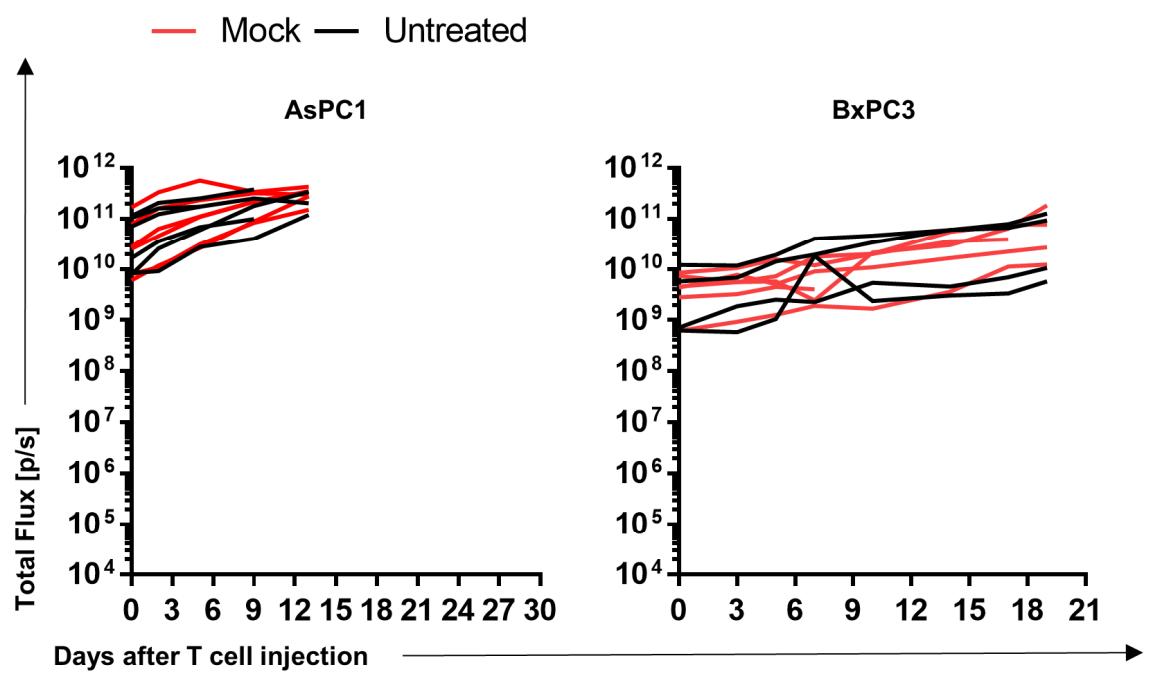

b

BXPC3

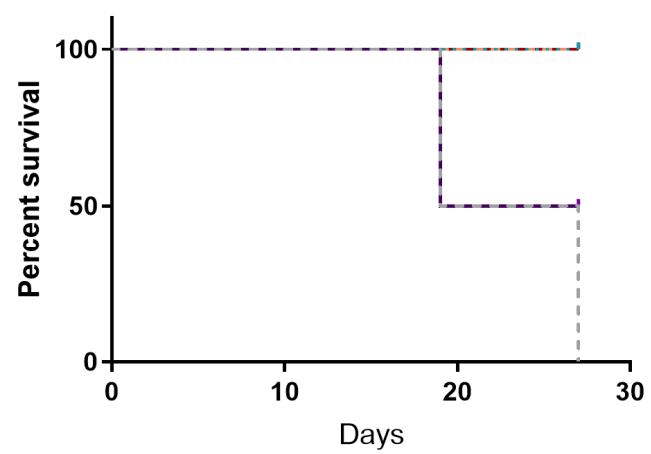

AsPC1

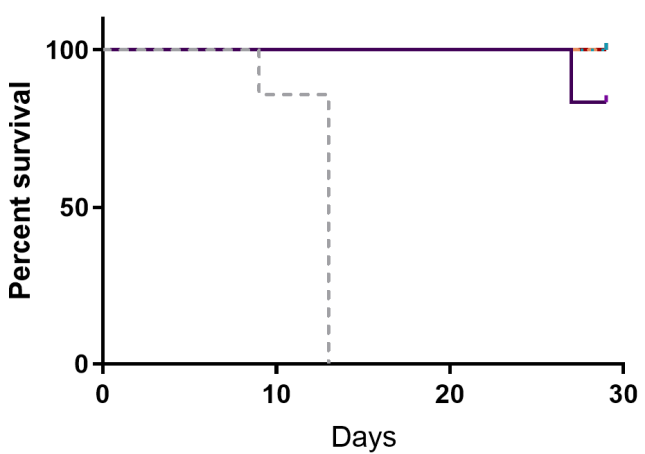

TSPAN8 S - TSPAN8 L

Fig. S9: Mock T cells have no effect on tumor outgrowth in both in vivo models. (a) Tumor burden of mice treated either with Mock T cells or untreated. Left: Mice injected with 1x106 AsPC1 cells (Mock: $n=6$, Untreated: $\mathrm{n}=5$ ). Right: Mice injected with $1 \times 10^{6}$ BxPC3 cells (Mock: $n=6$, Untreated $n=4$ ). (b) Kaplan-Meier plots of mice treated with T cells. Each group included 4-6 mice (animals taken out for interim analysis were not included). 
a
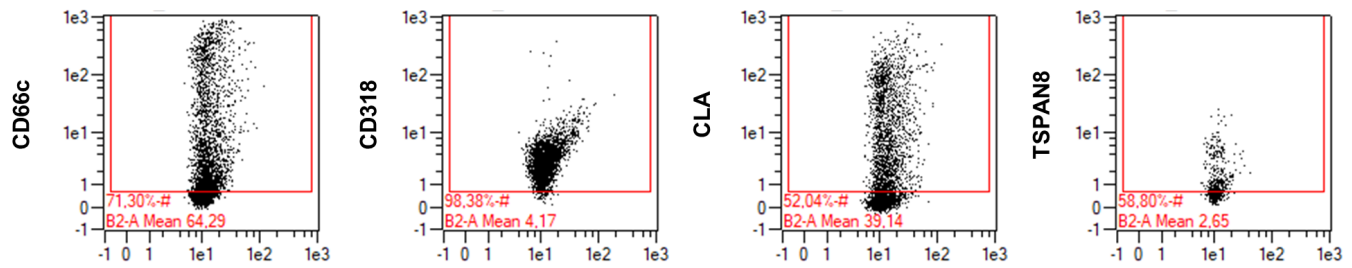

BxPC3
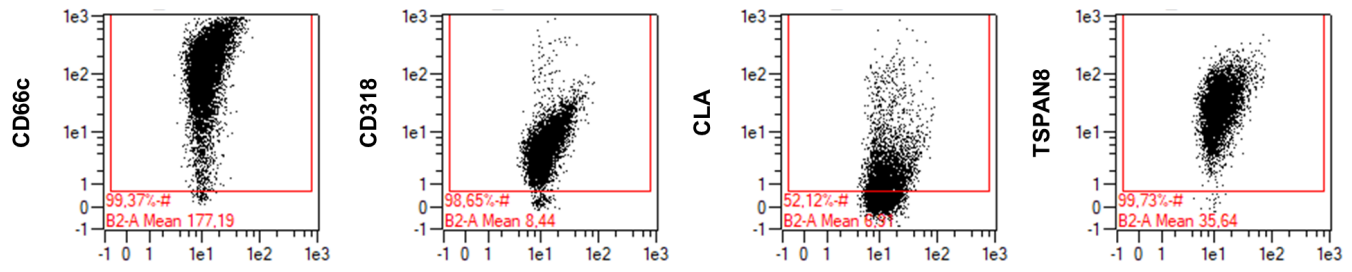

AsPC1

b

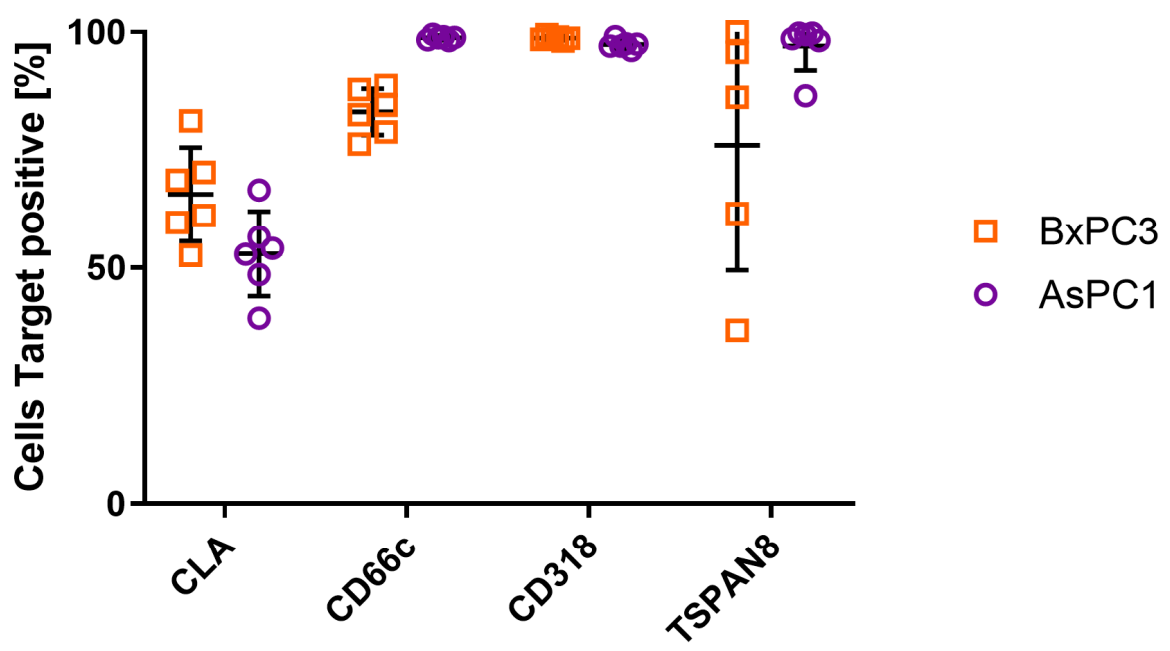

\section{AsPC1}
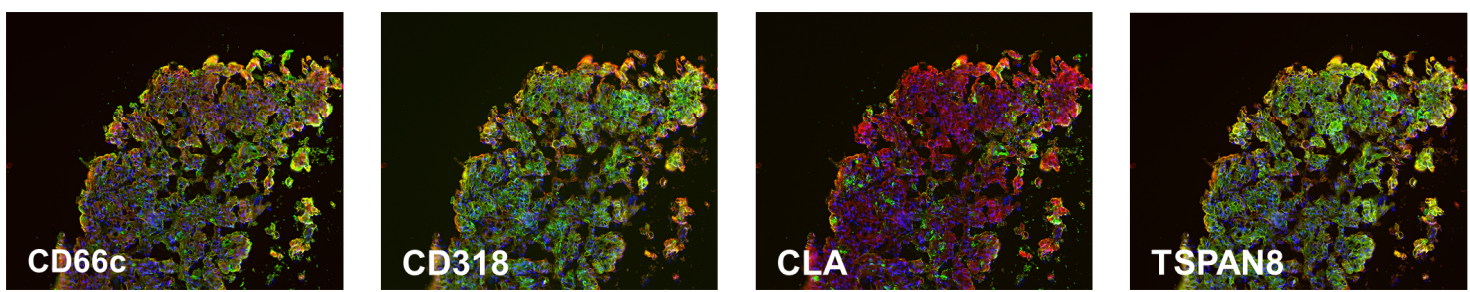

\section{BxPC3}
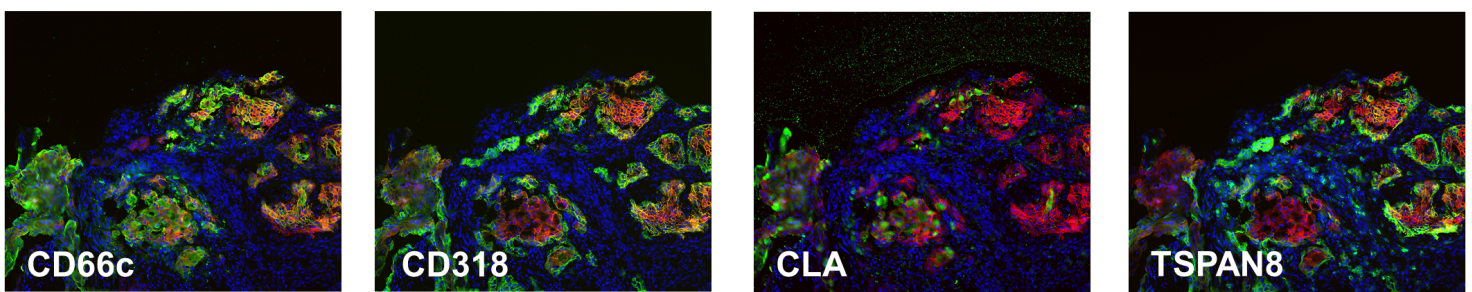

Fig. S10: Analysis of target expression upon xenotransplantation of AsPC1 and BxPC3 cells in vivo. The expression of the target candidates was evaluated upon xenotransplantation of AsPC1 and BxPC3 cells in vivo. Tumors were grown for 30 days, resected and analyzed for target expression by (a, b) flow cytometry, and (c) immunofluorescence $(n=6)$. 


\section{CD318}
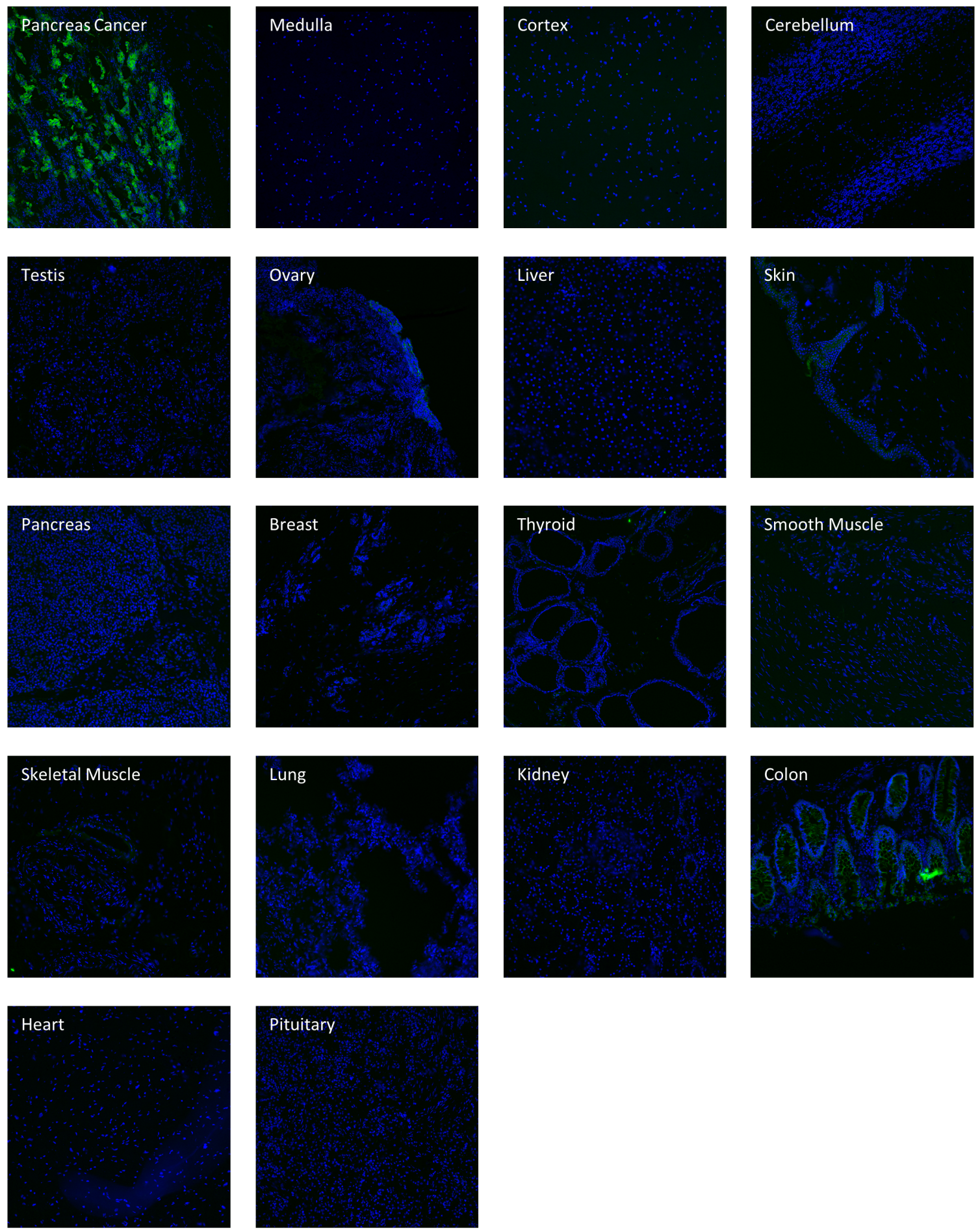

Fig. S11: CD318 expression on healthy tissues assessed by cyclic immunofluorescence imaging. Representative cyclic immune fluorescence images of several healthy tissues stained with a CD318-PE conjugate. 


\section{TSPAN8}
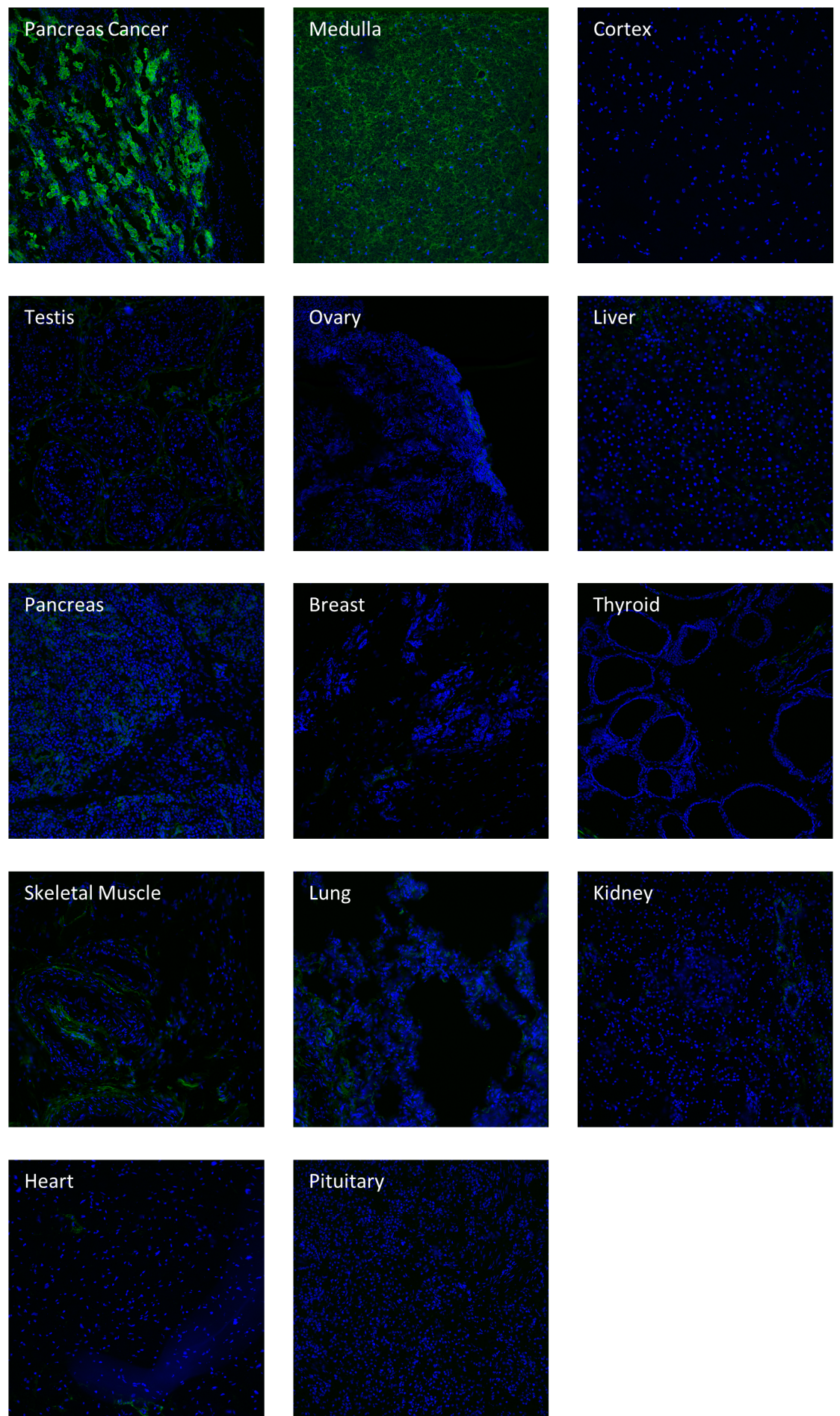

Cerebellum
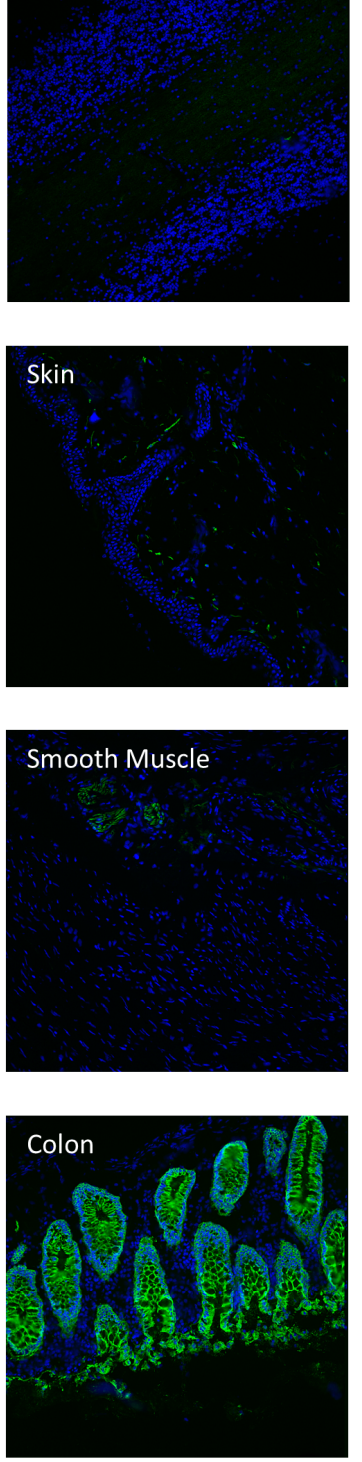

Fig. S12: TSPAN8 expression on healthy tissues assessed by cyclic immunofluorescence imaging. Representative cyclic immune fluorescence images of several healthy tissues stained with a TSPAN8-PE conjugate. 


\section{CD66c}
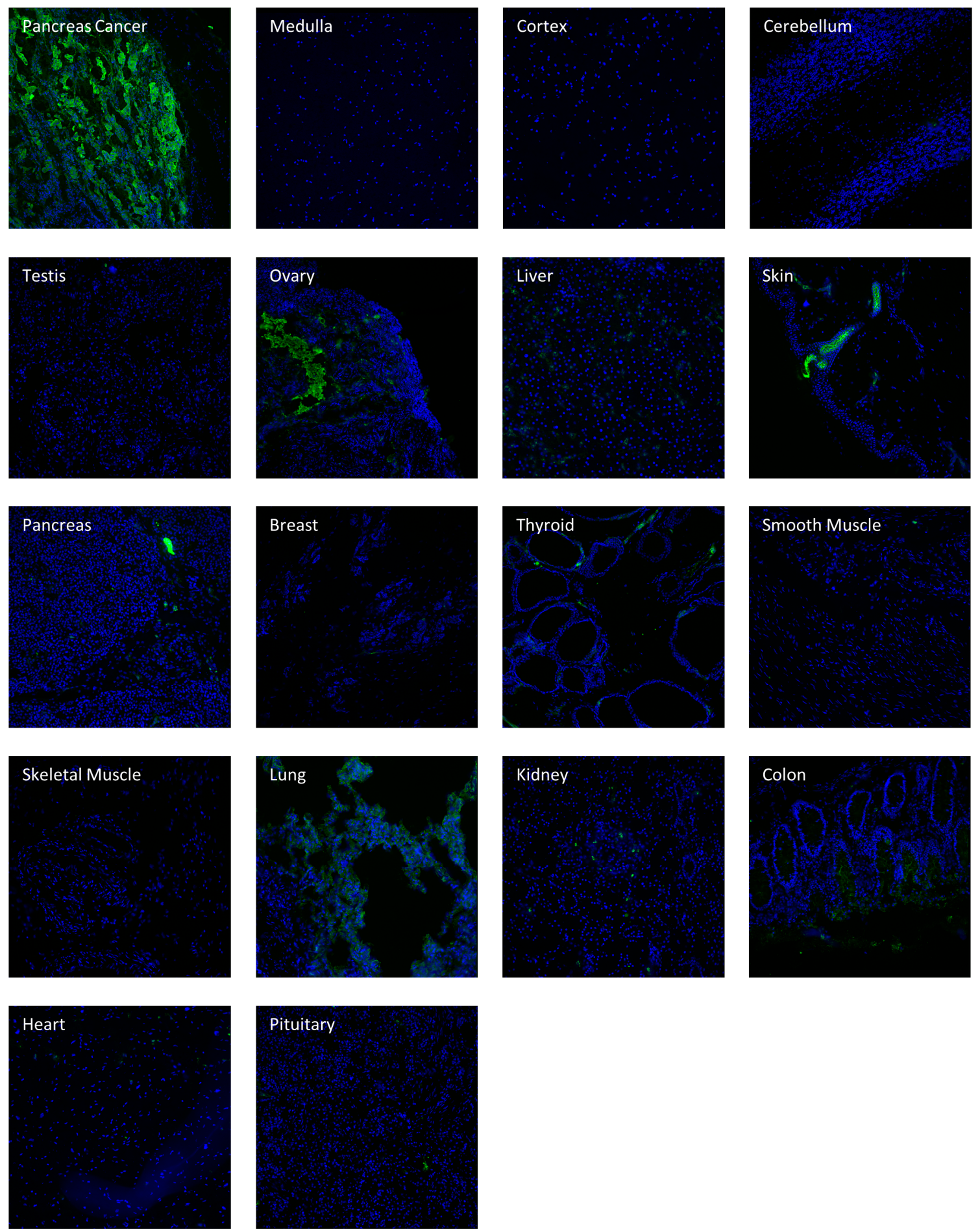

Fig. S13: CD66c expression on healthy tissues assessed by cyclic immunofluorescence imaging. Representative cyclic immune fluorescence images of several healthy tissues stained with a CD66c-PE conjugate. 


\section{References}

1. Siegel RL, Miller KD, Jemal A. Cancer statistics, 2018. CA: A Cancer Journal for Clinicians 2018;68(1):7-30 doi:10.3322/caac.21442.

2. Bray F, Ferlay J, Soerjomataram I, Siegel RL, Torre LA, Jemal A. Global cancer statistics 2018: GLOBOCAN estimates of incidence and mortality worldwide for 36 cancers in 185 countries. CA: A Cancer Journal for Clinicians 2018;68(6):394-424 doi:10.3322/caac.21492.

3. Kleeff J, Korc M, Apte M, La Vecchia C, Johnson CD, Biankin AV, et al. Pancreatic cancer. Nature Reviews Disease Primers 2016;2:16022 doi 10.1038/nrdp.2016.22.

4. Neoptolemos JP, Kleeff J, Michl P, Costello E, Greenhalf W, Palmer DH. Therapeutic developments in pancreatic cancer: current and future perspectives. Nature Reviews Gastroenterology \& Hepatology 2018;15(6):333-48 doi 10.1038/s41575-018-0005-x.

5. Kalos M, Levine BL, Porter DL, Katz S, Grupp SA, Bagg A, et al. T Cells with Chimeric Antigen Receptors Have Potent Antitumor Effects and Can Establish Memory in Patients with Advanced Leukemia. Science Translational Medicine 2011;3(95):95ra73-95ra73 doi 10.1126/scitranslmed.3002842.

6. Davila ML, Riviere I, Wang X, Bartido S, Park J, Curran K, et al. Efficacy and Toxicity Management of 19-28z CAR T Cell Therapy in B Cell Acute Lymphoblastic Leukemia. Science Translational Medicine 2014;6(224):224ra25-ra25 doi 10.1126/scitranslmed.3008226.

7. Kochenderfer JN, Dudley ME, Kassim SH, Somerville RP, Carpenter RO, Stetler-Stevenson M, et al. Chemotherapy-refractory diffuse large B-cell lymphoma and indolent B-cell malignancies can be effectively treated with autologous $\mathrm{T}$ cells expressing an anti-CD19 chimeric antigen receptor. Journal of clinical oncology : official journal of the American Society of Clinical Oncology 2015;33(6):540-9 doi 10.1200/jco.2014.56.2025.

8. Akce M, Zaidi MY, Waller EK, El-Rayes BF, Lesinski GB. The Potential of CAR T Cell Therapy in Pancreatic Cancer. Frontiers in Immunology 2018;9(2166) doi 10.3389/fimmu.2018.02166.

9. Morgan RA, Yang JC, Kitano M, Dudley ME, Laurencot CM, Rosenberg SA. Case report of a serious adverse event following the administration of $\mathrm{T}$ cells transduced with a chimeric antigen receptor recognizing ERBB2. Molecular therapy : the journal of the American Society of Gene Therapy 2010;18(4):843-51 doi 10.1038/mt.2010.24. 
10. Thistlethwaite FC, Gilham DE, Guest RD, Rothwell DG, Pillai M, Burt DJ, et al. The clinical efficacy of first-generation carcinoembryonic antigen (CEACAM5)-specific CAR T cells is limited by poor persistence and transient pre-conditioning-dependent respiratory toxicity. Cancer immunology, immunotherapy : CII 2017;66(11):1425-36 doi 10.1007/s00262017-2034-7.

11. Beatty GL, O'Hara MH, Lacey SF, Torigian DA, Nazimuddin F, Chen F, et al. Activity of Mesothelin-Specific Chimeric Antigen Receptor T Cells Against Pancreatic Carcinoma Metastases in a Phase 1 Trial. Gastroenterology 2018;155(1):29-32 doi 10.1053/j.gastro.2018.03.029.

12. Guedan S, Calderon H, Posey AD, Jr., Maus MV. Engineering and Design of Chimeric Antigen Receptors. Molecular Therapy - Methods \& Clinical Development 2019; 12:145-56 doi 10.1016/j.omtm.2018.12.009.

13. Pompili L, Porru M, Caruso C, Biroccio A, Leonetti C. Patient-derived xenografts: a relevant preclinical model for drug development. Journal of Experimental \& Clinical Cancer Research 2016;35(1):189 doi 10.1186/s13046-016-0462-4.

14. Fuhlbrigge RC, Kieffer JD, Armerding D, Kupper TS. Cutaneous lymphocyte antigen is a specialized form of PSGL-1 expressed on skin-homing T cells. Nature 1997;389:978 doi $10.1038 / 40166$.

15. Ohmori K, Fukui F, Kiso M, Imai T, Yoshie O, Hasegawa H, et al. Identification of cutaneous lymphocyte-associated antigen as sialyl 6-sulfo Lewis X, a selectin ligand expressed on a subset of skin-homing helper memory T cells. Blood 2006;107(8):3197-204 doi 10.1182/blood-2005-05-2185.

16. Yoshino T, Okano M, Chen HL, Tsuchiyama J, Kondo E, Nishiuchi R, et al. Cutaneous lymphocyte antigen is expressed on memory/effector B cells in the peripheral blood and monocytoid B cells in the lymphoid tissues. Cellular immunology 1999;197(1):39-45 doi 10.1006/cimm.1999.1552.

17. Strunk D, Egger C, Leitner G, Hanau D, Stingl G. A skin homing molecule defines the langerhans cell progenitor in human peripheral blood. The Journal of experimental medicine 1997;185(6):1131-6. 
18. Tsuchiyama J, Yoshino T, Toba K, Harada N, Nishiuchi R, Akagi T, et al. Induction and characterization of cutaneous lymphocyte antigen on natural killer cells. British journal of haematology 2002;118(2):654-62.

19. Dzionek A, Fuchs A, Schmidt P, Cremer S, Zysk M, Miltenyi S, et al. BDCA-2, BDCA-3, and BDCA-4: Three Markers for Distinct Subsets of Dendritic Cells in Human Peripheral Blood. The Journal of Immunology 2000;165(11):6037-46 doi 10.4049/jimmunol.165.11.6037.

20. Uhlén M, Fagerberg L, Hallström BM, Lindskog C, Oksvold P, Mardinoglu A, et al. Tissuebased map of the human proteome. Science 2015;347(6220):1260419 doi 10.1126/science. 1260419 .

21. Wilhelm M, Schlegl J, Hahne H, Gholami AM, Lieberenz M, Savitski MM, et al. Massspectrometry-based draft of the human proteome. Nature 2014;509(7502):582-7 doi 10.1038/nature13319.

22. Kim MS, Pinto SM, Getnet D, Nirujogi RS, Manda SS, Chaerkady R, et al. A draft map of the human proteome. Nature 2014;509(7502):575-81 doi 10.1038/nature13302.

23. Perna F, Berman SH, Soni RK, Mansilla-Soto J, Eyquem J, Hamieh M, et al. Integrating Proteomics and Transcriptomics for Systematic Combinatorial Chimeric Antigen Receptor Therapy of AML. Cancer cell 2017;32(4):506-19.e5 doi 10.1016/j.ccell.2017.09.004.

24. Stoiber S, Cadilha BL, Benmebarek M-R, Lesch S, Endres S, Kobold S. Limitations in the Design of Chimeric Antigen Receptors for Cancer Therapy. Cells 2019;8(5):472 doi $10.3390 /$ cells 8050472 .

25. Cummings RD. Structure and function of the selectin ligand PSGL-1. Brazilian journal of medical and biological research $=$ Revista brasileira de pesquisas medicas e biologicas 1999;32(5):519-28.

26. Cooper ML, Choi J, Staser K, Ritchey JK, Devenport JM, Eckardt K, et al. An "off-theshelf" fratricide-resistant CAR- $\mathrm{T}$ for the treatment of $\mathrm{T}$ cell hematologic malignancies. Leukemia 2018;32(9):1970-83 doi 10.1038/s41375-018-0065-5.

27. Gomes-Silva D, Srinivasan M, Sharma S, Lee CM, Wagner DL, Davis TH, et al. CD7edited $\mathrm{T}$ cells expressing a CD7-specific CAR for the therapy of T-cell malignancies. Blood 2017;130(3):285-96 doi 10.1182/blood-2017-01-761320. 
28. Png YT, Vinanica N, Kamiya T, Shimasaki N, Coustan-Smith E, Campana D. Blockade of CD7 expression in $\mathrm{T}$ cells for effective chimeric antigen receptor targeting of $\mathrm{T}$-cell malignancies. Blood advances 2017;1(25):2348-60 doi 10.1182/bloodadvances.2017009928.

29. Scherl-Mostageer M, Sommergruber W, Abseher R, Hauptmann R, Ambros P, Schweifer $\mathrm{N}$. Identification of a novel gene, CDCP1, overexpressed in human colorectal cancer. Oncogene 2001;20(32):4402-8.

30. Kanetaka K, Sakamoto M, Yamamoto Y, Yamasaki S, Lanza F, Kanematsu T, et al. Overexpression of tetraspanin CO-029 in hepatocellular carcinoma. Journal of hepatology 2001;35(5):637-42.

31. Wang H, Rana S, Giese N, Buchler MW, Zoller M. Tspan8, CD44v6 and alpha6beta4 are biomarkers of migrating pancreatic cancer-initiating cells. International journal of cancer 2013;133(2):416-26 doi 10.1002/ijc.28044.

32. Greco C, Bralet MP, Ailane N, Dubart-Kupperschmitt A, Rubinstein E, Le Naour F, et al. E-cadherin/p120-catenin and tetraspanin Co-029 cooperate for cell motility control in human colon carcinoma. Cancer research 2010;70(19):7674-83 doi 10.1158/0008-5472.can-09-4482.

33. Awakura Y, Nakamura E, Takahashi T, Kotani H, Mikami Y, Kadowaki T, et al. Microarray-based identification of CUB-domain containing protein 1 as a potential prognostic marker in conventional renal cell carcinoma. Journal of cancer research and clinical oncology 2008;134(12):1363-9 doi 10.1007/s00432-008-0412-4.

34. Miyazawa Y, Uekita T, Ito Y, Seiki M, Yamaguchi H, Sakai R. CDCP1 regulates the function of MT1-MMP and invadopodia-mediated invasion of cancer cells. Molecular cancer research : MCR 2013;11(6):628-37 doi 10.1158/1541-7786.mcr-12-0544.

35. Ru GQ, Han Y, Wang W, Chen Y, Wang HJ, Xu WJ, et al. CEACAM6 is a prognostic biomarker and potential therapeutic target for gastric carcinoma. Oncotarget 2017;8(48):8367383 doi 10.18632/oncotarget.19415.

36. Duxbury MS, Matros E, Clancy T, Bailey G, Doff M, Zinner MJ, et al. CEACAM6 is a novel biomarker in pancreatic adenocarcinoma and PanIN lesions. Annals of surgery 2005;241(3):491-6. 
37. Guest RD, Hawkins RE, Kirillova N, Cheadle EJ, Arnold J, O'Neill A, et al. The Role of Extracellular Spacer Regions in the Optimal Design of Chimeric Immune Receptors: Evaluation of Four Different scFvs and Antigens. Journal of Immunotherapy 2005;28(3):20311.

38. James SE, Greenberg PD, Jensen MC, Lin Y, Wang J, Till BG, et al. Antigen Sensitivity of CD22-Specific Chimeric TCR Is Modulated by Target Epitope Distance from the Cell Membrane. The Journal of Immunology 2008;180(10):7028-38 doi 10.4049/jimmunol.180.10.7028.

Watanabe N, Bajgain P, Sukumaran S, Ansari S, Heslop HE, Rooney CM, et al. Fine-tuning the CAR spacer improves T-cell potency. Oncoimmunology 2016;5(12):e1253656-e doi 10.1080/2162402X.2016.1253656.

40. Blumenthal RD, Leon E, Hansen HJ, Goldenberg DM. Expression patterns of CEACAM5 and CEACAM6 in primary and metastatic cancers. BMC cancer 2007;7:2 doi 10.1186/14712407-7-2.

41. Caruso HG, Hurton LV, Najjar A, Rushworth D, Ang S, Olivares S, et al. Tuning Sensitivity of CAR to EGFR Density Limits Recognition of Normal Tissue While Maintaining Potent Antitumor Activity. Cancer research 2015;75(17):3505-18 doi 10.1158/0008-5472.can-150139.

42. Muturi-Kioi V, Lewis D, Launay O, Leroux-Roels G, Anemona A, Loulergue P, et al. Neutropenia as an Adverse Event following Vaccination: Results from Randomized Clinical Trials in Healthy Adults and Systematic Review. PloS one 2016;11(8):e0157385 doi 10.1371/journal.pone.0157385.

43. Lanitis E, Poussin M, Klattenhoff AW, Song D, Sandaltzopoulos R, June CH, et al. Chimeric antigen receptor T Cells with dissociated signaling domains exhibit focused antitumor activity with reduced potential for toxicity in vivo. Cancer Immunol Res 2013;1(1):43-53 doi 10.1158/2326-6066.cir-13-0008.

44. Wilkie S, van Schalkwyk MC, Hobbs S, Davies DM, van der Stegen SJ, Pereira AC, et al. Dual targeting of ErbB2 and MUC1 in breast cancer using chimeric antigen receptors engineered to provide complementary signaling. Journal of clinical immunology 2012;32(5):1059-70 doi 10.1007/s10875-012-9689-9. 
45. Fedorov VD, Themeli M, Sadelain M. PD-1- and CTLA-4-based inhibitory chimeric antigen receptors (iCARs) divert off-target immunotherapy responses. Sci Transl Med 2013;5(215):215ra172 doi 10.1126/scitranslmed.3006597.

46. Cartellieri M, Feldmann A, Koristka S, Arndt C, Loff S, Ehninger A, et al. Switching CAR $\mathrm{T}$ cells on and off: a novel modular platform for retargeting of T cells to AML blasts. Blood cancer journal 2016;6(8):e458 doi 10.1038/bcj.2016.61.

47. Ma JS, Kim JY, Kazane SA, Choi SH, Yun HY, Kim MS, et al. Versatile strategy for controlling the specificity and activity of engineered $\mathrm{T}$ cells. Proceedings of the National Academy of Sciences of the United States of America 2016;113(4):E450-8 doi $10.1073 /$ pnas. 1524193113 .

48. Srivastava S, Salter AI, Liggitt D, Yechan-Gunja S, Sarvothama M, Cooper K, et al. LogicGated ROR1 Chimeric Antigen Receptor Expression Rescues T Cell-Mediated Toxicity to Normal Tissues and Enables Selective Tumor Targeting. Cancer cell 2019;35(3):489-503.e8 doi 10.1016/j.ccell.2019.02.003.

49. Bonnet M, Maisonial-Besset A, Zhu Y, Witkowski T, Roche G, Boucheix C, et al. Targeting the Tetraspanins with Monoclonal Antibodies in Oncology: Focus on Tspan8/Co-029. Cancers 2019;11(2):179 doi 10.3390/cancers11020179.

50. Cui Y-H, Kim H, Lee M, Yi JM, Kim R-K, Uddin N, et al. FBXL14 abolishes breast cancer progression by targeting CDCP1 for proteasomal degradation. Oncogene 2018;37(43):5794809 doi 10.1038/s41388-018-0372-3.

51. Kollmorgen G, Niederfellner G, Lifke A, Spohn GJ, Rieder N, Harring SV, et al. Antibody mediated CDCP1 degradation as mode of action for cancer targeted therapy. Molecular oncology 2013;7(6):1142-51 doi 10.1016/j.molonc.2013.08.009.

52. Parkhurst MR, Yang JC, Langan RC, Dudley ME, Nathan D-AN, Feldman SA, et al. T cells targeting carcinoembryonic antigen can mediate regression of metastatic colorectal cancer but induce severe transient colitis. Molecular therapy : the journal of the American Society of Gene Therapy 2011;19(3):620-6 doi 10.1038/mt.2010.272.

53. Katz SC, Burga RA, McCormack E, Wang LJ, Mooring W, Point GR, et al. Phase I Hepatic Immunotherapy for Metastases Study of Intra-Arterial Chimeric Antigen Receptor-Modified T-cell Therapy for CEA+ Liver Metastases. Clin Cancer Res 2015;21(14):3149-59 doi 10.1158/1078-0432.CCR-14-1421. 
54. Hruz T, Laule O, Szabo G, Wessendorp F, Bleuler S, Oertle L, et al. Genevestigator V3: A Reference Expression Database for the Meta-Analysis of Transcriptomes. Advances in Bioinformatics 2008;2008:5 doi 10.1155/2008/420747.

55. Kamentsky L, Jones TR, Fraser A, Bray M-A, Logan DJ, Madden KL, et al. Improved structure, function and compatibility for CellProfiler: modular high-throughput image analysis software. Bioinformatics 2011;27(8):1179-80 doi 10.1093/bioinformatics/btr095. 


\title{
4. Chapter 2 - A Novel Siglec-4 Derived Spacer Improves the Functionality of CAR T Cells Against Membrane-Proximal Epitopes
}

Published in: Schäfer, D., Henze, J., Pfeifer, R., Schleicher, A., Brauner, J., Mockel-Tenbrinck, N., et al. (2020). A Novel Siglec-4 Derived Spacer Improves the Functionality of CAR T Cells Against Membrane-Proximal Epitopes. Frontiers in Immunology 11(1704). doi: 10.3389/fimmu.2020.01704.

Authors: Daniel Schäfer ${ }^{1,2 \dagger}$, Janina Henze ${ }^{1,2 \dagger}$, Rita Pfeifer $^{2 \dagger}$, Anna Schleicher ${ }^{3}$, Janina Brauner $^{2}$, Nadine Mockel-Tenbrinck ${ }^{2}$, Carola Barth ${ }^{2}$, Daniela Gudert ${ }^{2}$, Wa'el Al Rawashdeh ${ }^{2 * \ddagger}$, Ian C. D. Johnston ${ }^{2 * \ddagger}$ and Olaf Hardt ${ }^{2 * \ddagger}$

\section{Affilations:}

${ }^{1}$ Translational Molecular Imaging, Institute for Diagnostic and Interventional Radiology \& Clinic for Hematology and Medical Oncology, University Medical Center Göttingen, Göttingen, Germany

${ }^{2}$ R\&D Reagents, Miltenyi Biotec B.V. \& Co. KG, Bergisch Gladbach, Germany

${ }^{3}$ Faculty of Chemistry and Biosciences, Karlsruher Institute of Technology, Karlsruhe, Germany

${ }^{\dagger}$ These authors share first authorship

‡These authors share last authorship

*Corresponding authors

\begin{abstract}
A domain that is often neglected in the assessment of chimeric antigen receptor (CAR) functionality is the extracellular spacer module. However, several studies have elucidated that membrane proximal epitopes are best targeted through CARs comprising long spacers, while short spacer CARs exhibit highest activity on distal epitopes. This finding can be explained by the requirement to have an optimal distance between the effector $\mathrm{T}$ cell and target cell. Commonly used long spacer domains are the $\mathrm{CH} 2-\mathrm{CH} 3$ domains of $\mathrm{IgG}$ molecules. However, CARs containing these spacers generally show inferior in vivo efficacy in mouse models compared to their observed in vitro activity, which is linked to unspecific Fc $\gamma$-Receptor binding and can be abolished by mutating the respective regions. Here, we first assessed a CAR therapy targeting membrane proximal CD20 using such a modified long IgG1 spacer. However, despite these mutations, this construct failed to unfold its observed in vitro cytotoxic potential in an in vivo model, while a shorter but less structured CD8 $\alpha$ spacer CAR showed complete tumor
\end{abstract}


clearance. Given the shortage of well-described long spacer domains with a favorable functionality profile, we designed a novel class of CAR spacers with similar attributes to IgG spacers but without unspecific off-target binding, derived from the Sialic acid-binding immunoglobulin-type lectins (Siglecs). Of five constructs tested, a Siglec-4 derived spacer showed highest cytotoxic potential and similar performance to a CD8 $\alpha$ spacer in a CD20 specific CAR setting. In a pancreatic ductal adenocarcinoma model, a Siglec-4 spacer CAR targeting a membrane proximal (TSPAN8) epitope was efficiently engaged in vitro, while a membrane distal (CD66c) epitope did not activate the T cell. Transfer of the TSPAN8 specific Siglec-4 spacer CAR to an in vivo setting maintained the excellent tumor killing characteristics being indistinguishable from a TSPAN8 CD8 $\alpha$ spacer CAR while outperforming an IgG4 long spacer CAR and, at the same time, showing an advantageous central memory CAR T cell phenotype with lower release of inflammatory cytokines. In summary, we developed a novel spacer that combines cytotoxic potential with an advantageous $\mathrm{T}$ cell and cytokine release phenotype, which make this an interesting candidate for future clinical applications.

\section{Introduction}

The unprecedented therapeutic efficacy of CAR T cells in previously refractory blood cancers is considered to be one of the major breakthroughs in cancer immunotherapy, culminating in the recent market approvals by the Food and Drug Administration (FDA) and the European Medicines Agency (EMA) for two CAR T cell products (1-7). While CAR therapies have now achieved public recognition, their development and the quest for optimal CAR design has been a multistep process stretching over several decades. Ever since their initial description in 1989 by Eshhar et al. (8), the receptors have evolved from a two-chimeric-TCR chain architecture to a one-protein design. This design commonly incorporates a single-chain variable fragment ( $\mathrm{scFv}$ ) of a given antibody as the antigen binding moiety, an extracellular spacer and a transmembrane region as structural features, as well as signal transduction units for $\mathrm{T}$ cell activation. Originally, the spacer domain was introduced into the CAR framework as an inert building block to allow the antigen binding moiety to extend beyond the T cell's glycocalyx and improve antigen accessibility (9). Following this assumption, a plethora of spacer regions were designed simultaneously ranging from the immunoglobulin (Ig) domains of the crystallizable fragments $(\mathrm{Fc})$ of antibodies to extracellular domains of $\mathrm{CD} 8 \alpha, \mathrm{CD} 28$, the TCR $\beta$ chain or NKG2D (10-16) and were applied without comparative analyses. However, already very early on, Patel and colleagues provided the scientific proof that the spacer region can be of paramount importance for the receptor function and affects its expression, surface stability through the turnover rate, and signal transduction (17). More recent accumulating research has further been 
showing that in addition to the nature of the spacer, effective antigen recognition depends on the functional interplay between the spatial localization of the target epitope and the CAR spacer length (18-20). For instance, membrane-distal epitopes were shown to most efficiently trigger CARs with short spacers, while membrane-proximal epitopes required receptors with extended spacer domains to elicit accurate effector function, in this way emphasizing the biological requirement of optimal T cell-target cell distance (18-22). Thus, the design of CARs against novel antigens needs to consider both the epitope position within the target antigen as well as the nature and length of the spacer region and customize these variables accordingly.

The use of Ig-derived spacers is particularly attractive as it provides the opportunity to modulate the spacer length into long ( $\mathrm{CH} 2-\mathrm{CH} 3$ domain), medium $(\mathrm{CH} 3)$ and short (hinge only) structures, while retaining the nature of the parent protein. However, Ig-derived spacers have faced various complications during their development. In particular, off-target activation, CAR $\mathrm{T}$ cell sequestration in the lung, tonic signaling and activation-induced cell death (AICD) have been described leading to only a limited T cell persistence (23-26). Although these effects could be abrogated by mutating the amino acid sequence essential for FcR binding $(23,25,27)$, it needs to be taken into consideration that these experiments were conducted in immunosuppressed NSG mice and whether FcR binding can be entirely eliminated in humans remains unclear. Of note, several clinical studies that used IgG-derived spacers described only limited anti-tumor efficacy and low CAR T cell persistence (28-31) while others are showing some promising clinical responses (32-34). Interestingly, the first commercially available CAR T cell-based therapies use CD28 (Yescarta) and CD8 (Kymriah) derived spacer domains.

Taking into account the shortage of well-described long spacer domains with a favorable functionality profile, we endeavored to develop a novel long spacer for membrane-proximal epitopes, which naturally lacks an FcR binding domain. Based on the postulated spatial requirements between CARs and their target antigens, we anticipated finding a CAR spacer construct whose functionality against membrane-proximal epitopes extends beyond that of a CD8 $\alpha$ spacer CAR. Hence, we generated novel CAR spacers and analyzed their efficacy sideby-side to the cognate CD8 $\alpha$ spacer counterpart - a comparison that has not been extensively undertaken thus far. The design of the novel spacers was based on the sialic acid binding Iglike lectin (Siglec) receptor family, whose members are broadly expressed on various immune cells $(35,36)$. Structurally, each receptor member is composed of an $\mathrm{N}$-terminal Ig-like V-set domain which is involved in sialic acid binding and a defined number of Ig-like C2-set domains that serve as a structural spacer and extend the binding moiety away from the plasma membrane. 
The selection of the Siglec family was inspired by the hypothesis that the incorporation of naturally occurring spacer domains into the CAR architecture will preserve the biological requirements of a spacer region and minimize unspecific interactions with other cells.

In this study, we confirm this strategy of using naturally occurring spacer domains by first demonstrating, that in a CD20 lymphoma model a long IgG1 spacer CAR is as functional as the CD8 $\alpha$ spacer in vitro, but fails to translate its effectiveness in vivo, despite containing the earlier reported mutations to abrogate FcR binding (23). Subsequently, we evaluate novel spacers derived from the Siglec family of proteins and identify a long alternative spacer derived from Siglec-4 that performs with equal efficiency to the CD8 $\alpha$ spacer in vitro. Finally, we demonstrate in a solid tumor model that the novel Siglec-4 spacer CAR does not exceed, but rather matches the CD8 $\alpha$ spacer CAR cytotoxic activity in vivo on membrane-proximal targets, while maintaining a favorable cell phenotype profile and cytokine release pattern.

\section{Materials and Methods}

\section{CAR Gene Construction}

Commercial gene synthesis in combination with an optimization algorithm for codon usage in humans (ATUM) was used to construct the CAR genes of interest. The CD20-specific scFv was derived from the murine monoclonal antibody Leu16 as originally described by Jensen and colleagues (37), while the CD66c- and TSPAN8-targeting scFv sequences were derived from the antibody clones REA414 (CD66c) and REA443 (TSPAN8) (Miltenyi Biotec). All antigen binding domains contained a $\left(\mathrm{G}_{4} \mathrm{~S}\right)_{3}$-linker between the $\mathrm{V}_{\mathrm{L}}$ and the $\mathrm{V}_{\mathrm{H}}$ regions. To facilitate receptor trafficking to the plasma membrane, a human CD8 $\alpha$ leader signaling peptide was added $\mathrm{N}$-terminally to the respective $\mathrm{scFv}$ sequence. The spacer region downstream of the $\mathrm{scFv}$ encompassed either the domain for IgG1 hinge-CH2CH3 (234 amino acids), IgG4 hinge$\mathrm{CH} 2 \mathrm{CH} 3$ (228 amino acids), or CD8 $\alpha$ hinge (45 amino acids). To abrogate potential interactions of the Fc spacer CARs with FcR-expressing cells, the PELLGG and ISR motives in the IgG1 $\mathrm{CH} 2$ domain were replaced by the corresponding IgG2 amino acids (23). In the case of the IgG4 $\mathrm{CH} 2$ domain, the APEFLG sequence was replaced by APPVA from IgG2 and an N279Q mutation was introduced to remove glycosylation at this site (25). Spacers derived from the Siglec family were designed based on the protein sequences extracted from UniProt and the plasma membrane-proximal domains were incorporated into the CAR architecture. Thus, the Siglec-3 spacer comprised the amino acids 145-259 of the parent protein with a C169S mutation to abrogate unspecific disulfide-bond formation. The Siglec-4 spacer contained the amino acids 238-519, the Siglec-7.1 spacer the amino acids 150-353, the Siglec- 
7.2 spacer the amino acids $234-353$, and the Siglec-8 spacer the amino acids $241-363$ of the respective parent protein. All spacers were linked to the transmembrane domain of human $\mathrm{CD} 8 \alpha$, the intracellular domain of $4-1 \mathrm{BB}$, and the $\mathrm{CD} 3 \zeta$ signaling domain as derived from UniProt. The $C A R$ genes were fused to a Furin-P2A sequence to include co-expression of the truncated low affinity nerve growth factor receptor ( $\triangle$ LNGFR). Transgene expression was promoted by the PGK promoter located upstream of the $C A R$ gene.

\section{Lentiviral Vector Production}

Second generation self-inactivating VSV-G-pseudotyped lentiviral vectors were produced by transient transfection of adherent HEK293T cells. One day before transfection, $1.6 \times 10^{7}$ HEK293T cells were seeded per T175 flask to reach a confluency of 70-90\% on the following day. Each T175 flask was then transfected with a total of $35 \mu \mathrm{g}$ plasmid DNA composed of pMDG2 (encoding VSV-G), pCMVdR8.74 (encoding gag/pol), and the respective transgeneencoding transfer vector using MACSfectin reagent (Miltenyi Biotec). All transfection reactions were performed with a DNA: MACSfectin ratio of 1:2. Following overnight incubation, sodium butyrate was supplied at a final concentration of $10 \mathrm{mM}$ and at $48 \mathrm{~h}$ after transfection the medium was collected, cleared by centrifugation at $300 \times \mathrm{g}$ and $4^{\circ} \mathrm{C}$ for 5 min and filtered through $0.45 \mu \mathrm{m}$-pore-size PVDF filters. Concentration of the viral stock was performed by centrifugation at $4^{\circ} \mathrm{C}$ and $4,000 \times \mathrm{g}$ for $24 \mathrm{~h}$. Pellets containing lentiviral vector were air-dried and resuspended at a 100 -fold concentration with $4^{\circ} \mathrm{C}$ cold PBS. Lentiviral vector aliquots were stored at $-80^{\circ} \mathrm{C}$.

\section{Generation of CAR T Cells}

\section{Automated CAR T Cell Generation}

The CliniMACS Prodigy® TCT (T cell transduction) application was used for the automated manufacturing of large amounts of gene-modified T cells. Technical features and experimental procedures have previously been described in detail $(38,39)$. In brief, T cells were obtained from non-mobilized leukapheresis from healthy anonymous donors (University Hospital Cologne or the German Red Cross Ulm) and were typically processed 24-48 h after collection. Transduced and enriched CAR T cells were finally formulated and harvested in Composol ${ }^{\circledR}$ solution (Fresenius Kabi), supplemented with $2.5 \%$ human serum albumin (Grifols). For quality assurance, the transduction efficiency and $\mathrm{T}$ cell phenotype was determined using a MACSQuant Analyzer 10 (Miltenyi Biotec) after the TCT process. Transduction efficiency 
were determined by flow cytometry on days 5 and 12 of the TCT process using a flow cytometer.

\section{Manual CAR T Cell Generation}

Buffy coats from healthy anonymous donors were obtained from the German Red Cross Dortmund. Peripheral blood mononuclear cells (PBMCs) were then isolated from buffy coats by density gradient centrifugation. T cells were purified from PBMCs applying the Pan T Cell Isolation Kit, human (Miltenyi Biotec) and activated in TexMACS ${ }^{\mathrm{TM}}$ Medium (Miltenyi Biotec) supplemented with T Cell TransAct ${ }^{\mathrm{TM}}$, human (Miltenyi Biotec) and $100 \mathrm{IU} / \mathrm{ml}$ of recombinant Human IL-2 IS, research grade (Miltenyi Biotec). T cells were transduced $24 \mathrm{~h}$ after activation using VSV-G pseudotyped lentiviral particles. 3 days post activation, T Cell TransAct ${ }^{\mathrm{TM}}$, human and excess viral vector were removed and $\mathrm{T}$ cells were cultured in TexMACS ${ }^{\mathrm{TM}}$ Medium only supplemented with IL-2. T cells were expanded for 12 days and used directly for in vitro assays or frozen in TexMACS ${ }^{\mathrm{TM}}$ Medium containing $10 \%$ DMSO for later in vivo use. Frozen T cells that were used for in vivo testing were thawed $24 \mathrm{~h}$ before injection and cultivated at $37^{\circ} \mathrm{C}$ in TexMACS ${ }^{\mathrm{TM}}$ Medium without further supplements.

\section{Target Cell Lines}

HEK293T, JeKo-1, Raji and AsPC1 cells were obtained from ATCC and cultured as recommended. Raji cells were transduced with a ffLuc cassette for in vivo detection and AsPC1 cells were transduced with a eGFP/ffLuc cassette for in vitro and in vivo detection. To validate authenticity of the cell lines used, we used the Human STR Profiling Cell Authentication Service (ATCC).

\section{Flow Cytometry}

Antibodies specific for anti-human CD62L, CD45RO, CD95, CD271 (LNGFR), CD107a, TNF- $\alpha$, CD223 (LAG3), CD279 (PD1), CD366 (TIM3), CD137 (4-1BB), CD4, CD8, CD3 were monoclonal recombinant antibodies (Miltenyi Biotec). For anti-CD20 CAR detection the CD20 CAR Detection Reagent (Miltenyi Biotec) was used. Staining of Miltenyi Biotec antibodies was performed according to the supplier's instructions. For direct CAR detection of CD66c and TSPAN8 specific CARs a sequential staining was used. First, samples were incubated with polyclonal Fab specific anti-mouse IgG antibodies produced in goat (Merck) at concentrations of $10 \mu \mathrm{g} / \mathrm{ml}$ for $30 \mathrm{~min}$ at $4^{\circ} \mathrm{C}$. Samples were washed and then incubated with polyclonal anti-goat IgG antibodies produced in chicken (Thermo Fisher) at concentrations of $10 \mu \mathrm{g} / \mathrm{ml}$ for $30 \mathrm{~min}$ at $4^{\circ} \mathrm{C}$. Stained samples were measured on a MACSQuant ${ }^{\circledR}$ Analyzer 8 or 
MACSQuant Analyzer 10 (Miltenyi Biotec) and analyzed using the MACSQuantify ${ }^{\mathrm{TM}}$ Software.

\section{In vitro Functional Assays}

\section{With JeKo-1 Target Cells}

$1 \times 10^{5}$ JeKo- 1 and $1 \times 10^{5}$ CAR T cells were co-cultured in TexMACS ${ }^{\mathrm{TM}}$ Medium (Miltenyi Biotec) for $24 \mathrm{~h}$ in 96-well round bottom plates. Supernatants were collected at the endpoint and used to detect the cytokines released by anti-CD20 CAR T cells using the MACSPlex Cytokine 12 Kit (Miltenyi Biotec) with the four selected human cytokines IFN- $\gamma$, IL-2, TNF- $\alpha$ and GM-CSF, according to the manufacturer's instructions. The cytolytic activity of the engineered T cells was evaluated by using $1 \times 10^{4} \mathrm{CD} 20^{+}$JeKo- 1 cells labeled with $1 \mu \mathrm{M}$ CellTrace $^{\mathrm{TM}}$ Violet (Life Technologies), as target cells. Effector and target cells were cocultured for $24 \mathrm{~h}$ at the indicated ratios (E:T) in 96-well round bottom plates. Detection of the specific lysis was performed by quantitation of Violet dye labeled target cells using a MACSQuant Analyzer 8 (Miltenyi Biotec). Mock-transduced T cells were used as control at the same effector-to-target ratios.

\section{With Raji Cells}

$2 \times 10^{5}$ CAR T cells were incubated with $2 \times 10^{5} \mathrm{CD}^{2} 0^{+}$Raji cells in $200 \mu \mathrm{l}$ TexMACS ${ }^{\mathrm{TM}}$ Medium at $37^{\circ} \mathrm{C}$. In addition, the medium was supplemented with $20 \mu \mathrm{l}$ of a CD107a specific antibody. After $1 \mathrm{~h}$ of incubation the protein transport inhibitors Monensin and Brefeldin A (BD Biosiences) were added as recommended for $4 \mathrm{~h}$. After this incubation period, cells were washed and first surface stained with LNGFR specific antibodies to label transduced $\mathrm{T}$ cells and subsequently intracellularly stained for TNF- $\alpha$ using the Inside Stain Kit and a TNF- $\alpha$ specific antibody (all Miltenyi Biotec). Cells were then measured by flow cytometry. For TIM3, LAG3 and PD1 detection $1 \times 10^{5}$ CAR T cells were inoculated with $2 \times 10^{5} \mathrm{CD} 20^{+}$Raji cells in $200 \mu \mathrm{l}$ TexMACS ${ }^{\text {тм }}$ Medium at $37^{\circ} \mathrm{C}$ for $24 \mathrm{~h}$. Subsequently $\mathrm{T}$ cells were stained and analyzed by flow cytometry.

For functionality assays in the presence of NSG macrophages, $2 \times 10^{5}$ CAR T cells were incubated in a 1:1:1 ratio with Raji target cells and macrophages derived from a peritoneal lavage. The assay was performed in the presence or absence of murine FcR-blocking reagent. After $24 \mathrm{~h}$ of incubation, detection of the specific lysis was performed by quantitation of Violet dye labeled target cells via flow cytometry using a MACSQuant Analyzer 8 (Miltenyi Biotec). 


\section{With AsPC1 Cells}

$\mathrm{GFP}^{+} / \mathrm{Luc}^{+} \mathrm{AsPC} 1$ target cells were inoculated in 96-well plates at $2.5 \times 10^{4}$ cells per well in TexMACS ${ }^{\mathrm{TM}}$ Medium. CAR T cells or untransduced Mock T cells were added with at an E:T ratio of 2:1. The amount of $\mathrm{T}$ cells in the Mock control was adjusted to the number of total $\mathrm{T}$ cells in the CAR group with the highest total cell count. Cytotoxicity was measurement as the decrease of green surface area as assessed by the IncuCyte ${ }^{\circledR}$ S3 Live-Cell Analysis System (Sartorius). Measured values were normalized to the start of the experiment. After $24 \mathrm{~h}$ a supernatant sample was taken for cytokine measurements using the MACSPlex Cytokine 12 Kit. At the end of the experiment expression of LAG3, PD1, and 4-1BB were measured using a MACSQuant Analyzer 8 (Miltenyi Biotec). Specific endpoint killing was calculated from the green surface area values with the following formula:

$$
\text { specific killing }[\%]=100-(100 * \text { green area Mockgreen area CAR }) \text {. }
$$

\section{In vivo Assays}

Experiments involving animal handling were approved by the Governmental Review Committee on Animal Care in NRW, Germany and performed according to guidelines and regulations (Landesamt für Natur, Umwelt and Verbraucherschutz NRW, Approval number 8402.04.2015.A168 and Approval number 84-02.04.2017.A021).

Raji lymphoma were established by tail vein injection of $5 \times 10^{5} \mathrm{Raji}_{\mathrm{Luc}}^{+}$cells. After 7 days, $1 \times 10^{6}$ CAR T cells or Mock GFP-transduced T cells, adjusted to the total amount of $\mathrm{T}$ cells according to transduction efficiency of the CARs, were infused intravenously.

For AsPC1 GFP ${ }^{+} / \mathrm{Luc}^{+}$cell line derived tumors $1 \times 10^{6}$ cells were injected subcutaneously in the right flank of NOD SCID gamma (NSG; NOD.Cg-PrkdcscidIl2rgtm1Wjl/SzJ) mice (Jackson Laboratory, provided by Charles River). When tumors reached a size of $25 \mathrm{~mm}^{2}, 5 \times$ $10^{6} \mathrm{CAR}$ T cells were injected into the tail vein. The amount of injected untransduced Mock T cells was adjusted to the number of total $\mathrm{T}$ cells in the CAR group with the highest total cell count.

Therapeutic response was measured longitudinally using the IVIS Lumina in vivo imaging system (PerkinElmer) after intraperitoneal injection of $100 \mu \mathrm{L}(30 \mathrm{mg} / \mathrm{mL}) \mathrm{D}$-Luciferin (for Raji studies: XenoLight Rediject D-Luciferin Ultra (PerkinElmer). For AsPc1 studies: Potassium Salt, LUCK-2G, GoldBio) and additionally by manual caliper measurement for pancreatic tumors. All measures to secure the well-being of mice were executed following the 
relevant animal use guidelines and ethical regulations. Upon reaching the endpoint (weight loss of $>19 \%$, paralysis, stress score of $>20$ or end-point of the experiment, Day 20 for the lymphoma model and Day 29 for the pancreatic model), animals were euthanized according to guidelines and post-mortem analysis was performed in order to determine tumor burden, persistence and killing of the different CAR T cell constructs. In particular blood, bone marrow and spleen were subjected to flow cytometric analysis. Therefore, spleen was dissociated using the gentleMACS ${ }^{\mathrm{TM}}$ Octo Dissociator with Heaters according to the manufacturers protocol (Miltenyi Biotec) and bone marrow was extracted from the femurs and tibias of mice by cutting off the epiphyses of the bones and rinsing the inner fragments. The cell suspensions were filtered through a $70 \mu \mathrm{m}$ pore size MACS SmartStrainer (Miltenyi Biotec) and following red blood cell lysis on blood, bone marrow and spleen single cell suspensions using Red Blood Cell Lysis Solution (Miltenyi Biotec), samples were stained and analysis was conducted on a MACSQuant Analyzer 8.

\section{Statistics}

Unless otherwise specified, all graphical error bars represent standard error of the mean. Statistical comparisons between more than two groups were conducted by One-way ANOVA with $p<0.05$ using GraphPad Prism 7. To facilitate the statistical overview of the in vivo experiments, the significance analyses were organized in a pairwise significance matrix (PSM) where each box represents a comparison between two groups, as shown by Al Rawashdeh et al. (40). The order, in which the groups were compared, is illustrated in Figures S1, S4. Significant differences between two comparing groups are defined by a green box, while insignificant differences by a red box.

\section{Results}

\section{CD20 Specific CD8a and IgG1 CH2-CH3 Spacer CARs Exhibit Comparable in vitro Activity}

During pre-clinical development of a CD20 directed CAR candidate (39) we also evaluated a number of different CAR configurations (Figure 1A). We used an $\mathrm{scFv}$ derived from the Leu16 monoclonal antibody $(30,41)$, binding to the large extracellular loop of CD20 (42). This loop is only 47 amino acids long, which is why we hypothesized it would be more effectively targeted with a flexible CD8 $\alpha$ or long IgG spacer. We generated two second generation CAR constructs, that comprised a CD8 $\alpha$ transmembrane domain, a 4-1BB co-stimulatory domain and

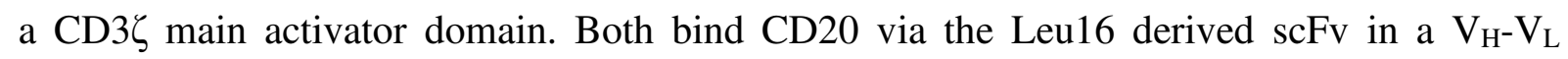
orientation and only differed in the spacer domain. The CD20_hl_IgG1 CAR comprises an IgG1 CH2-CH3 spacer while the CD20_hl_CD8 CAR possesses a CD8 $\alpha$ spacer. The PELLGG 
and ISR motif of the IgG1 CH2-CH3 spacer were replaced by the corresponding IgG2 amino acids to reduce $\mathrm{Fc} \gamma$-Receptor binding, as described previously (23). To assess whether the order of binding domains in the scFv also can play a role in CAR function, we constructed a CD8 $\alpha$ spacer CAR with swapped scFv orientation (CD20_lh_CD8). We generated CD20 specific CAR T cells by genetically modifying CD3/CD28 polyclonally activated $\mathrm{T}$ cells with lentiviral vectors in a fully automated manner in a closed system using the CliniMACS Prodigy ${ }^{\circledR}$ as described previously (39). At the end of the manufacturing on day 12, similar T cell phenotypes were obtained for the samples modified with the different CAR constructs and the untransduced Mock control (Figure 1B). More than $80 \%$ of T cells had a memory phenotype (central memory $\mathrm{T}$ cell $\left(\mathrm{T}_{\mathrm{CM}}\right)$ and stem cell memory $\mathrm{T}$ cell $\left(\mathrm{T}_{\mathrm{SCM}}\right)$ as defined by their phenotypes being $\mathrm{CD} 2 \mathrm{~L}^{+} / \mathrm{CD} 45 \mathrm{RO}^{+} / \mathrm{CD}^{+} 5^{+}$and $\mathrm{CD} 2 \mathrm{~L}^{+} / \mathrm{CD} 45 \mathrm{RO}^{-} / \mathrm{CD}^{+} 5^{+}$, respectively). Also, the three constructs demonstrated comparable functionality in terms of cytokine release (Figure 1C) and cytotoxicity (Figure 1D) upon co-culture with CD20+ JeKo-1 target cells. 
A

\begin{tabular}{l|c|l|l|l|l|} 
& aCD20_hl & IgG1 Spacer & CD8 $\alpha$ & 4-1BB & CD3z \\
\cline { 2 - 5 } CD20_hl_IgG1 & aCD20_lh & CD8 $\alpha$ Spacer & CD8 $\alpha$ & 4-1BB & CD3z \\
CD20_Ih_CD8 & aCD20_hl & CD8 $\alpha$ Spacer & CD8 $\alpha$ & 4-1BB & CD3z \\
\hline
\end{tabular}

B

do

d5

d12
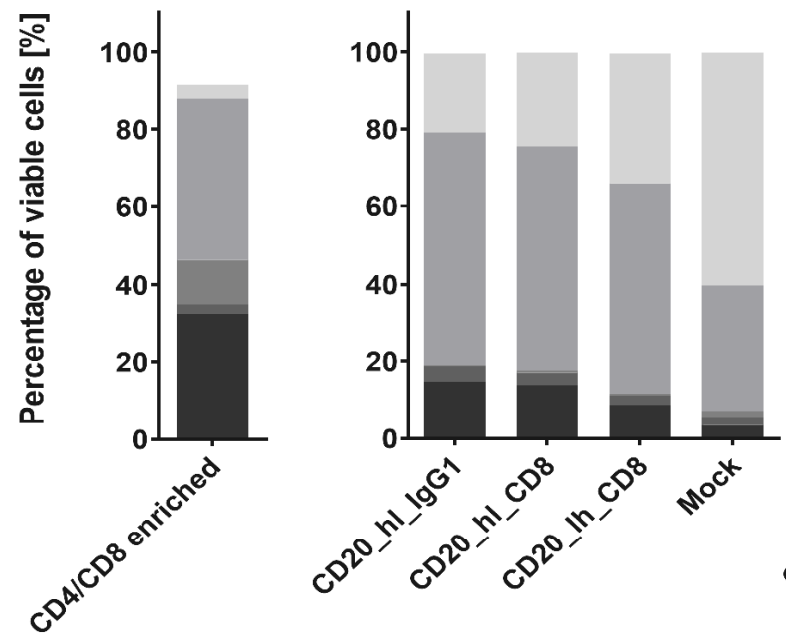

C
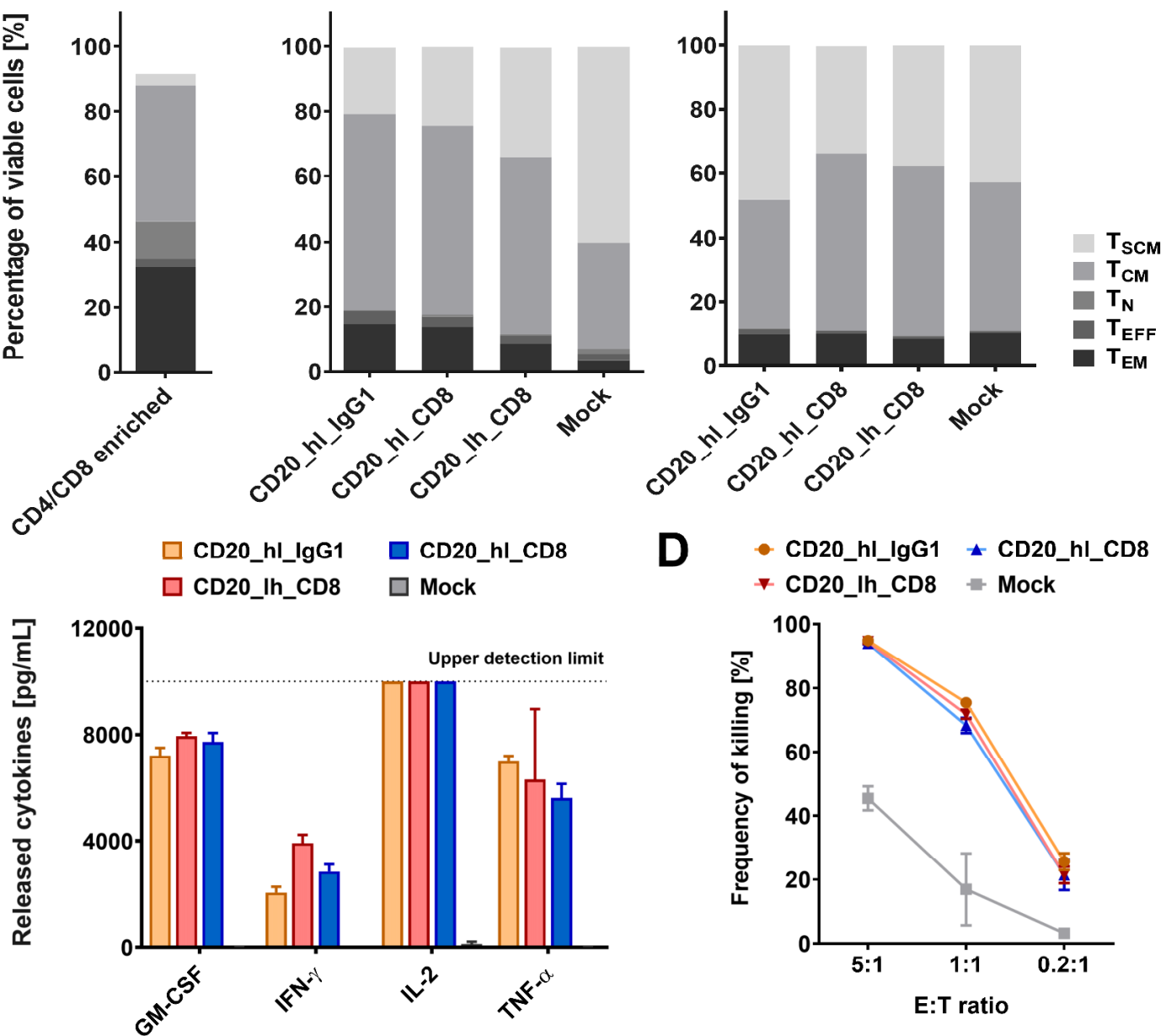
D - CD20_hl_IgG1 $\neq$ CD20_hl_CD8
$*$ CD20_lh_CD8 - Mock

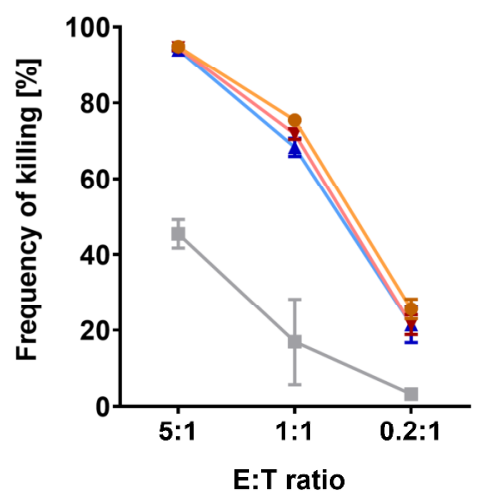

Figure 1. CD20 specific CAR T cells with short CD8 $\alpha$ and long IgG1 CH2-CH3 spacers show similar in vitro functionality. (A) Structure of the three CD20 CAR constructs. (B) T cell phenotypes in the CD4/CD8 enriched fraction on $\mathrm{d} 0, \mathrm{~d} 5$, and $\mathrm{d} 12$ of the automated T cell transduction process by flow cytometry. (C) GM-CSF, IFN- $\gamma$, IL-2 and TNF- $\alpha$ production after $24 \mathrm{~h}$ co-culture of CD20 CAR T cells with CD20 ${ }^{+}$JeKo- 1 target cells at 1:1 effector to target ratio analyzed by flow cytometry. $n=3$. (D) Cytolytic activity of the engineered CAR T cells. Effector CAR T cells and target-positive JeKo-1 target cells were co-cultured for $24 \mathrm{~h}$ at the indicated ratios (E:T). Detection of the specific lysis was performed by flow cytometry. $n=3$. 


\section{CD8 and IgG1 CH2-CH3 Spacer CARs Differ in Their in vivo Performance}

Having assessed the in vitro activity, we next analyzed the same lentivirally modified $\mathrm{T}$ cells in a pre-clinical NSG mouse model. $5 \times 10^{5} \mathrm{CD} 20^{+}$Raji cells, which were modified to constitutively express luciferase, were injected into the tail vein of each mouse. Seven days later, $1 \times 10^{6} \mathrm{CD} 20$ specific CAR T cells or GFP transduced Mock T cells (Figure 2A) were also applied intravenously. Tumor burden was monitored longitudinally over 3 weeks by noninvasive bioluminescent imaging (BLI) of tumor cells in vivo. Neither the Mock treated group nor mice treated with the IgG1 spacer CAR showed any control of tumor growth compared to the untreated group, and the animals in these groups were sacrificed according to the ethical code on day 17 and day 15, respectively (Figure 2B). On the other hand, significant therapeutic responses were achieved by the CD20_hl_CD8 and CD20_lh_CD8 CAR T cells (Figures 2B,D). Both groups exhibited a reduced tumor growth 6 days post $\mathrm{T}$ cell injection. While the CD20_hl_CD8 CAR T cells reached background fluorescence on day 13, CAR T cells equipped with the same $\mathrm{CAR}$ but with the $\mathrm{scFv}$ in the converse orientation needed longer to reduce tumor burden and did not reach background levels until the end of the experiment. This difference between the $\mathrm{scFv}$ variants could be attributed to a single mouse having remnants of tumor present in the jawbone, which in our experience is difficult to treat and possibly inaccessible to CAR $\mathrm{T}$ cells. We verified that the $\mathrm{scFv}$ orientation indeed had only a minor influence by repeating the experiment with the CD8 $\alpha$ spacer CARs with the different $\mathrm{scFv}$ orientations using a different donor (Figures 2C,E). Again, both groups of CAR-modified T cells were effective in rapidly controlling the tumor growth, with no significant difference being observed between the different $\mathrm{scFv}$ orientations. Ex vivo analysis of spleen, bone marrow and blood at the end of the study showed no detectable IgG1 spacer CAR T cells in the treated mice while CAR T cells with the CD8 $\alpha$ spacer could be readily detected, implying a reduced in vivo persistence or expansion of the T cells modified with the IgG1 spacer CAR (Figure S2). 
A

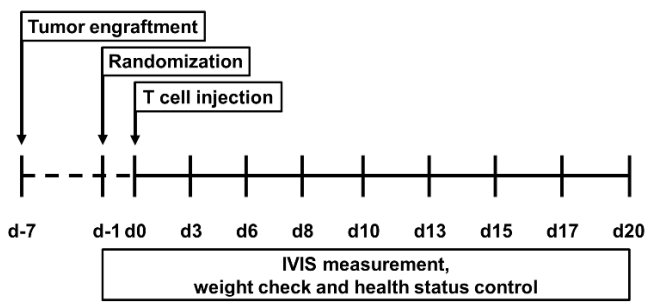

B

C
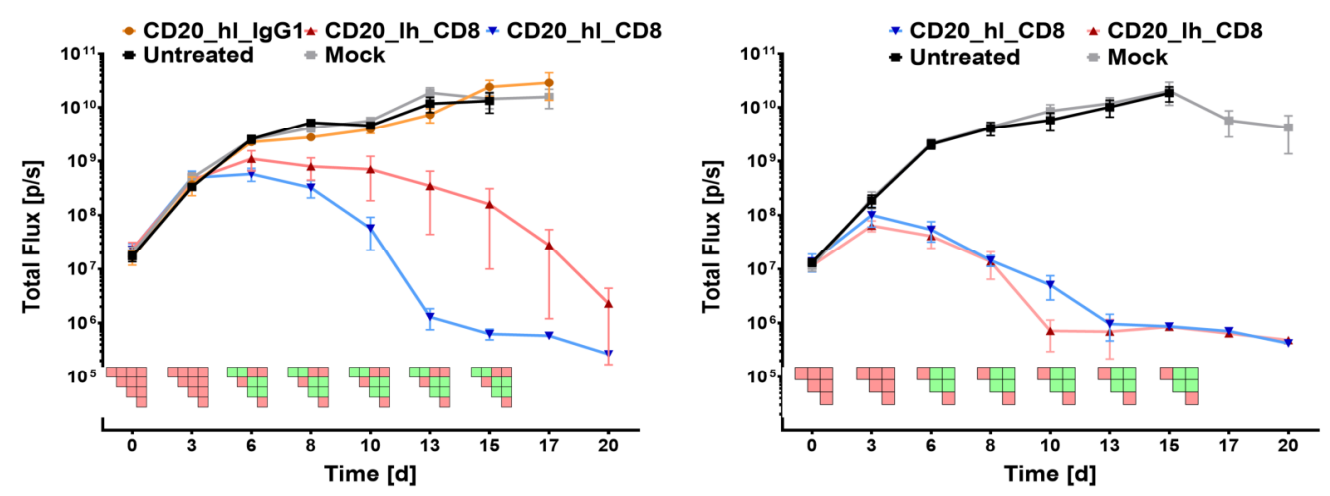

D

d6

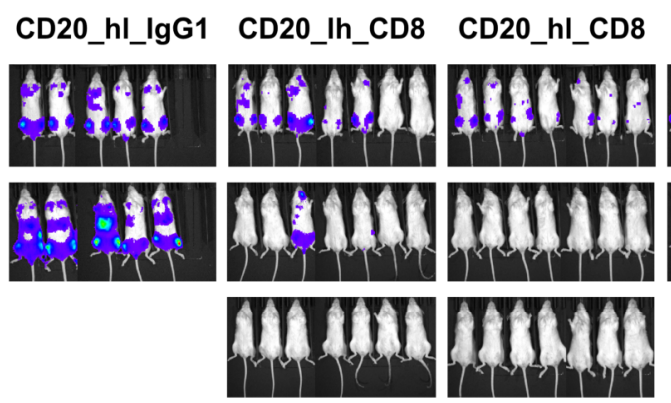

$\mathbf{E}$

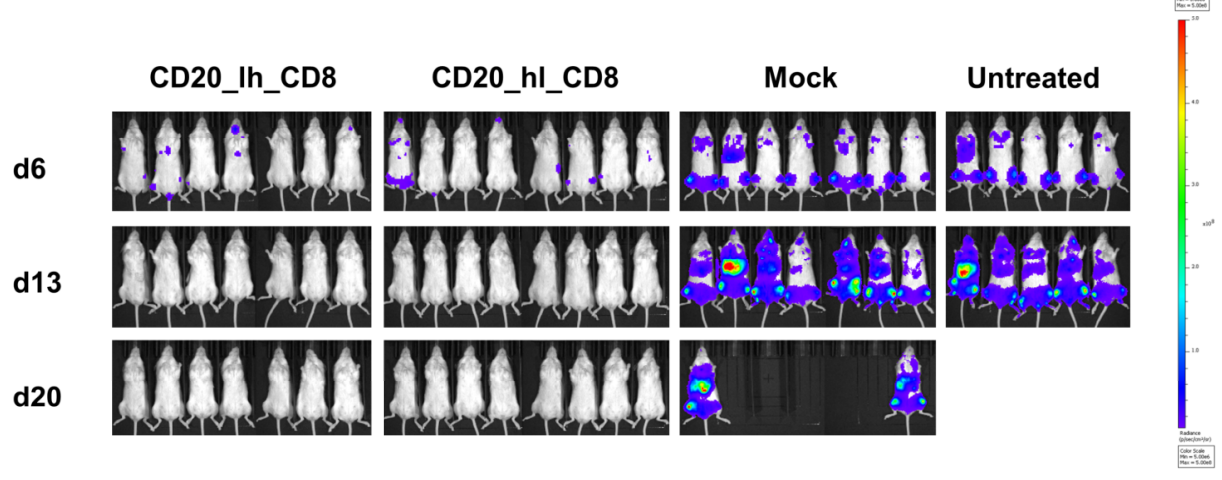

Figure 2. CD20 specific CAR T cells with an IgG1 spacer domain fail to exhibit in vivo efficacy. (A) Overview of the study workflows. (B) Tumor burden change over time in mice treated with anti-CD20 IgG1 CH2-CH3 and CD8 $\alpha$ spacer CAR T cells from one donor. $n=5$ /group. PSM $p<0.05$ (green) [one-way ANOVA]. (C) Tumor burden change over time in presence of the two different CD8 $\alpha$ CAR constructs with $\mathrm{T}$ cells from a second donor. $n=6$ /group. PSM $p<0.05$ (green) [one-way ANOVA]. (D) Representative in vivo bioluminescence images of tumor bearing mice from (B). Images are arranged according to the treatment group and time after CAR T cell injection. T cells were generated from one donor. Scale factor: min: $5 \times 10^{6}$, max: $5 \times 10^{8} \mathrm{p} / \mathrm{s}$. (E) Representative in vivo bioluminescence images of tumor bearing mice from $(\mathbf{C})$. Images are arranged according to the treatment group and time after CAR T cell injection. T cells were generated from a second donor. Scale factor: min: $5 \times 10^{6}$, $\max : 5 \times 10^{8} \mathrm{p} / \mathrm{s}$. 
These findings were in line with earlier results of other groups, showing reduced in vivo efficacy of full length IgG family spacers $(25,27)$. These groups mutated FcR binding sites or developed other solutions in order to decrease off-target binding of the T cell, which we were also able to confirm in an in vitro assay using mouse macrophages (Figure S3), but it is unclear whether all potential off-target binding has been abrogated as binding to other lower affinity Fc $\gamma$ Rs may be retained (25).

\section{Construction and Characterization of a New Family of CAR Spacers}

These findings motivated us to develop a new class of CAR spacer regions that naturally lack FcR binding sites. In this context we identified the Sialic acid-binding immunoglobulin-type lectin (Siglec) family whose members are expressed on various immune cells and incorporate Ig-like domains in their receptor architecture $(43,44)$. More specifically, while the membrane distal sialic acid binding Ig-like V-set domain is positioned N-terminally, the more C-terminally located Ig-like C2-set domains, which vary in number, serve as spacer regions. Based on previous reports describing that CAR T cell activation can be optimized according to the epitope location and spacer length, we selected one, two or three C2-set domains derived from Siglec$3,-4,-7$, or -8 for spacer design (Figure 3 ).

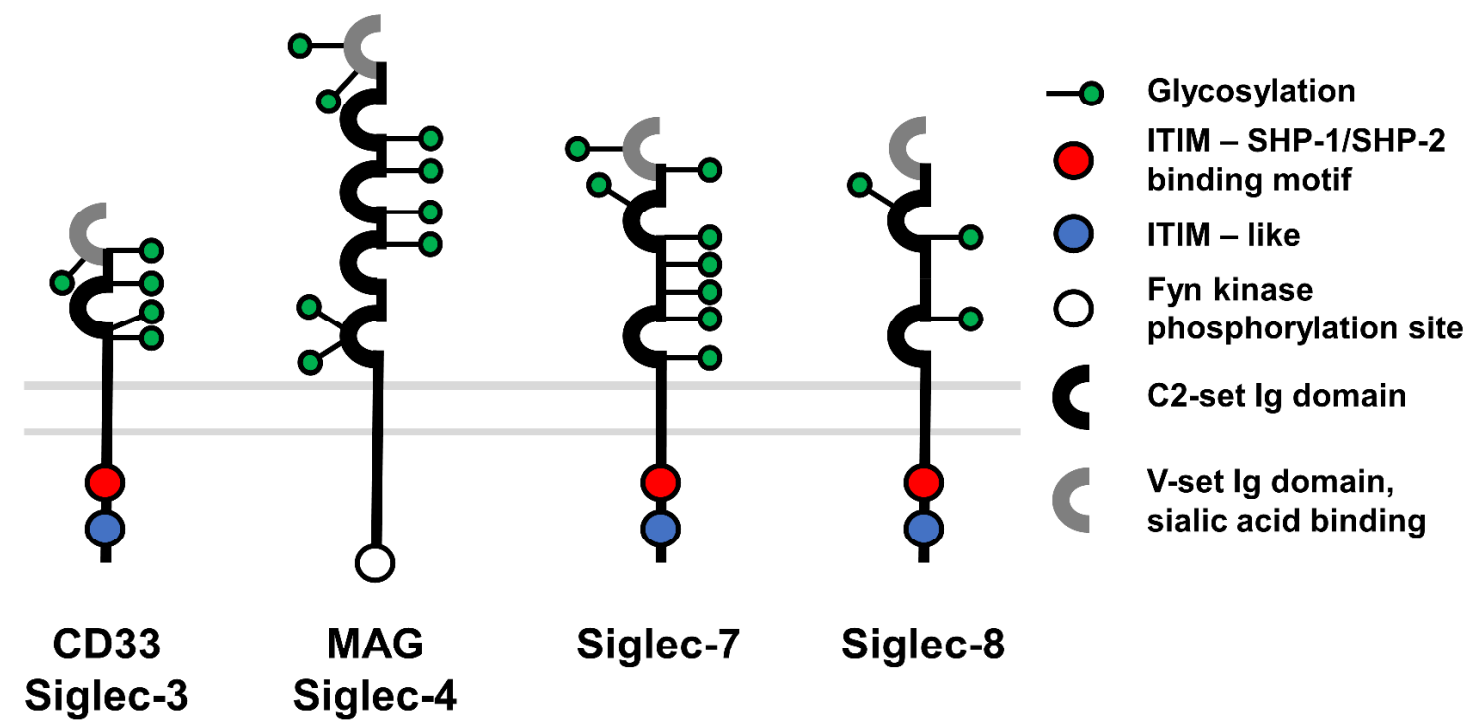

Figure 3. Overview of Siglec membrane proteins used for CAR construction. 
To confirm correct translation and surface expression of the constructs, bicistronic lentiviral expression vectors were generated with a downstream $\triangle$ LNGFR gene linked to the CAR by a P2A sequence (Figure 4A). After transfection of the DNA constructs into HEK293T cells, detection of the reporter protein $\triangle \mathrm{LNGFR}$ confirmed successful transcription and translation of the CAR cassette, while direct staining of the CAR with a CD20 CAR detection reagent (PE) visualized surface expression of the CAR constructs. All constructs showed both $\triangle$ LNGFR and CAR expression in $>80 \%$ of HEK293T cells (Figure 4B). Subsequently, we transduced primary T cells with lentiviral vectors and assessed the CAR expression 6 days post transduction (Figure $4 C)$. The $\triangle$ LNGFR reporter protein was expressed in all cases demonstrating effective lentiviral transduction of the $\mathrm{T}$ cells and translation of the expression cassette (range 46-75\% $\mathrm{LNGFR}^{+}$ T cells). However, while three CAR constructs showed CAR expression levels comparable to the CD8 $\alpha$ spacer CAR control, no CD20_hl_Sig7.1 CAR expression was detectable and the CD20_hl_Sig3 CAR was expressed on only 5\% of the T cells. Based on these results, we excluded these constructs from further analysis. 


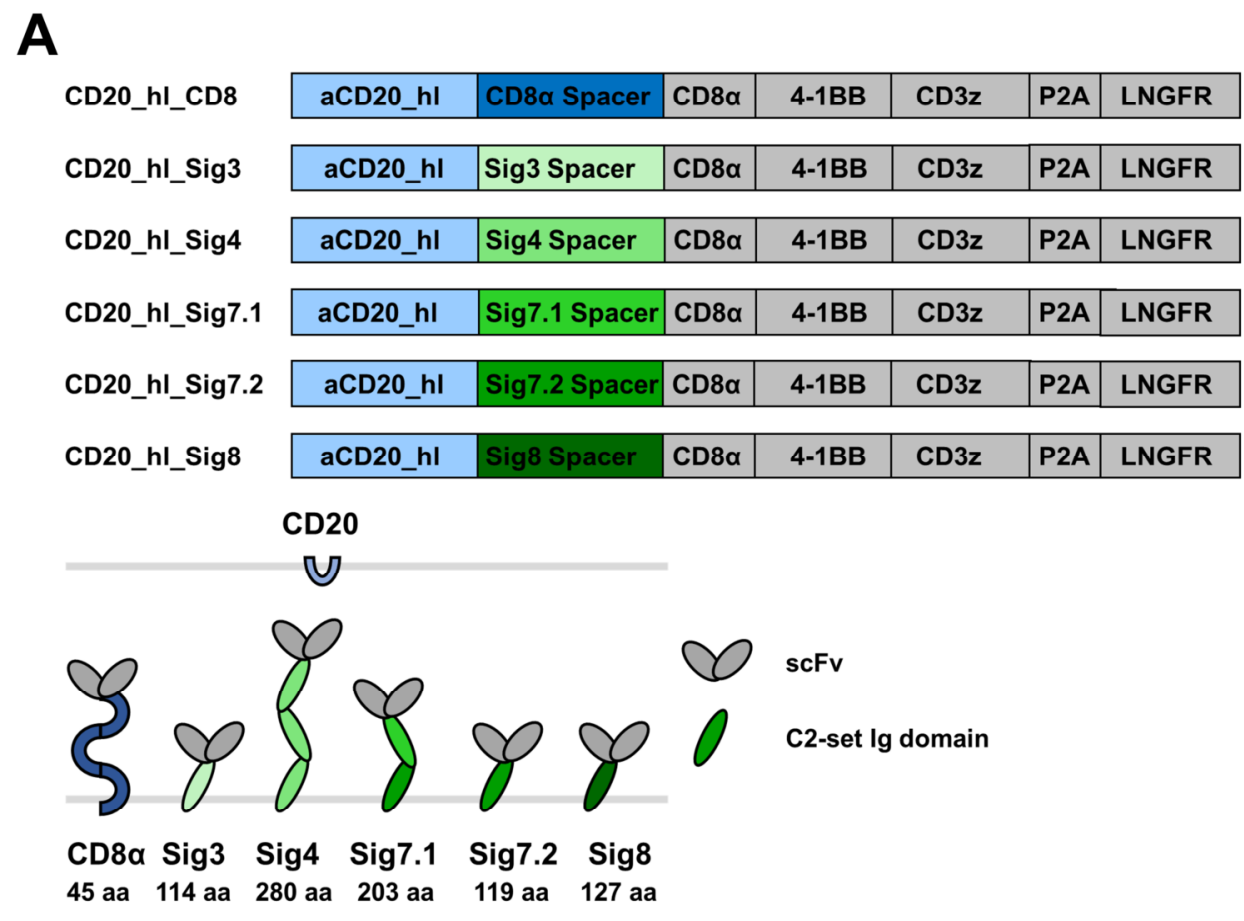

B

HEK293T

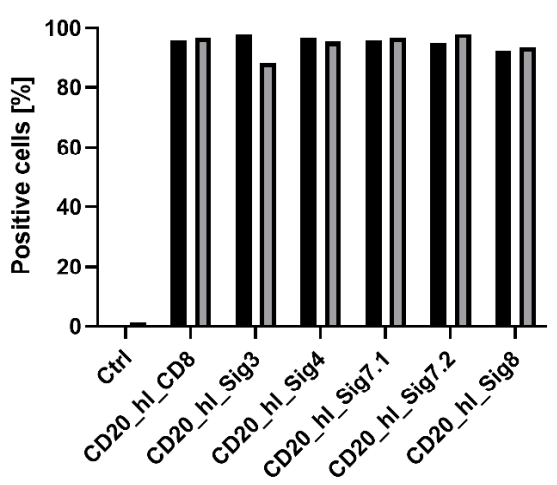

C

T cells

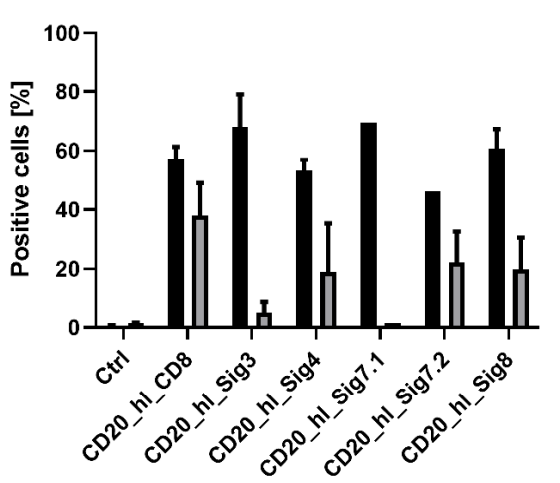

- LNGFR+

口 CAR+

D

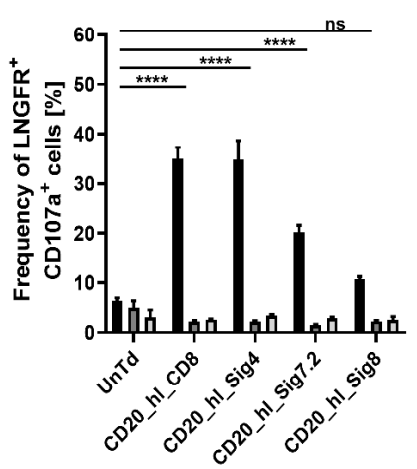

E

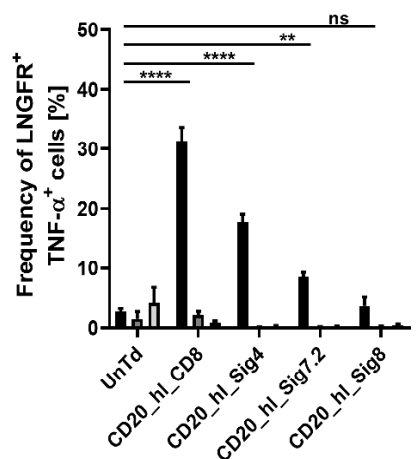

F

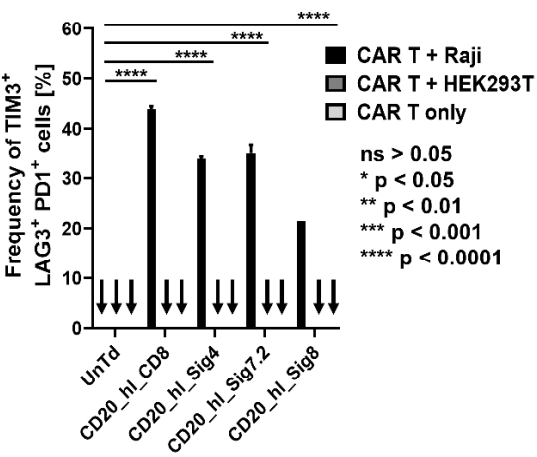

Figure 4. In vitro evaluation of novel CD20 specific Siglec spacer CAR T cells. (A) Modular structure of the CD20 CAR constructs with the Siglec spacers and extracellular domain comparison of the CAR constructs. (B) Expression analysis of the CAR constructs in transiently transfected HEK293T cells $24 \mathrm{~h}$ post transfection and (C) in transduced T cells from two donors 6 days post transduction. LNGFR and CAR expression were evaluated by flow cytometry. (D,E) Siglec spacer CAR T cells were cocultured with Raji or HEK293T cells for $5 \mathrm{~h}$ at a ratio of 1:1 and T cell expression of CD107a (D) and intracellular TNF- $\alpha(\mathbf{E})$ were analyzed by flow cytometry. (F) The frequency of TIM3, LAG3, and PD1 triple positive CAR T cells was analyzed after 24 h co-culture at a 1:2 
ratio of CAR T cells to Raji or HEK293T cells by flow cytometry. CAR T cells alone were also cultured in order to exclude unspecific activation. $n=3$. Error bars, mean \pm SD. $n s>0.05, * * p<0.01$, and $* * * * p<0.0001$ [oneway ANOVA, CAR T + Raji compared to Untreated (UnTd)].

\section{Siglec-4 Spacer Shows Comparable in vitro Functionality To CD8a Spacer in a CD20 CAR Model}

Next, we investigated the cytotoxic potential of the novel constructs. We co-cultured CAR T cells for $5 \mathrm{~h}$ with CD20+ Raji cells or CD20- HEK293T cells at an E:T ratio of 1:1. Effector function was assessed by determining degranulation and intracellular detection of the cytokine TNF- $\alpha$ in the transduced cells (gated on $\triangle$ LNGFR expression). Only CAR T cells co-cultured with CD $20^{+}$target cells showed significant degranulation (Figure 4D). Strongest degranulation could be observed for the CD8 $\alpha$ and Siglec-4 spacer variants with around 35\% of CD107 $\alpha$ positive cells. The Siglec-7.2 spacer CAR produced an intermediate amount of CD107 $\alpha$ at 20\% positive cells and the Siglec- 8 variant had the lowest degranulation with only $10 \%$ positive cells but still more than the negative controls (Figure 4D). Similar to the degranulation analysis, the proportion of $\Delta \mathrm{LNGFR}^{+} / \mathrm{TNF}^{+} \alpha^{+}$cells was also highest in CD8 $\alpha$ spacer CAR T cells (Figure 4E; 31\%) but the CD20_hl_Sig4 CARs only displayed 18\% of TNF- $\alpha$ positive cells, followed by Siglec-7.2 and Siglec-8 spacer CARs. Again, no unspecific activation could be observed in the controls.

We also assessed the activation state of the modified T cells by analyzing TIM3, LAG3, and PD1 surface expression. CD20 Raji cells were co-cultured with CAR T cells for $24 \mathrm{~h}$ at an E:T ratio of 1:2. The CD8 $\alpha$ and Siglec-4 spacer CAR modified T cells contained the largest fraction of TIM3/LAG3/PD1 triple positive cells (Figure 4F). As the Siglec-7.2 and Siglec-8 spacer CAR T cells displayed lower degranulation and upregulation of activation markers after antigen engagement throughout these in vitro experiments, we decided to investigate only the Siglec-4 spacer in more detail.

\section{The Siglec-4 Spacer CAR Displays High Functional Potency Against Membrane-Proximal Targets}

In our CD20 $0^{+}$Raji lymphoma model the Siglec-4 spacer CAR demonstrated a comparable in vitro functionality to the CD8 $\alpha$ spacer CAR. As described above the Leu16 epitope is very proximal to the target cell membrane, making it more susceptible for engagement with long spacer CARs. From the CAR variants that could be efficiently expressed in T cells, the Siglec4 spacer was the only spacer with three C2-set Ig domains, agreeing with previous work that long spacers are excellent for targeting "short," membrane-proximal targets. To verify this hypothesis and to prove the robustness of the Siglec-4 spacer functionality, we assessed the 
Siglec-4 spacer CAR in an additional solid tumor model of pancreatic ductal adenocarcinoma (PDAC). We have recently identified CD66c and TSPAN8 as novel target candidates for cellular treatment of PDAC (Schäfer et al. manuscript under revision). These two target molecules are especially suitable for investigating our novel long spacer, as the scFv binding epitopes differ greatly in terms of membrane proximity.

TSPAN8 has two extracellular loops extending from the membrane that span 24 and 96 amino acids, respectively, the larger having two interconnecting disulfide bonds. Thus, the whole protein is very membrane proximal. On the other hand, CD66c is a glycophosphatidylinositol anchored protein and consists of two C2-set domains and one V-set domain. In consequence it extends further into the extracellular space compared to TSPAN8. In addition, the epitope of the aCD66 $\mathrm{scFv}$ is localized on the outer $\mathrm{N}$ terminal $\mathrm{V}$-set domain. In summary, TSPAN8 can be considered a membrane proximal target, while CD66c is a membrane distal target.

We exchanged the Leu16 scFv from our CD20_hl_Sig4 CAR with the CD66c and TSPAN8 specific scFvs that were previously identified (Figure 5A) (Schäfer et al. manuscript under revision). Additionally, we incorporated in our experiments CD66c and TSPAN8 specific CD8 $\alpha$ spacer CARs and a TSPAN8 specific IgG4 CH2-CH3 spacer CAR, which contained a 4/2 NQ mutation in the $\mathrm{CH} 2$ domain as well as a $\mathrm{S} \rightarrow \mathrm{P}$ substitution which has been reported to reduce FcR binding also in vivo (25), which was not the case for our IgG1 construct (25). 
B

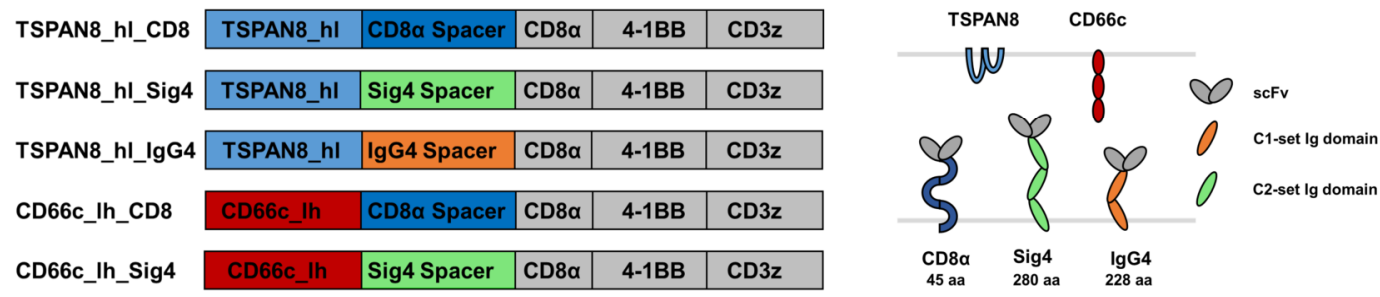
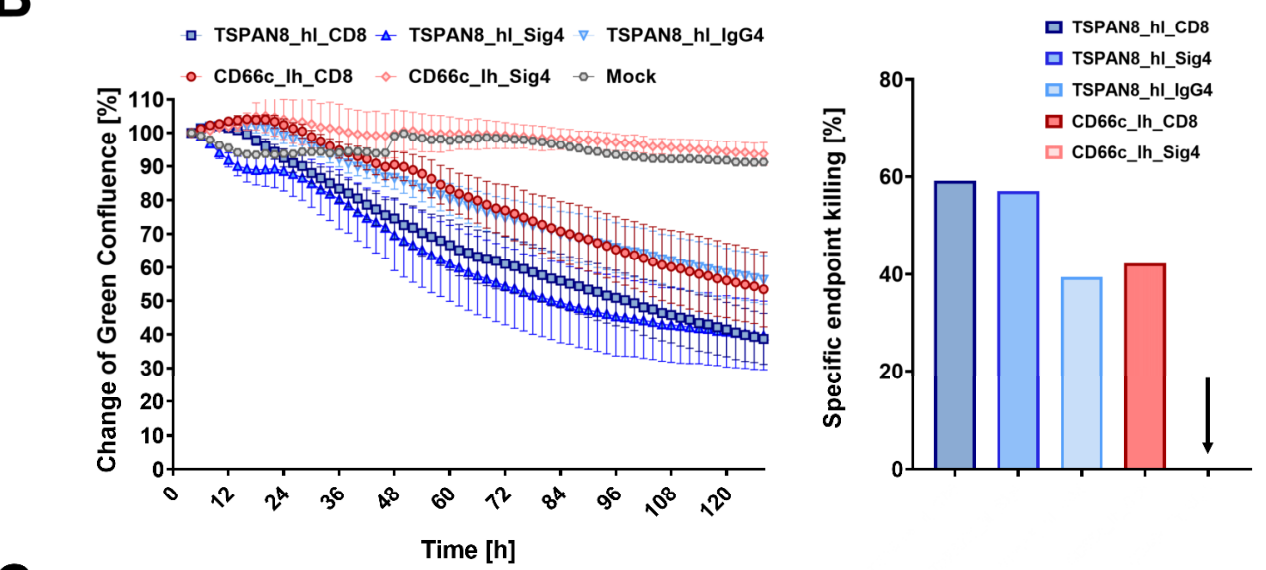

C
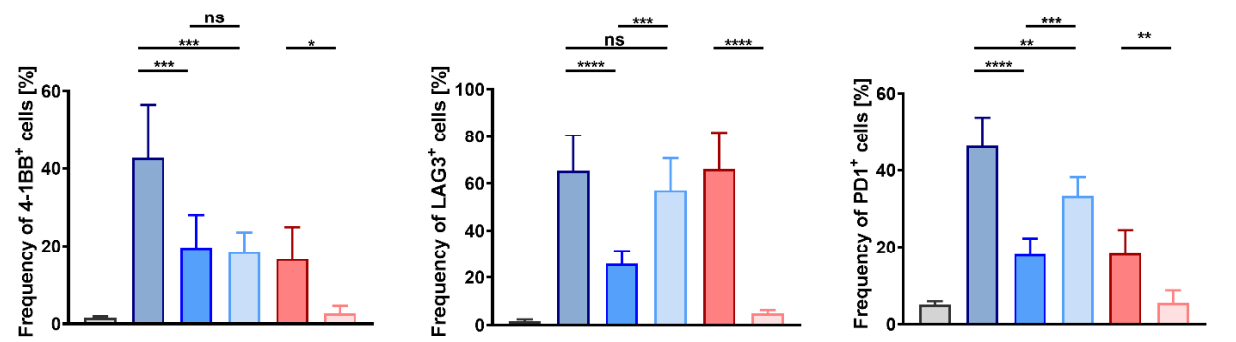

Mock

口 TSPAN8_hl_CD8

口 TSPAN8_hl_Sig4

口 TSPAN8_hl_lgG4

口 CD66c_lh_CD8

口 CD66c_Ih_Sig4

D
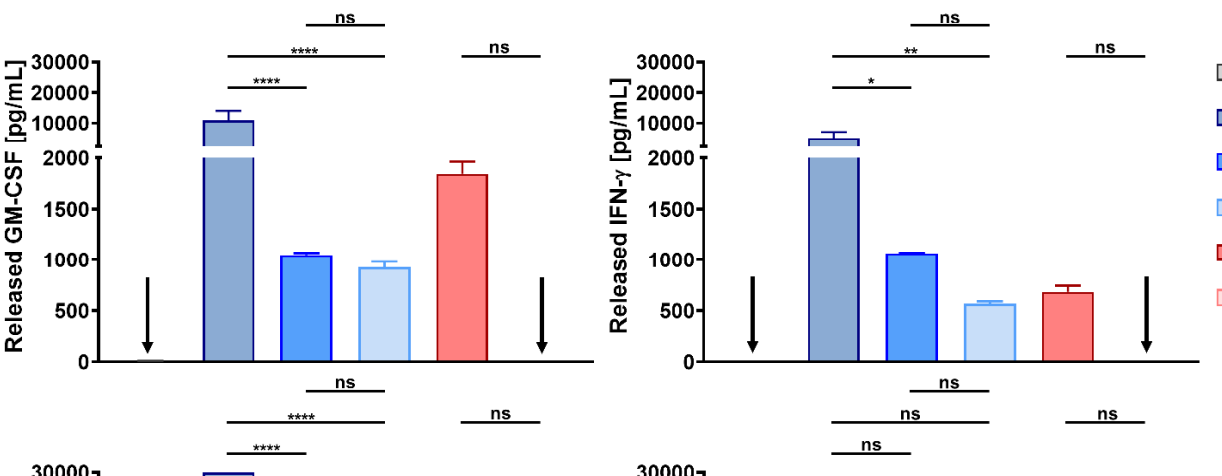

Mock

$\square$ TSPAN8_hl_CD8

$\square$ TSPAN8_hl_Sig4

$\square$ TSPAN8_hl_IgG4

$\square$ CD66c Ih CD8

$\square$ CD66c_Ih_Sig4
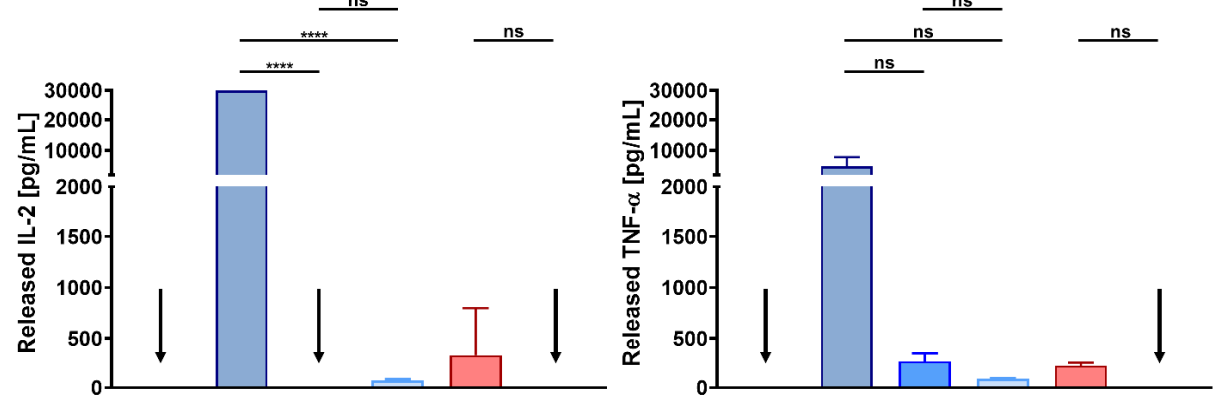

Figure 5. In vitro comparison of T cells transduced with TSPAN8 and CD66c CAR constructs, incorporating different spacer domains. (A) Structure of the TSPAN8 and CD66c CAR constructs with the Siglec spacers and extracellular domain comparison of the CAR constructs and target molecules. (B) Cytolytic kinetics and specific endpoint killing of AsPC1 target cells incubated with CAR T cells and Mock T cells from three different donors 
in effector to target ratios of 2:1. $n=6$. (C) Frequency of 4-1BB, LAG3 and PD1 positive CAR T cells was analyzed at the end of the cytolytic evaluation with AsPC1 cells by flow cytometry. (D) GM-CSF, IFN- $\gamma$, IL-2 and TNF- $\alpha$ production after $24 \mathrm{~h}$ of co-culture of TSPAN8 or CD66c CAR T cells with AsPC1 cells from one donor assessed by flow cytometry. $n=2$. Data from $(\mathbf{B}-\mathbf{D})$ were taken from the same experiment. Shown is the mean \pm SD. $n s>0.05, * p<0.05$, $* * p<0.01$, *** $p<0.001$, and $* * * * p<0.0001$ [one-way ANOVA, multiple comparisons].

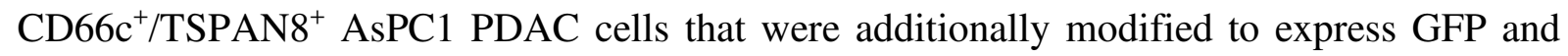
luciferase were co-cultivated with CAR T cells specific for CD66c and TSPAN8 at an E:T ratio of 2:1 and analyzed using a fluorescent live cell analysis system. We assessed cytotoxicity as a decrease in green fluorescence surface area normalized to $2 \mathrm{~h}$ after co-inoculation. After $48 \mathrm{~h}$, a supernatant sample was taken for cytokine quantitation while activation markers were measured at the end of the experiment (132 h).

Both, the CD66c_lh_Siglec-4 CAR T cells, as well as the untransduced control T cells showed no specific killing of target cells, while the CD66c_lh_CD8 CAR showed a specific endpoint killing of $42 \%$, (Figure 5B). On the other hand, when targeting the membrane proximal TSPAN8, the Siglec-4 spacer CAR $\mathrm{T}$ cells showed a similar killing to that of the TSPAN8_hl_CD8 $\alpha$ CAR T cells approaching 60\% endpoint killing. In contrast, CAR T cells modified with a TSPAN8 CAR with the alternative long IgG4 CH2-CH3 spacer exhibited only $40 \%$ killing at the end of the experiment, showing the weakest cytotoxicity of all tested TSPAN8 CAR T cells. The CD66c_lh_Sig4 CAR T cells, which showed no cytotoxicity, also expressed no activation markers (Figure 5C). The strongest upregulation of activation markers 4-1BB, LAG3 and PD-1 was observed in TSPAN8_hl_CD8 $\alpha$ CAR T cells. Interestingly, the TSPAN8 specific Siglec-4 CAR T cells displayed a lower expression of activation markers, even though the cytotoxicity equaled that of the CD8 $\alpha$ spacer CAR T cells. This difference between the CD8 $\alpha$ and the Siglec- 4 spacer CAR T cells was even more striking at the cytokine level (Figure 5D). The TSPAN8_hl_CD8 CAR T cells released markedly higher levels of cytokines than the other CAR T cells. The TSPAN8_hl_Sig4 CAR T cells secreted cytokines at levels more similar to CD66c_lh_CD8 and TSPAN8_hl_IgG4 CAR T cells, which was very surprising, with regard to the same observed cytotoxicity as the TSPAN8 CD8 $\alpha$ CAR T cells.

\section{The Siglec-4 Spacer Is Highly Efficacious in an in vivo PDAC Model}

Finally, we investigated the functionality of the three TSPAN8 specific CAR constructs in vivo in a pre-clinical PDAC tumor model. $1 \times 10^{6} \mathrm{AsPC} 1 \mathrm{eGFP} / \mathrm{Luc}^{+}$cells were injected subcutaneously in NSG mice. Tumor growth was measured non-invasively by BLI imaging and furthermore assessed by physical caliper measurement. When the first tumors reached a diameter of $25 \mathrm{~mm}^{2}$, treatment groups were randomized according to the BLI signal and tumor 
size, and treatment was started by i.v. injection of $5 \times 10^{6} \mathrm{CAR}$ T or untransduced Mock T cells (Figure 6A). Untransduced T cells did not display a therapeutic benefit over the untreated group (Figure 6B). All mice from these two groups had to be sacrificed before the end of the experiment as tumor ulcerations began to become established. The therapeutic effect for the CD8 $\alpha$ and Siglec-4 CARs became apparent in BLI measurements from day 6 onwards. The tumor burden within the groups treated with the CD8 $\alpha$ and Siglec-4 spacer CARs decreased in a comparable manner and reached baseline by the end of the experiment 29 days after $\mathrm{T}$ cell injection. At the same time, tumor growth was controlled by the $\mathrm{IgG} 4 \mathrm{CH} 2-\mathrm{CH} 3$ spacer group, but there was no tumor clearance as seen with the other groups. Persistence of CAR T cells could be demonstrated in the spleens of all CAR T cell treated groups with the highest amounts found in the CD8 $\alpha$ spacer CAR and Siglec-4 spacer CAR groups (Figure 6C). A markedly lower amount of CAR $\mathrm{T}$ cells could be recovered from the IgG4 spacer CAR group. Interestingly, when the phenotype of the human $\mathrm{T}$ cells was examined the proportion of $\mathrm{T}_{\mathrm{CM}}$ was twice as high in CD4 and CD8 CAR T cells of the Siglec-4 spacer CAR group as compared to the CD8 $\alpha$ spacer CAR T cells (Figure 6D). 
A

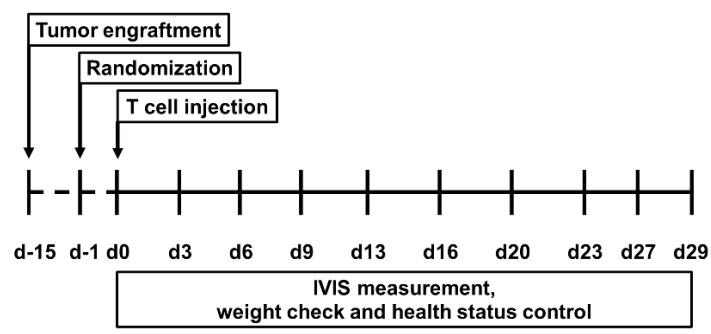

B
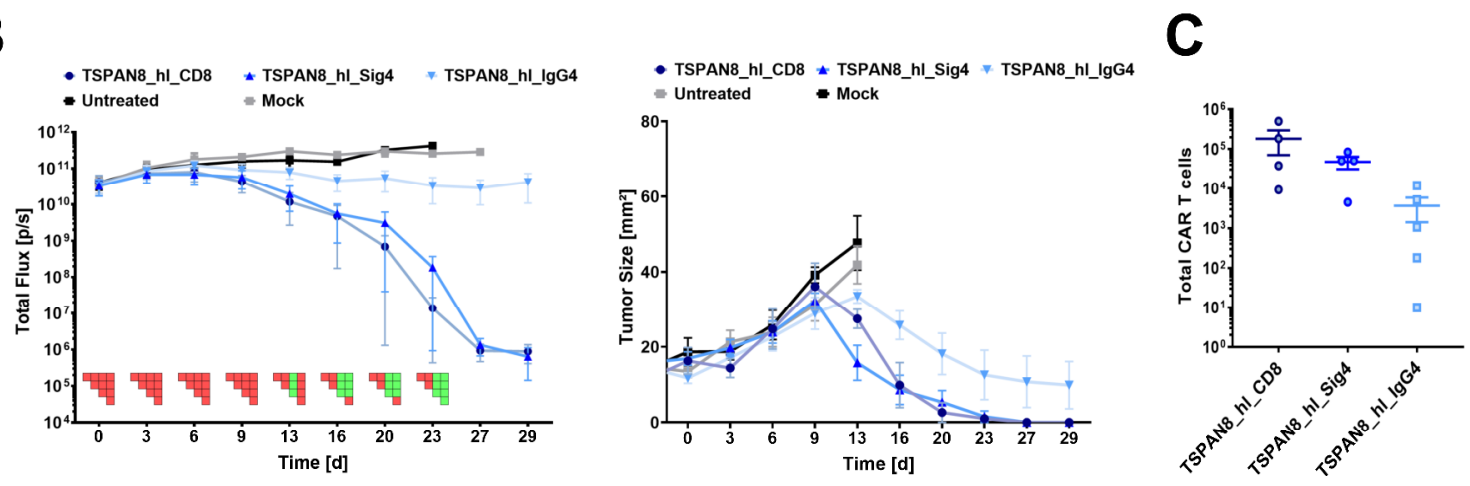

D
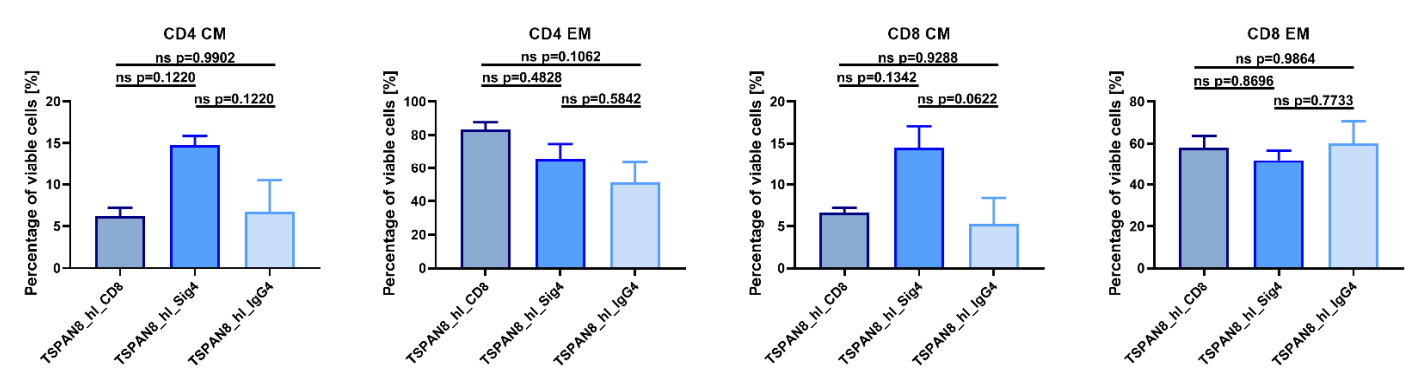

Figure 6. The TSPAN8 specific Siglec-4 spacer CAR T cells exhibit the same anti-tumor efficacy as the CD8a spacer CAR T cells, while retaining a more memory-like phenotype. (A) Overview of the study workflow. (B) Tumor burden and change in tumor size over time after TSPAN8 CAR T cell infusion. Untreated and Mock T cell treated animals served as controls, T cells from one donor were used. IgG4: $n=5$; Sig4 and CD8 $\alpha: n=4$. PSM $p<0.05$ (green) [one-way ANOVA, multiple comparisons]. (C) Total number of CAR positive T cells recovered from spleens of TSPAN8 CAR-treated animals at the end of the experiment calculated after flow cytometric analysis. IgG4: $n=5$; $\operatorname{Sig} 4$ and CD8 $\alpha: n=4$. (D) CD4 and CD8 CAR ${ }^{+} \mathrm{T}$ cell phenotypes in the spleens of TSPAN8 CAR-treated animals analyzed at the end of the experiment by flow cytometry. $n=4$.

\section{Discussion}

Despite the largely empirical design of CARs based on the functional principles of an antibody and the $\mathrm{T}$ cell receptor (TCR), CAR T cell therapies have demonstrated remarkable efficacy in the hematological tumor setting. Although a direct comparison of results across CAR T cellbased clinical trials is difficult due to the various differences in protocols, target antigens, costimulatory signaling, treatment regimen, patient groups and disease burden, the rough trend can be observed that those receptors that incorporate a CD8 $\alpha$ or CD28 spacer region in their architecture display better therapeutical efficacy than those that utilize IgG-based $\mathrm{Fc}$ domains 
(1-7, 28-31). Non-clinical studies investigating this effect suggest that the inferiority of IgG spacers is due to the engagement with Fc $\gamma \mathrm{R}$-expressing myeloid cells (23) resulting in off-target activation of both gene-modified $\mathrm{T}$ cells and the respective $\mathrm{Fc} \gamma \mathrm{R}^{+}$cells. In parallel, additional work has been demonstrating that the exemplary performance of CD8 $\alpha$ or CD28 spacer CARs is partially also attributed to the epitope location on the targeted antigen CD19 and a number of studies have affirmed the postulate that membrane-proximal epitopes are best targeted by long spacer modules while membrane-distal epitopes are effectively recognized by CARs incorporating short spacer elements $(18-22,45)$.

In light of these developments, we identified a shortage of functional CAR spacer modules for membrane-proximal epitopes. Taking advantage of the well-described CD20 antigen and the membrane-proximal binding epitope of Leu16-derived anti-CD20 scFv (42), we sought to characterize the properties of CD8 $\alpha$ - vs. IgG-based spacer CARs against CD20 in vivo. To avoid unintended cross-activation of CAR-and Fc $\gamma$ R-expressing cells in the context of the IgG spacer, the amino acid sequence for IgG1-Fc $\gamma \mathrm{R}$ interactions in the IgG1-CH2 extracellular domain of the CAR was replaced by the corresponding $\operatorname{IgG} 2$ amino acids as described previously (23). However, contrary to reports describing increased anti-tumor activity and CAR $\mathrm{T}$ cell persistence following modifications in the IgG4 spacer to abrogate Fc $\gamma \mathrm{R}$-binding in the CAR spacer domain $(25,27)$, we did not observe any in vivo therapeutic efficacy of IgG1 CAR $\mathrm{T}$ cells after similar modifications in our study. More specifically, the lack of efficacy was accompanied with an inefficient persistence of the gene-modified T cells. These results were in stark contrast to the functional capacity of the CD8 $\alpha$ CAR T cells which - according to current understanding - display a less favorable receptor architecture due to the short spacer region. Although it is reasonable to conclude that the introduced mutations into the IgG spacer domain may not entirely abrogate Fc $\gamma \mathrm{R}$ binding, it cannot be ruled out that additional mechanisms are in play that sacrifice the therapeutic efficacy. For example, it has already been described that murine scFvs and other non-self gene products can elicit HLA-restricted $\mathrm{T}$ cell-mediated immune responses $(3,46,47)$. Thus, the possibility exists that the introduced mutations into the Fc region can create immunogenic peptides by the T cell's antigen processing machinery which are then presented on the T cell's HLA and render the gene-modified lymphocytes susceptible to TCR-triggered fratricidal activity. Therefore, it is to be appreciated that the interplay of CAR $\mathrm{T}$ cells with their cognate counterparts and the immune system is complex and further work is required to understand the full immunogenic potential of CAR molecules. 
To exclude the possibility of potential immunological barriers elicited by the spacer region, we switched our test system to the IgG4 backbone which was previously described to show in vivo performance $(25,27)$ and which has also shown successful translation to the clinic $(34)$. In addition, a new set of spacer domains was designed based on the Siglec family whose members are expressed throughout the immune system and display evolutionary structural similarities to the constant region of immunoglobulins, but lack the inherent ability to interact with Fc $\gamma$ Rs (35, 36). To determine systematically the optimal spacer length for the membrane-proximal CD20 epitope, five Siglec spacer CAR variants were generated incorporating either one, two or three Ig domains. Of note, different parent proteins were selected, as different Siglec molecules encompass distinct glycosylation patterns which are likely involved in modulating the protein's stability, flexibility, spatial architecture etc. and thus may have different effects on the CAR molecule. Moreover, in an attempt to maintain the original architecture of the molecule, the domains closest to the plasma membrane were selected. Consequently, the Siglec spacer regions within the otherwise identical CAR framework encompassed either a 114 amino acid (aa) Siglec-3, 119 aa Siglec-7, 127 aa Siglec-8, 203 aa Siglec-7, and 280 aa Siglec-4 spacer domain as opposed to the control 45 aa CD8 $\alpha$ spacer domain.

Subsequent expression profiling revealed that not all Siglec spacer-based CARs were efficiently expressed on the T cell surface. In particular, Siglec-7.1 and Siglec-3 spacer CARs showed the lowest expression efficiency emphasizing the importance of the spacer region not only on the receptor's functionality but also on its optimal expression. In fact, Patel and colleagues have already described that the CAR spacer domain can affect the receptor's stability and modify its turnover rate (17). It is plausible that the glycosylation patterns present in Siglec-7.1 and Siglec3 spacer CARs render the receptors less stable, in this way increasing the turnover kinetics and a decreased CAR detectability on the cell surface. Another potential reason for the inefficient expression of the Siglec-3 spacer CAR may lie in the C169S mutation which was introduced in order to abrogate unspecific disulfide bond formation as C169 is involved in an interdomain disulfide bond within the parent protein. Moreover, it is possible that the Siglec-3 C2-set domain per-se is instable when isolated from the membrane-distal V-set domain. Although a splice variant of CD33 has been described, which lacks the N-terminal domain (CD33 ${ }^{\triangle E 2}$ ), these reports rely on mRNA analyses $(48,49)$. Protein-based detection using antibodies remains controversial, as it is still not clear whether a clone exists that can specifically recognize the Siglec-3 C2-set domain $(49,50)$. Importantly, using lentiviral transduction of His-tagged $\mathrm{CD} 33^{\triangle E 2}$, Laszlo and colleagues have shown that the expression of the splice variant is also dependent on the cell type (49). In this context, HEK293T exhibited highest transgene 
expression while hematopoietic cells displayed only low level expression of the truncated immune receptor which is in line with our observations on the expression of the Siglec-3 spacer CAR (Figures 4B,C).

In the next series of experiments, the three best expressed Siglec spacer CAR candidates were analyzed for their ability to induce T cell effector function upon antigen engagement. Consistent with previous reports $(17-19,25,26,51)$, our study provides evidence that the CAR spacer region can modulate the effector function of transgenic $\mathrm{T}$ cells. Intriguingly, however, we find that depending on the effector function analyzed, the functional hierarchy may vary. In particular with regard to cytotoxicity, no significant differences between the CD8 $\alpha$ spacer (45 aa in length, no Ig domain) and Siglec-4 spacer (280 aa in length; three Ig domains) CAR can be observed while in terms of cytokine secretion the CD8 $\alpha$ spacer CAR displays a significant dominance over other CAR constructs. Importantly, in addition to the CD20 system, this observation was further confirmed in the setting of another membrane-proximal antigen, TSPAN8, indicating a common functional feature for membrane-adjacent epitopes.

It has already been demonstrated in the TCR-context that distinct thresholds exist for the cytolytic machinery, the proliferative induction as well as the cytokine production system (5256) and emerging work suggests similar principles for CAR-triggered T cells (26). The current study further supports this finding and the data obtained indicate that the nature of the spacer region can modulate the nature and degree of effector function. An alternative strategy has been described by Liu and colleagues (57) and Caruso and colleagues (58) in two independent studies, in which they demonstrate the ability of effector function fine-tuning through $\mathrm{scFv}$ affinity modulation. The clinical impact of such modifications was impressively demonstrated by Ghorashian and colleagues, who reported a better overall therapeutic profile of CD19 CAR $\mathrm{T}$ cell therapies in patients who received lower affinity CARs compared to the commonly used FMC63-scFv-based CARs (59). In particular, while the antileukemic activity was retained, the CAR T cells displayed an enhanced proliferative capacity and reduced severity of cytokine release syndrome (CRS). Though this clearly reveals the effectiveness of such an approach, $\mathrm{scFv}$ affinity modulation is a laborious undertaking and bears the risk to result in unwanted modifications to the target specificity. Therefore, fine tuning the chimeric receptor's spacer region provides a time-profitable option with a lower risk profile. More importantly, it further allows to create a variety of receptors with a range of signal transduction intensities independent of the binding domain. 
Besides, based on the efficacy data obtained with the CD8 $\alpha$ spacer (45 aa) vs. Siglec- 4 spacer (280 aa) CARs targeting CD20 and TSPAN8, we find that the receptors' cytotoxic efficacy is not dominated by the spatial constraints of the CAR and its target epitope. This is significant as previous studies reporting such a trend were performed primarily in the context of IgG-derived sequences $(25,26,51)$ and have not been compared extensively to spacers derived from other parental proteins. Thus, our work demonstrates that not only structural and spatial elements in CAR T cell:target cell interaction influence a receptor's bioactivity, but also additional factors are in play that are not entirely understood or fully considered yet. It is likely that e.g., CAR flexibility/rigidity and surface stability may have a greater relevance than previously assumed. For instance, Patel and colleagues have shown that the spacer domain can diminish a CAR's functionality by increasing its turnover rate (17). Thus, it is important to take into consideration that Ig domains as they are present in IgG and Siglec spacer domains display a distinct structural folding while the CD8 $\alpha$ spacer is derived from a stalk connecting an Ig-like domain with the membrane. Attempts to resolve the structure of the CD8 $\alpha$ hinge domain were of limited success so far, indicating the relative flexibility of this region (60). The Siglec- 4 or the IgG spacers are missing this flexibility and in this way reduce targetable epitopes to the ones located in membrane proximity.

Another important aspect to be taken into consideration is the tendency of the CD8 $\alpha$ stalk region to heterodimerize with $\mathrm{CD} 8 \beta$, the subunit that contains raft-localizing determinants (61). As lipid rafts contain an accumulation of accessory molecules decisive for signal transduction and the intracellular CD8 $\beta$ domain has been described to promote association with the two crucial players Lck and LAT (62), it is likely that - in the context of cytotoxic T cells - the CD8 $\alpha$ spacer region is capable of attenuating the effector function threshold by fostering interaction with downstream signaling molecules. These effects are absent in IgG- and Siglec-based spacers, so that the overall induction of $\mathrm{T}$ cell function is likely primarily guided by the number of triggered CAR molecules (Figure S5).

In support of the in vitro data, the Siglec-4 spacer CAR displayed a similar anti-tumor efficacy in vivo as the cognate $\mathrm{CD} 8 \alpha$ spacer CAR against TSPAN8 and both therapies were superior to the IgG4-based spacer CAR treatment. Taking into account the length of the spacer regions (CD8a: 45aa; Siglec-4: 280 aa; IgG4: 228aa), we could not observe any obvious correlation with CAR potency and rather identified an intrinsic inferiority of the IgG4 spacer in vivo. However, in the context of the TSPAN8 targeting, the modified IgG4 spacer CAR showed a much better relative in vivo performance to the $\mathrm{CD} 8 \alpha$ spacer compared to the $\operatorname{IgG} 1$ spacer 
performance in the CD20 study. Indeed, the modified IgG4 spacer (25) has now demonstrated good efficacy in ongoing clinical studies (34) indicating that other factors in CAR design such as the $\mathrm{scFv}$ binding domain, transmembrane domain or the drug product formulation may also play a role in in vivo function and $\mathrm{T}$ cell persistence.

Strikingly, however, while the cytotoxic activity was comparable between the CD8 $\alpha$ and Siglec-4 spacer CARs, we observed a reduced secretion of pro-inflammatory cytokines and an attenuated upregulation of activation/exhaustion markers such as 4-1BB, LAG3, and PD1 in the Siglec-4 spacer CAR T cells. Moreover, while the proliferative capacity of Siglec-4 CAR T cells was slightly lower compared to CD $8 \alpha$ spacer CAR T cells, the Siglec-4 CAR-treated mice featured a trend toward a higher fraction of $\mathrm{T}_{\mathrm{CM}}$ phenotype within the CAR T cell cohort which is associated with better overall remission and decreased likelihood of relapse in a clinical context.

It is currently widely established that CAR efficacy correlates closely with the development and severity of CRS in the clinic, an adverse event whose management has proven challenging in the clinical setting. Although tocilizumab and glucocorticoids have been described as effective intervention options, finding the right timing for their application represents a big hurdle $(1,63)$. In fact, too early intervention may jeopardize the therapeutic efficacy and increase the risk of relapse, while too late intervention bears the risk of CRS-induced multiorgan failure and irreversible neurotoxicities resulting in a patient's death (1, 3, 5, 7, 64-68). Thus, a treatment modality that retains the cytotoxic ability of currently approved CAR $\mathrm{T}$ cell therapies but attenuates the levels of secreted cytokines may turn engineered $\mathrm{T}$ cells not only into a reliable and effective, but also a safer platform. Moreover, a concomitant increase of the memory phenotype in the CAR T cell cohort of the patient holds promise to further increase the therapeutic efficacy while reducing life-threatening side effects.

Although the phenotype of Siglec-4 CAR modified T cells bodes well for future clinical application, what is the potential toxicity profile of this novel spacer structure? The parent protein Siglec-4, also known as myelin-associated glycoprotein (MAG), has been reported to be exclusively produced by myelinating glial cells such as oligodendrocytes in the central nervous system (CNS; $1 \%$ of total protein mass) and Schwann cells in the peripheral nervous system (PNS; $0.1 \%$ of total protein mass) $(69,70)$. Its specific expression on the innermost layer of myelin directly opposite to the axon surface supports its crucial role in the stabilization of axon-myelin interactions, the regulation of myelination, and the inhibition of axon regeneration after injury (71-73). These effects have been first described to be mediated by the 
$\mathrm{N}$-terminal V-set domain of the receptor, as determined by ligand specificity analyses, sitedirected mutagenesis and analogy to the crystal structure of Siglec-1 (74-77). In our evaluation of homology studies, we found the protein sequence to be the best conserved among the Siglecs and within mammalian species. Indeed, the highest sequence homology was identified to lie within the first two N-terminal domains of Siglec-4 (78). Consequently, in order to abrogate these interactions, both $\mathrm{N}$-terminal domains were excluded from our CAR spacer design.

More recent studies, however, suggest that an alternative binding domain exists that interacts with the Nogo receptor 1 and 2 (NgR1, NgR2), but not NgR3 (79-82). Deletion analysis demonstrated that while the first three Ig-like C2-set domains (amino acids 17-325) of Siglec4 are involved in these interactions, C2-set domains 3-5 (amino acids 234-506) as they are present in our CAR architecture fail to associate with $\mathrm{NgR} 1$ or $\mathrm{NgR} 2$ (83) indicating that domains 1 and 2 are the major interaction partners. Interestingly, both a soluble and membranebound receptor construct comprising the C2-set domains 3-5 of Siglec-4 (amino acids 234-506) are still able to inhibit neurite outgrowth in the CNS, suggesting the existence of an as of yet unidentified ligand partner $(83,84)$. This observation may indicate the potential risk of unwanted interactions of the Siglec-4 spacer-based CAR T cells with this unknown binding partner. Although the CNS is an immune-privileged organ an intensive infiltration by CAR T cells has been shown to occur as a result of blood-brain-barrier (BBB) damage due to strong CRS. However, as Siglec-4 spacer CAR T cells appear to produce lower levels of cytokines, BBB disruption is expected to be mitigated, in this way minimizing CNS accessibility for CAR T cells.

In support of this hypothesis, despite the high homology between human and rodent Siglec-4 of $95 \%$ at the amino acid level over the entire extracellular domain $(85,86)$, we did not observe any toxicities in the mouse cohort receiving Siglec-4 spacer CAR therapy in our in vivo studies. Nevertheless, since - to the best of our knowledge - human-mouse cross-reactivity of Siglec-4 and its interaction partners has not been determined, these data need to be handled with care and further analysis is required to investigate the extent of potential side-effects of Siglec-4 spacer-based CAR T cells.

In summary, this study introduces the new class of Siglec CAR spacers, which structurally resemble IgG class spacers without their Fc $\gamma \mathrm{R}$ binding features. The Siglec-4 spacer proved to be as efficient as a conventional CD8 $\alpha$ spacer in both in vitro and in vivo CAR function, but exhibited advantageous traits in terms of the T cell phenotype and CAR T cell cytokine release, 
which make it an interesting candidate CAR structure to translate into future clinical applications.

\section{Data Availability Statement}

All datasets generated for this study are included in the article/Supplementary Material.

\section{Ethics Statement}

The studies involving human participants were reviewed and approved by German Red Cross Dortmund, University Hospital Cologne and German Red Cross Ulm. The patients/participants provided their written informed consent to participate in this study. The animal study was reviewed and approved by Landesamt für Natur, Umwelt, and Verbraucherschutz NRW, Approval numbers 84-02.04.2015.A168 and 84-02.04.2017.A021.

\section{Author Contributions}

RP designed the Siglec based spacers. DS and RP designed the Siglec spacer-based CARs. DS, JH, RP, WA, IJ, and OH designed the study. DS, JH, AS, JB, CB, DG, and WA conducted experiments. DS, JH, and RP wrote the manuscript with input from all authors. NM-T, RP, WA, $\mathrm{IJ}$, and $\mathrm{OH}$ supervised the project. All authors discussed the data and reviewed the manuscript.

\section{Conflict of Interest}

DS, JH, RP, JB, NM-T, CB, DG, WA, IJ, and OH are employees of Miltenyi Biotec B.V. \& Co. KG. Patent applications are pending to this work.

The remaining author declares that the research was conducted in the absence of any commercial or financial relationships that could be construed as a potential conflict of interest.

\section{Acknowledgments}

We would like to acknowledge Andrew Kaiser and Dina Schneider for helpful discussions, Dina Schneider for providing the CD8 CAR construct, Hinrich Abken for providing the IgG1 CAR construct, and Dominik Lock for the eGFP/ffLuc transfer vector plasmid.

\section{Supplementary Material}

The Supplementary Material for this article can be found online at: https://www.frontiersin.org/articles/10.3389/fimmu.2020.01704/full\#supplementary-material 


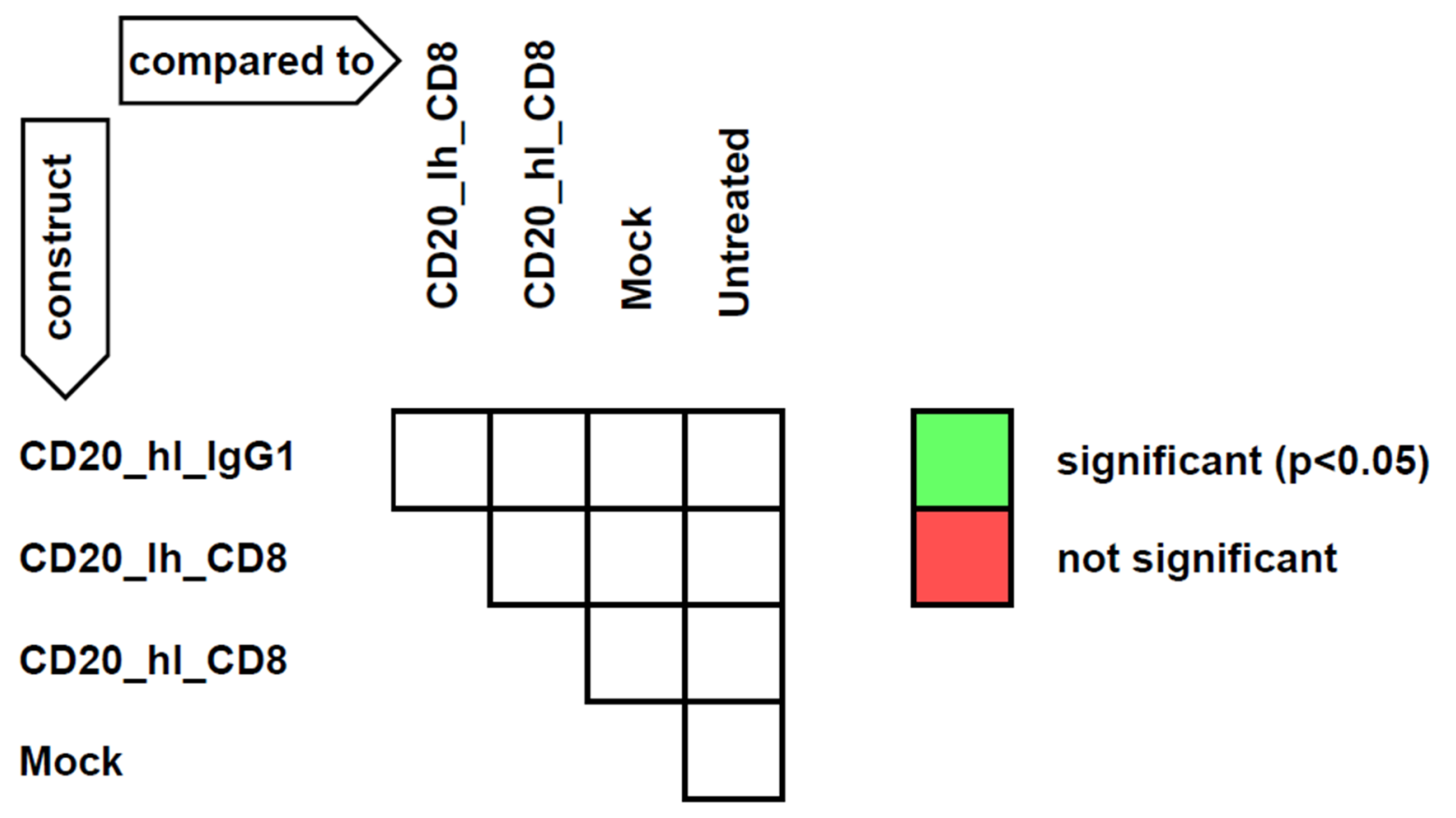

Figure S1: Organization of the pairwise significant matrix for group comparison of in vivo performance of CD20_hl_lgG1, CD20_lh_CD8, CD20_hl_CD8, Untreated and Mock. PSM p $<0.05$ (green) [one-way ANOVA, multiple comparisons]. 


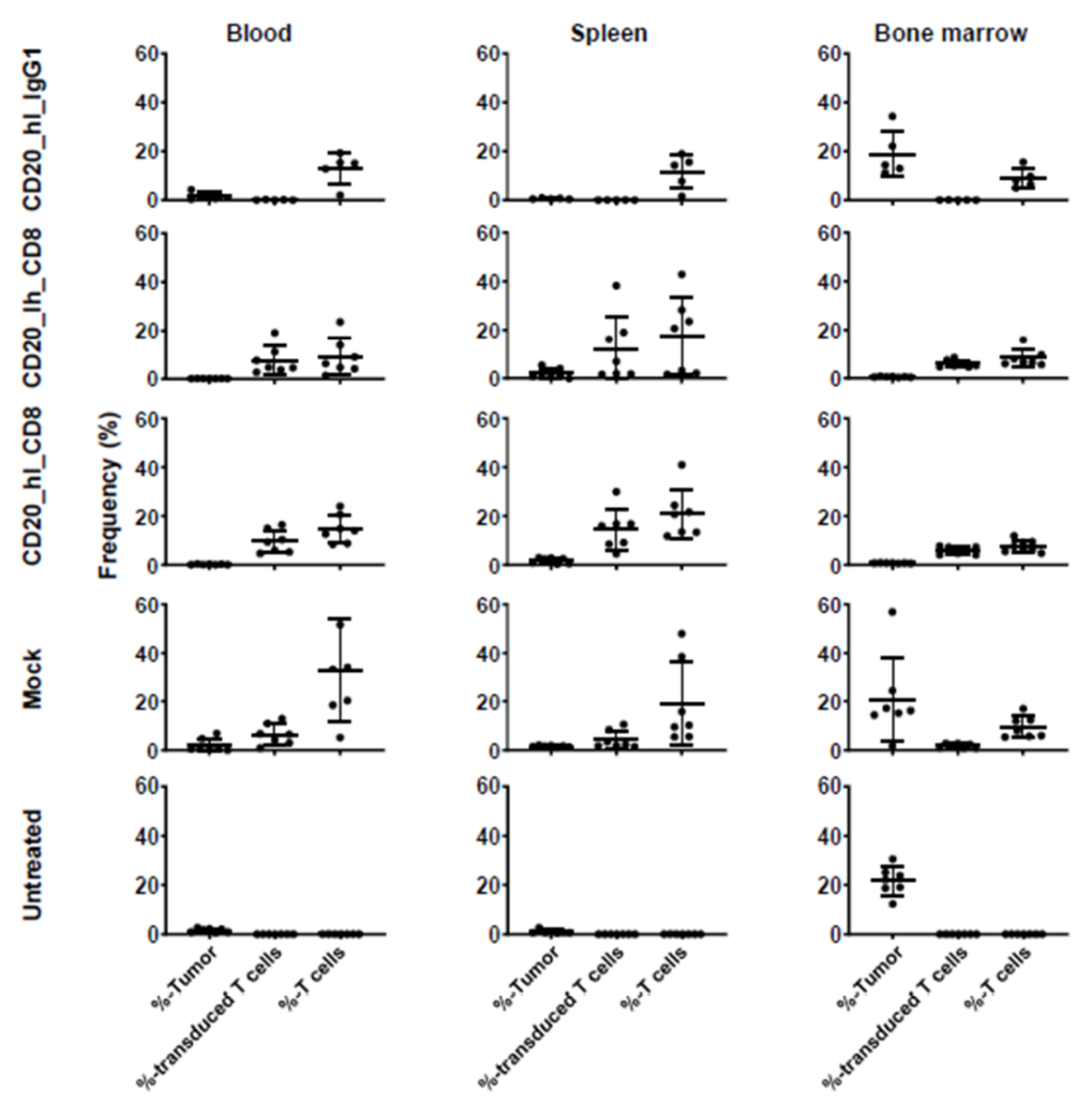

Figure S2: Flow cytometry analysis of tumor and CD20 CAR T cells persistence in blood, spleen and bone marrow. Ex vivo analyses were performed at the end point of the study. Percentage of transduced T cells in the Mock group was calculated based on GFP expression. $n=5-6$. 


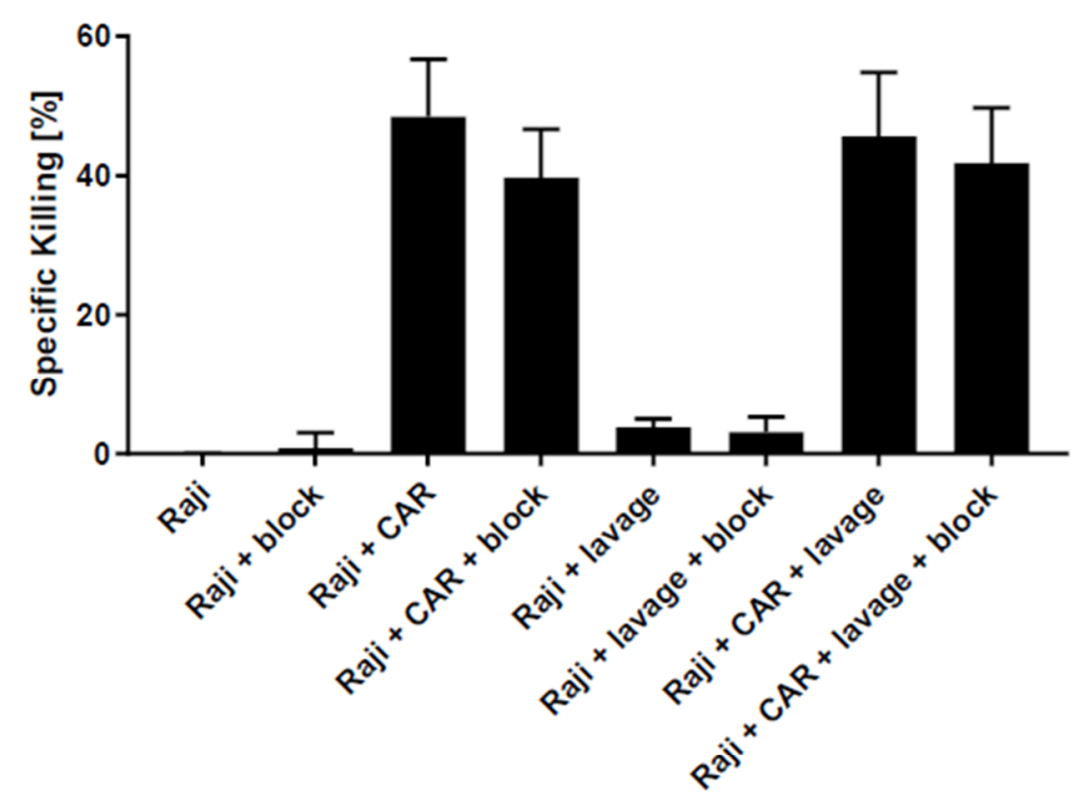

Figure S3: Macrophages do not inhibit the function of CAR T cells containing an IgG1 spacer with mutated Fc-binding sites. CD20_hl_IgG1 CAR T cells were mixed in a 1:1:1 ratio with Raji target cells and NSG macrophages, derived from a peritoneal lavage. Macrophages, tumor and CAR T cells were co-incubated for 24 hours in absence or presence of murine FcR-blocking reagent (block). $n=3$.

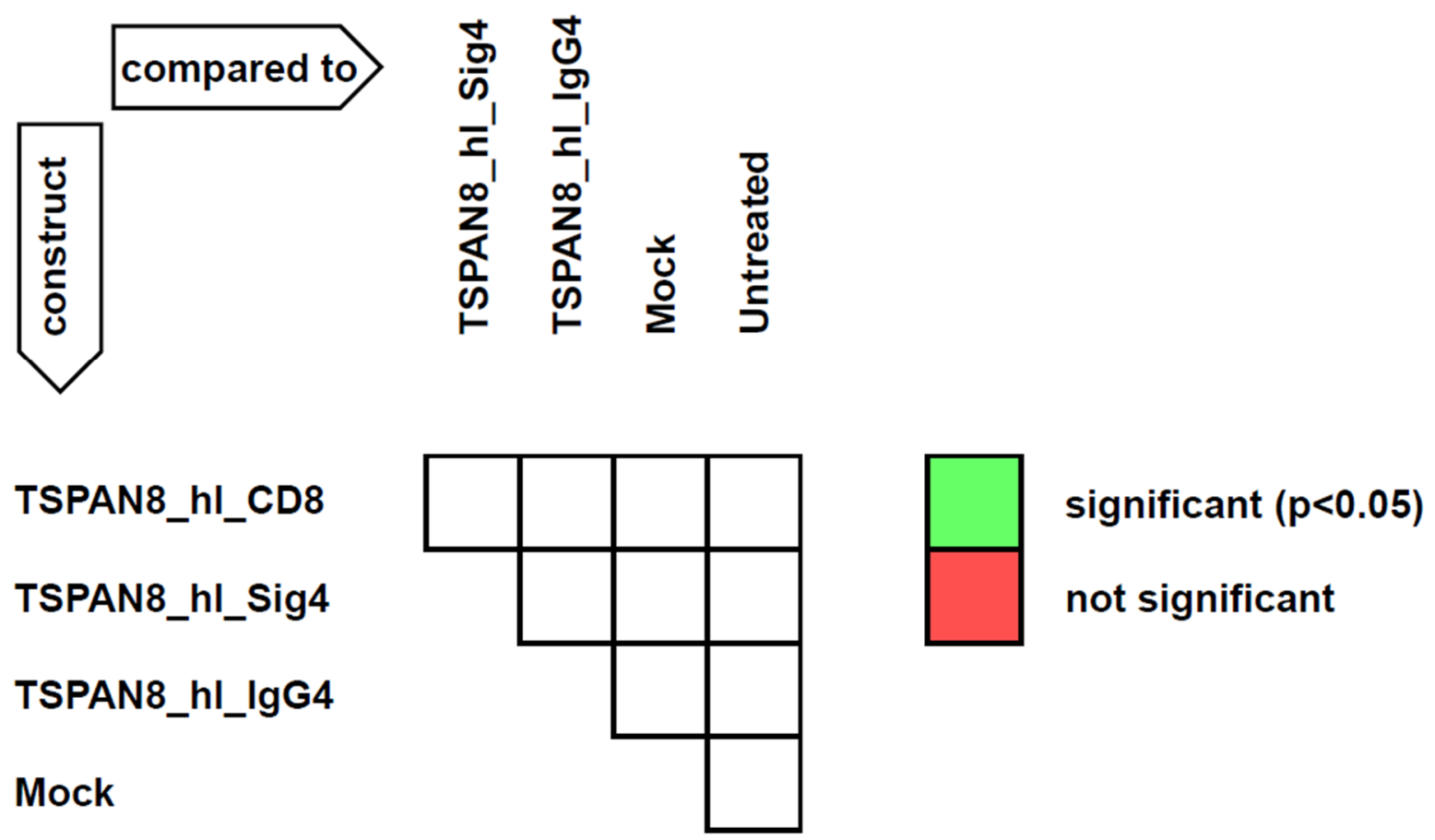

Figure S4: Organization of the pairwise significant matrix for group comparison of in vivo performance of TSPAN8_hl_CD8, TSPAN8_hl_Sig4, TSPAN8_hl_lgG4, Untreated and Mock. PSM $p<0.05$ (green) [one-way ANOVA, multiple comparisons]. 


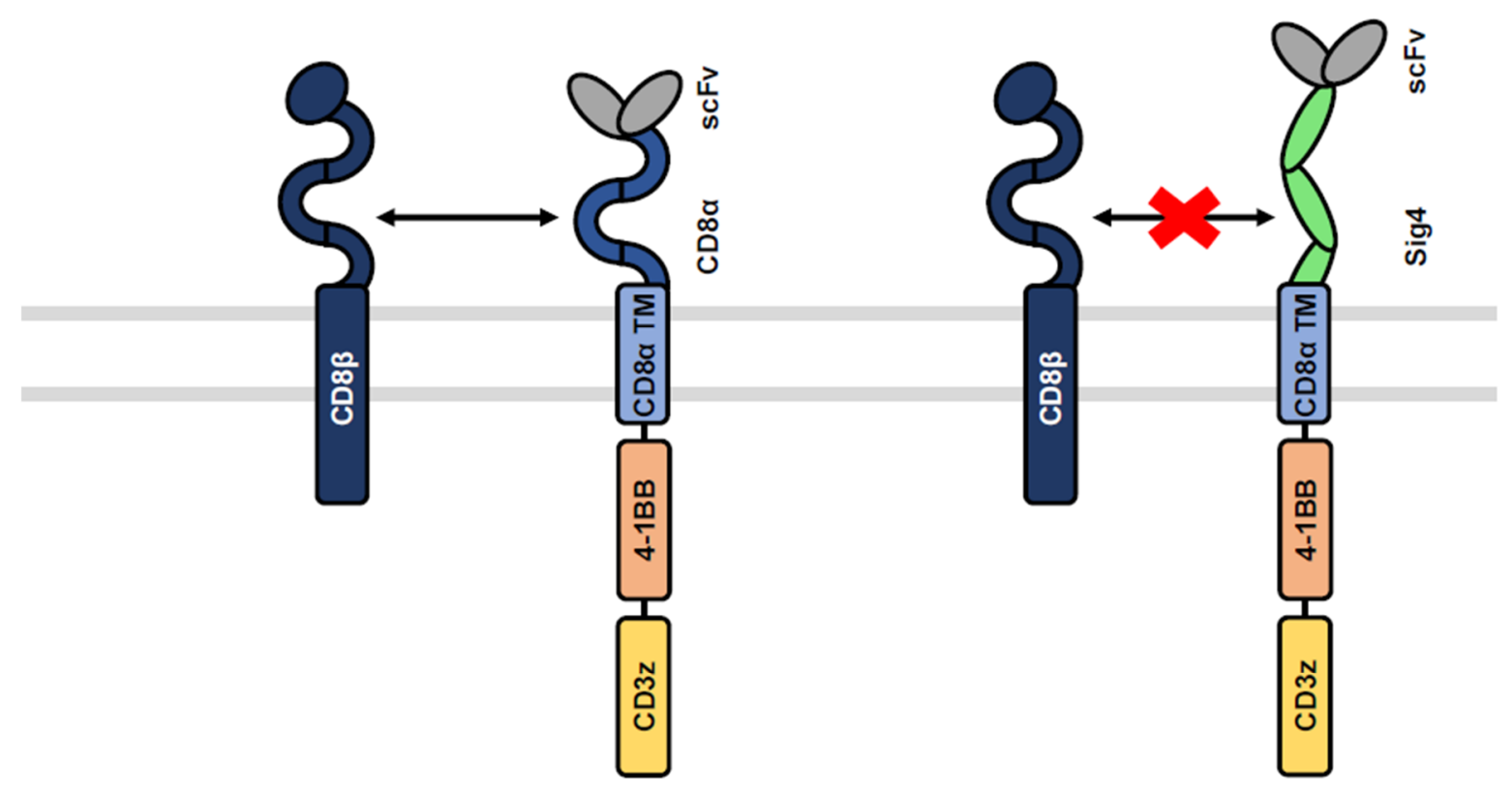

Figure S5: Schematic model of spacer dimerization with natural CD8 $\beta$ leading to increased signaling. 


\section{References}

1. Lee DW, Kochenderfer JN, Stetler-Stevenson M, Cui YK, Delbrook C, Feldman SA, et al. $\mathrm{T}$ cells expressing CD19 chimeric antigen receptors for acute lymphoblastic leukaemia in children and young adults: a phase 1 dose-escalation trial. Lancet. (2015) 385:517-28. doi: 10.1016/S0140-6736(14)61403-3

2. Porter DL, Hwang WT, Frey NV, Lacey SF, Shaw PA, Loren AW, et al. Chimeric antigen receptor $\mathrm{T}$ cells persist and induce sustained remissions in relapsed refractory chronic lymphocytic leukemia. Sci Transl Med. (2015) 7:303ra139. doi: 10.1126/scitranslmed.aac5415

3. Turtle CJ, Hanafi L-A, Berger C, Gooley TA, Cherian S, Hudecek M, et al. CD19 CAR-T cells of defined $\mathrm{CD} 4^{+}: \mathrm{CD}^{+}$composition in adult B cell ALL patients. J Clin Investig. (2016) 126:2123-38. doi: 10.1172/JCI85309

4. Neelapu SS, Locke FL, Bartlett NL, Lekakis LJ, Miklos DB, Jacobson CA, et al. Axicabtagene ciloleucel CAR T-cell therapy in refractory large B-cell lymphoma. $N$ Engl J Med. (2017) 377:2531-44. doi: 10.1056/NEJMoa1707447

5. Schuster SJ, Svoboda J, Chong EA, Nasta SD, Mato AR, Anak Ö, et al. Chimeric antigen receptor t cells in refractory B-cell lymphomas. N Engl J Med. (2017) 377:2545-54. doi: 10.1056/NEJMoa1708566

6. Maude SL, Laetsch TW, Buechner J, Rives S, Boyer M, Bittencourt H, et al. Tisagenlecleucel in children and young adults with B-cell lymphoblastic leukemia. N Engl J Med. (2018) 378:439-48. doi: 10.1056/NEJMoa1709866

7. Park JH, Rivière I, Gonen M, Wang X, Sénéchal B, Curran KJ, et al. Long-term follow-up of CD19 CAR therapy in acute lymphoblastic leukemia. N Engl J Med. (2018) 378:449-59. doi: 10.1056/NEJMoa1709919

8. Gross G, Waks T, Eshhar Z. Expression of immunoglobulin-T-cell receptor chimeric molecules as functional receptors with antibody-type specificity. Proc Natl Acad Sci USA. (1989) 86:10024-8. doi: 10.1073/pnas.86.24.10024

9. Moritz D, Groner B. A spacer region between the single chain antibody- and the CD3 zetachain domain of chimeric $\mathrm{T}$ cell receptor components is required for efficient ligand binding 
10. Darcy PK, Kershaw MH, Trapani JA, Smyth MJ. Expression in cytotoxic T lymphocytes of a single-chain anti-carcinoembryonic antigen antibody. Redirected Fas ligand-mediated lysis of colon carcinoma. Euro J Immunol. (1998) 28:1663-72. doi: 10.1002/sici1521414119980528:051663::aid-immu16633.0.co;2-1

11. Eshhar Z, Waks T, Bendavid A, Schindler DG. Functional expression of chimeric receptor genes in human T cells. J Immunol Methods. (2001) 248:67-76. doi: 10.1016/s0022$17590000343-4$

12. Niederman TMJ, Ghogawala Z, Carter BS, Tompkins HS, Russell MM, Mulligan RC. Antitumor activity of cytotoxic T lymphocytes engineered to target vascular endothelial growth factor receptors. Proc Natl Acad Sci. (2002) 99:7009-14. doi: 10.1073/pnas.092562399

13. Zhang L, Lizzio EF, Chen T, Kozlowski S. Peptide immunization excludes antigen-specific T cells from splenic lymphoid compartments. Euro J Immunol. (2005) 35:776-85. doi: 10.1002/eji.200425479

14. Morgenroth A, Cartellieri M, Schmitz M, Günes S, Weigle B, Bachmann M, et al. Targeting of tumor cells expressing the prostate stem cell antigen. (PSCA) using genetically engineered T-cells. Prostate. (2007) 67:1121-31. doi: 10.1002/pros.20608

15. Zhang H, Snyder KM, Suhoski MM, Maus MV, Kapoor V, June CH, et al. 4-1BB is superior to $\mathrm{CD} 28$ costimulation for generating $\mathrm{CD}^{+}$cytotoxic lymphocytes for adoptive immunotherapy. J Immunol. (2007) 179:4910-8. doi: 10.4049/jimmunol.179.7.4910

16. Barber A, Zhang T, Megli CJ, Wu J, Meehan KR, Sentman CL. Chimeric NKG2D receptorexpressing T cells as an immunotherapy for multiple myeloma. Exp Hematol. (2008) 36:131828. doi: 10.1016/j.exphem.2008.04.010

17. Patel SD, Moskalenko M, Smith D, Maske B, Finer MH, McArthur JG. Impact of chimeric immune receptor extracellular protein domains on T cell function. Gene Therap. (1999) 6:4129. doi: 10.1038/sj.gt.3300831

18. Guest RD, Hawkins RE, Kirillova N, Cheadle EJ, Arnold J, O'Neill A, et al. The role of extracellular spacer regions in the optimal design of chimeric immune receptors: evaluation of four different scfvs and antigens. $J$ Immunother. (2005) 28:203-11. doi: 10.1097/01.cji.0000161397.96582.59 
19. Haso W, Lee DW, Shah NN, Stetler-Stevenson M, Yuan CM, Pastan IH, et al. Anti-CD22chimeric antigen receptors targeting B-cell precursor acute lymphoblastic leukemia. Blood. (2013) 121:1165-74. doi: 10.1182/blood-2012-06-438002

20. Hudecek M, Lupo-Stanghellini M-T, Kosasih PL, Sommermeyer D, Jensen MC, Rader C, et al. Receptor affinity and extracellular domain modifications affect tumor recognition by ROR1-specific chimeric antigen receptor T cells. Clin Cancer Res. (2013) 19:3153-64. doi: 10.1158/1078-0432.CCR-13-0330

21. James SE, Greenberg PD, Jensen MC, Lin Y, Wang J, Till BG, et al. Antigen sensitivity of CD22-specific chimeric TCR is modulated by target epitope distance from the cell membrane. J Immunol. (2008) 180:7028-38. doi: 10.4049/jimmunol.180.10.7028

22. Krenciute G, Krebs S, Torres D, Wu M-F, Liu H, Dotti G, et al. Characterization and functional analysis of scFv-based chimeric antigen receptors to redirect $\mathrm{T}$ cells to IL13R $\alpha 2$ positive glioma. Mol Thera. (2016) 24:354-63. doi: 10.1038/mt.2015.199

23. Hombach A, Hombach AA, Abken H. Adoptive immunotherapy with genetically engineered T cells: modification of the IgG1 Fc 'spacer' domain in the extracellular moiety of chimeric antigen receptors avoids 'off-target' activation and unintended initiation of an innate immune response. Gene Thera. (2010) 17:1206-13. doi: 10.1038/gt.2010.91

24. Almåsbak H, Walseng E, Kristian A, Myhre MR, Suso EM, Munthe LA, et al. Inclusion of an IgG1-Fc spacer abrogates efficacy of CD19 CAR T cells in a xenograft mouse model. Gene Thera. (2015) 22:391-403. doi: 10.1038/gt.2015.4

25. Hudecek M, Sommermeyer D, Kosasih PL, Silva-Benedict A, Liu L, Rader C, et al. The nonsignaling extracellular spacer domain of chimeric antigen receptors is decisive for in vivo antitumor activity. Cancer Immunol Res. (2015) 3:125-35. doi: 10.1158/2326-6066.CIR-140127

26. Watanabe N, Bajgain P, Sukumaran S, Ansari S, Heslop HE, Rooney CM, et al. Fine-tuning the CAR spacer improves T-cell potency. Oncoimmunology. (2016) 5:e1253656. doi: $10.1080 / 2162402 X .2016 .1253656$

27. Jonnalagadda M, Mardiros A, Urak R, Wang X, Hoffman LJ, Bernanke A, et al. Chimeric antigen receptors with mutated $\mathrm{IgG} 4 \mathrm{Fc}$ spacer avoid fc receptor binding and improve $\mathrm{T}$ cell persistence and antitumor efficacy. Mol Thera. (2015) 23:757-68. doi: 10.1038/mt.2014.208 
28. Jensen MC, Popplewell L, Cooper LJ, DiGiusto D, Kalos M, Ostberg JR, et al. Antitransgene rejection responses contribute to attenuated persistence of adoptively transferred CD20/CD19-specific chimeric antigen receptor redirected T cells in humans. Biol Blood Marrow Transplant. (2010) 16:1245-56. doi: 10.1016/j.bbmt.2010.03.014

29. Savoldo B, Ramos CA, Liu E, Mims MP, Keating MJ, Carrum G, et al. CD28 costimulation improves expansion and persistence of chimeric antigen receptor-modified $\mathrm{T}$ cells in lymphoma patients. J Clin Investig. (2011) 121:1822-6. doi: 10.1172/JCI46110

30. Till BG, Jensen MC, Wang J, Qian X, Gopal AK, Maloney DG, et al. CD20-specific adoptive immunotherapy for lymphoma using a chimeric antigen receptor with both CD28 and 4-1BB domains: pilot clinical trial results. Blood. (2012) 119:3940-50. doi: 10.1182/blood2011-10-387969

31. Gargett T, Yu W, Dotti G, Yvon ES, Christo SN, Hayball JD, et al. GD2-specific CAR T cells undergo potent activation and deletion following antigen encounter but can be protected from activation-induced cell death by PD-1 blockade. Mol Therap. (2016) 24:1135-49. doi: $10.1038 / \mathrm{mt} .2016 .63$

32. Brown CE, Alizadeh D, Starr R, Weng L, Wagner JR, Naranjo A, et al. Regression of glioblastoma after chimeric antigen receptor T-cell therapy. N Engl J Med. (2016) 375:25619. doi: 10.1056/NEJMoa1610497

33. Wang X, Popplewell LL, Wagner JR, Naranjo A, Blanchard MS, Mott MR, et al. Phase 1 studies of central memory-derived CD19 CAR T-cell therapy following autologous HSCT in patients with B-cell NHL. Blood. (2016) 127:2980-90. doi: 10.1182/blood-2015-12-686725

34. Abramson JS, Palomba ML, Gordon LI, Lunning MA, Wang ML, Arnason JE, et al. Pivotal safety and efficacy results from transcend NHL 001, a multicenter phase 1 study of lisocabtagene maraleucel (liso-cel) in relapsed/refractory (R/R) large B cell lymphomas. Blood. (2019) 134(Suppl.1):241. doi: 10.1182/blood-2019-127508

35. Crocker PR, Varki A. Siglecs, sialic acids and innate immunity. Trends Immunol. (2001) 22:337-42. doi: 10.1016/s1471-4906(01)01930-5

36. Crocker PR, Paulson JC, Varki A. Siglecs and their roles in the immune system. Nat Rev Immunol. (2007) 7:255-66. doi: 10.1038/nri2056 
37. Jensen M, Tan G, Forman S, Wu AM, Raubitschek A. CD20 is a molecular target for scFvFc:zeta receptor redirected T cells: implications for cellular immunotherapy of CD20 ${ }^{+}$ malignancy. Biol Blood Marrow Transplant. (1998) 4:75-83. doi: 10.1053/bbmt.1998.v4.pm9763110

38. Apel M, Brüning M, Granzin M, Essl M, Stuth J, Blaschke J, et al. Integrated clinical scale manufacturing system for cellular products derived by magnetic cell separation, centrifugation and cell culture. Chem Ingen Tech. (2013) 85:103-10. doi: 10.1002/cite.201200175

39. Lock D, Mockel-Tenbrinck N, Drechsel K, Barth C, Mauer D, Schaser T, et al. Automated manufacturing of potent CD20-directed chimeric antigen receptor t cells for clinical use. Hum Gene Ther. (2017) 28:914-25. doi: 10.1089/hum.2017.111

40. Al Rawashdeh W, Zuo S, Melle A, Appold L, Koletnik S, Tsvetkova Y, et al. Noninvasive assessment of elimination and retention using CT-FMT and kinetic whole-body modeling. Theranostics. (2017) 7:1499-510. doi: 10.7150/thno.17263

41. Till BG, Jensen MC, Wang J, Chen EY, Wood BL, Greisman HA, et al. Adoptive immunotherapy for indolent non-hodgkin lymphoma and mantle cell lymphoma using genetically modified autologous CD20-specific T cells. Blood. (2008) 112:2261-71. doi: 10.1182/blood-2007-12-128843

42. Polyak MJ, Deans JP. Alanine-170 and proline-172 are critical determinants for extracellular CD20 epitopes; heterogeneity in the fine specificity of CD20 monoclonal antibodies is defined by additional requirements imposed by both amino acid sequence and quaternary structure. Blood. (2002) 99:3256-62. doi: 10.1182/blood.v99.9.3256

43. Macauley MS, Crocker PR, Paulson JC. Siglec-mediated regulation of immune cell function in disease. Nat Rev Immunol. (2014) 14:653-66. doi: 10.1038/nri3737

44. Bornhöfft KF, Goldammer T, Rebl A, Galuska SP. Siglecs: a journey through the evolution of sialic acid-binding immunoglobulin-type lectins. Dev Comparat Immunol. (2018) 86:21931. doi: 10.1016/j.dci.2018.05.008

45. Srivastava S, Riddell SR. Engineering CAR-T cells: design concepts. Trends Immunol. (2015) 36:494-502. doi: 10.1016/j.it.2015.06.004

46. Riddell SR, Elliott M, Lewinsohn DA, Gilbert MJ, Wilson L, Manley SA, et al. T-cell mediated rejection of gene-modified HIV-specific cytotoxic T lymphocytes in HIV-infected patients. Nat Med. (1996) 2:216-23. doi: 10.1038/nm0296-216 
47. Berger C, Flowers ME, Warren EH, Riddell SR. Analysis of transgene-specific immune responses that limit the in vivo persistence of adoptively transferred HSV-TK-modified donor T cells after allogeneic hematopoietic cell transplantation. Blood. (2006) 107:2294-302. doi: 10.1182/blood-2005-08-3503

48. Hernández-Caselles T, Martínez-Esparza M, Pérez-Oliva AB, Quintanilla-Cecconi AM, García-Alonso A, Alvarez-López DMR, et al. A study of CD33. (SIGLEC-3) antigen expression and function on activated human T and NK cells: two isoforms of CD33 are generated by alternative splicing. J Leukocyte Biol. (2006) 79:46-58. doi: 10.1189/jlb.0205096 49. Laszlo GS, Harrington KH, Gudgeon CJ, Beddoe ME, Fitzgibbon MP, Ries RE, et al. Expression and functional characterization of CD33 transcript variants in human acute myeloid leukemia. Oncotarget. (2016) 7:43281-94. doi: 10.18632/oncotarget.9674

50. Pérez-Oliva AB, Martínez-Esparza M, Vicente-Fernández JJ, Corral-San Miguel R, GarcíaPeñarrubia P, Hernández-Caselles T. Epitope mapping, expression and post-translational modifications of two isoforms of CD33 (CD33M and CD33m) on lymphoid and myeloid human cells. Glycobiology. (2011) 21:757-70. doi: 10.1093/glycob/cwq220

51. Künkele A, Johnson AJ, Rolczynski LS, Chang CA, Hoglund V, Kelly-Spratt KS, et al. Functional tuning of CARs reveals signaling threshold above which $\mathrm{CD} 8^{+} \mathrm{CTL}$ antitumor potency is attenuated due to cell Fas-FasL-dependent AICD. Cancer Immunol Res. (2015) 3:368-79. doi: 10.1158/2326-6066.CIR-14-0200

52. Valitutti S, Müller S, Dessing M, Lanzavecchia A. Signal extinction and $\mathrm{T}$ cell repolarization in T helper cell-antigen-presenting cell conjugates. Euro J Immunol. (1996) 26:2012-6. doi: 10.1002/eji.1830260907

53. Itoh Y, Germain RN. Single cell analysis reveals regulated hierarchical $\mathrm{T}$ cell antigen receptor signaling thresholds and intraclonal heterogeneity for individual cytokine responses of CD4 ${ }^{+}$T cells. J Exp Med. (1997) 186:757-66. doi: 10.1084/jem.186.5.757

54. Hemmer B, Vergelli M, Gran B, Ling N, Conlon P, Pinilla C, et al. Predictable TCR antigen recognition based on peptide scans leads to the identification of agonist ligands with no sequence homology. J Immunol. (1998) 160:3631-6

55. Auphan-Anezin N, Verdeil G, Schmitt-Verhulst A-M. Distinct thresholds for CD8 T cell activation lead to functional heterogeneity: CD8 T cell priming can occur independently of cell division. J Immunol. (2003) 170:2442-8. doi: 10.4049/jimmunol.170.5.2442 
56. Faroudi M, Zaru R, Paulet P, Müller S, Valitutti S. Cutting edge: T lymphocyte activation by repeated immunological synapse formation and intermittent signaling. J Immunol. (2003) 171:1128-32. doi: 10.4049/jimmunol.171.3.1128

57. Liu Z, Gerner MY, Van Panhuys N, Levine AG, Rudensky AY, Germain RN. Immune homeostasis enforced by co-localized effector and regulatory T cells. Nature. (2015) 528:22530. doi: 10.1038/nature16169

58. Caruso HG, Hurton LV, Najjar A, Rushworth D, Ang S, Olivares S, et al. Tuning sensitivity of CAR to EGFR density limits recognition of normal tissue while maintaining potent antitumor activity. Cancer Res. (2015) 75:3505-18. doi: 10.1158/0008-5472.can-15-0139

59. Ghorashian S, Kramer AM, Onuoha S, Wright G, Bartram J, Richardson R, et al. Enhanced CAR T cell expansion and prolonged persistence in pediatric patients with ALL treated with a low-affinity CD19 CAR. Nat Med. (2019) 25:1408-14. doi: 10.1038/s41591-019-0549-5

60. Leahy DJ, Axel R, Hendrickson WA. Crystal structure of a soluble form of the human T cell coreceptor CD8 at 2.6 a resolution. Cell. (1992) 68:1145-62. doi: 10.1016/00928674(92)90085-q

61. Pang S, Zhang L, Wang H, Yi Z, Li L, Gao L, et al. CD8(+) T cells specific for beta cells encounter their cognate antigens in the islets of NOD mice. Euro J Immunol. (2009) 39:271624. doi: 10.1002/eji.200939408

62. Bosselut R, Kubo S, Guinter T, Kopacz JL, Altman JD, Feigenbaum L, et al. Role of CD8 $\beta$ domains in CD8 coreceptor function: importance for MHC I binding, signaling, and positive selection of CD8 ${ }^{+} \mathrm{T}$ cells in the thymus. Immunity. (2000) 12:409-18. doi: 10.1016/S10747613(00)80193-4

63. Brudno JN, Kochenderfer JN. Toxicities of chimeric antigen receptor T cells: recognition and management. Blood. (2016) 127:3321-30. doi: 10.1182/blood-2016-04-703751

64. Brentjens RJ, Davila ML, Riviere I, Park J, Wang X, Cowell LG, et al. CD19-targeted T cells rapidly induce molecular remissions in adults with chemotherapy-refractory acute lymphoblastic leukemia. Sci Transl Med. (2013) 5:177ra138. doi: 10.1126/scitranslmed.3005930

65. Grupp SA, Kalos M, Barrett D, Aplenc R, Porter DL, Rheingold SR, et al. Chimeric antigen receptor-modified T cells for acute lymphoid leukemia. N Engl J Med. (2013) 368:1509-18. doi: 10.1056/NEJMoa1215134 
66. Maude SL, Barrett D, Teachey DT, Grupp SA. Managing cytokine release syndrome associated with novel $T$ cell-engaging therapies. Cancer J. (2014) 20:119-22. doi: 10.1097/PPO.0000000000000035

67. Kochenderfer JN, Dudley ME, Kassim SH, Somerville RP, Carpenter RO, StetlerStevenson M, et al. Chemotherapy-refractory diffuse large B-cell lymphoma and indolent Bcell malignancies can be effectively treated with autologous T cells expressing an anti-CD19 chimeric antigen receptor. J Clin Oncol. (2015) 33:540-9. doi: 10.1200/jco.2014.56.2025

68. Hay KA, Hanafi L-A, Li D, Gust J, Liles WC, Wurfel MM, et al. Kinetics and biomarkers of severe cytokine release syndrome after CD19 chimeric antigen receptor-modified T-cell therapy. Blood. (2017) 130:2295-306. doi: 10.1182/blood-2017-06-793141

69. Bartsch U, Kirchhoff F, Schachner M. Immunohistological localization of the adhesion molecules L1, N-CAM, and MAG in the developing and adult optic nerve of mice. J Comparat Neurol. (1989) 284:451-62. doi: 10.1002/cne.902840310

70. Trapp BD, Andrews SB, Cootauco C, Quarles R. The myelin-associated glycoprotein is enriched in multivesicular bodies and periaxonal membranes of actively myelinating oligodendrocytes. J Cell Biol. (1989) 109:2417-26. doi: 10.1083/jcb.109.5.2417

71. McKerracher L, David S, Jackson DL, Kottis V, Dunn RJ, Braun PE. Identification of myelin-associated glycoprotein as a major myelin-derived inhibitor of neurite growth. Neuron. (1994) 13:805-11. doi: 10.1016/0896-6273(94)90247-x

72. Mukhopadhyay G, Doherty P, Walsh FS, Crocker PR, Filbin MT. A novel role for myelinassociated glycoprotein as an inhibitor of axonal regeneration. Neuron. (1994) 13:757-67. doi: 10.1016/0896-62739490042-6

73. Schachner M, Bartsch U. Multiple functions of the myelin-associated glycoprotein MAG (siglec-4a) in formation and maintenance of myelin. Glia. (2000) 29:154-65. doi: 10.1002/sici1098-11362000011529:2154::aid-glia93.0.co;2-3

74. Collins BE, Ito H, Sawada N, Ishida H, Kiso M, Schnaar RL. Enhanced binding of the neural siglecs, myelin-associated glycoprotein and Schwann cell myelin protein, to Chol-1 (alpha-series) gangliosides and novel sulfated Chol-1 analogs. J Biol Chem. (1999) 274:3763743. doi: $10.1074 /$ jbc.274.53.37637 
75. Tang S, Shen YJ, DeBellard ME, Mukhopadhyay G, Salzer JL, Crocker PR, et al. Myelinassociated glycoprotein interacts with neurons via a sialic acid binding site at ARG118 and a distinct neurite inhibition site. J Cell Biol. (1997) 138:1355-66. doi: 10.1083/jcb.138.6.1355

76. May AP, Robinson RC, Vinson M, Crocker PR, Jones EY. Crystal structure of the Nterminal domain of sialoadhesin in complex with 3 ' sialyllactose at 1.85 A resolution. Mol Cell. (1998) 1:719-28. doi: 10.1016/s1097-2765(00)80071-4

77. Zaccai NR, Maenaka K, Maenaka T, Crocker PR, Brossmer R, Kelm S, et al. Structureguided design of sialic acid-based siglec inhibitors and crystallographic analysis in complex with sialoadhesin. Structure. (2003) 11:557-67. doi: 10.1016/s0969-2126(03)00073-x

78. Lehmann F, Gäthje H, Kelm S, Dietz F. Evolution of sialic acid-binding proteins: molecular cloning and expression of fish siglec-4. Glycobiology. (2004) 14:959-68. doi: 10.1093/glycob/cwh120

79. Domeniconi M, Cao Z, Spencer T, Sivasankaran R, Wang K, Nikulina E, et al. Myelinassociated glycoprotein interacts with the Nogo66 receptor to inhibit neurite outgrowth. Neuron. (2002) 35:283-90. doi: 10.1016/s0896-6273(02)00770-5

80. Liu BP, Fournier A, GrandPré T, Strittmatter SM. Myelin-associated glycoprotein as a functional ligand for the Nogo-66 receptor. Science. (2002) 297:1190-3. doi: 10.1126/science. 1073031

81. Venkatesh K, Chivatakarn O, Lee H, Joshi PS, Kantor DB, Newman BA, et al. The Nogo66 receptor homolog $\mathrm{NgR} 2$ is a sialic acid-dependent receptor selective for myelin-associated glycoprotein. J Neurosci. (2005) 25:808-22. doi: 10.1523/jneurosci.4464-04.2005

82. Atwal JK, Pinkston-Gosse J, Syken J, Stawicki S, Wu Y, Shatz C, et al. PirB is a functional receptor for myelin inhibitors of axonal regeneration. Science. (2008) 322:967. doi: $10.1126 /$ science. 1161151

83. Robak LA, Venkatesh K, Lee H, Raiker SJ, Duan Y, Lee-Osbourne J, et al. Molecular basis of the interactions of the Nogo-66 receptor and its homolog NgR2 with myelin-associated glycoprotein: development of NgROMNI-Fc, a novel antagonist of CNS myelin inhibition. $J$ Neurosci. (2009) 29:5768-83. doi: 10.1523/JNEUROSCI.4935-08.2009

84. Cao Z, Qiu J, Domeniconi M, Hou J, Bryson JB, Mellado W, et al. The inhibition site on myelin-associated glycoprotein is within Ig-domain 5 and is distinct from the sialic acid binding site. J Neurosci. (2007) 27:9146-54. doi: 10.1523/jneurosci.2404-07.2007 
85. Arquint M, Roder J, Chia LS, Down J, Wilkinson D, Bayley H, et al. Molecular cloning and primary structure of myelin-associated glycoprotein. Proc Natl Acad Sci USA. (1987) 84:600-4. doi: 10.1073/pnas.84.2.600

86. Lai C, Brow MA, Nave KA, Noronha AB, Quarles RH, Bloom FE, et al. Two forms of 1B236/myelin-associated glycoprotein, a cell adhesion molecule for postnatal neural development, are produced by alternative splicing. Proc Natl Acad Sci USA. (1987) 84:433741. doi: $10.1073 /$ pnas.84.12.4337 


\section{Discussion}

\subsection{Target discovery}

CAR T cells do not only harbor great potential for treatment of cancer patients but also represent a substantial challenge for physicians and researchers. These highly sensitive cells are able to kill almost any cell in the human body to which they are specific for and can persist for several months or even years, hence delivering a long-time immunity (Ghorashian et al., 2019). This is in stark contrast to small molecule agents or chemotherapies, which in general have half-lives of days or hours (Lennernäs et al., 2003). While this extraordinary efficacy and persistence is a strong advantage, it can become problematic when directed towards non-tumor cells, making the choice of the target antigen of superior importance. Knowing the insufficiencies of established PDAC CAR targets, the first objective of this study aimed at identifying novel target candidates followed by a characterization of their putative safety profile by analyzing somatic tissue expression.

\subsubsection{On-tumor specificity}

Our starting point for this study was the flow cytometric screening of 371 surface antigens on a heterogenous set of dissociated tumor samples from human PDAC PDX mouse models with 15 different mutational backgrounds. Most of the surface antigens identified on cells of the xenografts were differentially expressed, mirroring the diversity of PDAC. From this group three surface antigens displayed a homogeneous high expression: CD66c, which belongs to the protein family of carcinoembryonic antigens (Hammarström, 1999); the tetraspanin protein family member TSPAN8 (Charrin et al., 2014) and the glycosylation CLA, which is a specialized variant of the surface protein SELPLG (Fuhlbrigge et al., 1997; Ohmori et al., 2006). The strong expression of these target candidates on PDXs could be corroborated later on primary human PDACs. These targets qualify thus for an application on a broader range of patients. This may not necessarily be true for CD318, another surface protein also known as CDCP1, which was expressed on all of the human tumors investigated by us, but not on all cells categorized as tumor cells using flow cytometry and EpCAM as tumor marker. Nonetheless, targeting only a subpopulation of a tumor may be enough to elicit cytokine release strong enough for an intrinsic immunogenic response (Nemunaitis, 2005). Furthermore, it has to be considered that EpCAM is a general marker for the epithelial linage (Keller et al., 2019). Hence, not every $\mathrm{EpCAM}^{+}$cell is a tumor cell. This combined with an overall lower expression of CD318 as compared to the other three targets may lead to the underestimation of its expression in this flow based assay. Gating strategy is of critical importance, especially when low 
expressed markers are investigated (Verschoor et al., 2015). Thus, we verified CD318 expression on all tumor cells on two additional human PDACs using microscopy as a second method. Optical methods have the advantage that structural information of a cell can be used to identify tumor cells and are also used in clinical diagnosis (Kleeff et al., 2016).

We chose this multifaceted set of PDXs in terms of mutational diversity intentionally, as PDAC itself has a very heterogenous mutational landscape (Waddell et al., 2015; Witkiewicz et al., 2015; Bailey et al., 2016). Most of current PDAC CAR targets are only specific for subpopulations of patients (Akce et al., 2018), which may be a consequence of the high plasticity of PDAC. Hence, we included these diverse PDAC mouse models in order to identify targets expressed on the majority of tumors, which is indeed the case for our four targets CLA, CD66c, CD318 and TSPAN8.

Both, the diversity of target models investigated as well as the sheer amount of target candidates measured are unique to this study and unprecedented in the context of CAR T cells. Another study using a similar experimental design was conducted by Mount and colleagues. However, they applied a panel containing only 242 antibodies, on four different patient derived cell lines of diffuse intrinsic pontine glioma (Mount et al., 2018). They identified GD2 as most promising target but did not perform a subsequent safety analysis, which is in contrast to our study. We included a thorough safety assessment and found our targets to have safer expression profiles as previous CAR targets as discussed in the next chapter. Other studies comparing a broad set of surface antigen expression on a diversity of tumor cells with antibody based methods are missing.

We chose a predefined set of antibodies as investigation basis, as it comes with several benefits for subsequent target identification and CAR development. The antigen expression can be measured and quantified directly on the protein level, which is in contrast to RNA sequencing and proteomics based methods. Also, the subsequent specificity of the CAR T cells remains the same as observed in the binding studies, as the CAR binder and the antibody used for discovery have the same paratope. These traits simplify subsequent CAR analysis. The fact that the design of functional CARs is a rather elaborate process with its own hurdles is exemplified by several other studies, which suggested novel CAR target candidates but did not proceed to CAR cloning and testing (Orentas et al., 2012; Bosse et al., 2017; Perna et al., 2017). Another inherent problem of target discovery using omics based methods is their predictive nature. Algorithms have to be applied on the collected data in order to identify transcripts meant for later surface expression. Transmembrane domains are highly complex and variable in structure, which make 
them difficult to define (Koehler Leman et al., 2015; Sezgin et al., 2017). Using transcriptomics has gained huge interest as its costs declined and it became easier to use (Timp and Timp, 2020). RNA sequencing however is only highlighting the first step from the gene to its final product. Until mRNA is translated and a protein reaches the cell surface, a lot of interferences are to be expected. Post-translational modifications may take place and the cellular transportation system is highly complex, leading to not necessarily correlating mRNA and cell surface protein levels (Schwanhäusser et al., 2011; Jiang et al., 2020). In addition, not every algorithm includes target identification of the several molecule classes, which can be found on a cell surface. Phospholipids, carbohydrates and proteins can all be detected simultaneously using antibodies, while for mass spectrometry detection of the several classes the experimental set-up needs to be changed (Coman et al., 2016). All these difficulties lead to increasing the chance to include false positives. Perna et al. performed proteomic and transcriptomic studies and narrowed down the field of candidates for the treatment of AML from 4942 to 24 (Perna et al., 2017). Of these 24 candidates, the expression of only 9 could be verified on AML patient cells using antibodybased flow cytometry. This exemplifies that target discovery based on transcriptomic and proteomic approaches can lead to valid novel target candidates, but it is crucial to further experimentally validate those, while this is already included in antibody-based screening methods, as applied in this study. We did not rely on predictive omic workflows and could demonstrate the presence of our targets using flow cytometry on PDXs and dissociated human tumors, as well as microscopy on human PDACs tissue samples.

A clear disadvantage of using pre-defined antibody arrays for target discovery is the restriction of possible targets to the number and specificities of antibodies contained in the array. While our target discovery panel contained almost 400 antibodies, modern mass spectrometry methods are able to identify 3.000 surface proteins (Bausch-Fluck et al., 2018; Jiang et al., 2020). Our approach had thus a 7.5 times smaller field of potential targets. Moreover, these antibodies haven been defined and validated on epitopes already known. This is why we were not able to unravel truly novel surface antigens, as CLA, CD66c, CD318 and TSPAN8 are already known in other contexts (Fuhlbrigge et al., 1997; Hammarström, 1999; Wortmann et al., 2009; Charrin et al., 2014). Using an antibody array nullifies chances of discovering a truly novel target, in contrast to omics based approaches.

\subsubsection{Off-tumor safety profiling}

Although maybe the most critical part of CAR T cell therapy, the off-tumor safety evaluation of potential target candidates has often been conducted less thoroughly than necessary. Given 
the known shortcomings and sometimes contradictory predictions of transcriptomic and proteomic data, we aimed at combining the information of several databases. In difference to previous studies (Bosse et al., 2017; Perna et al., 2017), we tried not to bioinformatically predict the absence of CAR target candidates but rather ordered them with respect to the likelihood of overall lowest expression. This helped narrowing down the field of target candidates from 50 to 8 , which had to be analyzed by antibody based methods, for the reasons described above, to verify absence of the target candidate.

Of these 8 target candidates being specific to tumor cells as shown by flow cytometry and cyclic IF, our off-tumor expression ranking indicated that CLA, CD66c, CD318 and TSPAN8 had the most restricted expression profiles on healthy tissues. Interestingly, they ranked safer, than most of the previously used CAR targets, like HER2 and EGFR. Unsurprisingly, for all four target candidates, some restricted off-tumor expression was predicted. In the case of CLA, this prediction has to be analyzed carefully. CLA is a glycosylation and the ranking was performed using the suspected backbone protein SELPLG (Fuhlbrigge et al., 1997). This might not reflect the real expression pattern, which is supposedly more restricted as CLA is only present on a subset of SELPLG expressing cells.

Subsequently, we first aimed to generate functional CAR constructs specific for these four candidates, as analysis for clinical applicability is irrelevant, when no functional CAR constructs are available. This strategy succeeded, as no functional CLA CARs could be generated due to CAR T cell self-recognition, making a continued safety analysis obsolete. We screened the remaining three candidates on 17 tissues that have been identified by our bioinformatical safety assessment as problematic, such as liver, colon, lung and kidney, using cyclic IF and additionally on whole blood using flow cytometry. While CD318 was not detectable on almost all tissues, CD66c and TSPAN8 displayed some scattered expression, especially in the gastro-intestinal tract. Therefore, further studies should address strategies to increase CAR T cell safety as discussed below. This verification step is of utmost importance and was not always conducted for some established CAR targets (Katari et al., 2011; Maliar et al., 2012; Abate-Daga et al., 2014; Posey et al., 2016), which also has historical reasons.

Around the millennium change CAR T cell therapy was still infantile and comprehensive expression databases were not existent yet (Barrett et al., 2012; Kolesnikov et al., 2014). Machines and computers to generate and analyze the huge amount of data that they can do today were not available. What was available, were toxicity data from clinically approved antibodies. Some of the CAR targeting antigens that are used in current PDAC therapy have been targeted 
by antibody therapies before, such as HER2 and EGFR (Hudis, 2007; Martinelli et al., 2009; Costa and Czerniecki, 2020). These therapies proofed to be safe. However, CAR T cells as "a living drug" have different and hard to control pharmacokinetics and are more powerful than antibodies (Szöör et al., 2020). While low off-tumor expression may be tolerable for antibody based therapies due to controllable pharmacokinetics allowing for a therapeutic window, this can lead to serious adverse events in case of CAR T cell administration, as exemplified by therapies directed to HER2. While the antibody trastuzumab has been in clinical use for years, HER2 specific CAR T cell administration lead to a deadly adverse event (Hudis, 2007; Morgan et al., 2010). Of note, our off-tumor expression evaluation ranked these targets lower as the ones suggested by this study. A meticulous investigation of expression patterns of HER2, as well as a holistic CAR functionality testing as conducted for the novel targets CLA, CD318, CD66c and TSPAN8 from this study, would have helped to gather a better informed understanding of the expression pattern of the target and CAR T cell behavior towards it. As response to potential off-tumor toxicities, alternative injection routes (Katz et al., 2015) or finetuned CARs (Caruso et al., 2015) could have been applied.

When using refined clinical protocols, administration of HER2 specific CAR T cells can be save, without severe side effects (Ahmed et al., 2015; Hegde et al., 2017). Apparently, a fine interplay between CAR $\mathrm{T}$ cell dose, CAR expression and target expression determines a therapeutic window that can be exploited (Watanabe et al., 2018). Following this suggestion, newer studies implemented methods to determine target overexpression in tumor tissue as compared to normal tissue (Orentas et al., 2012; Bosse et al., 2017; Perna et al., 2017). Bosse and colleagues defined a log-fold change of RNA expression of above one as sufficient, Orentas et al. calculated a $\mathrm{T}$ value based on RNA expression that had to be higher than 10 for differential expression between diseased and healthy tissue, while Perna et al. categorized expression differences in low, medium and high depending on gaussian distribution and distance to the standard deviation for mass spectrometry data and transcriptomic databases. While these methods may be using arbitrarily chosen thresholds, they are precise in giving relative expression differences, which can be used for narrowing down the field of target candidates. However, when basal tissue expression is strong already and tumor expression even stronger, the difference still has a big margin, but CAR T cells would most likely act on healthy tissues, as basal expression is already above the activation threshold. If these differences will be able to open up a therapeutic window sufficient for treatment remains unclear. Most important, each database has an intrinsic detection limit. This is unproblematic for on-tumor detection, as investigators are looking for highly expressed surface molecules. For off-tumor expression 
evaluation however, it is not clear, if the detection limit of the respective experimental method matches the detection threshold of CAR T cells. Thus, also the final step in off-tumor expression evaluation should be antibody based binding investigations as conducted in this study. This is exemplified by the results of Bosse et al, who found mRNA expression of their target GPC2 for neuroblastoma only slightly above background in skin and testis, while their IHC analysis on 36 healthy human tissues revealed expression on $25 \%$ of the tissues ( 6 weak cytoplasmic, 1 strong cytoplasmic, 2 weak membrane bound)(Bosse et al., 2017). Also Perna et al. identified 9 target candidates using transcriptomic and proteomic methods (Perna et al., 2017). Notably, they did not include spleen and blood in their analysis and consequently had to eliminate 5 more candidates after flow cytometry based measurements revealed their expression on T cells.

Being aware of this predicament, we decided to first circumvent absolute statements about tissue expression. Instead, we prioritized our tumor specific candidates by using several databases that derived their data from different experimental methods. Human Protein Atlas (Uhlén et al., 2015), GTEx (https://gtexportal.org/), Human Proteome Map (Kim et al., 2014) and ProteomicsDB (Wilhelm et al., 2014) offer data from RNA sequencing, proteomics by mass spectrometry and antibody based methods, all of them with different advantages and limitations as described above. This led finally to an informed understanding of the expression patterns of our novel targets in healthy tissues. That this is a valid approach was further underlined, as our novel target candidates were higher ranked as targets already under investigation, such as CD133, EGFR or HER2, indicating a higher healthy tissue expression for previously known targets.

\subsubsection{Adding crucial information by spatial analysis of protein expression on the single cell level}

This study together with previous work from others pointed out the importance of antibody based validation of predicted target candidates from transcriptomics, proteomics or bioinformatical databases (Bosse et al., 2017; Perna et al., 2017; Reinhard et al., 2020). Our newly developed cyclic IF device proved to be especially valuable in that regard. While traditional IF microscopy is limited to simultaneous staining of up to six conjugates (Lin et al., 2015), we performed cyclic IF runs, which marked 98 and 107 antigens on the very same tissue section, leading to direct verification if an antigen is expressed on the cell surface or not. This enabled us to compare a broad range of potential target candidates and analyze their tissue specificity and localize their expression to specific cells within the tumors. We confirmed, that our target candidates CLA, CD66c, CD318 and TSPAN8 are highly specific to PDAC tumor 
cells. This was different for target candidates like CD51, CD59 or CD195. They proved to be expressed in PDAC tissue samples; however, not on the tumor cells themselves but rather in the desmoplastic stroma, disqualifying them for CAR T cell therapy. Future studies may take advantage of this multiplexed analysis, as it was conducted in this study.

Defining the localization of certain target candidates is of special importance in the context of off-tumor expression and CAR safety. The bioinformatical databases used in this study do not resolve expression profiles to a single cell level, but rather display the average over all cells of an organ. Thus, target candidates may be expressed on indispensable organs, but within these organs might be restricted to non-vital cell types. This information is lost during bulk analysis. For example, our bioinformatical analysis predicted some expression of CD66c in the liver, while we could show, that this is a marginal amount of blood cells. Expression was also predicted within skin tissue. We could clarify, that this expression is restricted to hair follicle cells, which are non-essential. Although a general trend can be observed to conduct research more often on single cell levels (Angerer et al., 2017; Marx, 2019), this was not the case for the databases used at the time of this study. Analysis of tissues by cyclic IF can fill this gap as shown by our results.

Not only does cyclic IF help to delineate expression on single cell level, but also the polarization of a cell can be captured. Here, we demonstrated expression of CD66c in colon epithelium. However, CD66c is localized to the luminal part of the colon, making it unlikely to be presented to immune cells. Thus, although expressed in colon, CD66c could still be a valid cancer target, as it is not reachable for CAR T cells on healthy colon. For CAR T cell therapy this can be precious information. As healthy cells express certain markers in a polarized fashion this could also be used helping CAR T cells to differentiate between healthy and malignant cells. For example, the family member of CD66c, CEA is also expressed to the luminal side of the colon (Thistlethwaite et al., 2017). This polarity is lost in desmoplastic cancer cells, enabling CAR T cells to target these specifically.

Ultimately, investigators are enabled to not restrain the number of antigens to be measured on rare samples. Especially, healthy tissues are difficult to obtain. During the course of this study, we performed a multi-tissue array of 17 non-malignant tissues and investigated the expression of CD66c, CD318 and TSPAN8 on each, using cyclic IF. We found a very restricted expression of these targets in normal tissue, without using a second slice from any tissue. As the number of investigated antigens can be increased, it is a future option to investigate dozens of markers on multiple tissues simultaneously. While the measurement of multiple antigens on one tumor 
sample could help stratifying patients, the measurement of few markers on multiple healthy tissues facilitates a simplified drug or biomarker discovery.

\subsection{CAR design}

\subsubsection{Safety engineering of CAR T cells}

Even though CLA and CD318 could be targetable by a direct CAR (given a technical method for CLA-CAR T cell generation is established), as introduced in this study, somatic tissue expression - especially of CD66c and TSPAN8 - raises some concern about their applicability as stand-alone targets of conventional CARs and accordingly a strategy to prevent arising toxicities should be prepared. Previously, combinatorial CAR engineering strategies have been developed to mitigate or completely spare off-tumor toxicities. The idea is that CAR T cells are only activated when a target combination is present on the cell. Of course, this combination of targets has to be unique to the malignant cell.

An auspicious combination for this strategy could be the engagement of CLA and CD318 or TSPAN8, as these have the most distinct expression patterns. CLA so far has only been reported to be expressed leucocyte subsets and high endothelial venules (Fuhlbrigge et al., 1997; Strunk et al., 1997; Tu et al., 1999; Yoshino et al., 1999; Dzionek et al., 2000; Tsuchiyama et al., 2002), while this study showed very restricted expression of the two other targets with no overlapping expression on blood cells. This is in contrast to CD66c, which was markedly expressed on neutrophils, therefore bearing the risk of cytotoxic action on neutrophils if CLA is targeted together with CD66c. CD318 in combination with TSPAN8 might be a good combination as well, although overlapping expression in the colon could elicit damage. Finally, a combination of one of the novel target candidates presented here with a target already in clinical investigation, such as mesothelin, could be a promising option, too.

Two approaches to enable these combinatorial targeting are described in the literature. One approach relies on the principle of inducing full activation of the CAR $\mathrm{T}$ cell only when a combination of two target antigens is present on the target cell (Fig. 4). This can be achieved by splitting the main- and co-stimulating domains onto two individual CARs. These CARs are directed to different antigens, thus the requirement for this system to induce activation is the binding of a target combination, uniquely specific to the tumor cells (Fig. 4). Following the termination of Boolean logic, this concept is referred to as AND-gate or AND-approach and has been shown to elicit specific activation (Wilkie et al., 2012; Kloss et al., 2013; Lanitis et al., 2013). Using this concept, full CAR T cell activation has been restricted to cells expressing 
the PDAC antigens HER2 and MUC1 (Wilkie et al., 2012), PSCA and PSMA (Kloss et al., 2013) and MSLN with folate receptor $\alpha$ (Lanitis et al., 2013). These systems using split mainand co-stimulating signal occasionally show activation when only a single target is presented, requiring affinity tuning and further modulations. This approach is currently investigated in a clinical setting using CD19 and a varying set of secondary antigens (NCT03125577).

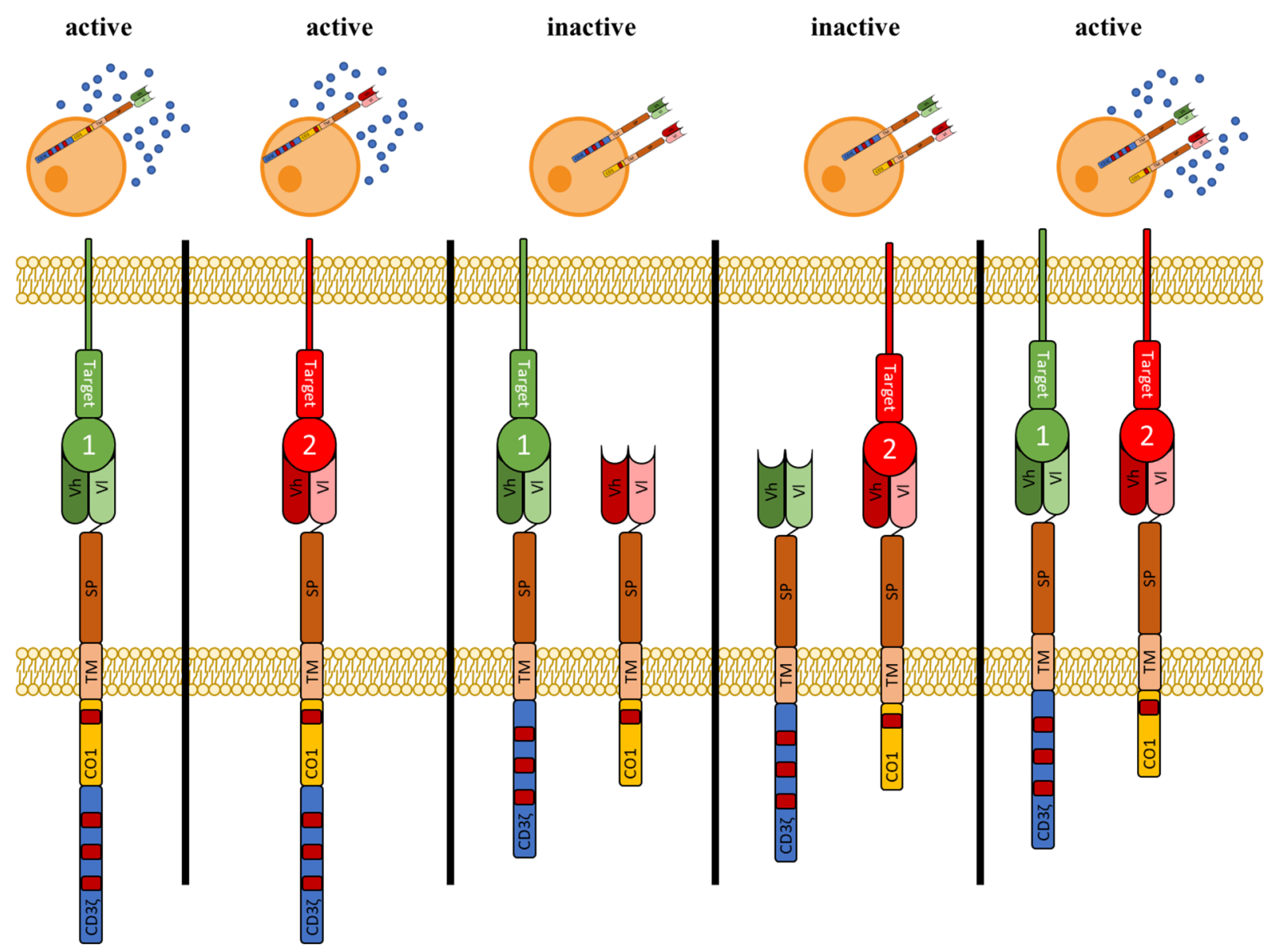

Figure 4: Increasing CAR T cell specificity by applying Boolean AND gates: Part I. From left to right: T cells expressing a second generation CAR are activated upon engaging with an antigen. When the main-stimulatory signal $(\mathrm{CD} 3 \zeta)$ and the co-stimulatory signal (CO1) are split onto two CARs with differing specificity, single engagement of either target antigen 1 (green) or target antigen 2 (red) alone are not sufficient anymore to activate the CAR T cell. If both antigens are present on the target cell, main- and co-stimulatory signals are triggered again simultaneously, leading to a fully activated CAR T cell. scFv heavy chain $\left(\mathrm{V}_{\mathrm{h}}\right)$ and light chain $\left(\mathrm{V}_{1}\right)$. Spacer $(\mathrm{SP})$. Transmembrane domain (TM), Red boxes (ITAM).

A more recent concept also follows the AND-gate logic, but with underlying temporal split mechanism (Fig. 5). The first receptor upon binding target 1 induces the expression of a fully functional CAR construct directed to target 2 (Roybal et al., 2016). This system employs synthetic Notch receptors (synNotch) that have been developed by the very same group before (Morsut et al., 2016). These synNotch receptors recognize tumor antigen 1, but unlike the above mentioned mechanism do not trigger $\mathrm{T}$ cell activation. Instead, a transcription factor is cleaved from the synNotch molecule. This transcription factor localizes to the nucleus and drives 
expression of a CAR construct. This CAR is specific for tumor antigen 2 and now induces T cell activation and cytotoxicity (Fig. 5). While the synNotch system is shown to be not leaky in terms of activation in absence of one antigen, it is relatively stable, raising the concern that $\mathrm{T}$ cells may spread throughout the body and become active in unwanted regions before downregulation of the CAR.

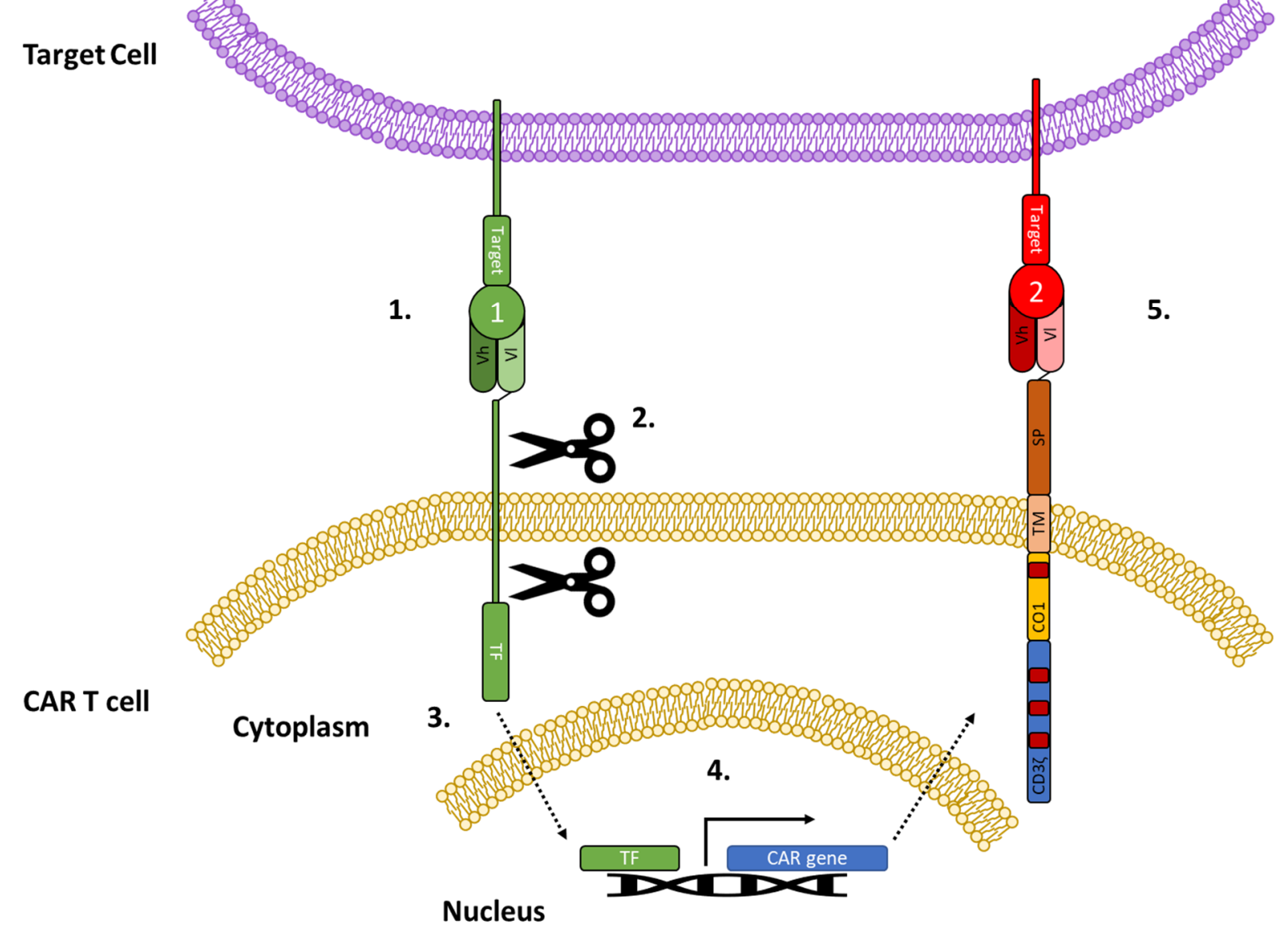

Figure 5: Increasing CAR T cell specificity by applying Boolean AND gates: Part II. The depicted system relies on synthetic Notch (synNotch) receptors (green). 1. An scFv directed to antigen 1 is connected to a Notch receptor. 2. Upon antigen 1 binding, enzymatic cleavage of the Notch receptor domain takes place and a transcription factor (TF) is released into the cytoplasm. 3. The TF diffuses into the nucleus and binds the promotor region of a CAR gene. 4. The CAR is transcribed and translated. 5. The CAR reaches the cell surface and now

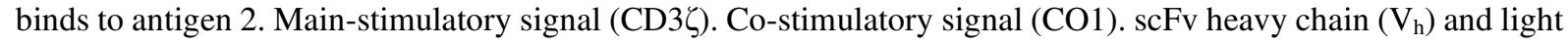
chain $\left(\mathrm{V}_{1}\right)$. Spacer (SP). Transmembrane domain (TM), Red boxes (ITAM).

Another combinatorial option could be overriding of the activation signal by a stronger inhibitory signal triggered by the detection of a second antigen (Fig. 6). These Boolean NOT combination is also known as iCARs (Fedorov et al., 2013). More precisely, the first CAR activates the $\mathrm{T}$ cell upon encounter of an antigen, which is expressed on tumor and healthy cells. On healthy but not tumor cells, a second antigen is expressed. A second CAR binds this antigen, but instead of conveying an activation signaling, this CAR evolves inhibitory signaling, stopping the whole CAR T cell from overcoming the activation threshold (Fig. 6). While this 
principle allows for a rather sharp distinguishing of malignant and healthy tissue, this approach is not well investigated.

In the context of this study, CLA could be used as an activating antigen. However, it is to be expected that toxicities could arise on leukocytes, which could be prevented by an inhibitory anti-CD45 CAR.

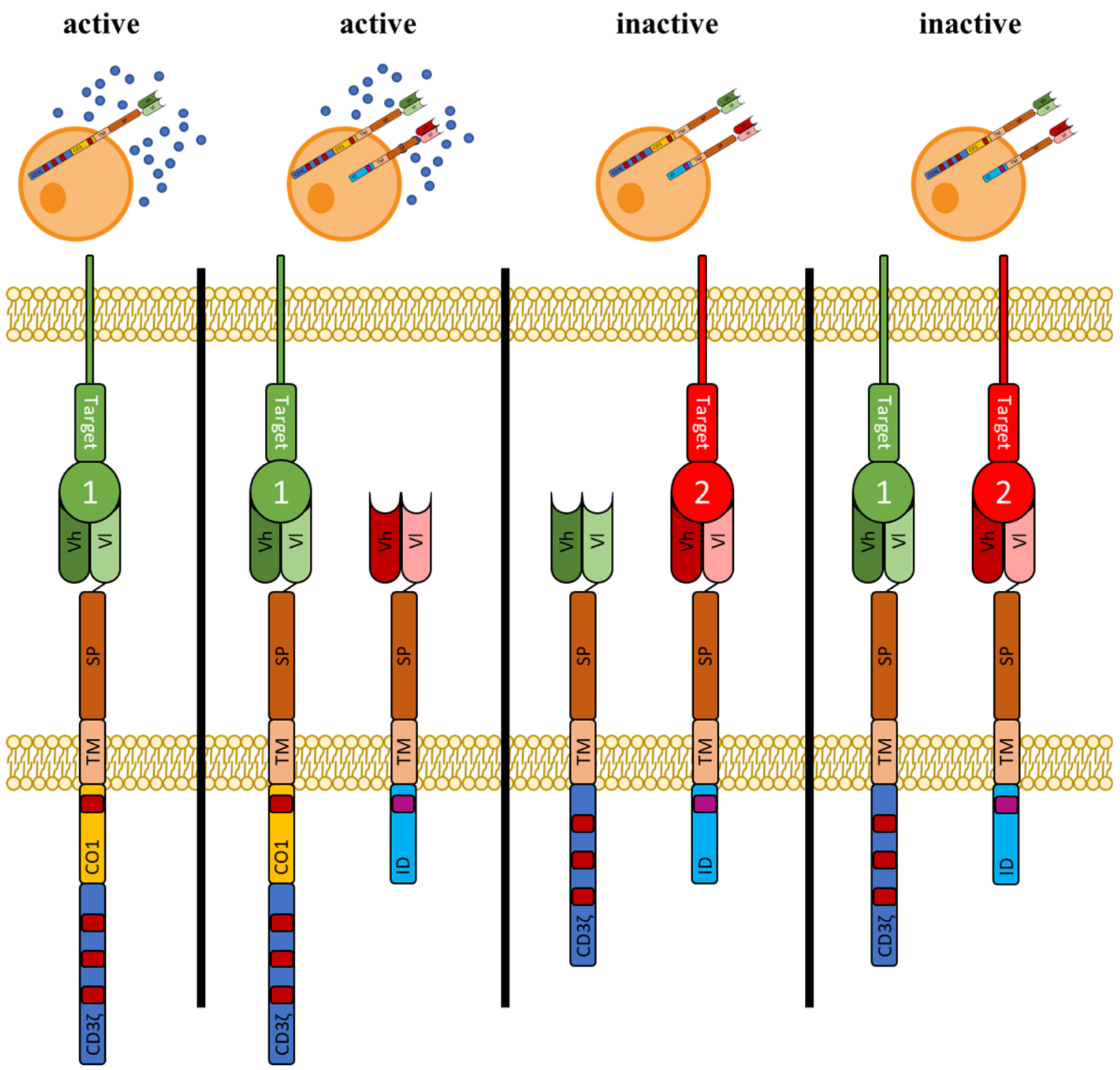

Figure 6: Increasing CAR T cell specificity by applying a Boolean NOT gate. From left to right: $\mathrm{T}$ cells expressing a second generation CAR are activated upon engaging with an antigen. In addition to the second generation CAR directed against antigen 1, an inhibitory CAR (iCAR) specific for antigen 2 is introduced into the

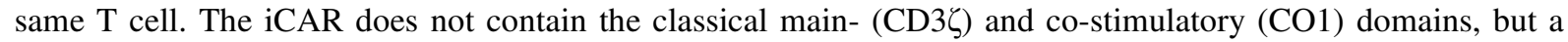
deactivating or inhibitory domain (ID), for example derived from PD-1 or CTLA-4. Whenever antigen 2 is bound, the inhibitory signal overrides any positive signal, thus preventing CAR T cell activation. This mechanism can be applied in order to spare essential healthy cells, that share antigen 1 with the malignant cell. Target antigen 1 (green). Target antigen 2 (red). scFv heavy chain $\left(\mathrm{V}_{\mathrm{h}}\right)$ and light chain $\left(\mathrm{V}_{\mathrm{l}}\right)$. Spacer $(\mathrm{SP})$. Transmembrane domain (TM), Red boxes (ITAM). Violet box (inhibitory region). 


\subsubsection{Rational of CAR design and selection}

We chose a 4-1BB/CD3 $\zeta$ second generation construct with a CD8 $\alpha$ transmembrane domain for our CAR design, as those represent the current clinical standard for PDAC CAR T cell therapies (DeSelm et al., 2017). The variable regions of the light $\left(\mathrm{V}_{\mathrm{l}}\right)$ and heavy chain $\left(\mathrm{V}_{\mathrm{h}}\right)$ were derived from the antibodies used for target discovery and interconnected with a $\left(\mathrm{G}_{4} \mathrm{~S}\right)_{3}$-linker. We constructed each CAR version with two differing $\mathrm{scFv}$ orientations, as it has been shown to affect binding and can lead to tonic signaling (Ajina and Maher, 2018). A common problem encountered with novel target epitopes is missing knowledge about the epitope's position. Previous studies showed that the localization of the target epitope influences CAR function (Guest et al., 2005; Haso et al., 2013; Hudecek et al., 2013; Hudecek et al., 2015). Thus, we created a library of CARs specific for each target, using differing spacer lengths. In total, 34 constructs were designed. In search for the most efficient CAR construct, we examined cytotoxicity, cytokine release and activation marker up-regulation. We found CD66c $\mathrm{V}_{1}-\mathrm{V}_{\mathrm{h}} \mathrm{XS}$, CD318 $\mathrm{V}_{\mathrm{h}}-\mathrm{V}_{1} \mathrm{XS}$, and TSPAN8 $\mathrm{V}_{\mathrm{h}}-\mathrm{V}_{1} \mathrm{~S}$ to be the most effective CAR constructs, with respect to our parameters. However, alternative parameters have been suggested as well, such as proliferation, phenotype or the ability to be stimulated several times (Davenport et al., 2015; Garfall et al., 2019). In fact, some of our chosen parameters might be correlated with disadvantageous clinical outcome. High cytokine release can lead to the cytokine release syndrome (CRS), one of the most severe side effects of CAR therapy (Brudno and Kochenderfer, 2016). Too strong T cell activation can lead to AICD and exhaustion (Künkele et al., 2015). Thus, it has to be evaluated, if this CAR selection process can also deliver the clinically most beneficial CAR construct. Nonetheless, our best performing CARs in vitro were also the best performing ones in vivo proving the applied strategy to be useful.

\subsubsection{Influence of the CAR spacer on CAR T cell functionality}

During our experimental in vitro assessment of the varying CAR constructs, it became apparent that the XS and S spacer performed almost indistinguishable good in the CD66c and CD318 setting during the pre-assays, while TSPAN8 directed CARs performed best in S and L spacer configuration. Surprisingly, in the final assay comparing only CD66c XS and S spacer CAR, the XS spacer CAR showed no activity, although it has been functional before. The subsequent decision to perform in vivo experiments only with the $\mathrm{S}$ spacer was based on this experiment. A retrospective analysis however revealed a low transduction efficacy, making it likely, that the CD66c XS spacer CAR is still a potent CAR. Indeed, this was confirmed in a subsequent in vivo trial, which was not part of this study. These observations underline the hypothesis of an 
optimal distancing between effector and target cell, as indicated by previous studies (Guest et al., 2005; James et al., 2008; Haso et al., 2013; Hudecek et al., 2013; Krenciute et al., 2016). This may be caused by the natural steric circumstances of $\mathrm{T}$ cell activation.

The minimal components for T cell activation by natural TCR:MHC binding are well studied. The TCR binding to the MHC complex triggers the formation of a rigid and well-structured immunological synapse (Monks et al., 1998; Freiberg et al., 2002). This synapse is subdivided in three major supramolecular activation clusters (SMACs): central SMAC (cSMAC), peripheral (pSMAC) and distal (dSMAC). The TCR accumulates in the cSMAC, while in the dSMAC cell-cell attachment proteins cluster, like LFA-1 or CD2. In dSMAC, inhibitory phosphatases such as CD45 and CD148 are situated, which would otherwise prevent TCR/CD3 phosphorylation (Onnis and Baldari, 2019). Each SMAC region on its own is dependent on defined distances between the antigen presenting cell and effector cell. In the central region, the distance is commonly $15 \mathrm{~nm}$ as dictated by the MHC:TCR complex (Garcia et al., 1996). Although a CAR synapse is less structured and seems to follow partially different requirements for activation, the distancing between CAR and epitope seems to generate higher activity when certain distance requirements are fulfilled (Guest et al., 2005; Haso et al., 2013; Hudecek et al., 2013; Hudecek et al., 2015). This could be explained by the necessity of the CAR synapse to recapitulate the $15 \mathrm{~nm}$ distance of the TCR:MHC complex, in order to exclude deactivating phosphatases like CD45 and CD148, which both have longer extracellular stalks (Irles et al., 2003; Cordoba et al., 2013). Although previous studies elaborated the importance of spacer length modulation in dependence to the target epitope, less diligence has been dedicated to clarify this issue.

Although the exact position of the respective epitopes in our case is not known, some logical deductions can be performed (Fig. 7). The proteins of interest in this study contain either one extracellular single tail (CD66c and CD318) or two extracellular loops (TSPAN8) (The UniProt Consortium, 2019). The extracellular tail of CD318 consists of 638 amino acids (AAs) making it the longest peptide chain of the three targets. CD66c with 320 AAs has around half the amount of AAs. The extracellular loops of TSPAN8 span only 24 and 96 AAs and the bigger loop has interconnecting disulfide bridges further reducing the extent of the loop. As pure AAs numbers do not allow for reliable conclusions of a three-dimensional protein structure, the assumptions need to be further specified. The extracellular domains of CD66c are two C2-set Ig-like domains and one V-set Ig-like domain. All Ig-like proteins share a common structural basis. As the size is known to be approximately $5 \mathrm{~nm}$ from the constant heavy chain $(\mathrm{CH}) \mathrm{Ig}$ domains 
from antibodies (Harris et al., 1998; Ryazantsev et al., 2013; Wolak et al., 2015), it can be assumed that the total extracellular size of CD66c should be $\sim 15 \mathrm{~nm}$. Furthermore, it is known for the CD66c antibody used in this study, that the binding epitope is situated on the outer variable $\mathrm{V}$-set domain. In conclusion, TSPAN8 represents a very membrane proximal target, while the CD66c epitope is located in approximately $15 \mathrm{~nm}$ distance from the membrane. For CD318, no estimation is applicable, however based on its size it is rather likely to have a membrane distal epitope. These different sized targets were now opposed by CAR constructs with varying spacer lengths. The spacers used were based on the human IgG4 hinge from the CH2-CH3 domains (L; long spacer; 228 AAs; 10 nm), the human IgG4 hinge from the CH3 domain (medium spacer; $119 \mathrm{AAs}, \sim 5 \mathrm{~nm}$ ), the human IgG4 hinge from the sequence between $\mathrm{CH} 2$ and $\mathrm{CH} 1$ domain (XS; extra short spacer; $12 \mathrm{AAs}$ ) or a human CD8 $\alpha$ domain (S; short spacer; 45 AAs).

As outlined before, the presumably ideal distance between a CAR T cell and target cell is $\sim 15 \mathrm{~nm}$ and the CD66c epitope might be $\sim 15 \mathrm{~nm}$ distal from the membrane. Not surprisingly, the shortest CAR performed well, while the almost $15 \mathrm{~nm}$ spanning L spacer was inactive. Conversely, this spacer performed very good on the membrane-proximal TSPAN8, while the short spacer was dysfunctional in this setting. Of note, also surface protein CD318 was best targeted with the XS and S spacer.

Why is the CD8 $\alpha$-derived S spacer able to engage both distal and proximal targets? A potential explanation for this phenomenon could be the structural nature of the $\mathrm{CD} 8 \alpha$ spacer. For $\mathrm{CH}$ domain based IgG spacers, crystal structures are available. Their size is well known, forming a rigid three-dimensional structure. This is in contrast to the CD8 $\alpha$ derived spacer. This spacer is derived from the stalk of the CD8 $\alpha$ molecule, of which no structural information are available (Leahy et al., 1992). This is caused by the flexible nature of the stalk, making it impossible to crystallize. This fact may be the explanation for the versatility of this spacer by being flexible enough to convey binding over a broad range of distances.

However, these explanations are based on cytotoxicity experiments and deduction from known sizes of related molecules. A real experimental proof for the mechanisms is missing. Future studies in this direction should focus less on effector functionality and more on the inter-cell distancing. High-resolution microcopy or electron microscopy would be suitable techniques to elucidate the distance relations between $\mathrm{T}$ cell, target cell and spacer. 


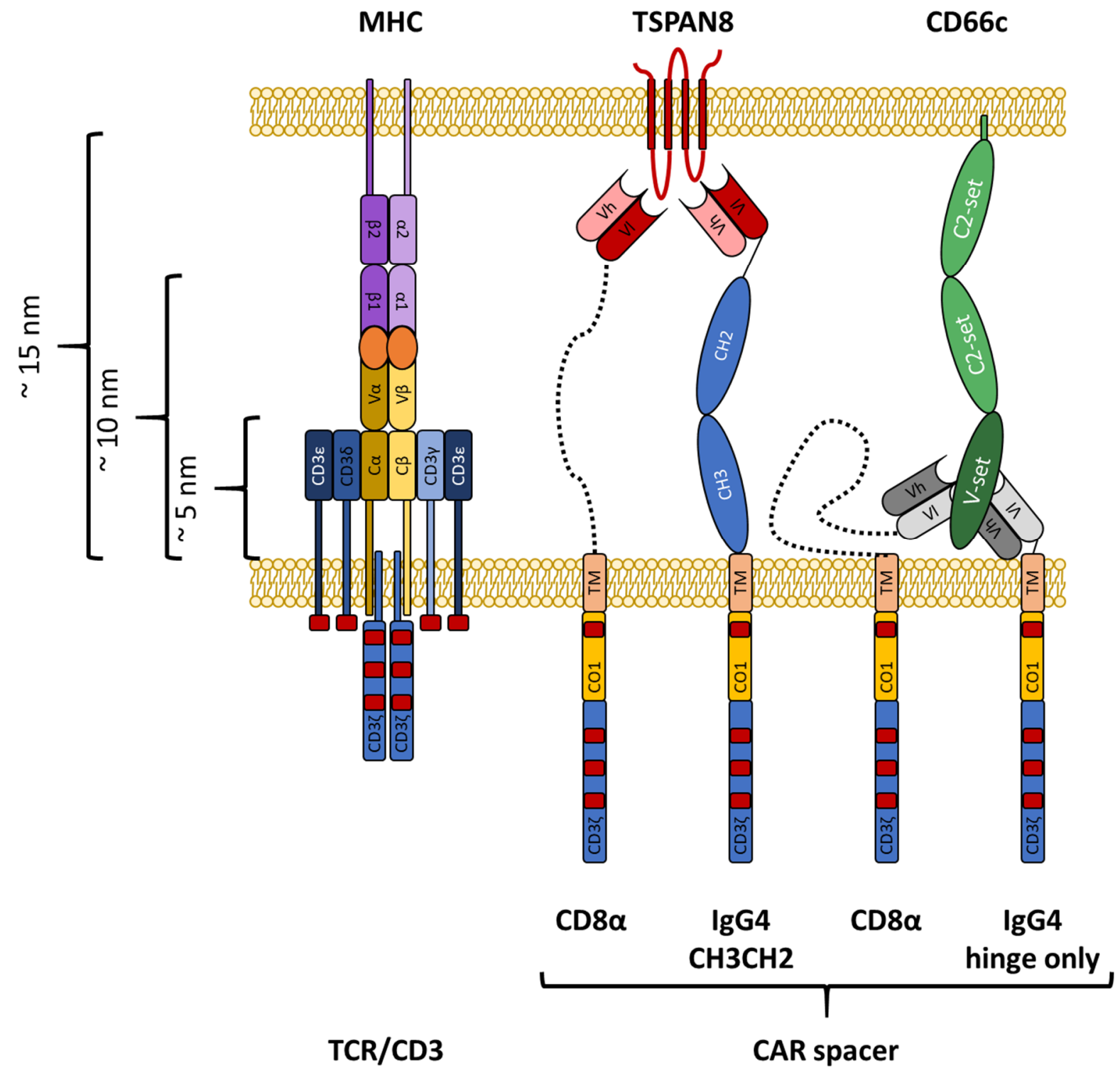

Figure 7: Hypothetical steric CAR:antigen interactions at a CAR synapse. The distance in a natural occurring immunological synapse is at the center supramolecular activation cluster (cSMAC) given through the steric interactions of the TCR:MHC complex. The distance of this complex is known to be approximately $15 \mathrm{~nm}$ (Garcia et al., 1996). This is important for T cell activation, as deactivating phosphatases like CD45 and CD148 are excluded from the cSMAC because their extracellular stalks are longer than these $15 \mathrm{~nm}$. Hence, they are dispelled to regions with more space such as distal SMAC (Irles et al., 2003; Cordoba et al., 2013). This suggests the need of a CAR synapse to roughly recapitulate the steric situation of a natural occurring immunological synapse, in order to exclude CD45 and CD148. The size of the C-set Ig-like constant region domains (CH) of antibodies is also known to be approximately $5 \mathrm{~nm}$ (Harris et al., 1998; Ryazantsev et al., 2013; Wolak et al., 2015). Thus, a long IgG4 CH3-CH2 spacer can span $\sim 10 \mathrm{~nm}$, leaving $\sim 5 \mathrm{~nm}$ for the $\mathrm{scFv}$ antigen interaction to match the optimal $15 \mathrm{~nm}$. In favor of this assumption are the efficacy data showing that only long TSPAN8 CARs activate T cells efficiently. CD66c consists of two C2-set domains and one V-set domain, the former ones being structural relatives of the $\mathrm{CH}$ domains. It can thus be assumed that they also extend to $\sim 5 \mathrm{~nm}$. As the epitope for the CD66c scFv is located on the membrane distal V-set domain, a CAR spacer needs to be short to recapitulate the $15 \mathrm{~nm}$. Indeed, the long CAR spacers were not efficient at targeting CD66c. An exception is the CD8 $\alpha$ spacer. CD8 $\alpha$ spacer CARs perform well on membrane proximal and distal targets. Unsurprisingly, the CD8 $\alpha$ stalk is known to be flexible (Leahy et al., 1992), therefore allowing binding of a wider range of epitope positions. 


\subsubsection{A new class of Siglec derived CAR spacers}

When evaluated in our murine in vivo PDAC model, the L spacer CAR directed to TSPAN8 only controlled tumor growth, while the S spacer CAR completely eradicated the tumors. This may be due to unspecific binding, as previous studies pointed out that unspecific binding of IgG1 and IgG4 based long spacers to Fc-receptors can hinder T cell function (Hombach et al., 2010; Hudecek et al., 2015; Jonnalagadda et al., 2015). CH2 domains contain a binding moiety for Fc-receptors. However, $\mathrm{T}$ cell function can be rescued by mutating the binding regions, thus preventing unspecific interactions (Hombach et al., 2010; Hudecek et al., 2015; Jonnalagadda et al., 2015). Our IgG4 derived long spacer contained the very same mutations, which raised doubt if all unspecific interactions are abolished. We showed that PDAC xenograft tumors in NSG mice were well infiltrated by murine $\mathrm{CD}^{+} 8^{+}$macrophages, leaving the possibility of some remaining interactions between these murine immune cells and the CAR T cells. This concern was further nurtured by the complete failure of CAR T cell activity when we used a long IgG1 spacer CAR in another experiment. While a CD20 targeting CAR based on a long IgG1 spacer, also containing previously reported mutations to abolish FcR binding, was indistinguishable effective from the S spacer based CAR on $\mathrm{CD} 20^{+}$lymphoma cells in vitro, it did not display efficacy in the subsequent in vivo study. This was even more surprising, considering that CD20 is a membrane proximal target. Of note, no commercially approved CAR T cell product as of today is based on an IgG derived spacer.

Due to these limitations, we aimed at creating a novel class of CAR spacer, having similar attributes as compared to $\operatorname{IgG}$ class spacer and analogous modularity. We screened naturally occurring protein families of immune cells and identified the Sialic acid-binding immunoglobulin-type lectin (Siglec) family as an auspicious alternative. Siglec molecules span a varying number of C2-set Ig-like domains between the membrane and the extracellular $\mathrm{V}$-set binding domain (Macauley et al., 2014; Bornhöfft et al., 2018). However, in contrast to IgG, they naturally do not carry a FcR binding moiety, and - without few exceptions - are not known to convey binding offside the $\mathrm{V}$-set domain.

Alternative families with similar attributes that could have been considered as well, are the CEA family or Signaling lymphocytic activation molecule (SLAM) family. They also consist of differing numbers of C-set domains, have a V-set domain on the distal end and can thus be modulated, and are expressed on immune cells (Hammarström, 1999; Dragovich and Mor, 2018). 
We selected one, two or three C2-set domains derived from Siglec-3, -4, -7, or -8 for generation of the new spacer library. One to three $\mathrm{C}$-set domains have also been readily investigated in the context of IgG based spacer (Faitschuk et al., 2016; Watanabe et al., 2016). From the five CARs designed, only Siglec-4, Siglec -7.2 and Siglec -8 spacer CARs were found to be expressed on $\mathrm{T}$ cells. The Siglec-4 spacer CAR was singled out as the most effective one in co-culture experiments using CD $20^{+}$target cells and CD20 targeting CARs. Based on this finding, it was chosen for further investigation. However, the other two CARs - comprising Siglec-7.2 and Siglec-8 spacer - contained only one C2-set domain. Knowing about the optimal distancing between CAR T and target cell, this was a suboptimal setting as the CD20 antigen is located very membrane proximal. The very good performance of the Siglec-4 spacer CAR could be reproduced in a PDAC tumor mouse model, using a different membrane proximal target, namely TSPAN8. At the same time, this CAR was not able to develop a cytotoxic response when targeting CD66c, again supporting the model discussed above. Subsequent studies could either include Siglec-7.2 or Siglec-8 spacer CARs or Siglec-4 spacer CARs with only one C2set domain for targeting CD66c. While we could show that spacers derived from the Siglec family are effective CAR spacers, we did not prove the modularity of the spacer, meaning the adjustability towards targets with differing distances to the membrane. Future studies should elaborate these open questions to complete the toolbox of this new spacer family.

Finally, the novel Siglec-4 spacer CARs induced in vitro a more beneficial cytokine release pattern and in vivo a phenotypic cell profile shifted towards the central memory $\mathrm{T}$ cell compartment as compared to the CD8 $\alpha$ based CARs. This might have promising clinical implications, as massive cytokine release upon activation is one of the most severe side effects of current CAR therapies and an equally efficient CARs with lower cytokine release would decrease this risk for patients (Brudno and Kochenderfer, 2016). In addition, central memory T cells have been shown to convey a superior anti-tumor performance in contrast to cells shifted more to the effector compartment (Liu et al., 2020). Although promising, the underlying mechanisms are not yet understood, which is why these results need to be corroborated also in other target and indication settings to evaluate, if these beneficial traits can be retained. 


\subsection{Conclusion and outlook}

The first two prospects of this study were the identification of novel target antigens for CAR based cellular therapy of PDAC with a safe off-tumor expression profile and the generation of functional CARs directed towards these novel target candidates. Four target candidates, namely CLA, CD318, CD66c and TSPAN8, were identified showing high tumor specificity and very restricted off-tumor expression. Although they appear with more favorable expression patterns than most other PDAC CAR targets, not all concerns could be resolved. An effective targeting of CLA requires the development of processes facilitating a functional knock-out of this glycosylation, while simultaneously preserving sufficient $\mathrm{T}$ cell transduction. Whereas CARs directed to CLA face technical roadblocks, CARs specific for CD318, CD66c and TSPAN8 were able to evolve potent anti-tumor activity, both in vitro and in vivo. However, CD66c and TSPAN8 exhibit some limited but specific somatic-tissue expression, which could lead to toxicities when unmodified direct CAR T cells are administrated. This implies the necessity for further developments on strategies helping to avoid unwanted side-effects, in particular ontarget off-tumor toxicity. Advanced CAR engineering would be a promising option, in particular combinatorial logic gates, such as AND and NOT CARs. The usage of target combinations would further elevate CAR T cell specificity and the targets presented herein display normal tissue expression patterns suitable for such combinatorial approaches.

This study showed that spacer modulation can induce significant differences regarding CAR activation, representing a valid option for CAR fine-tuning. The newly developed Siglec-4 derived spacer bears the potential to help decreasing the risk of cytokine storm, possibly enabling to widen therapeutic window, which is a beneficial trait for potential clinical applications in the future. Whether this option is only available with the full-length spacer on membrane proximal targets or additionally on more distal epitopes, in case $\mathrm{C} 2$-set domains are removed, needs to be elucidated. Future studies addressing spacer related topics, would ideally make use of techniques enabling a direct read-out of the parameters of interest. High-resolution microcopy techniques are suitable to analyze distances between $\mathrm{T}$ cell and tumor cell or even interaction of molecules within a CAR synapse. A comprehensive knowledge of interactions among molecules involved in CAR synapse formation will help to advance our understanding on how CAR structural elements such as the spacer domains influence CAR functionality. This study describes four novel target candidates for CAR based cellular therapy of PDAC together with a set of highly functional CAR constructs. The thorough evaluation of somatic tissue expression will help to estimate and manage potential toxicities arising during clinical translation or even preventing those by next generation CAR design based on co-expression 
patterns. In addition, a novel class of Siglec protein family derived spacers was introduced, with the Siglec-4 spacer exhibiting beneficial traits for potential clinical applications in the future. 


\section{References}

Abate-Daga, D., Lagisetty, K.H., Tran, E., Zheng, Z., Gattinoni, L., Yu, Z., et al. (2014). A Novel Chimeric Antigen Receptor Against Prostate Stem Cell Antigen Mediates Tumor Destruction in a Humanized Mouse Model of Pancreatic Cancer. Human Gene Therapy 25(12), 1003-1012. doi: 10.1089/hum.2013.209.

Ahmed, N., Brawley, V.S., Hegde, M., Robertson, C., Ghazi, A., Gerken, C., et al. (2015). Human Epidermal Growth Factor Receptor 2 (HER2) -Specific Chimeric Antigen Receptor-Modified T Cells for the Immunotherapy of HER2-Positive Sarcoma. Journal of clinical oncology : official journal of the American Society of Clinical Oncology 33(15), 1688-1696. doi: 10.1200/JCO.2014.58.0225.

Ajina, A., and Maher, J. (2018). Strategies to Address Chimeric Antigen Receptor Tonic Signaling. Molecular cancer therapeutics 17(9), 1795-1815. doi: 10.1158/15357163.MCT-17-1097.

Akce, M., Zaidi, M.Y., Waller, E.K., El-Rayes, B.F., and Lesinski, G.B. (2018). The Potential of CAR T Cell Therapy in Pancreatic Cancer. Frontiers in Immunology 9(2166). doi: 10.3389/fimmu.2018.02166.

Almoguera, C., Shibata, D., Forrester, K., Martin, J., Arnheim, N., and Perucho, M. (1988). Most human carcinomas of the exocrine pancreas contain mutant c-Kras genes. Cell 53(4), 549-554. doi: 10.1016/0092-8674(88)90571-5.

Angerer, P., Simon, L., Tritschler, S., Wolf, F.A., Fischer, D., and Theis, F.J. (2017). Single cells make big data: New challenges and opportunities in transcriptomics. Current Opinion in Systems Biology 4, 85-91. doi: https://doi.org/10.1016/j.coisb.2017.07.004.

Bailey, P., Chang, D.K., Nones, K., Johns, A.L., Patch, A.M., Gingras, M.C., et al. (2016). Genomic analyses identify molecular subtypes of pancreatic cancer. Nature 531(7592), 47-52. doi: 10.1038/nature16965.

Barber, A., Zhang, T., Megli, C.J., Wu, J., Meehan, K.R., and Sentman, C.L. (2008). Chimeric NKG2D receptor-expressing T cells as an immunotherapy for multiple myeloma. Exp Hematol 36(10), 1318-1328. doi: 10.1016/j.exphem.2008.04.010.

Barrett, T., Wilhite, S.E., Ledoux, P., Evangelista, C., Kim, I.F., Tomashevsky, M., et al. (2012). NCBI GEO: archive for functional genomics data sets-update. Nucleic Acids Research 41(D1), D991-D995. doi: 10.1093/nar/gks1193.

Bausch-Fluck, D., Goldmann, U., Müller, S., van Oostrum, M., Müller, M., Schubert, O.T., et al. (2018). The in silico human surfaceome. Proceedings of the National Academy of Sciences 115(46), E10988-E10997. doi: 10.1073/pnas.1808790115.

Beatty, G.L., O'Hara, M.H., Lacey, S.F., Torigian, D.A., Nazimuddin, F., Chen, F., et al. (2018). Activity of Mesothelin-Specific Chimeric Antigen Receptor T Cells Against Pancreatic Carcinoma Metastases in a Phase 1 Trial. Gastroenterology 155(1), 29-32. doi: 10.1053/j.gastro.2018.03.029.

Behrens, G., Jochem, C., Schmid, D., Keimling, M., Ricci, C., and Leitzmann, M.F. (2015). Physical activity and risk of pancreatic cancer: a systematic review and meta-analysis. Eur J Epidemiol 30(4), 279-298. doi: 10.1007/s10654-015o014-9.

Bezbradica, J.S., and Medzhitov, R. (2012). Role of ITAM signaling module in signal integration. Curr Opin Immunol 24(1), 58-66. doi: 10.1016/j.coi.2011.12.010.

Bilimoria, K.Y., Bentrem, D.J., Ko, C.Y., Stewart, A.K., Winchester, D.P., and Talamonti, M.S. (2007). National failure to operate on early stage pancreatic cancer. Annals of surgery 246(2), 173-180. doi: 10.1097/SLA.obo13e3180691579. 
Bornhöfft, K.F., Goldammer, T., Rebl, A., and Galuska, S.P. (2018). Siglecs: A journey through the evolution of sialic acid-binding immunoglobulin-type lectins. Dev Comp Immunol 86, 219-231. doi: 10.1016/j.dci.2018.05.008.

Bosetti, C., Bravi, F., Turati, F., Edefonti, V., Polesel, J., Decarli, A., et al. (2013). Nutrient-based dietary patterns and pancreatic cancer risk. Ann Epidemiol 23(3), 124-128. doi: 10.1016/j.annepidem.2012.12.005.

Bosetti, C., Lucenteforte, E., Silverman, D.T., Petersen, G., Bracci, P.M., Ji, B.T., et al. (2012). Cigarette smoking and pancreatic cancer: an analysis from the International Pancreatic Cancer Case-Control Consortium (Panc4). Ann Oncol 23(7), 1880-1888. doi: 10.1093/annonc/mdr541.

Bosetti, C., Rosato, V., Li, D., Silverman, D., Petersen, G.M., Bracci, P.M., et al. (2014). Diabetes, antidiabetic medications, and pancreatic cancer risk: an analysis from the International Pancreatic Cancer Case-Control Consortium. Annals of oncology : official journal of the European Society for Medical Oncology 25(10), 2065-2072. doi: 10.1093/annonc/mdu276.

Bosse, K.R., Raman, P., Zhu, Z., Lane, M., Martinez, D., Heitzeneder, S., et al. (2017). Identification of GPC2 as an Oncoprotein and Candidate Immunotherapeutic Target in High-Risk Neuroblastoma. Cancer cell 32(3), 295-309.e212. doi: 10.1016/j.ccell.2017.08.003.

Bramhall, S.R., Allum, W.H., Jones, A.G., Allwood, A., Cummins, C., and Neoptolemos, J.P. (1995). Treatment and survival in 13,560 patients with pancreatic cancer, and incidence of the disease, in the West Midlands: an epidemiological study. Br J Surg 82(1), 111-115. doi: 10.1002/bjs.1800820137.

Brembeck, F.H., Schreiber, F.S., Deramaudt, T.B., Craig, L., Rhoades, B., Swain, G., et al. (2003). The mutant K-ras oncogene causes pancreatic periductal lymphocytic infiltration and gastric mucous neck cell hyperplasia in transgenic mice. Cancer Res 63(9), 2005-2009.

Brentjens, R.J., Santos, E., Nikhamin, Y., Yeh, R., Matsushita, M., La Perle, K., et al. (2007). Genetically targeted T cells eradicate systemic acute lymphoblastic leukemia xenografts. Clin Cancer Res $13(18$ Pt 1), 5426-5435. doi: 10.1158/1078-0432.ccr-07-0674.

Bridgeman, J.S., Hawkins, R.E., Bagley, S., Blaylock, M., Holland, M., and Gilham, D.E. (2010). The Optimal Antigen Response of Chimeric Antigen Receptors Harboring the CD3 $\zeta$ Transmembrane Domain Is Dependent upon Incorporation of the Receptor into the Endogenous TCR/CD3 Complex. The Journal of Immunology, ji_o901766. doi: 10.4049/jimmunol.0901766.

Brocker, T. (2000). Chimeric Fv-zeta or Fv-epsilon receptors are not sufficient to induce activation or cytokine production in peripheral T cells. Blood 96(5), 1999-2001.

Brocker, T., and Karjalainen, K. (1998). "Adoptive Tumor Immunity Mediated by Lymphocytes Bearing Modified Antigen-Specific Receptors," in Advances in Immunology, ed. F.J. Dixon. Academic Press), 257-269.

Brudno, J.N., and Kochenderfer, J.N. (2016). Toxicities of chimeric antigen receptor T cells: recognition and management. Blood 127(26), 3321-3330. doi: 10.1182/blood-2016-04-703751.

Burris, H.A., 3rd, Moore, M.J., Andersen, J., Green, M.R., Rothenberg, M.L., Modiano, M.R., et al. (1997). Improvements in survival and clinical benefit with gemcitabine as first-line therapy for patients with advanced pancreas cancer: a randomized trial. $J$ Clin Oncol 15(6), 2403-2413. doi: 10.1200/jco.1997.15.6.2403. 
Cancer Genome Atlas Research Network. Electronic address, a.a.d.h.e., and Cancer Genome Atlas Research, N. (2017). Integrated Genomic Characterization of Pancreatic Ductal Adenocarcinoma. Cancer cell 32(2), 185-203.e113. doi: 10.1016/j.ccell.2017.07.007.

Canon, J., Rex, K., Saiki, A.Y., Mohr, C., Cooke, K., Bagal, D., et al. (2019). The clinical KRAS(G12C) inhibitor AMG 510 drives anti-tumour immunity. Nature 575(7781), 217-223. doi: 10.1038/s41586-019-1694-1.

Carpelan-Holmström, M., Nordling, S., Pukkala, E., Sankila, R., Lüttges, J., Klöppel, G., et al. (2005). Does anyone survive pancreatic ductal adenocarcinoma? A nationwide study re-evaluating the data of the Finnish Cancer Registry. Gut 54(3), 385-387. doi: 10.1136/gut.2004.047191.

Cartellieri, M., Bachmann, M., Feldmann, A., Bippes, C., Stamova, S., Wehner, R., et al. (2010). Chimeric antigen receptor-engineered T cells for immunotherapy of cancer. $J$ Biomed Biotechnol 2010, 956304. doi: 10.1155/2010/956304.

Caruana, I., Savoldo, B., Hoyos, V., Weber, G., Liu, H., Kim, E.S., et al. (2015). Heparanase promotes tumor infiltration and antitumor activity of CARredirected $\mathrm{T}$ lymphocytes. Nature Medicine 21(5), 524-529. doi: $10.1038 / \mathrm{nm} .3833$.

Caruso, H.G., Hurton, L.V., Najjar, A., Rushworth, D., Ang, S., Olivares, S., et al. (2015). Tuning Sensitivity of CAR to EGFR Density Limits Recognition of Normal Tissue While Maintaining Potent Antitumor Activity. Cancer Res 75(17), 35053518. doi: 10.1158/ooo8-5472.can-15-0139.

Charrin, S., Jouannet, S., Boucheix, C., and Rubinstein, E. (2014). Tetraspanins at a glance. Journal of Cell Science 127(17), 3641-3648. doi: 10.1242/jcs.154906.

Chen, H., Yang, G., Xiao, J., Zheng, L., You, L., and Zhang, T. (2020). Neoantigenbased immunotherapy in pancreatic ductal adenocarcinoma (PDAC). Cancer Letters 490, 12-19. doi: https://doi.org/10.1016/j.canlet.2020.06.011.

Chmielewski, M., and Abken, H. (2017). CAR T Cells Releasing IL-18 Convert to TBet(high) FoxO1(low) Effectors that Exhibit Augmented Activity against Advanced Solid Tumors. Cell Rep 21(11), 3205-3219. doi: 10.1016/j.celrep.2017.11.063.

Cleveland, M.H., Sawyer, J.M., Afelik, S., Jensen, J., and Leach, S.D. (2012). Exocrine ontogenies: on the development of pancreatic acinar, ductal and centroacinar cells. Semin Cell Dev Biol 23(6), 711-719. doi: 10.1016/j.semcdb.2012.06.008.

Coman, C., Solari, F.A., Hentschel, A., Sickmann, A., Zahedi, R.P., and Ahrends, R. (2016). Simultaneous Metabolite, Protein, Lipid Extraction (SIMPLEX): A Combinatorial Multimolecular Omics Approach for Systems Biology. Molecular \&amp; Cellular Proteomics 15(4), 1453-1466. doi: 10.1074/mcp.M115.053702.

Conroy, T., Desseigne, F., Ychou, M., Bouché, O., Guimbaud, R., Bécouarn, Y., et al. (2011). FOLFIRINOX versus gemcitabine for metastatic pancreatic cancer. $N$ Engl J Med 364(19), 1817-1825. doi: 10.1056/NEJMoa1011923.

Conroy, T., Hammel, P., Hebbar, M., Ben Abdelghani, M., Wei, A.C., Raoul, J.L., et al. (2018). FOLFIRINOX or Gemcitabine as Adjuvant Therapy for Pancreatic Cancer. N Engl J Med 379(25), 2395-2406. doi: 10.1056/NEJMoa1809775.

Cooper, L.J., Topp, M.S., Serrano, L.M., Gonzalez, S., Chang, W.C., Naranjo, A., et al. (2003). T-cell clones can be rendered specific for CD19: toward the selective augmentation of the graft-versus-B-lineage leukemia effect. Blood 101(4), 16371644. doi: 10.1182/blood-2002-07-1989.

Cordoba, S.-P., Choudhuri, K., Zhang, H., Bridge, M., Basat, A.B., Dustin, M.L., et al. (2013). The large ectodomains of CD45 and CD148 regulate their segregation 
from and inhibition of ligated T-cell receptor. Blood 121(21), 4295-4302. doi: 10.1182/blood-2012-07-442251.

Costa, R.L.B., and Czerniecki, B.J. (2020). Clinical development of immunotherapies for HER2+ breast cancer: a review of HER2-directed monoclonal antibodies and beyond. npj Breast Cancer 6(1), 10. doi: 10.1038/s41523-020-0153-3.

Darcy, P.K., Kershaw, M.H., Trapani, J.A., and Smyth, M.J. (1998). Expression in cytotoxic $\mathrm{T}$ lymphocytes of a single-chain anti-carcinoembryonic antigen antibody. Redirected Fas ligand-mediated lysis of colon carcinoma. Eur $J$ Immunol 28(5), 1663-1672. doi: 10.1002/(sici)15214141(199805)28:05<1663::aid-immu1663>3.0.co;2-l.

Davenport, A.J., Jenkins, M.R., Cross, R.S., Yong, C.S., Prince, H.M., Ritchie, D.S., et al. (2015). CAR-T Cells Inflict Sequential Killing of Multiple Tumor Target Cells. Cancer Immunology Research 3(5), 483-494. doi: 10.1158/2326-6066.cir-150048.

DeSelm, C., Tano, Z., Varghese, A., and Adusumilli, P. (2017). CAR T-cell therapy for pancreatic cancer. Journal of surgical oncology 116. doi: 10.1002/jso.24627.

Diener, M.K., Fitzmaurice, C., Schwarzer, G., Seiler, C.M., Hüttner, F.J., Antes, G., et al. (2014). Pylorus-preserving pancreaticoduodenectomy (pp Whipple) versus pancreaticoduodenectomy (classic Whipple) for surgical treatment of periampullary and pancreatic carcinoma. The Cochrane database of systematic reviews 11(11), CDoo6053-CDo06053. doi: 10.1002/14651858.CDoo6o53.pub5.

Dotti, G., Gottschalk, S., Savoldo, B., and Brenner, M.K. (2014). Design and development of therapies using chimeric antigen receptor-expressing $\mathrm{T}$ cells. Immunological Reviews 257(1), 107-126. doi: 10.1111/imr.12131.

Dragovich, M.A., and Mor, A. (2018). The SLAM family receptors: Potential therapeutic targets for inflammatory and autoimmune diseases. Autoimmunity reviews 17(7), 674-682. doi: 10.1016/j.autrev.2018.01.018.

Duong, C.P., Westwood, J.A., Yong, C.S., Murphy, A., Devaud, C., John, L.B., et al. (2013). Engineering T cell function using chimeric antigen receptors identified using a DNA library approach. PLoS One 8(5), e63037. doi: 10.1371/journal.pone.0063037.

Dwivedi, A., Karulkar, A., Ghosh, S., Rafiq, A., and Purwar, R. (2018). Lymphocytes in Cellular Therapy: Functional Regulation of CAR T Cells. Front Immunol 9, 3180. doi: 10.3389/fimmu.2018.03180.

Dzionek, A., Fuchs, A., Schmidt, P., Cremer, S., Zysk, M., Miltenyi, S., et al. (2000). BDCA-2, BDCA-3, and BDCA-4: Three Markers for Distinct Subsets of Dendritic Cells in Human Peripheral Blood. The Journal of Immunology 165(11), 60376046. doi: 10.4049/jimmunol.165.11.6037.

Eshhar, Z., Waks, T., Bendavid, A., and Schindler, D.G. (2001). Functional expression of chimeric receptor genes in human T cells. J Immunol Methods 248(1-2), 6776. doi: 10.1016/soo22-1759(00)00343-4.

Eshhar, Z., Waks, T., Gross, G., and Schindler, D.G. (1993). Specific activation and targeting of cytotoxic lymphocytes through chimeric single chains consisting of antibody-binding domains and the gamma or zeta subunits of the immunoglobulin and T-cell receptors. Proc Natl Acad Sci U S A 90(2), 720-724. doi: 10.1073/pnas.90.2.720.

Faitschuk, E., Nagy, V., Hombach, A.A., and Abken, H. (2016). A dual chain chimeric antigen receptor (CAR) in the native antibody format for targeting immune cells towards cancer cells without the need of an scFv. Gene Therapy 23(10), 718726. doi: 10.1038/gt.2016.48. 
Fedorov, V.D., Themeli, M., and Sadelain, M. (2013). PD-1- and CTLA-4-based inhibitory chimeric antigen receptors (iCARs) divert off-target immunotherapy responses. Sci Transl Med 5(215), 215ra172. doi: 10.1126/scitranslmed.3006597.

Ferlay, J., Ervik, M., Lam, F., Colombet, M., Mery, L., Piñeros, M., et al. (2018). Global Cancer Observatory: Cancer Today [Online]. Lyon, France: International Agency for Research on Cancer. Available: https://gco.iarc.fr/today [Accessed 01.11.2020 2020].

Finney, H.M., Lawson, A.D., Bebbington, C.R., and Weir, A.N. (1998). Chimeric receptors providing both primary and costimulatory signaling in $\mathrm{T}$ cells from a single gene product. $J$ Immunol 161(6), 2791-2797.

Freiberg, B.A., Kupfer, H., Maslanik, W., Delli, J., Kappler, J., Zaller, D.M., et al. (2002). Staging and resetting T cell activation in SMACs. Nature Immunology 3(10), 911-917. doi: 10.1038/ni836.

Fuhlbrigge, R.C., Kieffer, J.D., Armerding, D., and Kupper, T.S. (1997). Cutaneous lymphocyte antigen is a specialized form of PSGL-1 expressed on skin-homing T cells. Nature 389(6654), 978-981. doi: 10.1038/40166.

Gacerez, A.T., Arellano, B., and Sentman, C.L. (2016). How Chimeric Antigen Receptor Design Affects Adoptive T Cell Therapy. J Cell Physiol 231(12), 2590-2598. doi: 10.1002/jcp.25419.

Garcia, K.C., Degano, M., Stanfield, R.L., Brunmark, A., Jackson, M.R., Peterson, P.A., et al. (1996). An $\alpha \beta$ T Cell Receptor Structure at 2.5 $\AA$ and Its Orientation in the TCR-MHC Complex. Science 274(5285), 209-219. doi: 10.1126/science.274.5285.209.

Garfall, A.L., Dancy, E.K., Cohen, A.D., Hwang, W.-T., Fraietta, J.A., Davis, M.M., et al. (2019). T-cell phenotypes associated with effective CAR T-cell therapy in postinduction vs relapsed multiple myeloma. Blood advances 3(19), 2812-2815. doi: 10.1182/bloodadvances.2019000600.

Genkinger, J.M., Kitahara, C.M., Bernstein, L., Berrington de Gonzalez, A., Brotzman, M., Elena, J.W., et al. (2015). Central adiposity, obesity during early adulthood, and pancreatic cancer mortality in a pooled analysis of cohort studies. Annals of oncology : official journal of the European Society for Medical Oncology 26(11), 2257-2266. doi: 10.1093/annonc/mdv355.

Ghorashian, S., Kramer, A.M., Onuoha, S., Wright, G., Bartram, J., Richardson, R., et al. (2019). Enhanced CAR T cell expansion and prolonged persistence in pediatric patients with ALL treated with a low-affinity CD19 CAR. 25(9), 14081414. doi: 10.1038/s41591-019-0549-5.

Gillen, S., Schuster, T., Meyer zum Büschenfelde, C., Friess, H., and Kleeff, J. (2010). Preoperative/Neoadjuvant Therapy in Pancreatic Cancer: A Systematic Review and Meta-analysis of Response and Resection Percentages. PLOS Medicine 7(4), e1000267. doi: 10.1371/journal.pmed.1000267.

Golan, T., Hammel, P., Reni, M., Van Cutsem, E., Macarulla, T., Hall, M.J., et al. (2019). Maintenance Olaparib for Germline BRCA-Mutated Metastatic Pancreatic Cancer. $N$ Engl $J$ Med 381(4), 317-327. doi: 10.1056/NEJMoa1903387.

Gong, J., Chehrazi-Raffle, A., Reddi, S., and Salgia, R. (2018). Development of PD-1 and PD-L1 inhibitors as a form of cancer immunotherapy: a comprehensive review of registration trials and future considerations. 6(1), 8. doi: 10.1186/s40425-018-0316-z.

Griffin, J.F., Smalley, S.R., Jewell, W., Paradelo, J.C., Reymond, R.D., Hassanein, R.E., et al. (1990). Patterns of failure after curative resection of pancreatic carcinoma. 
Cancer 66(1), 56-61. doi: 10.1002/1097-0142(19900701)66:1<56::aidcncr2820660112>3.0.co;2-6.

Grippo, P.J., Nowlin, P.S., Demeure, M.J., Longnecker, D.S., and Sandgren, E.P. (2003). Preinvasive pancreatic neoplasia of ductal phenotype induced by acinar cell targeting of mutant Kras in transgenic mice. Cancer Res 63(9), 2016-2019.

Gross, G., Waks, T., and Eshhar, Z. (1989). Expression of immunoglobulin-T-cell receptor chimeric molecules as functional receptors with antibody-type specificity. Proc Natl Acad Sci U S A 86(24), 10024-10028. doi: 10.1073/pnas.86.24.10024.

Gudjonsson, B. (1995). Carcinoma of the pancreas: critical analysis of costs, results of resections, and the need for standardized reporting. $J$ Am Coll Surg 181(6), 483503.

Guedan, S., Chen, X., Madar, A., Carpenito, C., McGettigan, S.E., Frigault, M.J., et al. (2014). ICOS-based chimeric antigen receptors program bipolar TH17/TH1 cells. Blood 124(7), 1070-1080. doi: 10.1182/blood-2013-10-535245.

Guedan, S., Posey, A.D., Jr., Shaw, C., Wing, A., Da, T., Patel, P.R., et al. (2018). Enhancing CAR T cell persistence through ICOS and 4-1BB costimulation. JCI Insight 3(1). doi: 10.1172/jci.insight.96976.

Guest, R.D., Hawkins, R.E., Kirillova, N., Cheadle, E.J., Arnold, J., O'Neill, A., et al. (2005). The role of extracellular spacer regions in the optimal design of chimeric immune receptors: evaluation of four different scFvs and antigens. $J$ Immunother 28(3), 203-211. doi: 10.1097/01.cji.ooo0161397.96582.59.

Guo, Z.S. (2018). The 2018 Nobel Prize in medicine goes to cancer immunotherapy (editorial for BMC cancer). BMC Cancer 18(1), 1086. doi: 10.1186/s12885-0185020-3.

Hammarström, S. (1999). The carcinoembryonic antigen (CEA) family: structures, suggested functions and expression in normal and malignant tissues. Semin Cancer Biol 9(2), 67-81. doi: 10.1006/scbi.1998.0119.

Harris, L.J., Skaletsky, E., and McPherson, A. (1998). Crystallographic structure of an intact IgG1 monoclonal antibody11Edited by I. A. Wilson. Journal of Molecular Biology 275(5), 861-872. doi: https://doi.org/10.1006/jmbi.1997.1508.

Hartwig, W., Gluth, A., Hinz, U., Bergmann, F., Spronk, P.E., Hackert, T., et al. (2015). Total pancreatectomy for primary pancreatic neoplasms: renaissance of an unpopular operation. Ann Surg 261(3), 537-546. doi: 10.1097/sla.ooooooooooooo791.

Hartwig, W., Hackert, T., Hinz, U., Gluth, A., Bergmann, F., Strobel, O., et al. (2011). Pancreatic cancer surgery in the new millennium: better prediction of outcome. Ann Surg 254(2), 311-319. doi: 10.1097/SLA.obo13e31821fd334.

Hartwig, W., Werner, J., Jäger, D., Debus, J., and Büchler, M.W. (2013). Improvement of surgical results for pancreatic cancer. The Lancet Oncology 14(11), e476e485. doi: https://doi.org/10.1016/S1470-2045(13)70172-4.

Haso, W., Lee, D.W., Shah, N.N., Stetler-Stevenson, M., Yuan, C.M., Pastan, I.H., et al. (2013). Anti-CD22-chimeric antigen receptors targeting B-cell precursor acute lymphoblastic leukemia. Blood 121(7), 1165-1174. doi: 10.1182/blood-2012-06438002.

Haynes, N.M., Snook, M.B., Trapani, J.A., Cerruti, L., Jane, S.M., Smyth, M.J., et al. (2001). Redirecting mouse CTL against colon carcinoma: superior signaling efficacy of single-chain variable domain chimeras containing TCR-zeta vs Fc epsilon RI-gamma. $J$ Immunol 166(1), 182-187. doi: 10.4049/jimmunol.166.1.182. 
Hegde, M., DeRenzo, C.C., Zhang, H., Mata, M., Gerken, C., Shree, A., et al. (2017). Expansion of HER2-CAR T cells after lymphodepletion and clinical responses in patients with advanced sarcoma. Journal of Clinical Oncology 35(15_suppl), 10508-10508. doi: 10.1200/JCO.2017.35.15_suppl.10508.

Heinrich, S., Pestalozzi, B., Lesurtel, M., Berrevoet, F., Laurent, S., Delpero, J.-R., et al. (2011). Adjuvant gemcitabine versus NEOadjuvant gemcitabine/oxaliplatin plus adjuvant gemcitabine in resectable pancreatic cancer: a randomized multicenter phase III study (NEOPAC study). BMC cancer 11, 346-346. doi: 10.1186/1471-2407-11-346.

Henriksen, A., Dyhl-Polk, A., Chen, I., and Nielsen, D. (2019). Checkpoint inhibitors in pancreatic cancer. Cancer Treatment Reviews 78, 17-30. doi: 10.1016/j.ctrv.2019.06.005.

Henze, J., Tacke, F., Hardt, O., Alves, F., and Al Rawashdeh, W. (2020). Enhancing the Efficacy of CAR T Cells in the Tumor Microenvironment of Pancreatic Cancer. Cancers (Basel) 12(6). doi: 10.3390/cancers12061389.

Hodi, F.S., O'Day, S.J., McDermott, D.F., Weber, R.W., Sosman, J.A., Haanen, J.B., et al. (2010). Improved survival with ipilimumab in patients with metastatic melanoma. N Engl J Med 363(8), 711-723. doi: 10.1056/NEJMoa1003466.

Holter, S., Borgida, A., Dodd, A., Grant, R., Semotiuk, K., Hedley, D., et al. (2015). Germline BRCA Mutations in a Large Clinic-Based Cohort of Patients With Pancreatic Adenocarcinoma. $J$ Clin Oncol 33(28), 3124-3129. doi: 10.1200/jco.2014.59.7401.

Hombach, A., Hombach, A.A., and Abken, H. (2010). Adoptive immunotherapy with genetically engineered T cells: modification of the IgG1 Fc 'spacer' domain in the extracellular moiety of chimeric antigen receptors avoids 'off-target' activation and unintended initiation of an innate immune response. Gene Therapy 17(10), 1206-1213. doi: 10.1038/gt.2010.91.

Hombach, A., Sircar, R., Heuser, C., Tillmann, T., Diehl, V., Kruis, W., et al. (1998). Chimeric anti-TAG72 receptors with immunoglobulin constant Fc domains and gamma or zeta signalling chains. Int $J$ Mol Med 2(1), 99-103. doi: 10.3892/ijmm.2.1.99.

Hombach, A.A., Chmielewski, M., Rappl, G., and Abken, H. (2013). Adoptive immunotherapy with redirected $\mathrm{T}$ cells produces CCR7- cells that are trapped in the periphery and benefit from combined CD28-OX40 costimulation. Hum Gene Ther 24(3), 259-269. doi: 10.1089/hum.2012.247.

Hombach, A.A., Heiders, J., Foppe, M., Chmielewski, M., and Abken, H. (2012). OX4O costimulation by a chimeric antigen receptor abrogates CD28 and IL-2 induced IL-10 secretion by redirected CD4(+) T cells. Oncoimmunology 1(4), 458-466. doi: 10.4161/onci.19855.

Hosein, A.N., and Brekken, R.A. (2020). Pancreatic cancer stroma: an update on therapeutic targeting strategies. $17(8), 487-505$. doi: 10.1038/s41575-0200300-1.

Hoyos, V., Savoldo, B., Quintarelli, C., Mahendravada, A., Zhang, M., Vera, J., et al. (2010). Engineering CD19-specific T lymphocytes with interleukin-15 and a suicide gene to enhance their anti-lymphoma/leukemia effects and safety. Leukemia 24(6), 1160-1170. doi: 10.1038/leu.2010.75.

Hudecek, M., Lupo-Stanghellini, M.T., Kosasih, P.L., Sommermeyer, D., Jensen, M.C., Rader, C., et al. (2013). Receptor affinity and extracellular domain modifications affect tumor recognition by ROR1-specific chimeric antigen receptor T cells. Clin Cancer Res 19(12), 3153-3164. doi: 10.1158/1078-0432.ccr-13-0330. 
Hudecek, M., Sommermeyer, D., Kosasih, P.L., Silva-Benedict, A., Liu, L., Rader, C., et al. (2015). The nonsignaling extracellular spacer domain of chimeric antigen receptors is decisive for in vivo antitumor activity. Cancer Immunol Res 3(2), 125-135. doi: 10.1158/2326-6066.cir-14-0127.

Hudis, C.A. (2007). Trastuzumab - Mechanism of Action and Use in Clinical Practice. New England Journal of Medicine 357(1), 39-51. doi: 10.1056/NEJMra043186.

Irles, C., Symons, A., Michel, F., Bakker, T.R., van der Merwe, P.A., and Acuto, O. (2003). CD45 ectodomain controls interaction with GEMs and Lck activity for optimal TCR signaling. Nature Immunology 4(2), 189-197. doi: 10.1038/ni877.

Ishida, Y., Agata, Y., Shibahara, K., and Honjo, T. (1992). Induced expression of PD-1, a novel member of the immunoglobulin gene superfamily, upon programmed cell death. Embo j 11(11), 3887-3895.

James, S.E., Greenberg, P.D., Jensen, M.C., Lin, Y., Wang, J., Till, B.G., et al. (2008). Antigen sensitivity of CD22-specific chimeric TCR is modulated by target epitope distance from the cell membrane. J Immunol 180(10), 7028-7038. doi: 10.4049/jimmunol.180.10.7028.

Jiang, L., Wang, M., Lin, S., Jian, R., Li, X., Chan, J., et al. (2020). A Quantitative Proteome Map of the Human Body. Cell 183(1), 269-283.e219. doi: 10.1016/j.cell.2020.08.036.

Jonnalagadda, M., Mardiros, A., Urak, R., Wang, X., Hoffman, L.J., Bernanke, A., et al. (2015). Chimeric Antigen Receptors With Mutated IgG4 Fc Spacer Avoid Fc Receptor Binding and Improve T Cell Persistence and Antitumor Efficacy. Molecular Therapy 23(4), 757-768. doi: 10.1038/mt.2014.208.

June, C.H., and Sadelain, M. (2018). Chimeric Antigen Receptor Therapy. N Engl J Med 379(1), 64-73. doi: 10.1056/NEJMra1706169.

Kagoya, Y., Tanaka, S., and Guo, T. (2018). A novel chimeric antigen receptor containing a JAK-STAT signaling domain mediates superior antitumor effects. 24(3), 352-359. doi: 10.1038/nm.4478.

Kalser, M.H., and Ellenberg, S.S. (1985). Pancreatic cancer. Adjuvant combined radiation and chemotherapy following curative resection. Arch Surg 120(8), 899-903. doi: 10.1001/archsurg.1985.01390320023003.

Kanda, M., Matthaei, H., Wu, J., Hong, S.M., Yu, J., Borges, M., et al. (2012). Presence of somatic mutations in most early-stage pancreatic intraepithelial neoplasia. Gastroenterology 142(4), 730-733.e739. doi: 10.1053/j.gastro.2011.12.042.

Katari, U.L., Keirnan, J.M., Worth, A.C., Hodges, S.E., Leen, A.M., Fisher, W.E., et al. (2011). Engineered T cells for pancreatic cancer treatment. HPB 13(9), 643-650. doi: 10.1111/j.1477-2574.2011.00344.x.

Katz, M.H., Shi, Q., Ahmad, S.A., Herman, J.M., Marsh Rde, W., Collisson, E., et al. (2016). Preoperative Modified FOLFIRINOX Treatment Followed by Capecitabine-Based Chemoradiation for Borderline Resectable Pancreatic Cancer: Alliance for Clinical Trials in Oncology Trial A021101. JAMA Surg 151(8), e161137. doi: 10.1001/jamasurg.2016.1137.

Katz, S.C., Burga, R.A., McCormack, E., Wang, L.J., Mooring, W., Point, G.R., et al. (2015). Phase I Hepatic Immunotherapy for Metastases Study of Intra-Arterial Chimeric Antigen Receptor-Modified T-cell Therapy for CEA+ Liver Metastases. Clin Cancer Res 21(14), 3149-3159. doi: 10.1158/1078-0432.ccr-141421.

Kawalekar, O.U., RS, O.C., Fraietta, J.A., Guo, L., McGettigan, S.E., Posey, A.D., Jr., et al. (2016). Distinct Signaling of Coreceptors Regulates Specific Metabolism Pathways and Impacts Memory Development in CAR T Cells. Immunity 44(3), 712. doi: 10.1016/j.immuni.2016.02.023. 
Keller, L., Werner, S., and Pantel, K. (2019). Biology and clinical relevance of EpCAM. Cell Stress 3(6), 165-180. doi: 10.15698/cst2019.06.188.

Kim, M.-S., Pinto, S.M., Getnet, D., Nirujogi, R.S., Manda, S.S., Chaerkady, R., et al. (2014). A draft map of the human proteome. Nature 509(7502), 575-581. doi: 10.1038/nature13302.

Kleeff, J., Korc, M., Apte, M., La Vecchia, C., Johnson, C.D., Biankin, A.V., et al. (2016). Pancreatic cancer. Nature Reviews Disease Primers 2(1), 16022. doi: 10.1038/nrdp.2016.22.

Kloss, C.C., Condomines, M., Cartellieri, M., Bachmann, M., and Sadelain, M. (2013). Combinatorial antigen recognition with balanced signaling promotes selective tumor eradication by engineered T cells. Nat Biotechnol 31(1), 71-75. doi: $10.1038 / \mathrm{nbt} .2459$.

Koehler Leman, J., Ulmschneider, M.B., and Gray, J.J. (2015). Computational modeling of membrane proteins. Proteins 83(1), 1-24. doi: 10.1002/prot.24703.

Kolesnikov, N., Hastings, E., Keays, M., Melnichuk, O., Tang, Y.A., Williams, E., et al. (2014). ArrayExpress update-simplifying data submissions. Nucleic Acids Research 43(D1), D1113-D1116. doi: 10.1093/nar/gku1057.

Kopinke, D., Brailsford, M., Pan, F.C., Magnuson, M.A., Wright, C.V., and Murtaugh, L.C. (2012). Ongoing Notch signaling maintains phenotypic fidelity in the adult exocrine pancreas. Dev Biol 362(1), 57-64. doi: 10.1016/j.ydbio.2011.11.010.

Kopp, J.L., von Figura, G., Mayes, E., Liu, F.F., Dubois, C.L., Morris, J.P.t., et al. (2012). Identification of Sox9-dependent acinar-to-ductal reprogramming as the principal mechanism for initiation of pancreatic ductal adenocarcinoma. Cancer Cell 22(6), 737-750. doi: 10.1016/j.ccr.2012.10.025.

Krenciute, G., Krebs, S., Torres, D., Wu, M.F., Liu, H., Dotti, G., et al. (2016). Characterization and Functional Analysis of scFv-based Chimeric Antigen Receptors to Redirect T Cells to IL13Ra2-positive Glioma. Mol Ther 24(2), 354363. doi: 10.1038/mt.2015.199.

Künkele, A., Johnson, A.J., Rolczynski, L.S., Chang, C.A., Hoglund, V., Kelly-Spratt, K.S., et al. (2015). Functional Tuning of CARs Reveals Signaling Threshold above Which $\mathrm{CD} 8<\sup >+</ \sup >$ CTL Antitumor Potency Is Attenuated due to Cell Fas-FasL-Dependent AICD. Cancer Immunology Research 3(4), 368-379. doi: 10.1158/2326-6066.cir-14-0200.

Kuwana, Y., Asakura, Y., Utsunomiya, N., Nakanishi, M., Arata, Y., Itoh, S., et al. (1987). Expression of chimeric receptor composed of immunoglobulin-derived $\mathrm{V}$ regions and T-cell receptor-derived C regions. Biochem Biophys Res Commun 149(3), 960-968. doi: 10.1016/0006-291x(87)90502-x.

Lai, E., Puzzoni, M., Ziranu, P., Pretta, A., Impera, V., Mariani, S., et al. (2019). New therapeutic targets in pancreatic cancer. Cancer Treat Rev 81, 101926. doi: 10.1016/j.ctrv.2019.101926.

Lanitis, E., Poussin, M., Klattenhoff, A.W., Song, D., Sandaltzopoulos, R., June, C.H., et al. (2013). Chimeric antigen receptor $\mathrm{T}$ Cells with dissociated signaling domains exhibit focused antitumor activity with reduced potential for toxicity in vivo. Cancer Immunol Res 1(1), 43-53. doi: 10.1158/2326-6066.cir-13-00o8.

Larsson, S.C., and Wolk, A. (2012). Red and processed meat consumption and risk of pancreatic cancer: meta-analysis of prospective studies. $\mathrm{Br} J$ Cancer 106(3), 603-607. doi: 10.1038/bjc.2011.585.

Leach, D.R., Krummel, M.F., and Allison, J.P. (1996). Enhancement of antitumor immunity by CTLA-4 blockade. Science 271(5256), 1734-1736. doi: 10.1126/science.271.5256.1734. 
Leahy, D.J., Axel, R., and Hendrickson, W.A. (1992). Crystal structure of a soluble form of the human T cell coreceptor CD8 at 2.6 \&\#xc5; resolution. Cell 68(6), 11451162. doi: 10.1016/0092-8674(92)90085-Q.

Leen, A.M., Sukumaran, S., Watanabe, N., Mohammed, S., Keirnan, J., Yanagisawa, R., et al. (2014). Reversal of Tumor Immune Inhibition Using a Chimeric Cytokine Receptor. Molecular Therapy 22(6), 1211-1220. doi: 10.1038/mt.2014.47.

Lennernäs, B., Albertsson, P., Lennernäs, H., and Norrby, K. (2003). Chemotherapy and Antiangiogenesis. Acta Oncologica 42(4), 294-303. doi: 10.1080/02841860310001835.

Lenschow, D.J., Walunas, T.L., and Bluestone, J.A. (1996). CD28/B7 system of T cell costimulation. Annu Rev Immunol 14, 233-258. doi: 10.1146/annurev.immunol.14.1.233.

Lin, J.-R., Fallahi-Sichani, M., and Sorger, P.K. (2015). Highly multiplexed imaging of single cells using a high-throughput cyclic immunofluorescence method. Nature Communications 6(1), 8390. doi: 10.1038/ncomms9390.

Liu, Q., Sun, Z., and Chen, L. (2020). Memory T cells: strategies for optimizing tumor immunotherapy. Protein \& Cell 11(8), 549-564. doi: 10.1007/s13238-02000707-9.

Lynn, R.C., Feng, Y., Schutsky, K., Poussin, M., Kalota, A., Dimitrov, D.S., et al. (2016). High-affinity FR $\beta$-specific CAR T cells eradicate AML and normal myeloid lineage without HSC toxicity. Leukemia 30(6), 1355-1364. doi: 10.1038/leu.2016.35.

Macauley, M.S., Crocker, P.R., and Paulson, J.C. (2014). Siglec-mediated regulation of immune cell function in disease. Nat Rev Immunol 14(10), 653-666. doi: $10.1038 /$ nri3737.

Maher, J., Brentjens, R.J., Gunset, G., Rivière, I., and Sadelain, M. (2002). Human Tlymphocyte cytotoxicity and proliferation directed by a single chimeric TCR $\zeta$ /CD28 receptor. Nature Biotechnology 20(1), 70-75. doi: 10.1038/nbto102-70.

Makohon-Moore, A.P., Matsukuma, K., Zhang, M., Reiter, J.G., Gerold, J.M., Jiao, Y., et al. (2018). Precancerous neoplastic cells can move through the pancreatic ductal system. Nature 561(7722), 201-205. doi: 10.1038/s41586-018-0481-8.

Maliar, A., Servais, C., Waks, T., Chmielewski, M., Lavy, R., Altevogt, P., et al. (2012). Redirected T Cells That Target Pancreatic Adenocarcinoma Antigens Eliminate Tumors and Metastases in Mice. Gastroenterology 143(5), 1375-1384.e1375. doi: 10.1053/j.gastro.2012.07.017.

Martinelli, E., De Palma, R., Orditura, M., De Vita, F., and Ciardiello, F. (2009). Antiepidermal growth factor receptor monoclonal antibodies in cancer therapy. Clinical and experimental immunology 158(1), 1-9. doi: 10.1111/j.13652249.2009.03992.x.

Marx, V. (2019). A dream of single-cell proteomics. Nature Methods 16(9), 809-812. doi: 10.1038/s41592-019-0540-6.

Mata, M., Gerken, C., Nguyen, P., Krenciute, G., Spencer, D.M., and Gottschalk, S. (2017). Inducible Activation of MyD88 and CD40 in CAR T Cells Results in Controllable and Potent Antitumor Activity in Preclinical Solid Tumor Models. Cancer Discov 7(11), 1306-1319. doi: 10.1158/2159-8290.cd-17-0263.

Miliotou, A.N., and Papadopoulou, L.C. (2018). CAR T-cell Therapy: A New Era in Cancer Immunotherapy. Curr Pharm Biotechnol 19(1), 5-18. doi: 10.2174/1389201019666180418095526.

Mohammed, S., Sukumaran, S., Bajgain, P., Watanabe, N., Heslop, H.E., Rooney, C.M., et al. (2017). Improving Chimeric Antigen Receptor-Modified T Cell Function 
by Reversing the Immunosuppressive Tumor Microenvironment of Pancreatic Cancer. Molecular Therapy 25(1), 249-258. doi: 10.1016/j.ymthe.2016.10.016.

Monks, C.R.F., Freiberg, B.A., Kupfer, H., Sciaky, N., and Kupfer, A. (1998). Threedimensional segregation of supramolecular activation clusters in T cells. Nature 395(6697), 82-86. doi: 10.1038/25764.

Morgan, R.A., Yang, J.C., Kitano, M., Dudley, M.E., Laurencot, C.M., and Rosenberg, S.A. (2010). Case report of a serious adverse event following the administration of T cells transduced with a chimeric antigen receptor recognizing ERBB2. Mol Ther 18(4), 843-851. doi: 10.1038/mt.2010.24.

Morgenroth, A., Cartellieri, M., Schmitz, M., Günes, S., Weigle, B., Bachmann, M., et al. (2007). Targeting of tumor cells expressing the prostate stem cell antigen (PSCA) using genetically engineered T-cells. Prostate 67(10), 1121-1131. doi: 10.1002/pros.20608.

Moritz, D., and Groner, B. (1995). A spacer region between the single chain antibodyand the $\mathrm{CD}_{3}$ zeta-chain domain of chimeric $\mathrm{T}$ cell receptor components is required for efficient ligand binding and signaling activity. Gene Ther 2(8), 539546.

Morsut, L., Roybal, K.T., Xiong, X., Gordley, R.M., Coyle, S.M., Thomson, M., et al. (2016). Engineering Customized Cell Sensing and Response Behaviors Using Synthetic Notch Receptors. Cell 164(4), 780-791. doi: 10.1016/j.cell.2016.01.012.

Mount, C.W., Majzner, R.G., Sundaresh, S., Arnold, E.P., Kadapakkam, M., Haile, S., et al. (2018). Potent antitumor efficacy of anti-GD2 CAR T cells in H3-K27M+ diffuse midline gliomas. Nature Medicine 24(5), 572-579. doi: 10.1038/s41591018-0006-x.

Nemunaitis, J. (2005). Vaccines in cancer: GVAX, a GM-CSF gene vaccine. Expert Rev Vaccines 4(3), 259-274. doi: 10.1586/14760584.4.3.259.

Neoptolemos, J.P., Kleeff, J., Michl, P., Costello, E., Greenhalf, W., and Palmer, D.H. (2018). Therapeutic developments in pancreatic cancer: current and future perspectives. Nat Rev Gastroenterol Hepatol 15(6), 333-348. doi: 10.1038/s41575-018-0005-X.

Neoptolemos, J.P., Palmer, D.H., Ghaneh, P., Psarelli, E.E., Valle, J.W., Halloran, C.M., et al. (2017). Comparison of adjuvant gemcitabine and capecitabine with gemcitabine monotherapy in patients with resected pancreatic cancer (ESPAC4): a multicentre, open-label, randomised, phase 3 trial. The Lancet 389(10073), 1011-1024. doi: 10.1016/So140-6736(16)32409-6.

Neoptolemos, J.P., Stocken, D.D., Bassi, C., Ghaneh, P., Cunningham, D., Goldstein, D., et al. (2010). Adjuvant Chemotherapy With Fluorouracil Plus Folinic Acid vs Gemcitabine Following Pancreatic Cancer Resection: A Randomized Controlled Trial. JAMA 304(10), 1073-1081. doi: 10.1001/jama.2010.1275.

Neoptolemos, J.P., Stocken, D.D., Friess, H., Bassi, C., Dunn, J.A., Hickey, H., et al. (2004). A randomized trial of chemoradiotherapy and chemotherapy after resection of pancreatic cancer. $N$ Engl $J$ Med 350(12), 1200-1210. doi: 10.1056/NEJMoa032295.

Neuhaus, P., Riess, H., Post, S., Gellert, K., Ridwelski, K., Schramm, H., et al. (2008). CONKO-O01: Final results of the randomized, prospective, multicenter phase III trial of adjuvant chemotherapy with gemcitabine versus observation in patients with resected pancreatic cancer (PC). Journal of Clinical Oncology 26(15_suppl), LBA4504-LBA4504. doi: 10.1200/jco.2008.26.15_suppl.lba4504. 
Niederman, T.M.J., Ghogawala, Z., Carter, B.S., Tompkins, H.S., Russell, M.M., and Mulligan, R.C. (2002). Antitumor activity of cytotoxic T lymphocytes engineered to target vascular endothelial growth factor receptors. Proceedings of the National Academy of Sciences of the United States of America 99(10), 7009-7014. doi: 10.1073/pnas.092562399.

Oettle, H., Neuhaus, P., Hochhaus, A., Hartmann, J.T., Gellert, K., Ridwelski, K., et al. (2013). Adjuvant Chemotherapy With Gemcitabine and Long-term Outcomes Among Patients With Resected Pancreatic Cancer: The CONKO-001 Randomized Trial. JAMA 310(14), 1473-1481. doi: 10.1001/jama.2013.279201.

Oettle, H., Riess, H., Stieler, J.M., Heil, G., Schwaner, I., Seraphin, J., et al. (2014). Second-line oxaliplatin, folinic acid, and fluorouracil versus folinic acid and fluorouracil alone for gemcitabine-refractory pancreatic cancer: outcomes from the CONKO-003 trial. $J$ Clin Oncol 32(23), 2423-2429. doi: 10.1200/jco.2013.53.6995.

Ohmori, K., Fukui, F., Kiso, M., Imai, T., Yoshie, O., Hasegawa, H., et al. (2006). Identification of cutaneous lymphocyte-associated antigen as sialyl 6-sulfo Lewis X, a selectin ligand expressed on a subset of skin-homing helper memory T cells. Blood 107(8), 3197-3204. doi: 10.1182/blood-2005-05-2185.

Oken, M.M., Creech, R.H., Tormey, D.C., Horton, J., Davis, T.E., McFadden, E.T., et al. (1982). Toxicity and response criteria of the Eastern Cooperative Oncology Group. Am J Clin Oncol 5(6), 649-655.

Onnis, A., and Baldari, C.T. (2019). Orchestration of Immunological Synapse Assembly by Vesicular Trafficking. Frontiers in Cell and Developmental Biology 7(110). doi: 10.3389/fcell.2019.00110.

Orentas, R., Yang, J., Wen, X., Wei, J., Mackall, C., and Khan, J. (2012). Identification of Cell Surface Proteins as Potential Immunotherapy Targets in 12 Pediatric Cancers. Frontiers in Oncology 2(194). doi: 10.3389/fonc.2012.00194.

Orth, M., Metzger, P., Gerum, S., Mayerle, J., Schneider, G., Belka, C., et al. (2019). Pancreatic ductal adenocarcinoma: biological hallmarks, current status, and future perspectives of combined modality treatment approaches. Radiation Oncology 14(1), 141. doi: 10.1186/s13014-019-1345-6.

Parkin, D.M. (2011). 2. Tobacco-attributable cancer burden in the UK in 2010. British journal of cancer 105 Suppl 2(Suppl 2), S6-S13. doi: 10.1038/bjc.2011.475.

Patel, S.D., Moskalenko, M., Smith, D., Maske, B., Finer, M.H., and McArthur, J.G. (1999). Impact of chimeric immune receptor extracellular protein domains on T cell function. Gene Ther 6(3), 412-419. doi: 10.1038/sj.gt.3300831.

Pegram, H.J., Lee, J.C., Hayman, E.G., Imperato, G.H., Tedder, T.F., Sadelain, M., et al. (2012). Tumor-targeted T cells modified to secrete IL-12 eradicate systemic tumors without need for prior conditioning. Blood 119(18), 4133-4141. doi: 10.1182/blood-2011-12-400044.

Perna, F., Berman, S.H., Soni, R.K., Mansilla-Soto, J., Eyquem, J., Hamieh, M., et al. (2017). Integrating Proteomics and Transcriptomics for Systematic Combinatorial Chimeric Antigen Receptor Therapy of AML. Cancer Cell 32(4), 506-519.e505. doi: 10.1016/j.ccell.2017.09.004.

Pihlak, R., Valle, J.W., and McNamara, M.G. (2017). Germline mutations in pancreatic cancer and potential new therapeutic options. Oncotarget 8(42).

Posey, A.D., Jr., Schwab, R.D., Boesteanu, A.C., Steentoft, C., Mandel, U., Engels, B., et al. (2016). Engineered CAR T Cells Targeting the Cancer-Associated TnGlycoform of the Membrane Mucin MUC1 Control Adenocarcinoma. Immunity 44(6), 1444-1454. doi: 10.1016/j.immuni.2016.05.014. 
Rahib, L., Smith, B.D., Aizenberg, R., Rosenzweig, A.B., Fleshman, J.M., and Matrisian, L.M. (2014). Projecting cancer incidence and deaths to 2030: the unexpected burden of thyroid, liver, and pancreas cancers in the United States. Cancer Res 74(11), 2913-2921. doi: 10.1158/o0o8-5472.can-14-0155.

Reinhard, K., Rengstl, B., Oehm, P., Michel, K., Billmeier, A., Hayduk, N., et al. (2020). An RNA vaccine drives expansion and efficacy of claudin-CAR-T cells against solid tumors. Science 367(6476), 446-453. doi: 10.1126/science.aay5967.

Rohrmann, S., Linseisen, J., Nöthlings, U., Overvad, K., Egeberg, R., Tjønneland, A., et al. (2013). Meat and fish consumption and risk of pancreatic cancer: results from the European Prospective Investigation into Cancer and Nutrition. Int $J$ Cancer 132(3), 617-624. doi: 10.1002/ijc.27637.

Romeo, C., Amiot, M., and Seed, B. (1992). Sequence requirements for induction of cytolysis by the $\mathrm{T}$ cell antigen/Fc receptor zeta chain. Cell 68(5), 889-897. doi: 10.1016/0092-8674(92)90032-8.

Rovira, M., Scott, S.G., Liss, A.S., Jensen, J., Thayer, S.P., and Leach, S.D. (2010). Isolation and characterization of centroacinar/terminal ductal progenitor cells in adult mouse pancreas. Proc Natl Acad Sci US A 107(1), 75-80. doi: 10.1073/pnas.0912589107.

Roybal, K.T., Rupp, L.J., Morsut, L., Walker, W.J., McNally, K.A., Park, J.S., et al. (2016). Precision Tumor Recognition by T Cells With Combinatorial AntigenSensing Circuits. Cell 164(4), 770-779. doi: 10.1016/j.cell.2016.01.011.

Ryazantsev, S., Tischenko, V., Nguyen, C., Abramov, V., and Zav'yalov, V. (2013). Three-Dimensional Structure of the Human Myeloma IgG2. PLOS ONE 8(6), e64076. doi: 10.1371/journal.pone.0064076.

Schmidts, A., and Maus, M.V. (2018). Making CAR T Cells a Solid Option for Solid Tumors. Frontiers in Immunology 9(2593). doi: 10.3389/fimmu.2018.02593.

Schwanhäusser, B., Busse, D., Li, N., Dittmar, G., Schuchhardt, J., Wolf, J., et al. (2011). Global quantification of mammalian gene expression control. Nature 473(7347), 337-342. doi: 10.1038/nature10098.

Sethi, V., Giri, B., Saluja, A., and Dudeja, V. (2017). Insights into the Pathogenesis of Pancreatic Cystic Neoplasms. Dig Dis Sci 62(7), 1778-1786. doi: 10.1007/s10620-017-4603-1.

Seufferlein, T., and Ettrich, T.J. (2019). Treatment of pancreatic cancer-neoadjuvant treatment in resectable pancreatic cancer (PDAC). Translational gastroenterology and hepatology 4, 21-21. doi: 10.21037/tgh.2019.03.05.

Sezgin, E., Levental, I., Mayor, S., and Eggeling, C. (2017). The mystery of membrane organization: composition, regulation and roles of lipid rafts. Nature Reviews Molecular Cell Biology 18(6), 361-374. doi: 10.1038/nrm.2017.16.

Siegel, R., Naishadham, D., and Jemal, A. (2012). Cancer statistics, 2012. CA: A Cancer Journal for Clinicians 62(1), 10-29. doi: https://doi.org/10.3322/caac.20138.

Siegel, R.L., Miller, K.D., and Jemal, A. (2018). Cancer statistics, 2018. CA: A Cancer Journal for Clinicians 68(1), 7-30. doi: https://doi.org/10.3322/caac.21442.

Sohal, D.P., Mangu, P.B., Khorana, A.A., Shah, M.A., Philip, P.A., O'Reilly, E.M., et al. (2016). Metastatic Pancreatic Cancer: American Society of Clinical Oncology Clinical Practice Guideline. $J$ Clin Oncol 34(23), 2784-2796. doi: 10.1200/jco.2016.67.1412.

Sohal, D.P.S., Kennedy, E.B., Cinar, P., Conroy, T., Copur, M.S., Crane, C.H., et al. (2020). Metastatic Pancreatic Cancer: ASCO Guideline Update. J Clin Oncol, Jc02001364. doi: 10.1200/jco.20.01364. 
Song, D.G., and Powell, D.J. (2012). Pro-survival signaling via CD27 costimulation drives effective CAR T-cell therapy. Oncoimmunology 1(4), 547-549. doi: 10.4161/onci.19458.

Strunk, D., Egger, C., Leitner, G., Hanau, D., and Stingl, G. (1997). A skin homing molecule defines the langerhans cell progenitor in human peripheral blood. $J$ Exp Med 185(6), 1131-1136. doi: 10.1084/jem.185.6.1131.

Szöőr, Á., Tóth, G., Zsebik, B., Szabó, V., Eshhar, Z., Abken, H., et al. (2020). Trastuzumab derived HER2-specific CARs for the treatment of trastuzumabresistant breast cancer: CAR T cells penetrate and eradicate tumors that are not accessible to antibodies. Cancer Letters 484, 1-8. doi: https://doi.org/10.1016/j.canlet.2020.04.008.

Tachezy, M., Gebauer, F., Petersen, C., Arnold, D., Trepel, M., Wegscheider, K., et al. (2014). Sequential neoadjuvant chemoradiotherapy (CRT) followed by curative surgery vs. primary surgery alone for resectable, non-metastasized pancreatic adenocarcinoma: NEOPA- a randomized multicenter phase III study (NCTo1900327, DRKSooo03893, ISRCTN82191749). BMC cancer 14, 411-411. doi: 10.1186/1471-2407-14-411.

The UniProt Consortium (2019). UniProt: a worldwide hub of protein knowledge. Nucleic Acids Research 47(D1), D506-D515. doi: 10.1093/nar/gky1049.

Thistlethwaite, F.C., Gilham, D.E., Guest, R.D., Rothwell, D.G., Pillai, M., Burt, D.J., et al. (2017). The clinical efficacy of first-generation carcinoembryonic antigen (CEACAM5)-specific CAR T cells is limited by poor persistence and transient pre-conditioning-dependent respiratory toxicity. Cancer Immunol Immunother 66(11), 1425-1436. doi: 10.1007/s00262-017-2034-7.

Timp, W., and Timp, G. (2020). Beyond mass spectrometry, the next step in proteomics. Science Advances 6(2), eaax8978. doi: 10.1126/sciadv.aax8978.

Tokarew, N., Ogonek, J., Endres, S., von Bergwelt-Baildon, M., and Kobold, S. (2019). Teaching an old dog new tricks: next-generation CAR T cells. $\mathrm{Br} J$ Cancer 120(1), 26-37. doi: 10.1038/s41416-018-0325-1.

Tsuchiyama, J., Yoshino, T., Toba, K., Harada, N., Nishiuchi, R., Akagi, T., et al. (2002). Induction and characterization of cutaneous lymphocyte antigen on natural killer cells. $\mathrm{Br} \quad J$ Haematol 118(2), 654-662. doi: 10.1046/j.13652141.2002.03608.x.

Tu, L., Delahunty, M.D., Ding, H., Luscinskas, F.W., and Tedder, T.F. (1999). The cutaneous lymphocyte antigen is an essential component of the L-selectin ligand induced on human vascular endothelial cells. The Journal of experimental medicine 189(2), 241-252. doi: 10.1084/jem.189.2.241.

Turati, F., Edefonti, V., Bosetti, C., Ferraroni, M., Malvezzi, M., Franceschi, S., et al. (2013). Family history of cancer and the risk of cancer: a network of case-control studies. Ann Oncol 24(10), 2651-2656. doi: 10.1093/annonc/mdt280.

Uesaka, K., Boku, N., Fukutomi, A., Okamura, Y., Konishi, M., Matsumoto, I., et al. (2016). Adjuvant chemotherapy of S-1 versus gemcitabine for resected pancreatic cancer: a phase 3, open-label, randomised, non-inferiority trial (JASPAC 01). The Lancet 388(10041), 248-257. doi: 10.1016/So1406736(16)30583-9.

Uhlén, M., Fagerberg, L., Hallström, B.M., Lindskog, C., Oksvold, P., Mardinoglu, A., et al. (2015). Tissue-based map of the human proteome. Science 347(6220), 1260419. doi: 10.1126/science.1260419.

Verschoor, C.P., Lelic, A., Bramson, J.L., and Bowdish, D.M.E. (2015). An Introduction to Automated Flow Cytometry Gating Tools and Their Implementation. Frontiers in Immunology 6(380). doi: 10.3389/fimmu.2015.00380. 
Versteijne, E., van Eijck, C.H.J., Punt, C.J.A., Suker, M., Zwinderman, A.H., Dohmen, M.A.C., et al. (2016). Preoperative radiochemotherapy versus immediate surgery for resectable and borderline resectable pancreatic cancer (PREOPANC trial): study protocol for a multicentre randomized controlled trial. Trials 17(1), 127-127. doi: 10.1186/s13063-016-1262-z.

Von Hoff, D.D., Ramanathan, R.K., Borad, M.J., Laheru, D.A., Smith, L.S., Wood, T.E., et al. (2011). Gemcitabine plus nab-paclitaxel is an active regimen in patients with advanced pancreatic cancer: a phase I/II trial. J Clin Oncol 29(34), 45484554. doi: 10.1200/jco.2011.36.5742.

Waddell, N., Pajic, M., Patch, A.M., Chang, D.K., Kassahn, K.S., Bailey, P., et al. (2015). Whole genomes redefine the mutational landscape of pancreatic cancer. Nature 518(7540), 495-501. doi: 10.1038/nature14169.

Wang-Gillam, A., Li, C.P., Bodoky, G., Dean, A., Shan, Y.S., Jameson, G., et al. (2016). Nanoliposomal irinotecan with fluorouracil and folinic acid in metastatic pancreatic cancer after previous gemcitabine-based therapy (NAPOLI-1): a global, randomised, open-label, phase 3 trial. Lancet 387(10018), 545-557. doi: 10.1016/s0140-6736(15)oo986-1.

Wang, E., Wang, L.C., Tsai, C.Y., Bhoj, V., Gershenson, Z., Moon, E., et al. (2015). Generation of Potent T-cell Immunotherapy for Cancer Using DAP12-Based, Multichain, Chimeric Immunoreceptors. Cancer Immunol Res 3(7), 815-826. doi: 10.1158/2326-6066.cir-15-0054.

Wang, J., Jensen, M., Lin, Y., Sui, X., Chen, E., Lindgren, C.G., et al. (2007). Optimizing adoptive polyclonal $\mathrm{T}$ cell immunotherapy of lymphomas, using a chimeric $\mathrm{T}$ cell receptor possessing CD28 and CD137 costimulatory domains. Hum Gene Ther 18(8), 712-725. doi: 10.1089/hum.2007.028.

Wang, L., Xie, D., and Wei, D. (2019). Pancreatic Acinar-to-Ductal Metaplasia and Pancreatic Cancer. Methods Mol Biol 1882, 299-308. doi: 10.1007/978-1-49398879-2_26.

Wang, Y., Chen, M., Wu, Z., Tong, C., Dai, H., Guo, Y., et al. (2018). CD133-directed CAR T cells for advanced metastasis malignancies: A phase I trial. Oncoimmunology 7(7), e1440169. doi: 10.1080/2162402x.2018.1440169.

Watanabe, K., Kuramitsu, S., Posey, A.D., and June, C.H. (2018). Expanding the Therapeutic Window for CAR T Cell Therapy in Solid Tumors: The Knowns and Unknowns of CAR T Cell Biology. Frontiers in Immunology 9(2486). doi: 10.3389/fimmu.2018.02486.

Watanabe, K., Terakura, S., Uchiyama, S., Martens, A.C., Meerten, T.v., Kiyoi, H., et al. (2014). Excessively High-Affinity Single-Chain Fragment Variable Region in a Chimeric Antigen Receptor Can Counteract T-Cell Proliferation. Blood 124(21), 4799-4799. doi: 10.1182/blood.V124.21.4799.4799.

Watanabe, N., Bajgain, P., Sukumaran, S., Ansari, S., Heslop, H.E., Rooney, C.M., et al. (2016). Fine-tuning the CAR spacer improves T-cell potency. Oncoimmunology 5(12), e1253656-e1253656. doi: 10.1080/2162402X.2016.1253656.

Weijtens, M.E., Willemsen, R.A., van Krimpen, B.A., and Bolhuis, R.L. (1998). Chimeric scFv/gamma receptor-mediated T-cell lysis of tumor cells is coregulated by adhesion and accessory molecules. Int $J$ Cancer 77(2), 181-187. doi: 10.1002/(sici)1097-0215(19980717)77:2<181::aid-ijc2>3.0.co;2-m.

Werner, J., Combs, S.E., Springfeld, C., Hartwig, W., Hackert, T., and Büchler, M.W. (2013). Advanced-stage pancreatic cancer: therapy options. Nat Rev Clin Oncol 10(6), 323-333. doi: 10.1038/nrclinonc.2013.66. 
Whiteman, D.C., Webb, P.M., Green, A.C., Neale, R.E., Fritschi, L., Bain, C.J., et al. (2015). Cancers in Australia in 2010 attributable to modifiable factors: introduction and overview. Australian and New Zealand journal of public health 39(5), 403-407. doi: 10.1111/1753-6405.12468.

Wilhelm, M., Schlegl, J., Hahne, H., Gholami, A.M., Lieberenz, M., Savitski, M.M., et al. (2014). Mass-spectrometry-based draft of the human proteome. Nature 509(7502), 582-587. doi: 10.1038/nature13319.

Wilkie, S., Picco, G., Foster, J., Davies, D.M., Julien, S., Cooper, L., et al. (2008). Retargeting of Human T Cells to Tumor-Associated MUC1: The Evolution of a Chimeric Antigen Receptor. The Journal of Immunology 180(7), 4901. doi: 10.4049/jimmunol.180.7.4901.

Wilkie, S., van Schalkwyk, M.C., Hobbs, S., Davies, D.M., van der Stegen, S.J., Pereira, A.C., et al. (2012). Dual targeting of ErbB2 and MUC1 in breast cancer using chimeric antigen receptors engineered to provide complementary signaling. $J$ Clin Immunol 32(5), 1059-1070. doi: 10.1007/s10875-012-9689-9.

Witkiewicz, A.K., McMillan, E.A., Balaji, U., Baek, G., Lin, W.C., Mansour, J., et al. (2015). Whole-exome sequencing of pancreatic cancer defines genetic diversity and therapeutic targets. Nat Commun 6, 6744. doi: 10.1038/ncomms7744.

Wolak, D.J., Pizzo, M.E., and Thorne, R.G. (2015). Probing the extracellular diffusion of antibodies in brain using in vivo integrative optical imaging and ex vivo fluorescence imaging. Journal of Controlled Release 197, 78-86. doi: https://doi.org/10.1016/j.jconrel.2014.10.034.

Wortmann, A., He, Y., Deryugina, E.I., Quigley, J.P., and Hooper, J.D. (2009). The cell surface glycoprotein CDCP1 in cancer--insights, opportunities, and challenges. IUBMB Life 61(7), 723-730. doi: 10.1002/iub.198.

Wu, L., Wei, Q., Brzostek, J., and Gascoigne, N.R.J. (2020). Signaling from T cell receptors (TCRs) and chimeric antigen receptors (CARs) on T cells. Cellular \& Molecular Immunology 17(6), 600-612. doi: 10.1038/s41423-020-0470-3.

Yamamoto, K., Venida, A., Yano, J., Biancur, D.E., Kakiuchi, M., Gupta, S., et al. (2020). Autophagy promotes immune evasion of pancreatic cancer by degrading MHC-I. 581(7806), 100-105. doi: 10.1038/s41586-020-2229-5.

Yoshino, T., Okano, M., Chen, H.L., Tsuchiyama, J., Kondo, E., Nishiuchi, R., et al. (1999). Cutaneous lymphocyte antigen is expressed on memory/effector B cells in the peripheral blood and monocytoid B cells in the lymphoid tissues. Cell Immunol 197(1), 39-45. doi: 10.1006/cimm.1999.1552.

Zhang, T., Lemoi, B.A., and Sentman, C.L. (2005). Chimeric NK-receptor-bearing T cells mediate antitumor immunotherapy. Blood 106(5), 1544-1551. doi: 10.1182/blood-2004-11-4365.

Zheng, P.-P., Kros, J.M., and Li, J. (2018). Approved CAR T cell therapies: ice bucket challenges on glaring safety risks and long-term impacts. Drug Discovery Today 23(6), 1175-1182. doi: https://doi.org/10.1016/j.drudis.2018.02.012. 


\section{Acknowledgements}

I want to express my deepest gratitude to Dr. Olaf Hardt, for giving me a chance, continuous support, supervision and motivation. He not only guided me through this thesis, but gave me also freedom and opportunity to grow as a scientist, character and be visible at various occasions.

I want to thank Prof. Dr. Frauke Alves for enabling me to work on this collaborative project. She supervised and supported me over the last years and was ever helpful and available. Our discussions helped changing my perspective on the problems at hand and thanks to you gathered and maintained my interest in the world of imaging.

Furthermore, I want to thank my TAC members Prof. Dr. Ralf Dressel and Prof. Dr. Hubertus Jarry. I always left our meetings with a very good feeling and stronger self-confidence. I appreciate very much the scientific but also mental support offered by them and supportive attitude. A good feeling can often be more motivating than a good result.

In addition, I want to convey my gratitude to Prof. Dr. Heidi Hahn, Prof. Dr. Lutz Walter and Prof. Dr. Luis Pardo for their interest in my work and their participation in my examination board. Schedules are ever tight and I appreciate this very much.

Many thanks are also owed to Laura Nadine Preiß. You led me through Miltenyi on my initial days and supported me throughout this project as colleague and friend. I am happy that we could work so great together.

Moreover, I want to thank Dr. Stefan Tomiuk for his great efforts on the field of bioinformatics that brought this project forward. You were always available and found always time for me, although your schedule was always crowded.

I want to thank Dr. Wa'el Al Rawashdeh for opening up the world of "in vivo" and imaging for me. You supported me as friend and mentor and this is much appreciated.

Many thanks to fabulous Cathrin Linnartz and Janina Brauner. You helped me so much during in vivo times and in hard times stood up for me without asking. Thanks for having my back.

I want to thank Anne Franke, Lena Wilnow, Benjamin Theiss and David Agorku for continued support and jumping in the breach when I was impeded. 
Many thanks also to all my colleagues in Göttingen, but especially Dr. Jeannine MissbachGüntner and Dr. Diana Pinkert-Leetsch. I enjoyed our collaboration throughout the years and I am grateful for your supported and input.

I want to express my gratitude towards all my co-authors of the manuscripts. You made it possible to have this manuscript based thesis and I am very thankful for this.

Many thanks are owed to Janina Henze, who was a formidable friend and shared first author through this project.

I want to thank Jonathan Druge, Jona Drushku, Aparajita Singh, Joanna Nowacka, Alba-Maria Albert I Robledo and Gene Swinerd, who are great colleagues and even better friends. You eased the way for me and were ever supportive. I hope we can have many more of our "Telcos" in the future.

Finally, I want to thank my parents and wonderful wife. Your unconditional support in every imaginable way all throughout my life made me feel strong enough to reach my goals, which is why this thesis is dedicated to you!

It has been an interesting journey to draft this thesis. Famous "Reviewer 2" gave me a hard time ... twice. A dormouse cut lab electricity on the day of tumor delivery, lab pipes broke during the final in vivo day, cyclic IF runs planned over the weekend ended right after you left the lab on Friday $9 \mathrm{pm}$. Quants reserved for weeks were clogged by colleagues 5 minutes before sample measurement and unforgettable the IT issue four weeks before thesis submission, preventing me from accessing any data from the last three years. Many more unfortunate events took place and that this thesis is still written would not have been possible without the support of the wonderful people around me to whom I owe my gratitude. 


\section{Curriculum Vitae}

\section{Daniel Schäfer}

Curriculum Vitae

Personal information

Place and date of birth:

18/06/1992, Freudenberg

Education

12/2017 - present Molecular Medicine PhD candidate

University Medical Center Göttingen

$09 / 2015-02 / 2018$

Biological Sciences M.Sc. with distinction (Grade 1.1)

University of Cologne/University of Manchester

10/2012 - 09/2015 Biological Sciences B.Sc. (Grade 2.1)

University of Cologne/University of Antwerp

$07 / 2002-06 / 2011$

General Higher Education Entrance Qualification (Grade 1.5)

Scholarships

01/2016-02/2018 Scholarship of the Friedrich-Ebert-Foundation

09/2015 - 03/2016 ERASMUS Scholarship

University of Manchester

02/2014-04/2014 CEPLAS Research Fellowship

University of Cologne

Publications

Schäfer, D., Henze, J., Pfeifer, R., Schleicher, A., Brauner, J., Mockel-Tenbrinck, N., et al. (2020). A Novel Siglec-4 Derived Spacer Improves the Functionality of CAR T Cells Against MembraneProximal Epitopes. Frontiers in Immunology 11(1704). doi: 10.3389/fimmu.2020.01704. 